\title{
Normal and counter Evershed flows in the penumbra of sunspots: HINODE observations and MHD simulations
}

\author{
Dissertation \\ zur Erlangung des mathematisch-naturwissenschaftlichen Doktorgrades \\ "Doctor rerum naturalium" \\ der Georg-August-Universität Göttingen \\ im Promotionsprogramm PROPHYS \\ der Georg-August University School of Science (GAUSS)
}

vorgelegt von

Azaymi Litzi Siu Tapia

aus Escuintla, Mexiko

Göttingen, 2017 
Betreuungsausschuss

Prof. Dr. Sami K. Solanki

Max-Planck-Institut für Sonnensystemforschung, Göttingen, Germany

Dr. Andreas Lagg

Max-Planck-Institut für Sonnensystemforschung, Göttingen, Germany

Prof. Dr. Ansgar Reiners

Georg-August-Universität Göttingen, Göttingen, Germany

Mitglieder der Prüfungskommision

Referent: Prof. Dr. Sami K. Solanki

Max-Planck-Institut für Sonnensystemforschung, Göttingen, Germany

Korreferent: Prof. Dr. Wolfram Kollatschny

Georg-August-Universität Göttingen, Göttingen, Germany

2. Korreferent: Dr. Jorrit Leenaarts

Institute for Solar Physics, Department of Astronomy, Stockholm University, Stockholm, Sweden

Weitere Mitglieder der Prüfungskommission:

Dr. Maarit Käpylä

Max-Planck-Institut für Sonnensystemforschung, Göttingen, Germany

Prof. Dr. Ansgar Reiners

Georg-August-Universität Göttingen, Göttingen, Germany

Prof. Dr. Hardi Peter

Max-Planck-Institut für Sonnensystemforschung, Göttingen, Germany

Prof. Dr. Stefan Dreizler

Georg-August-Universität Göttingen, Göttingen, Germany

Tag der mündlichen Prüfung: 29.01.2018 


\section{Bibliografische Information der Deutschen Nationalbibliothek}

Die Deutsche Nationalbibliothek verzeichnet diese Publikation in der Deutschen Nationalbibliografie; detaillierte bibliografische Daten sind im Internet über http: //dnb . d-nb . de abrufbar.

ISBN 978-3-944072-59-3

uni-edition $\mathrm{GmbH} 2018$

http://www.uni-edition.de

(c) Azaymi Litzi Siu Tapia

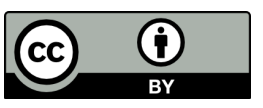

This work is distributed under a

Creative Commons Attribution 3.0 License

Printed in Germany 



\section{Contents}

$\begin{array}{ll}\text { Zusammenfassung } & 13\end{array}$

$\begin{array}{ll}\text { Summary } & 15\end{array}$

1 General Introduction 17

1.1 The Sun . . . . . . . . . . . . . . . . . . . . . . . . . . . . . 18

1.1.1 Internal structure . . . . . . . . . . . . . . . 18

1.1.2 Solar atmosphere . . . . . . . . . . . . . . 20

1.2 Solar lower atmosphere magnetism . . . . . . . . . . . . . . . . 22

1.3 Photospheric magnetic field measurements . . . . . . . . . . . . . 25

1.3.1 Doppler and Zeeman effect . . . . . . . . . . . . . . 25

1.3.2 Solar polarimetry . . . . . . . . . . . 30

1.3.3 Hinode spectropolarimeter . . . . . . . . . . . . . 36

1.3.4 Radiative transfer . . . . . . . . . . . . . . . . . . . . . . . . . . . . .

1.3.5 Inversions . . . . . . . . . . . . . . . . 40

1.3.5.1 The Milne-Eddington approximation . . . . . . . . 41

1.3.5.2 SPINOR inversions . . . . . . . . . . . 42

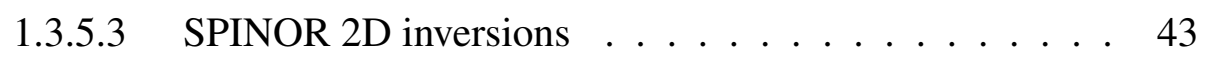

1.4 Magnetohydrodynamical simulations . . . . . . . . . . . . . . 43

1.4.1 The MURaM code . . . . . . . . . . . . . . . . . . 44

2 Sunspots: Review $\quad 47$

2.1 Sunspot formation and decay . . . . . . . . . . . . . . . . . . . . . 47

2.2 General properties of sunspots . . . . . . . . . . . . . . . . . . 49

2.3 The umbra . . . . . . . . . . . . . . . . . 52

2.4 On the existence of a penumbra . . . . . . . . . . 53

2.5 Penumbral fine structure $\ldots \ldots \ldots \ldots 5$

2.6 The Evershed flow . . . . . . . . . . . . . . . . . . . . 58

2.6.1 Some penumbral models . . . . . . . . . . . . . 59

2.6.2 Driving forces of the Evershed flow . . . . . . . . . . . . . . 62

2.7 Counter-Evershed flows $\ldots \ldots \ldots 66$

3 Normal and counter Evershed flows in the photospheric penumbra of a sunspot: $\begin{array}{ll}\text { SPINOR 2D inversions of Hinode-SOT/ SP observations } & \mathbf{7 3}\end{array}$

3.1 Introduction . . . . . . . . . . . . . . . . . . . . 74

3.2 Observational data and analysis techniques . . . . . . . . . . 76 
3.2 .1 Observations ..................... 76

3.2 .2 Inversions . . . . . . . . . . . . . . 76

3.3 Results . . . . . . . . . . . . . . . . . . 79

3.3.1 Filament selection . . . . . . . . . . . . . . 84

3.3.2 Qualitative picture of filaments . . . . . . . . . . . . . 89

3.3.2.1 Center-side and limb-side NEF filaments . . . . . . . 89

3.3.2.2 CEF filaments . . . . . . . . . . . . . . 91

3.3.2.3 Comparison between NEF and CEF filaments . . . . 93

3.4 Discussion . . . . . . . . . . . . . . . . . . . . . . . . 94

3.5 Conclusion . . . . . . . . . . . . . . . . . . 98

4 Normal and counter-Evershed flows in sunspot MHD simulations ${ }^{1} \quad 101$

4.1 Introduction . . . . . . . . . . . . . . . . . . . 101

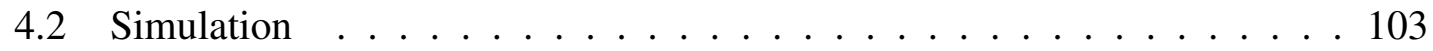

4.3 Results . . . . . . . . . . . . . . . . . . 105

4.3.1 Filamentary structure of the penumbra . . . . . . . . . 105

4.3.2 Driving forces of the penumbral flows . . . . . . . . . . . . 108

4.3.3 Temporal evolution of the CEF . . . . . . . . . . . 114

4.4 Induction equation . . . . . . . . . . . . . . . . 118

4.5 Field-line connectivity . . . . . . . . . . . . . . . . 121

4.6 Discussion and conclusion . . . . . . . . . . . . . . 123

5 Unusually large penumbral field strengths $\quad 129$

5.1 Introduction . . . . . . . . . . . . . . . . . . . . . . . 129

5.2 Zeeman splitting and center-of-gravity methods . . . . . . . . . . . 131

5.3 Inversions . . . . . . . . . . . . . . . . 136

5.3.1 Height-dependent inversions . . . . . . . . . . . . . . . 137

5.3.2 Height-independent inversions . . . . . . . . . . . . . . . 138

5.3 .3 Results . . . . . . . . . . . . . . . . . 138

5.4 Discussion . . . . . . . . . . . . . . . . . . . . 140

6 Conclusions and Outlook 147

6.1 Brief summary and conclusions . . . . . . . . . . . . . . 147

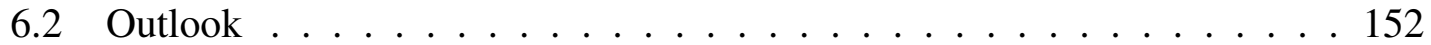

$\begin{array}{ll}\text { Bibliography } & 157\end{array}$

$\begin{array}{lr}\text { Publications } & 179\end{array}$

$\begin{array}{ll}\text { Acknowledgements } & 181\end{array}$

$\begin{array}{ll}\text { Curriculum vitae } & 183\end{array}$ 


\section{List of Figures}

1.1 Sketch of the internal structure of the Sun . . . . . . . . . . . . . . 19

1.2 Composite image of the solar atmosphere . . . . . . . . . . . . . 20

1.3 Temperature and density as functions of height in the solar atmosphere . . 21

1.4 The solar spectrum . . . . . . . . . . . . . . . . 22

1.5 Different regimes of solar magneto-convection in a G-band image taken from the Vacuum Tower Telescope (VTT) . . . . . . . . . . . . . . 23

1.6 Normal Zeeman effect . . . . . . . . . . . . . . . . . . . . . . 26

1.7 Anomalous Zeeman effect . . . . . . . . . . . . . . . . . 27

1.8 Polarization of the different Zeeman components in an emission line . . . 27

1.9 Possible states of polarization of a totally polarized electromagnetic wave 32

1.10 Linear retarder and linear analyzer-polarizer . . . . . . . . . . . . . 33

1.11 The influence of the strength and the orientation of the magnetic field on the four Stokes profiles of a spectral line . . . . . . . . . . . 35

2.1 Sketch of an emerging flux tube producing two pairs of spots with opposite field polarities . . . . . . . . . . . . . . . . . 48

2.2 Maps of physical parameters in a sunspot (AR NOAA 10933) observed near the solar disk center by the SOT/SP instrument on board Hinode . . 49

2.3 The Wilson depression at every point in an observed sunspot . . . . . . . 51

2.4 Continuum image of a sunspot taken at the Dunn Solar Tower at the National Solar Observatory . . . . . . . . . . . . . . . . 55

2.5 Classification of penumbral filaments according to their location within the penumbra . . . . . . . . . . . . . . . 56

2.6 Example of three bright penumbral filaments of different sizes and with dark central lanes observed near the umbra with the Swedish Solar Telescope $(\mathrm{SST}) \ldots \ldots \ldots \ldots$. . . . . . . . . . . . . . 56

2.7 Sketch of the interlocking-comb configuration of sunspot penumbrae . . . 57

2.8 The Evershed effect observed in AR NOAA 10933 as it moves away from the solar disk center . . . . . . . . . . . . . . . . 58

2.9 Two different penumbral models: The embedded flux-tube model and the field-free gap model . . . . . . . . . . . . . . . . . . . 60 60

2.10 Sketch of a siphon flow in a magnetic flux tube . . . . . . . . . 61

2.11 Sketches illustrating the radial convection in a penumbral filament and its lateral convective pattern . . . . . . . . . . . . 62

2.12 Variation of various physical parameters along the central axes of three 'standard filaments' . . . . . . . . . . . . . . 63 
2.13 The filamentary penumbra in a 3D MHD simulation of a sunspot . . . . . 64

2.14 CEFs observed with the ASP in the disk-center side of the $\delta$-sunspot penumbra in NOAA AR $7205 \ldots \ldots \ldots \ldots$

2.15 CEFs observed with Hinode SOT/SP on September 2011 along two unusual filaments that penetrate into the umbra of the sunspot in AR 11302 . 69

2.16 Two schematic scenarios proposed by Kleint and Sainz Dalda (2013) to explain the topology of unusual filaments observed carrying CEFs . . . . 70

2.17 CEFs observed with Hinode SOT/SP in the leading sunspot of NOAA AR 11271

3.1 Stokes maps observed by the Hinode SOT/SP in the main sunspot of the NOAA AR 10930 on December 08, 2006 . . . . . . . . . . . . 78

3.2 Observed Stokes profiles in NOAA AR 10930 and their SPINOR 2D bestfits . . . . . . . . . . . . . . . . . 79

3.3 SPINOR 2D inverted maps at three photospheric layers . . . . . . . . 81

3.4 Field inclination and field azimuth from SPINOR 2D inversions at three photospheric layers . . . . . . . . . . . . . . . . . 82

3.5 Continuum intensity maps of the center-side penumbra of main sunspot in NOAA AR $10930 \ldots \ldots$. . . . . . . . . . . . . . 83

3.6 Three regions of interest in the penumbra of NOAA AR 10930 . . . . . 84

3.7 Individual filaments carrying a NEF and a CEF in their de-stretched and scaled form . . . . . . . . . . . . . . . . 86

3.8 Transversal continuum intensity profiles of the average filaments . . . . . 87

3.9 Average filaments from the center-side NEF, limb-side NEF, and CEF region 88

3.10 Thermal, magnetic and velocity profiles along the central axes of average filaments at $\log (\tau)=0 \ldots \ldots \ldots$. . . . . . . . . . . 91

3.11 Variation of some physical parameters along the transversal cuts made across the filaments' heads and tails . . . . . . . . . . . . . . 92

3.12 Possible magnetic field configuration of the three average filaments from different sectors of the penumbra . . . . . . . . . . . . . . 93

3.13 Hinode G-band images showing the evolution of the anomalous penumbra in AR $10930 \ldots \ldots$. . . . . . . . . . . . . . . . . . . 95

4.1 Intensity image of a MURaM sunspot simulation that displays CEFs in some penumbral sectors . . . . . . . . . . . . . . . 103

4.2 Fine structure of the simulated sunspot at different optical depth levels . . 104

4.3 Vertical cross section through the central axes of two filaments in the simulation . . . . . . . . . . . . . . . . . . . 107

4.4 Radial flow velocity and radial energy conversion terms as functions of radius and height in the simulated penumbra . . . . . . . . . . . . . 110

4.5 Vertical energy conversion terms as functions of radius and height in the simulated penumbra . . . . . . . . . . . . . . . 111

4.6 Height dependence of the energy conversion terms in the simulated penumbra . . . . . . . . . . . . . . . . . . . 112

4.7 Variability of the CEF in a portion of the simulated penumbra . . . . . . 115 
4.8 Temporal average of the magnetic field vertical profiles, and the energy conversion by radial and vertical Lorentz force in CEF and NEF sources . 116

4.9 Temporal evolution of radial forces and radial mass flux in CEF sources . 116

4.10 Average contributions from the different terms of the induction equation to the radial and vertical field components at the NEF and CEF sources . . 120

4.11 Average contributions from the different terms of the induction equation to the vertical field component at the CEF sinks . . . . . . . . . . . . 121

4.12 Field-line connectivity and associated velocity and pressure perturbations in the simulated penumbra . . . . . . . . . . . . . . . . 122

5.1 Location of the LFPs on the continuum image of the center-side penumbra of the main sunspot in AR 10930. . . . . . . . . . . . . . . . . 130

5.2 Observed Stokes profiles in two LFPs . . . . . . . . . . . . . . . 132

5.3 Scatter-plots of SPINOR 2D inversions vs Zeeman and COG methods in the LFPs . . . . . . . . . . . . . . . . . . . . . . . . 134

5.4 Four examples of observed Stokes $V$ profiles in the LFPs . . . . . . . . 135

5.5 Observed Stokes profiles in two selected LFPs and their best fits returned from different inversions . . . . . . . . . . . . . . . 136

5.6 Deconvolved Stokes profiles in two LPFs and their best fits returned from different inversions . . . . . . . . . . . . . . . . . 137

5.7 Scatter-plot of magnetic field inclination in the LRF vs the LOS velocity according to the SPINOR 2D inversions in the LFPs . . . . . . . . . . . 140

5.8 A set of emergent synthetic Stokes profiles in the MURaM sunspot simulation at the location of a supersonic downflow in the tail of a CEFcarrying filament . . . . . . . . . . . . . . 141 



\section{List of Tables}

3.1 Geometrical properties of the filaments in different sectors of the penumbra of NOAA AR $10930 \ldots \ldots$. . . . . . . . . . . . 85

5.1 Results of Zeeman and center-of-gravity (COG) methods in two LFPs . . 131

5.2 Results of different height-dependent inversions applied to the observed and deconvolved Stokes profiles in two LFPs . . . . . . . . . . . . 144

5.3 Results of different height-independent inversions applied to the observed and deconvolved Stokes profiles in two LFPs . . . . . . . . . . . 145 



\section{Zusammenfassung}

Die Untersuchung von großskaligen Strömungen in Penumbren von Sonnenflecken in der Photosphäre ist essentiell, um den Energietransport in Sonnenflecken besser zu verstehen und die Helligkeit der Penumbra zu erklären.

Die wichtigste und charakteristische Strömung in einer gut ausgeprägten Penumbra ist der Evershed flow, eine nahezu horizontale, radial nach außen gerichtete Gasströmung entlang penumbraler Filamente. In sehr seltenen Fällen können einige penumbrale Filamente eine nach innen gerichtete Gasströmung beinhalten (sogenannte counter Evershed flows), die nur von begrenzter Dauer sind und deren Beschaffenheit noch unbekannt ist.

Diese Arbeit befasst sich mit der Ursache für sowohl radial auswärts als auch einwärts gerichteten Strömungen in einer gut ausgeprägten Penumbra.

Die Arbeit beginnt mit der Vorstellung einer einmaligen Beobachtung eines counter Evershed flows, der sich über einen großen Sektor der gut ausgeprägten Penumbra des Hauptsonnenfleckes der aktiven Region NOAA 10930 erstreckt. Zur Bestimmung der Höhenabhängigkeit der physikalischen Größen und Eigenschaften des Sonnenfleckes, wie zum Beispiel die dreidimensionale Struktur des Magnetfeldvektors, die Geschwindigkeit entlang der Sichtlinie, oder auch die Temperatur, werden Inversionen der spektro-polarimetrischen Daten des Solar Optical Telescope an Bord des japanischen Weltraumobservatoriums Hinode durchgeführt. Diese Inversionen basieren auf dem SPINOR-Code in seiner räumlich-gekoppelten Version. Die Übereinstimmungen und Unterschiede der magnetischen und dynamischen Strukturen in den penumbralen Filamenten mit auswärts und einwärts gerichteten Strömungen werden im Detail diskutiert um deren möglichen Ursachen zu ergründen. Dabei wird auch auf die fehlende Kenntnis über die geometrische Höhe der beobachteten Phänomene hingewiesen, die große Einschränkungen für die Interpretation einer Vielzahl von Phänomenen in der Photosphäre nach sich zieht. In diesem speziellen Fall verhindert diese Unkenntnis die Unterscheidung zwischen zwei rivalisierenden Erklärungen für die Ursachen des Evershed flows und des counter Evershed flows.

Der zweite Schwerpunkt liegt auf der Analyse hochaufgelöster Simulationen eines Sonnenfleckes. Diese Simulationen wurden mit dem MURaM-Code durchgeführt, der auf den Gleichungen für den Strahlungstransport und der Magneto-Hydrodynamik beruht. Sie reproduzieren die Filament-Struktur der Penumbra mit dem normalen Evershed flow, aber auch mit einigen, kurzlebigen counter Evershed flows in der Photosphäre. Sie offenbaren die beiden Strömungen zugrunde liegende, physikalische Struktur auf einer geometrischen Höhenskala. Dies ist ein entscheidender Vorteil gegenüber den Beobachtungen, die die Informationen nur über Flächen konstanter optischer Tiefe preisgeben. Diese Untersuchung belegt, dass der Evershed flow und der counter Evershed flow unterschiedliche Ursachen haben. Außerdem liefert sie eine Erklärung für die Kurzlebigkeit und das seltene Auftreten des counter Evershed flows in der Penumbra von Sonnen- 
flecken. Es wird gezeigt, dass der Evershed flow das Resultat einer Konvektionsströmung in einem stark geneigten Magnetfeld ist, während der counter Evershed flow eine feldlinienparallele Strömung ist, die von horizontalen Gasdruckgradienten angetrieben wird, und daher auch als Siphon-Strömung bezeichnet werden kann.

Abschließend diskutiere ich die Zuverlässigkeit von Inversionen in Regionen mit abwärts gerichteten Überschallströmungen, die Feldstärken in der Penumbra von — mehr als $7 \mathrm{kG}$ - liefern und mit den Senken des counter Evershed flows an der inneren Grenze der Penumbra assoziiert sind. Dazu führe ich verschiedene Tests mit einfachen Techniken zur Bestimmung der Magnetfeldstärke durch und wende unterschiedliche Inversionsmethoden an. Diese hohen Magnetfeldstärken wären, falls korrekt, die höchsten jemals direkt auf der Sonne gemessenen Feldstärken. 


\section{Summary}

The analysis of photospheric large-scale flows in sunspot penumbrae is a key ingredient for understanding how the energy is transported in sunspots and to explain the penumbral brightness.

The most prominent and characteristic flow in a well-developed penumbra is the Evershed flow, which is an almost horizontal and radial outflow of gas confined along the penumbral filaments. In very rare occasions, some penumbral filaments can instead host inflows (counter-Evershed flows), which are transitory events and, of still unknown nature.

This thesis is concerned with the origin of both outflows and inflows in well developed penumbrae.

Firstly, I introduce a unique observation of counter-Evershed flows occurring in a large sector of the well-developed penumbra of the main sunspot in active region NOAA 10930. In order to infer the height stratification of the physical quantities and the properties of the sunspot, such as the three-dimensional structure of the magnetic field vector, the lineof-sight velocity, and the temperature, inversions of the spectropolarimetric data obtained with the Solar Optical Telescope on board the Japanese Hinode space observatory were carried out by using the spatially coupled version of the SPINOR inversion code. The similarities and differences of the magnetic and dynamic structures between the different types of penumbral filaments (outflow- and inflow-carrying filaments) are discussed in detail to explain their possible nature. The lack of knowledge of the geometric height of the observed phenomena is pointed out. Basically, these cause limitations for the interpretation of any photospheric phenomena and, in particular, do not allow distinguishing between rival explanations for the drivers of the Evershed and counter-Evershed flows.

Secondly, I analyze a high-resolution sunspot simulation based on the MURaM radiation-magneto-hydrodynamic code, which reproduces a filamented penumbra with a normalEvershed outflow and a number of transient regions with counter-Evershed flows at photospheric heights. The simulations revealed the underlying physical structure of both flows on a geometrical height scale. This is an important advantage over the observations, whose current analysis techniques generally provide information on constant optical depth levels only. The results of this investigation reveal different natures for the normal- and the counter-Evershed flows, and a possible explanation for the relatively short lifetimes and the rare occurrence of counter-Evershed flows in sunspot penumbrae. We found that the Evershed flows occur due to overturning convection in a strongly inclined magnetic field while the counter-Evershed flows are field-aligned flows consistently driven by horizontal gas pressure gradients and thus, can be well described as siphon flows.

Finally, I investigate and discuss the reliability of inversion results returning very large penumbral field strengths — larger than $7 \mathrm{kG}$ —in supersonic downflow regions, which 
are associated with the counter-Evershed flow sinks at the inner penumbral boundary. By using simple field strength estimation methods and different inversion techniques, I test the validity of our inversion results. The observation of such unusually large fields, if real, would correspond to the largest field strengths ever directly measured anywhere on the Sun. 


\section{General Introduction}

The proximity of the Sun to the Earth makes it the most special star in the universe for humankind. It is not only responsible for the existence of life on Earth but also provides us with a unique opportunity to study a star in great detail. In particular, the Sun's surface magnetism and dynamics can nowadays be studied at high spatial resolution, the largest solar telescopes are at present able to resolve solar structures down to a size of only 50 $\mathrm{km}$.

The discovery of the Zeeman effect (Zeeman 1897) gave birth to the exploration of astrophysical magnetic fields. It was actually in a sunspot where the first measurement of an extraterrestrial magnetic field was made (Hale 1908). However, the existence of a global magnetic field in the Sun with a dipolar-like shape was already noticed by George Ellery Hale in 1893 through the observation of the geometrical appearance of the solar corona during eclipses.

Currently, there are still strong debates regarding how and where the solar magnetic field is generated (e.g., Brandenburg 2005). On the one hand, it is argued that the magnetic field of the Sun is generated by a dynamo acting close to the boundary between the radiative zone and the convection zone (e.g., Spiegel and Weiss 1980, Golub et al. 1981, Choudhuri 1990) due to the formation of a radial shear layer resulting from the solar differential rotation, the so-called tachocline (e.g., Spiegel and Zahn 1992). On the other hand, a convection driven dynamo operating in a distributed manner in the entire convection zone has also been considered (e.g., Brandenburg 2005). Yet, another idea states that the solar dynamo might play an important role within the uppermost $35 \mathrm{Mm}$ of the Sun (Dikpati et al. 2002, Mason et al. 2002, Brandenburg 2005) due to the existence of a near-surface layer of negative radial shear (e.g., Howe et al. 2000, Thompson et al. 2003) which appears to be stronger near $30^{\circ}$ latitude (which is where the largest magnetic activity in the beginning of a solar cycle occurs) than the radial shear at the tachocline (e.g., Benevolenskaya et al. 1999).

The magnetic flux present in the convection zone is then believed to become unstable and buoyant, so that it is partially transported towards the external layers up to the photosphere, where it can expand towards the chromosphere and afterwards towards the corona, to be later partly carried into the interplanetary medium by the solar wind. However, many of the fundamental processes involved in the origin, transport, emergence through the surface, and the evolution of the small- and large-scale solar magnetic fields are still not well understood and remain controversial (see e.g. reviews by Charbonneau 2005, 2010, 2014, Brun et al. 2015).

One of the reasons is that the solar magnetic field is much more complex than a simple dipole resembling a big magnet with fixed north and south poles. Instead, the topology of the solar magnetic field is constantly changing in time. The small-scale field generally 
changes dramatically over short time spans, while the global field changes in a cyclical manner over a period of 22 years.

Another reason is that we are only able to directly measure the surface field (typically by means of the Zeeman effect, which is best observed in the photospheric spectral lines), while the strength and structure of the magnetic field above and beneath the surface is poorly known: on the one hand, the subsurface magnetic field is impossible to measure, albeit indirect methods for the sub-photospheric large-scale fields have been and are being explored within the scope of local helioseismology (e.g., Gizon and Birch 2005, Gizon et al. 2010, and references therein); on the other hand, the topology of the magnetic field in the corona is generally extrapolated from photospheric magnetic field measurements (using potential or force-free fields models) or depends on proxies such as the EUV images of coronal loops, among others.

Because of all these limitations, our conception of the Sun's magnetic field largely depends on its magnetism on the surface as well as on theoretical calculations such as numerical simulations within the framework of magnetohydrodynamics (MHD).

The scope of this introduction is narrowly focused on the solar photosphere and the different ways of manifestation of its magnetic fields. However, the fundamental processes taking place in the solar interior as well as in the external atmospheric layers deserve a place at the outset of this section. Thereupon, I concentrate on a general overview of magnetism in the solar lower atmosphere and present a brief description of some current tools and techniques for the analysis of solar magnetic field measurements in the photosphere. Finally, I describe the MURaM radiative and magnetohydrodynamic (MHD) code (Vögler et al. 2005), which is a widely used code to simulate the macro-physics related to the interaction and dynamics of flows and magnetic fields in the photosphere and interior of main-sequence stars and, in particular, of the Sun.

\subsection{The Sun}

\subsubsection{Internal structure}

The Sun is a middle aged star, classified as a G2V main-sequence star based on its spectral characteristics and on its effective surface temperature of $\sim 5800 \mathrm{~K}$. It is the main source of energy in our solar system and by far the most important factor making life possible on Earth.

It comprises of a core that extends from its center to $\sim 0.25$ of the solar radius $\left(R_{\odot} \approx\right.$ $6.9 \times 10^{5} \mathrm{~km}$ ) which contains roughly $34 \%$ of the star's mass at a temperature of $\sim 1.5 \times 10^{7}$ $\mathrm{K}$ and generates most of the energy released by the Sun (see Fig. 1.1 for a sketch of the internal structure of the Sun).

Due to the high temperatures and plasma densities $\left(\sim 150 \mathrm{~g} \mathrm{~cm}^{-3}\right)$, quantum tunneling is possible in the core so that the energy production is made by nuclear reactions which consume hydrogen to form helium, photons, and neutrinos through the proton-proton chain. Unlike the neutrinos, which can escape the core almost undisturbed in spite of the extremely high plasma density, the photons interact with matter so strongly that they can be scattered by another particle almost immediately after being emitted, thus escaping the Sun within time-scales of the order of $10^{5}$ years. 


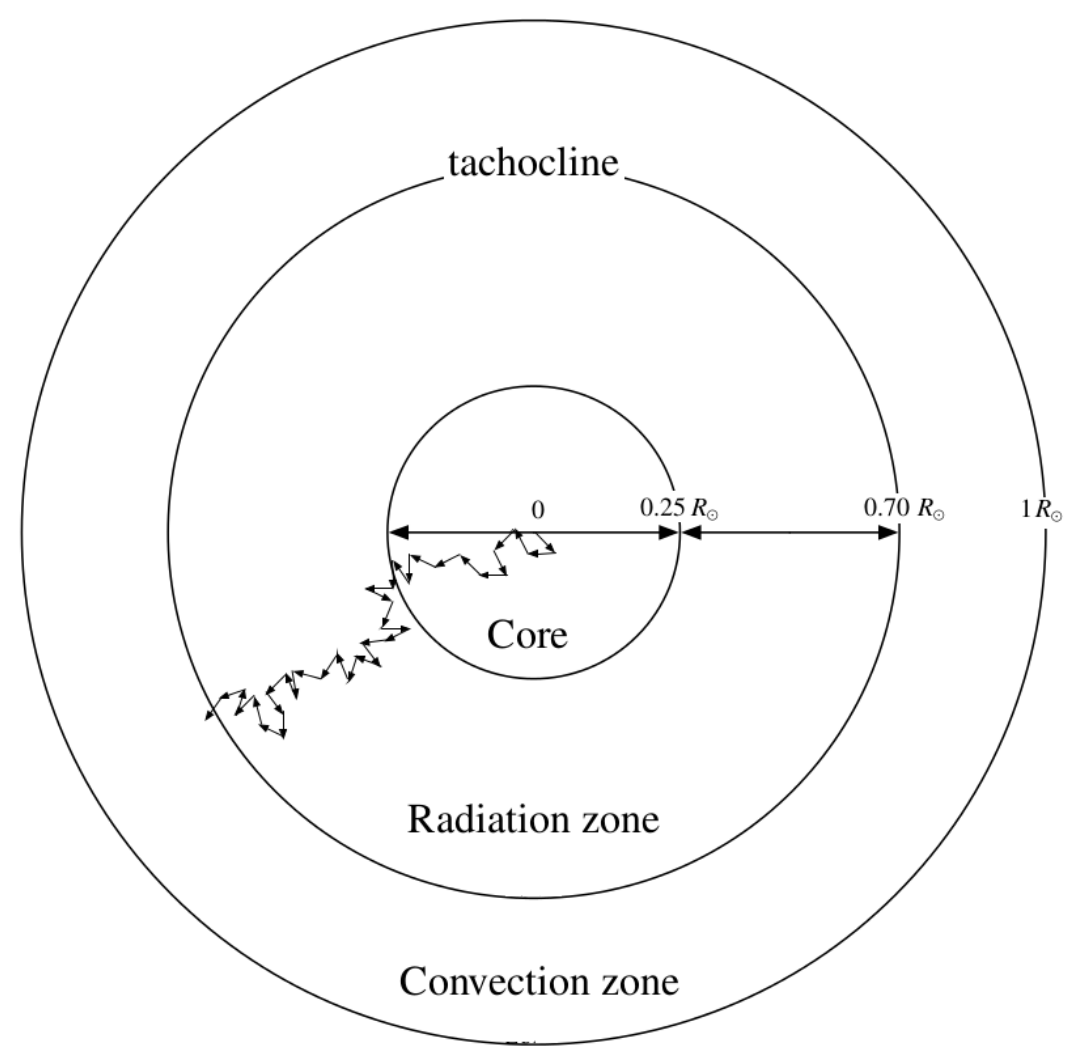

Figure 1.1: Sketch illustrating the internal structure of the Sun and the photons random walk from the core through the radiation zone in a simplified version. Adapted from Walker (2006).

After reaching the outer edge of the core, energy transport by radiation continues up to $\sim 0.70 R_{\odot}$ within the radiation zone, but the mean free path of the photons is here on average larger than in the core due to the radial decrease of the density and the temperature (from about 20 to $0.2 \mathrm{~g} \mathrm{~cm}^{-3}$ and from roughly $7 \times 10^{6}$ to $2 \times 10^{6} \mathrm{~K}$, respectively, across this zone).

Radiative energy transport is thereafter replaced by convection as the main form of energy transport from the bottom of the so-called convection zone (above $\sim 0.70 R_{\odot}$ ) up to the photosphere, where the rising hot plasma gets cooled down at the surface by radiative losses. As a consequence of the radiative cooling, the photons can escape the Sun and the cool gas sinks back to the sub-surface layers forming characteristic flow patterns known as convective cells, which nonetheless involve intense turbulent fluid flows (e.g., Tkaczuk et al. 2007).

Perhaps one of the most important achievement during the last century, regarding the solar interior, was the mapping of the internal rotation of the Sun by means of helioseismology (Brown et al. 1989). Close to the boundary between the radiation zone and the convection zone lies a boundary layer that delimits different regimes of rotation: the innermost $0.70 R_{\odot}$ rotates almost rigidly like a solid body, while the convective external layers rotate differentially as a fluid. This differential rotation is almost purely latitudinal in the main convection zone (e.g., Dikpati and Gilman 2001) implying that the largest radial velocity gradients occur in the transition layer between the two regimes, which is called 


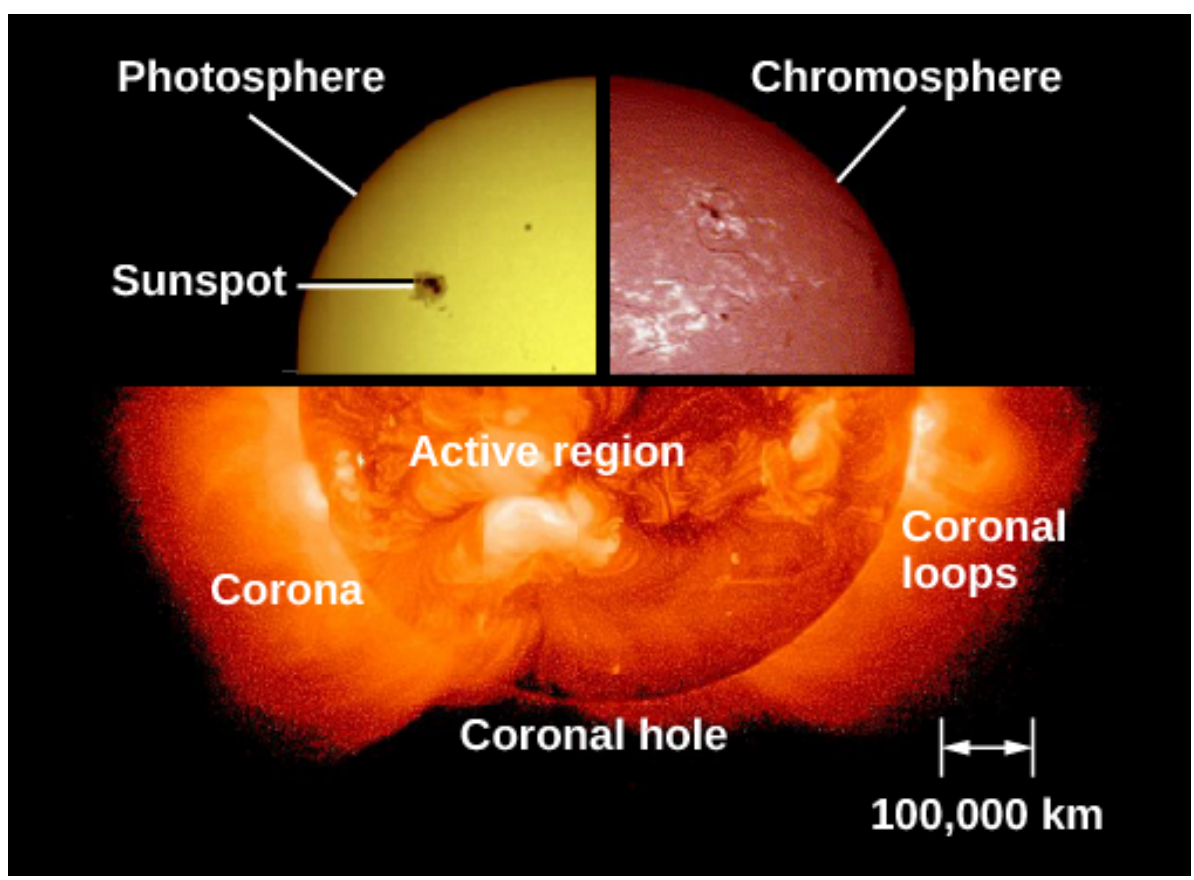

Figure 1.2: The solar atmosphere. Composite image showing three different layers of the solar atmosphere: the photosphere or surface of the Sun in white light; the chromosphere, imaged in $\mathrm{H}-\alpha$; and the corona as seen in X-rays. Credits: ESA/NASA SOHO and Yohkoh.

the tachocline. Thus, the tachocline plays an essential role in solar dynamo theories given that it represents a region with a very large shear profile, which can lead to the generation of large-scale magnetic fields in the Sun (see e.g., Brown et al. 1989, Charbonneau et al. 1999, Miesch 2005, Rempel 2005).

\subsubsection{Solar atmosphere}

The solar atmosphere is the outer and visible part of the Sun. It is composed of three main layers, namely the photosphere, the chromosphere, and the corona (Fig. 1.2). All these atmospheric layers posses different physical properties, but are dynamically and magnetically coupled together (e.g., Wedemeyer-Böhm et al. 2009).

The solar surface or lower photosphere is the upper boundary of the convection zone where the granular pattern of the convective gas is visible as a brightness variation due to different temperatures of the gas (bright regions - hot uprising gas, and dark lanes cooler sinking gas). The plasma density decreases rapidly above the photosphere (Fig. 1.3) and the mean free path of the photons becomes large enough that most of the photons reaching this point can escape the Sun. The bulk of the electromagnetic energy escaping the Sun is emitted in the visible part of the solar spectrum, and it peaks at a wavelength close to $500 \mathrm{~nm}$ (Fig. 1.4). Thus, the solar surface is conventionally placed at the height where the continuum optical depth at $500 \mathrm{~nm}$ is unity, and the photosphere extends over roughly $500 \mathrm{~km}$ above the solar surface.

The thick chromospheric layer (which is on average $1500 \mathrm{~km}$ thick but with strong variations) starts close to the temperature minimum $\left(T_{\min } \sim 4500 \mathrm{~K}\right)$ and spans up to the 


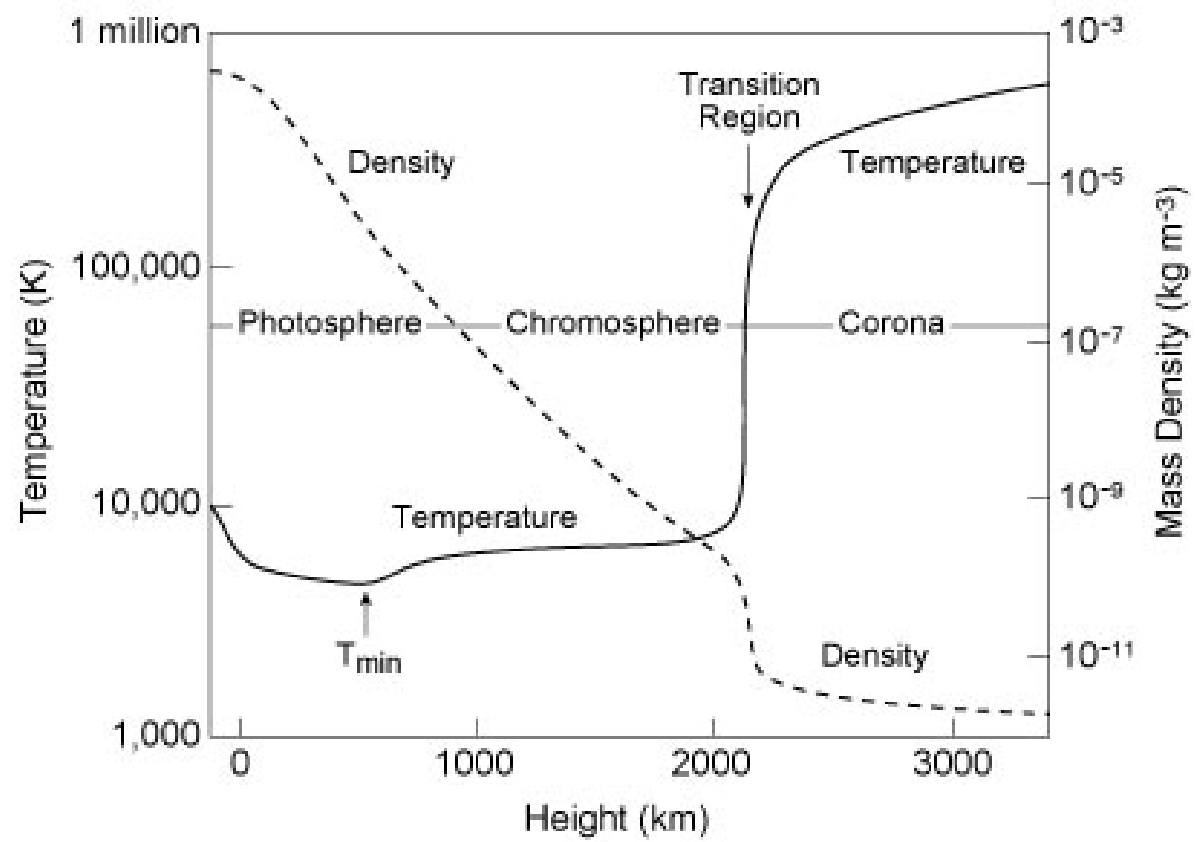

Figure 1.3: Spatially averaged temperature (solid line) and density (dashed line) as functions of height throughout the different layers of the solar atmosphere. Credit : Eugene Avrett, Smithsonian Astrophysical Observatory.

transition region, which is characterized by a huge jump of the plasma temperature (from about $10^{4} \mathrm{~K}$ to $\sim 10^{6} \mathrm{~K}$ ) and a pronounced drop of the plasma density (Fig. 1.3). This part of the atmosphere has sufficiently low density that collisions are rare and atoms no longer satisfy Saha and Boltzmann statistics. Consequently, local thermodynamic equilibrium (LTE) is not satisfied during the formation of most chromospheric lines (e.g., Vernazza et al. 1976, Tziotzio et al. 2001). The chromosphere is magnetically and dynamically very complex since the magnetic field changes from non force-free in the lower chromosphere to nearly force-free in the upper chromosphere. Besides, the temperature unexpectedly increases after the temperature minimum. All these phenomena occurring in the solar chromosphere are the key for understanding its connection with the photosphere and with the higher layers, and make the chromosphere hard to model, so that it remains a very enigmatic layer.

The transition region also marks the beginning of the solar corona, where the matter reaches highly ionized states due to the very high temperatures (around 1 million Kelvin), causing a large part of its radiation to fall into the extreme ultraviolet (EUV) and Xrays parts of the spectrum, but its entire spectrum covers a wide range of wavelengths, including also radio emissions. Both, the chromosphere and the corona, are permeated by magnetic fields which due to the low plasma-beta dominate the physical processes therein. They present a rich variety of complex and violent phenomena resulting from the interaction between the plasma and the magnetic field.

The mechanisms responsible for the heating of the chromosphere and the solar corona are still poorly understood, but it is generally recognized that the magnetic field plays an important role in this problem (e.g., Klimchuck 2006). As a consequence of a difference in pressure in corona and interstellar medium, the particles in the coronal plasma can 


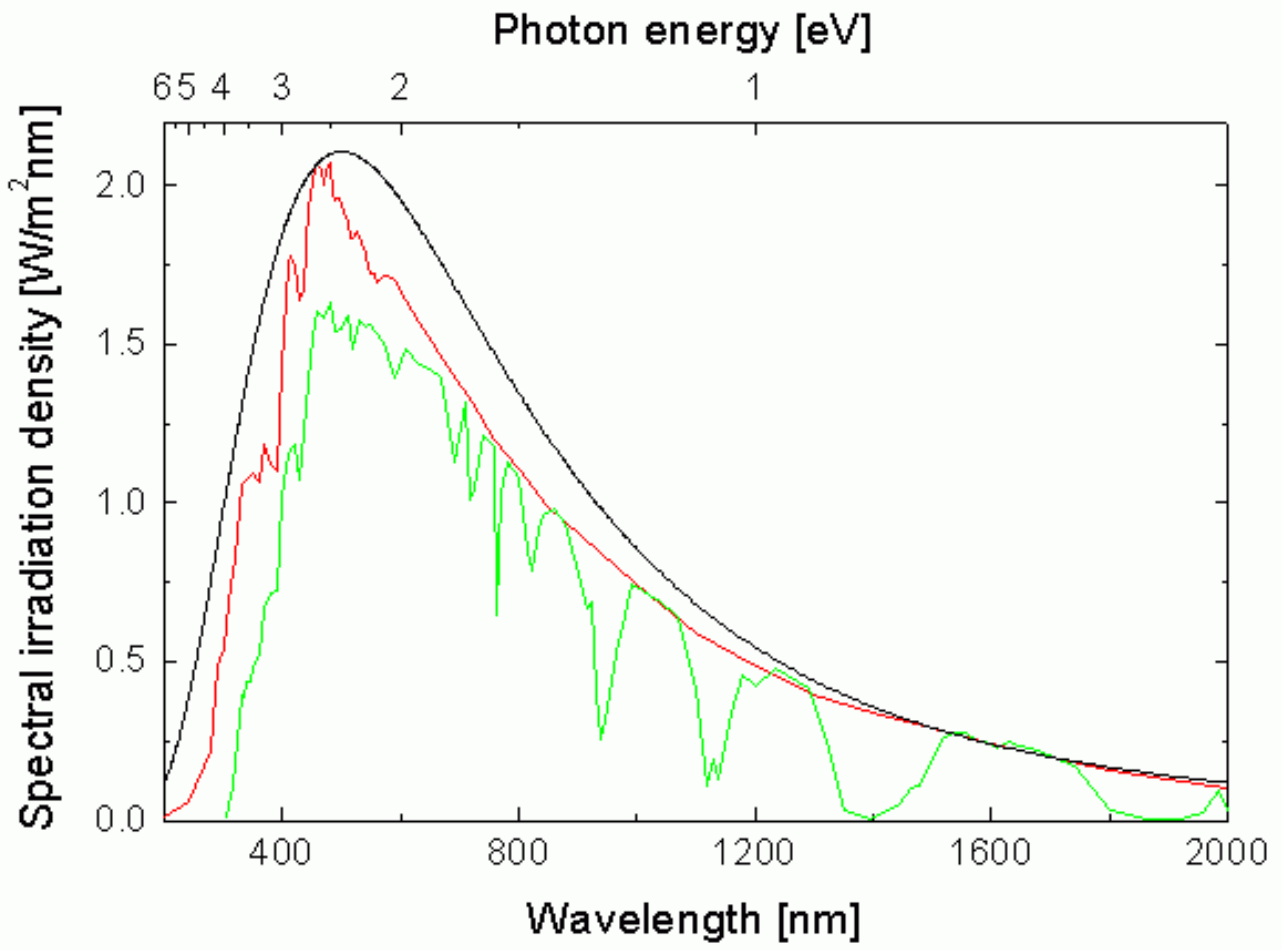

Figure 1.4: Comparison of spectral irradiation densities: black body radiation at $5800 \mathrm{~K}$ (black curve), AM0 (air mass zero) irradiation in earth orbit (red curve), AM1.5 irradiation at sea level in Central Europe (green curve). From: https://www.tfp.ethz.ch/Lectures/pv/spectrum.html

continuously escape into the interplanetary space (Parker 1958). Hence, the solar corona does not have a well defined outer boundary but it instead smoothly connects to the solar wind

\subsection{Solar lower atmosphere magnetism}

Magneto-convection is the interaction between the convective plasma motions and the magnetic field. When the magnetic field is sufficiently strong so that the Lorentz force surpasses the force exerted by the moving plasma, then the magnetic field rules the plasma motion across the field direction and can even inhibit convection. If the opposite occurs, then the convective motions can stretch the magnetic field or modify its topology. Moreover, turbulent convective flows can lead to the strengthening of the field, which is the so-called 'dynamo action' (Weiss 1966, Vögler and Schüssler 2007). Thus, in the solar photosphere and in particular in an active region, magneto-convection has different regimes given that it is permeated by magnetic fields on a wide range of scales, which produce a variety of visible features. Some of them are highlighted on the G-band image in Figure 1.5.

The darkest regions indicate large concentrations of magnetic flux where the convective motions are significantly suppressed. Sunspots are the largest dark regions on the 


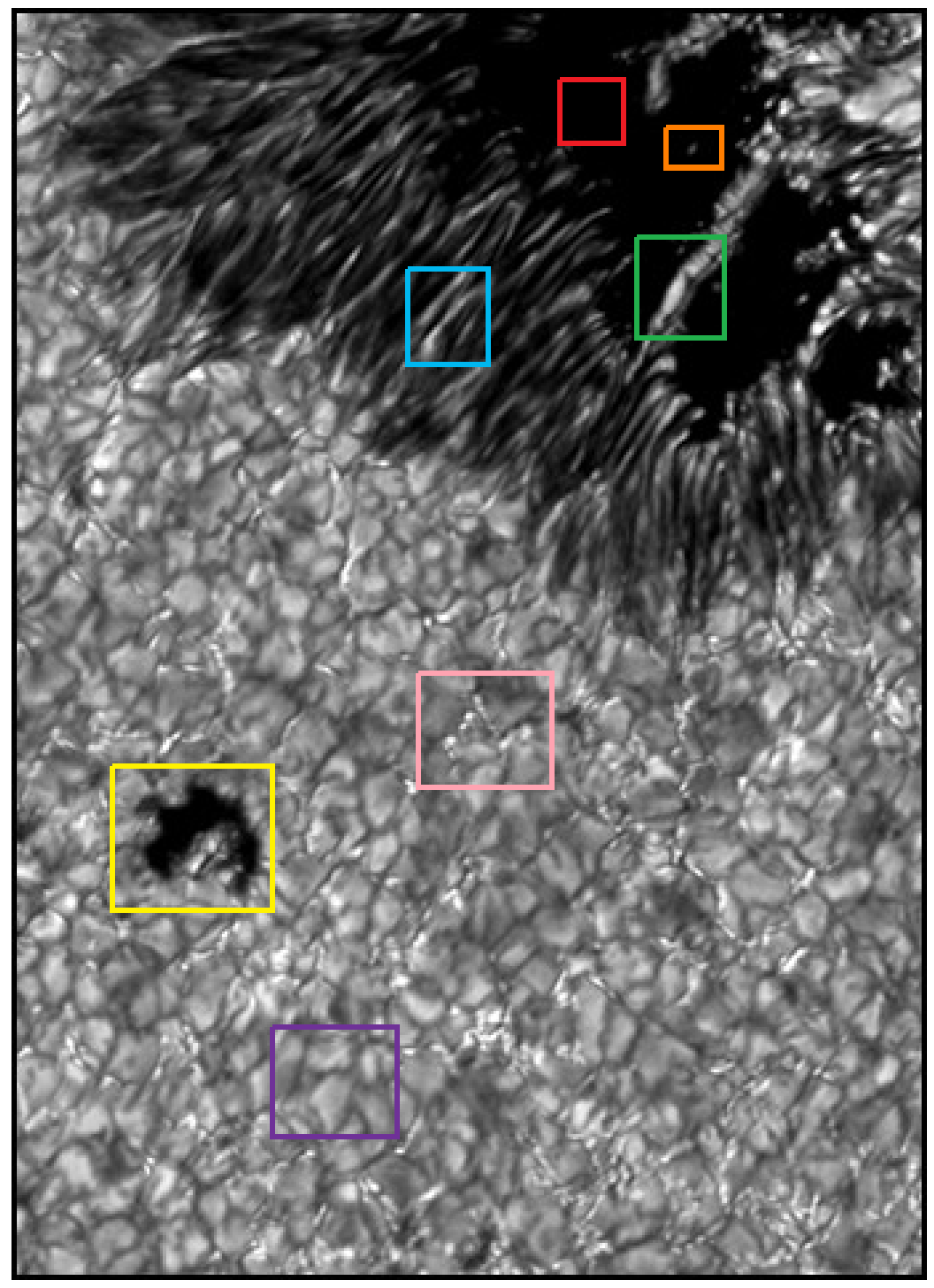

Figure 1.5: Different regimes of solar magneto-convection in a G-band image taken from the Vacuum Tower Telescope (VTT). Features enclosed by colored squares are: sunspot's dark umbral core (red), umbral dots (orange), light bridge (green), penumbral filaments (cyan), plage (pink), pore (yellow) and quiet Sun (purple). The red, orange and green squares indicate parts of the umbra. Credits: KIS/VTT, Observatorio del Teide, Tenerife. 
solar photosphere and host magnetic fields of the order of thousands of Gauss, causing a temperature decrease of up to $2000 \mathrm{~K}$. They are usually associated with bipolar regions and they most commonly appear in pairs of opposite magnetic polarities.

Sunspots are well structured regions, with a dark core or 'umbra' where the magnetic field orientation is mostly vertical and its strength is the largest, and a less dark region called the 'penumbra' which generally completely surrounds the umbra and where the magnetic field is filamented, strongly inclined, and with an on average radially decreasing strength (see e.g., Solanki 2003, Schlichenmaier 2009, Borrero and Ichimoto 2011, and Chapter 2 for a review on the fine-scale and global structure of sunspots). However, sunspots can also appear with a partial penumbra or completely without of it, in which case they are called 'pores'. Moreover, sometimes sunspots are formed by multiple umbrae separated by bright lanes known as light bridges. Also, multiple bright points are frequently observed inside the umbra (the so-called umbral dots). Both phenomena are usually interpreted as traces of convection.

In the vicinity of sunspots, the intergranular lanes can appear filled with bright points which are referred to as 'plage regions' or 'faculae'. In plage regions, the convective granules are generally smaller, live for longer periods than in the quiet Sun (Dunn and Zirker 1973), and harbor large magnetic flux concentrations (kiloGauss fields) which are often considered to be structured as flux tubes (e.g., Solanki 1993). Different processes are considered in order to explain such flux concentrations. Firstly, the so-called flux expulsion process (Parker 1963), in which the convective flows compress the field so that the magnetic energy approaches the kinetic energy of the gas (e.g., Danilovic et al. 2010), can produce field concentrations of a few $100 \mathrm{G}$. In addition, the so-called convective collapse instability (e.g., Spruit 1979, Venkatakrishnan 1986) driven by radiative cooling of the gas trapped between the field lines, can achieve the further strengthening of the field to the observed kiloGauss values.

Magnetic field concentrations on the solar surface are also present on smaller scales (to our present knowledge, as small as the diffraction limit of the largest telescopes to date). The apparently "magnetically quiet" convective environment, known as quiet Sun, was initially believed to be nearly field-free. Nonetheless, weakly magnetized regions were discovered by Livingston and Harvey (1971) in the interiors of supergranular convective cells (internetwork). When the magnetic flux per unit area is small enough, then it is carried by the convective motions and concentrated in the downflow regions at the boundaries of large scale convective cells or supergranules (network). The magnetic field in the network has actually field strengths of the order of kiloGauss (e.g., Stenflo 1973, Wiehr 1978, Solanki and Stenflo 1984, Stenflo and Harvey 1985, Solanki et al. 1987, Grossmann-Doerth et al. 1996). Internetwork and network magnetic fields have been estimated to contribute to the total solar magnetic flux with $\sim 10^{26} \mathrm{Mx}$ per day, which is about 4 orders of magnitude larger than the contribution of active regions (Zhou et al. 2013).

Thus, magnetic fields are ubiquitous on the solar surface and have a direct impact on the physical conditions of the higher atmospheric layers, the chromosphere and corona, in a broad range of scales. The importance of inferring magnetic fields in the photosphere lies not only in the understanding of how the energy transport occurs from the surface to the corona, but also in space weather forecasting to avoid damage to our technical and natural environments, as well as in more fundamental physics topics such as magnetohy- 
drodynamics, dynamo theory and atomic physics.

\subsection{Photospheric magnetic field measurements}

Except for the solar wind, where in-situ measurements of the interplanetary magnetic field have been possible from $~ 0.3$ AU outward by means of magnetometers on board spacecrafts (e.g., Mariani et al. 1979, Zurbuchen and Richardson 2006), magnetic fields in the solar atmosphere can only be inferred through remote-sense observations.

In particular, in the solar lower atmosphere several methods can be used to estimate the properties of the plasma and the magnetic field since this information is encoded in the spectral lines. However, extracting such information from the radiation emitted from the solar surface is usually a nontrivial task that implies the use of advanced instrumental capabilities as well as of multiple and sophisticated analysis techniques.

\subsubsection{Doppler and Zeeman effect}

Practically all the observed solar radiation originates in the photosphere and produces a spectrum that resembles that of a black body at a temperature of about $5800 \mathrm{~K}$ (Fig. 1.4). Most of this emitted electromagnetic energy corresponds to the visible and near-infrared parts of the spectrum.

In the solar photosphere, the temperature of the plasma is sufficiently low so that recombination occurs and therefore, in addition to protons and electrons, the gas is also composed of ions, neutral atoms, and some molecules, which have multiple energy levels. Consequently, the photons can be absorbed and emitted at characteristic wavelengths (with those wavelengths corresponding to energies equal to the energy difference between two electron energy levels in an atom or molecule), thus producing a solar spectrum that contains a huge number of well-defined spectral lines. Since the plasma temperature in the photosphere decreases upwards (and the conditions for local thermodynamic equilibrium are approximately satisfied - see below), its spectrum is dominated by absorption lines.

Because of the ubiquitous temperature gradients in the photosphere global thermodynamic equilibrium is not possible. However, the solar photosphere generally satisfies the conditions of local thermodynamic equilibrium (LTE), i.e., the thermodynamic state of a given atomic/ionic/molecular specie at a certain place can be well described with a single temperature, which at the same time is sufficient to represent the velocity distribution function of the gas particles at such place as a Maxwellian, the population of the atomic states with the Saha and Boltzmann equations, and the local radiation field with the homogeneous and isotropic black-body form given by the Kirchhoff-Planck function (see e.g., Stix 2002).

Thus, for spectral lines that are formed under LTE conditions, the velocity distribution function of the gas particles (for a given specie) produces the broadening of the spectral lines such that the root-mean-square width or Doppler width, $v_{D}$, can be related to the temperature, $T$, as: 


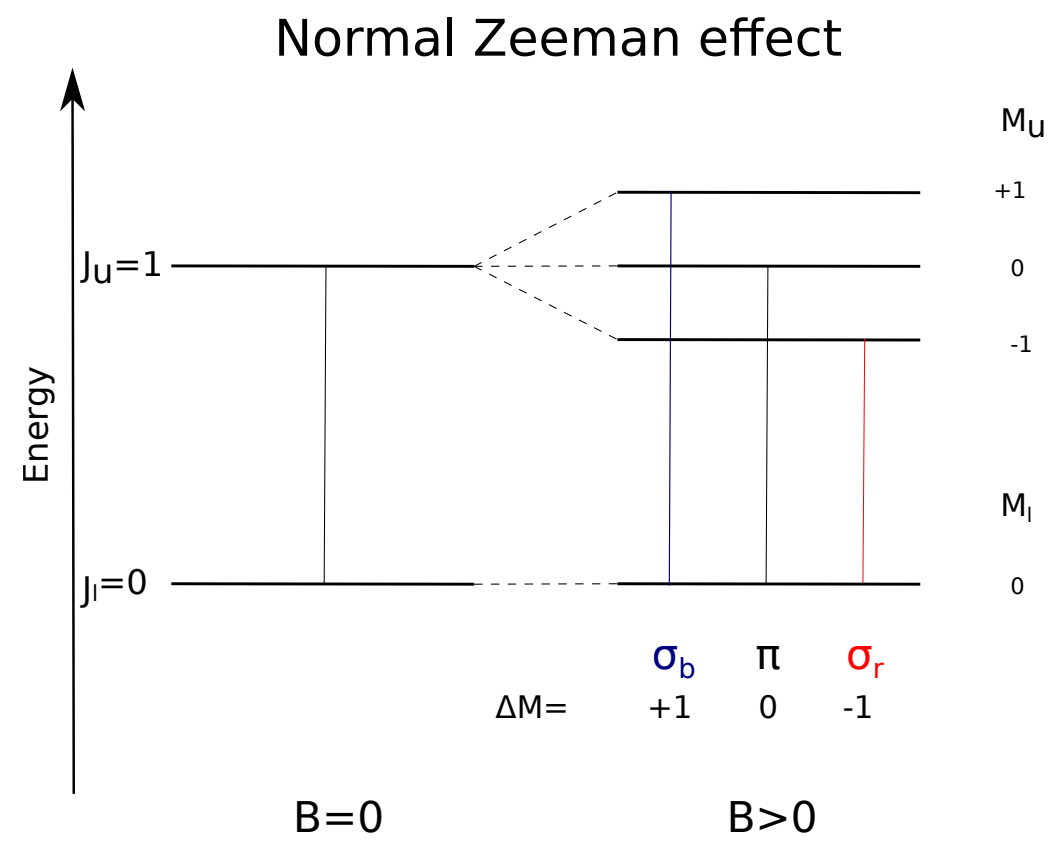

Figure 1.6: Normal Zeeman effect: Example of an atomic transition occurring between two energy levels, in which the lower level has $J_{l}=0$ and the upper level has $J_{u}=1$, in the absence of an external magnetic field (left) and in the presence of an external field (right) which produces the splitting of the upper level into $2 J_{u}+1$ different sublevels.

$$
v_{D}=\sqrt{\frac{2 k T}{m}+v_{M I C}^{2}}=\frac{c \Delta \lambda_{D}}{\lambda_{0}}
$$

where $k$ is the Boltzmann constant, $m$ is the mass (at rest) of the atom, $v_{M I C}$ is a parameter that accounts for motions on smaller scales than the mean free path of the photons and is called the microturbulence velocity, $\lambda_{0}$ is the central wavelength of the spectral line, $c$ is the speed of light, and $\Delta \lambda_{D}$ is the Doppler width in terms of wavelength.

On top of that, the gas emitting the photons in the photosphere is in constant motion, mainly due to convection. Such convective motions produce a non-relativistic Doppler effect which can be seen as an additional broadening of the spectral lines when the wavelength shifts produced by up- and downflows are superposed, or as asymmetric spectral lines when velocity gradients exist.

Spectral line broadenings can also be produced by the presence of magnetic fields in the solar surface. This is a result of the Zeeman effect, which is the splitting of the spectral lines due to modifications in atomic structure produced by an external magnetic field. If such an external magnetic field is uniform at the atomic scale, then the Hamiltonian of the atomic system is given by (Baym 1969):

$$
H=H_{0}+H_{B}
$$

where $H_{0}$ is the Hamiltonian of the unperturbed atomic system in the absence of an external magnetic field and the additional term $H_{B}$ is the magnetic Hamiltonian, which in the weak field regime of the linear Zeeman effect (spin-orbit coupling) is given by: 


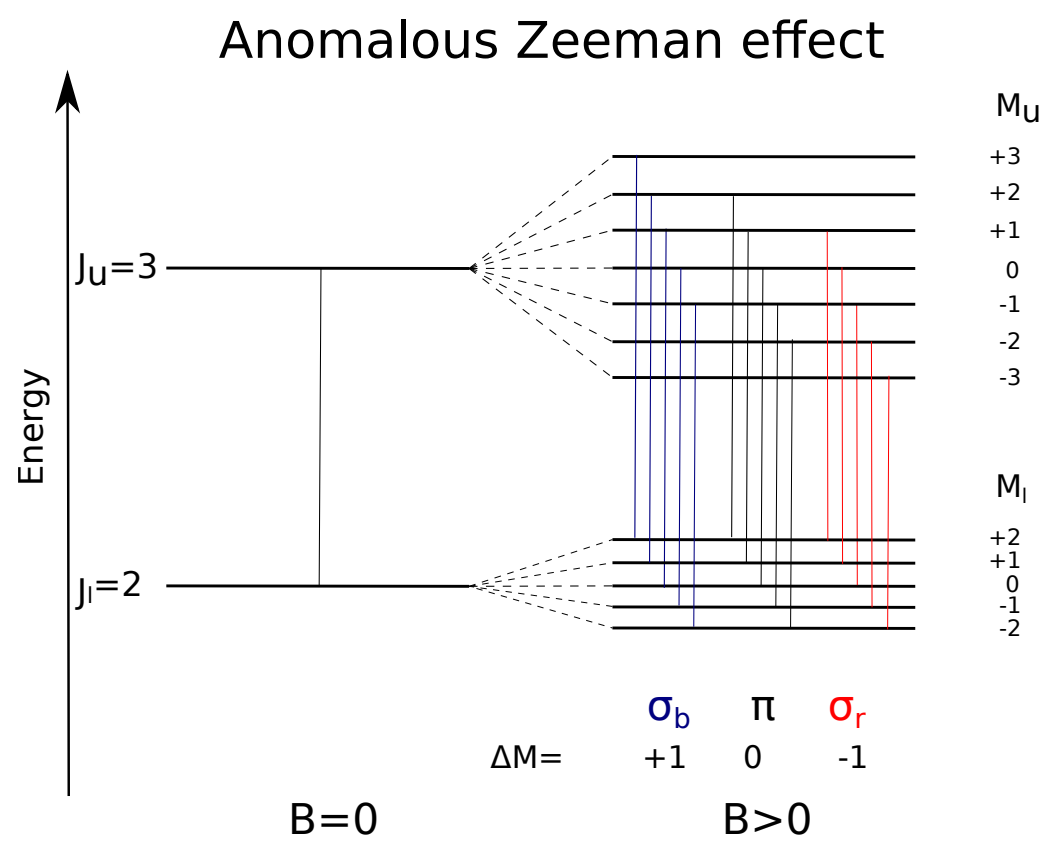

Figure 1.7: Anomalous Zeeman effect: Example of an atomic transition occurring between two energy levels, in which the lower level has $J_{l}=2$ and the upper level has $J_{u}=3$, in the absence of an external magnetic field (left) and in the presence of an external field (right) which produces the splitting of the lower and upper levels into $2 J_{l}+1$ and $2 J_{u}+1$ different sublevels, respectively.

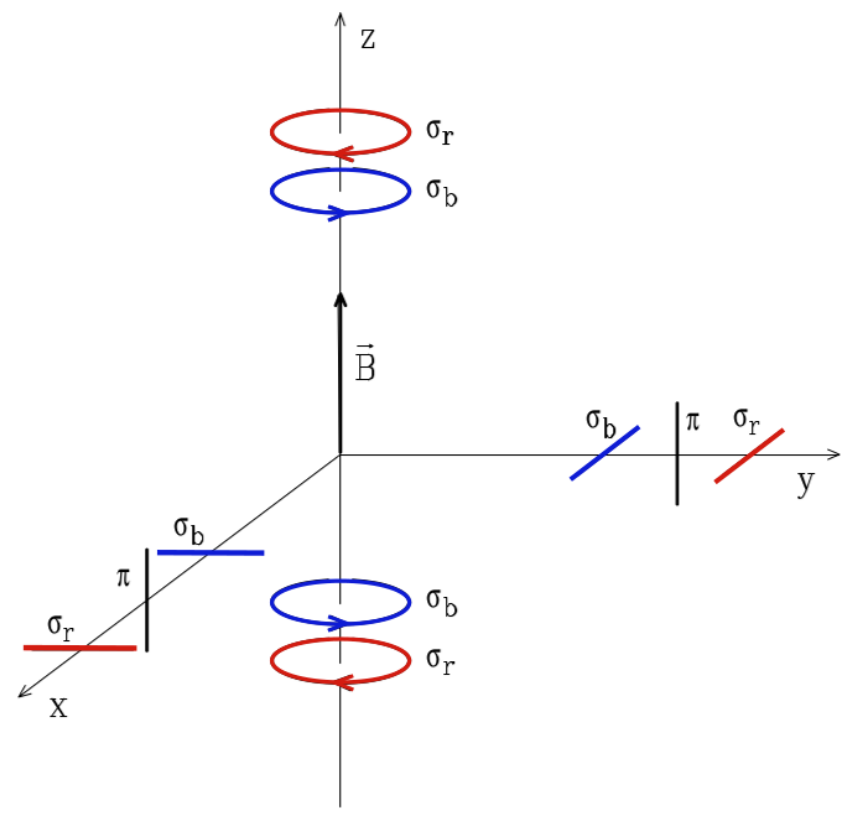

Figure 1.8: Polarization of the different Zeeman components in an emission line for linesof-sight parallel and antiparallel to the magnetic field vector, as well as in the plane perpendicular to $\vec{B}$ (x-y plane). Adapted from Landi Degl'innocenti and Landolfi (2004). 


$$
H_{B}=\frac{e \hbar}{2 m_{e} c}(\vec{L}+2 \vec{S}) \cdot \vec{B}=\mu_{B}(\vec{L}+2 \vec{S}) \cdot \vec{B}
$$

with $e$ being the absolute value of the electron charge, $\hbar=h / 2 \pi$ the reduced Planck constant, $m_{e}$ the electron mass, $c$ the speed of light, $\vec{L}$ and $\vec{S}$ the total orbital angular momentum and the total spin angular momentum respectively, and $\vec{B}$ the external magnetic field vector. $\mu_{B}=9.27 \times 10^{-21} \mathrm{erg} \mathrm{G}^{-1}$ is the so-called Bohr magneton.

Nonetheless, if the external magnetic field is strong enough so that the splitting of the energy levels is of the same order as the energy separation between two degenerate states, then the orbital angular momentum, $L$, and the spin angular momentum, $S$, couple more strongly to the external magnetic field than to each other, and therefore precess independently around the external magnetic field ( $L S$-decoupling). The splitting of the energy levels in the strong-field scheme can be well described by the Paschen-back effect where, unlike in the Zeeman effect, the $J$ quantum number is no longer a constant of motion (a detailed discussion is provided by, e.g., Landi Degl'innocenti and Landolfi 2004).

The eigenvalues of the total Hamiltonian in equation 1.2 can be found by applying first order time-independent perturbation theory (e.g., Landi Degl'innocenti and Landolfi 2004 , and references therein), which leads to the splitting of the energy levels into $2 J+1$ magnetic sublevels of slightly different energies as follows:

$$
E_{J, M}=E_{J}+\mu_{B} g M B . \quad(M=-J, \ldots, 0, \ldots, J)
$$

Here, $E_{J}$ is an eigenvalue of $H_{0}, M$ is the magnetic quantum number which is the projection of the total angular momentum $J$ along the direction of the magnetic field $(J \equiv L+S)$, and $g$ is called the Landé factor which in $L S$-coupling can be written as:

$$
g_{L S}=1+\frac{J(J+1)+S(S+1)-L(L+1)}{2 J(J+1)}
$$

and must be set to zero when $J=0$ since no splitting occurs in this case.

The so-called normal Zeeman effect (Fig. 1.6) occurs in a transition where the Landé factors of the two levels are equal or if the energy levels have $J=0(M=0)$ and $J=1$ $(M=-1,0,1)$ respectively, meaning that the splitting results in exactly three different sublevels forming a Lorentz triplet. In a transition different to the particular case described above, the more general anomalous Zeeman effect occurs (Fig. 1.7), in which the splitting of an energy level results in more than three different sublevels and the Landé factors of the two transition levels are different. Thus, in the latter case, an effective Landé factor is defined as follows for a given transition (Shenstone and Blair 1929):

$$
g_{e f f}=\frac{1}{2}\left(g_{u}+g_{l}\right)+\frac{1}{4}\left(g_{u}-g_{l}\right)\left[J_{u}\left(J_{u}+1\right)-J_{l}\left(J_{l}+1\right)\right]
$$

where $l$ and $u$ refer to the lower and upper energy levels of the transition respectively. The effective Landé factor provides information on the magnetic sensitivity associated to the spectral line resulting from such transition.

In a quantum mechanical treatment, due to conservation of the angular momentum, the selection rule for electric-dipole transitions (which are the simplest kind of interaction between atoms and radiation field, see e.g., del Toro Iniesta 2003, Landi Degl'innocenti 
and Landolfi 2004) establishes that only those transitions that satisfy $\Delta M=M_{u}-M_{l}=$ $0, \pm 1$ are allowed, with the particular case of transitions from $M_{u}=0$ to $M_{l}=0$ being forbidden for $\Delta J=0$ (see Figs. 1.6 and 1.7 for an example of the allowed transitions in a normal and an anomalous Zeeman effect, respectively). Generally, transitions with $\Delta M=0$ are called $\pi$ components. Transitions with $\Delta M=-1$ correspond to red-shifted wavelengths with respect to the unperturbed line and are called $\sigma_{r}$ components, while transitions with $\Delta M=+1$ have blue-shifted wavelengths with respect to the unperturbed line and are called $\sigma_{b}$ components.

The resultant wavelengths associated to all possible transitions between the sublevels of two energy levels can be derived from equation 1.4. By considering the particular case of a transition that occurs in the visible or infrared part of the spectrum (where the Larmor frequency, which is the angular frequency of the circular motion of a charged particle under the presence of a uniform magnetic field, is much smaller than the frequency of the unperturbed line) between two levels that have each angular momentum quantum numbers given by $J_{u}$ and $J_{l}$ for the upper and lower energy levels respectively, and Landé factors $g_{u}$ and $g_{l}$ respectively, then the allowed transitions produce the splitting of the spectral lines into components with different wavelengths which are given by:

$$
\lambda_{M_{u} M_{l}}^{J_{u} J_{l}}=\lambda_{0}-\frac{\lambda_{0}^{2} e B}{4 \pi m_{e} c^{2}}\left(g_{u} M_{u}-g_{l} M_{l}\right),
$$

where $\lambda_{0}$ is the wavelength of the unperturbed line, and $M_{u}$ and $M_{l}$ are the magnetic quantum numbers of the upper and lower sublevels, respectively. Equation 1.7 implies that larger magnetic field strengths as well as larger wavelengths $\lambda_{0}$ produce larger line splittings and thus, due to the quadratic dependence of the wavelength separation on $\lambda_{0}$, the Zeeman effect is a handy diagnostic tool to infer the strength of photospheric magnetic fields particularly in the visible and infrared parts of the solar spectrum.

In practice, for the relatively small splittings seen outside sunspots, the splitting of a spectral line is generally calculated by considering the wavelength separation between the center of gravity of the $\sigma$ components (those that result from $\Delta M= \pm 1$ transitions), $\lambda_{\sigma}$, and the unperturbed wavelength, $\lambda_{0}$, as follows:

$$
\Delta \lambda=\left|\lambda_{0}-\lambda_{\sigma}\right|=\frac{\lambda_{0}^{2} e B}{4 \pi m_{e} c} g_{e f f},
$$

so that large effective Landé factors (implying high magnetic line sensitivities) make possible the measurement of relatively weak magnetic fields in the solar photosphere. However, the effects of weak photospheric magnetic fields on the spectral lines can be very similar to those produced by plasma motions (line broadening) when the wavelength separation in the line splitting is so small that the Zeeman components appear superimposed to each other. Hence, the identification of the nature of a spectral line broadening is, at first, not straightforward. However, velocity and temperature effects on the photons do not involve polarization, whilst a stream of photons emitted in the presence of a magnetic field show a net polarization (e.g., Herzberg 1945).

For the allowed transitions mentioned above, the so-called $\pi$ components correspond to linearly polarized photons in the direction of the magnetic field and thus, they can be observed only when the line-of-sight is perpendicular to $\vec{B}$. The so-called $\sigma_{r}$ components display right-handed circular polarization when observed in the direction of the magnetic 
field (for emission lines), while the $\sigma_{b}$ components have left-handed circular polarization when the line-of-sight is along the direction of the magnetic field. However, the polarization of the $\sigma$ components is generally elliptical for arbitrary directions, with the particular cases of being circular along the direction of $\vec{B}$ and linear in a direction perpendicular to $\vec{B}$ (Fig. 1.8), with their handedness mentioned above being reversed for a line-of-sight in the opposite direction to $\vec{B}$ and also for an absorption line. Thus, the analysis of the polarization of the light provides us information on the orientation of the magnetic field with respect to the observer.

\subsubsection{Solar polarimetry}

The polarization of the electromagnetic radiation is related to the geometrical aspects of the emission processes.

In the wave description of light, electromagnetic waves are transverse waves whose magnetic and electric fields, $\vec{B}$ and $\vec{E}$ respectively, oscillate perpendicularly (to each other and with respect to the direction of propagation, $\vec{n}$ ).

A wave equation can be derived directly from the set of Maxwell's equations for $\vec{E}$ (and analogously for $\vec{B}$ ) by considering a homogeneous isotropic medium with dielectric permitivity $\epsilon$ and magnetic permeability $\mu$, as follows:

$$
\nabla^{2} \vec{E}-\frac{\epsilon \mu}{c^{2}} \frac{d^{2} \vec{E}}{d t^{2}}=0
$$

In the following, we will define the polarization properties of electromagnetic waves in terms of the electric field oscillations by following the mathematical formulation presented by del Toro Iniesta (2003).

For our purposes and for most astrophysical applications, it is sufficient to consider the simplest solution to equation 1.9 which corresponds to plane waves, since the light sources of interest are located far enough from the observer. Such a solution, in a righthanded Cartesian system where the propagation direction $\vec{n}$ lies along the positive $z$-axis, describes the electromagnetic wave at a given point in space, $\vec{r}=(x, y, z)$, and at time $t$, as follows:

$$
\begin{aligned}
& E_{x}(t)=A_{x}(t) e^{-i\left(\omega t-\delta_{x}(t)\right)}, \\
& E_{y}(t)=A_{y}(t) e^{-i\left(\omega t-\delta_{y}(t)\right)}, \\
& E_{z}(t)=0 .
\end{aligned}
$$

Here $A_{j}(t)=a_{j}(t) e^{i \vec{k} \cdot \vec{r}}$ (with $j$ denoting the $x$ and $y$ components of vector $\vec{E}, a_{j}(t)$ being the real amplitude of each component, and $\vec{k}$ the so-called wave number vector) is the complex amplitude of the wave; $\omega=2 \pi v$ (with $v$ being the mean frequency of the wave) is the angular frequency, and $\delta_{j}(t)$ denotes the phases of the respective components.

Since the measurable quantities of these complex fields must be real, the polarization properties of an arbitrarily polarized electromagnetic wave can be described through the measurable set of Stokes parameters (Stokes 1852), which are real quantities defined as follows: 


$$
\begin{aligned}
& I=\left\langle a_{x}^{2}\right\rangle+\left\langle a_{y}^{2}\right\rangle, \\
& Q=\left\langle a_{x}^{2}\right\rangle-\left\langle a_{y}^{2}\right\rangle, \\
& U=2\left\langle a_{x} a_{y} \cos \delta(t)\right\rangle, \\
& V=2\left\langle a_{x} a_{y} \sin \delta(t)\right\rangle .
\end{aligned}
$$

Here the angular brackets denote time averages, and $\delta=\delta_{x}-\delta_{y}$ is the phase difference between the two orthogonal components of the electric field.

In equation 1.11, $I$ represents the total intensity of the light beam and therefore $I \geq 0$. $Q$ and $U$ provide a quantification of the net linear polarization of the electromagnetic wave, and $V$ of its net circular polarization.

Thus, given a set of four Stokes parameters, it is possible to determine the degree of polarization of the light, $p$, as follows:

$$
p=\frac{\sqrt{Q^{2}+U^{2}+V^{2}}}{I}
$$

with $0 \leq p \leq 1$.

Light is said to be natural or unpolarized when $p=0$, that is, when $Q=U=V=0$. Most of the light that is generated by thermal motions is unpolarized.

For totally or completely polarized light, $p=1$ and thus, the following relation is satisfied:

$$
I^{2}=Q^{2}+U^{2}+V^{2}
$$

while for partially polarized light:

$$
I^{2}>Q^{2}+U^{2}+V^{2}
$$

As mentioned above, the polarization state of an electromagnetic wave is given by the tip of the electric field in the $x-y$ plane. From equation 1.10 , it follows that this tip generally describes an ellipse:

$$
\frac{E_{x}^{2}}{a_{x}^{2}}+\frac{E_{y}^{2}}{a_{y}^{2}}-2 \frac{E_{x}}{a_{x}} \frac{E_{y}}{a_{y}} \cos \delta=\sin ^{2} \delta .
$$

Unlike in equation 1.10, here $E_{x}$ and $E_{y}$ stand for the real parts of both electric field components. The sign of $\sin \delta$ indicates the sense of rotation of the electric field (clockwise or right-handed when $\sin \delta>0$ and counter-clockwise or left-handed when $\sin \delta<0$ ). The phase difference between the two orthogonal components of the electric field, $\delta$, indicates the state of polarization as specified in Figure 1.9.

Typical devices designed to measure the polarization of the light detect the direction of motion of the electric field vector (linear analyzer-polarizer) as well as the phase difference, $\delta$, between $E_{x}$ and $E_{y}$ (linear retarder) separately (see, for example, Fig. 1.10). On the one hand, the linear retarder is a linear optical system that produces a phase delay in one of the Cartesian components of the electric field of the incident light (so-called slow axis) while the electric field along the other Cartesian component remains unchanged (socalled fast axis). If, for example, the electric field of the incident electromagnetic waves 

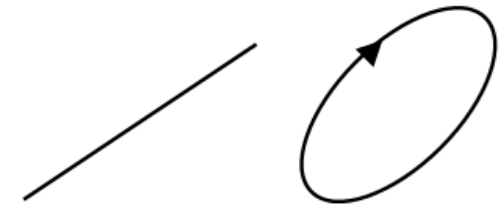

$\delta=0$

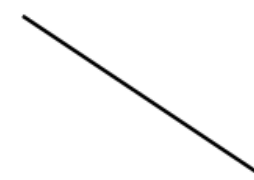

$0<\delta<\pi / 2$

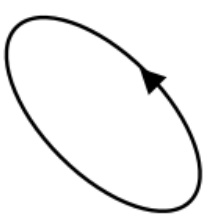

$\delta=\pi$
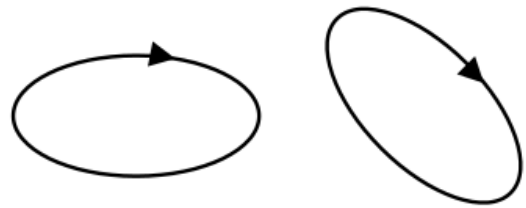

$\pi / 2<\delta<\pi$

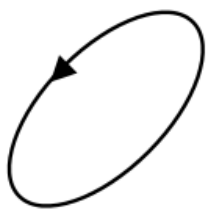

$\delta=3 \pi / 2$

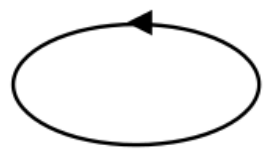

$3 \pi / 2<\delta<2 \pi$

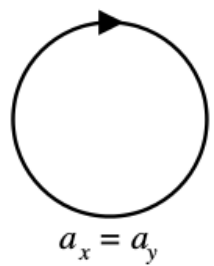

$\delta=\pi / 2$

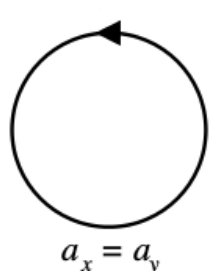

$\delta=3 \pi / 2$

Figure 1.9: Possible states of polarization of a totally polarized electromagnetic wave. In the most general case, the wave is elliptically polarized. Linear polarization occurs when either $E_{x}=0$ or $E_{y}=0$ or, otherwise, when either $\delta=0$ or $\delta=\pi$. Circular polarization occurs when the amplitude of both electric field components are equal, $a_{x}=a_{y}$, and either $\delta=\pi / 2$ or $\delta=3 \pi / 2$. Adapted from del Toro Iniesta (2003).

are given by $\vec{E}=E_{x} \hat{e_{x}}+E_{y} \hat{e}_{y}$ and the slow axis of the linear retarder is set in the $y$ direction, then the transmitted light beam will have electric field components $E_{x}^{\prime}=E_{x}$ and $E_{y}^{\prime}=E_{y} e^{i \delta}$ (Fig. 1.10, top panel). On the other hand, a linear analyzer-polarizer is a linear optical system acting on polarized light that allows the transmittance of all the incident light along its optical axis only. If the optical axis of a linear analyzerpolarizer is placed at an angle $\theta$ with respect to the $x$-axis, then the transmitted light will be characterized by $\vec{E}^{\prime}=E_{\theta} \hat{e_{\theta}}$, with $E_{\theta}=E_{x} \cos \theta+E_{y} \sin \theta$ (Fig. 1.10 , bottom panel). Thus, continuing with the same example, a basic polarimeter composed by a linear retarder that is followed by a linear polarizer-analyzer would transmit light whose electric field is given by $\vec{E}^{\prime}=\left[E_{x} \cos \theta+E_{y} \sin \theta e^{i \delta}\right] \hat{e_{\theta}}$. Furthermore, the output intensity, $I_{\text {out }}(\theta, \delta)=\left\langle E_{\theta}(\theta, \delta) E_{\theta}^{*}(\theta, \delta)\right\rangle$, is a linear combination of the four Stokes parameters as defined in Equations 1.11:

$$
I_{\text {out }}(\theta, \delta)=\frac{1}{2}(I+Q \cos 2 \theta+U \cos \delta \sin 2 \theta+V \sin \delta \sin 2 \theta) .
$$

The Stokes parameters can be determined by adjusting $\theta$ and $\delta$, given that:

$$
\begin{aligned}
& I=I_{\text {out }}(0,0)+I_{\text {out }}(\pi / 2,0), \\
& Q=I_{\text {out }}(0,0)-I_{\text {out }}(\pi / 2,0), \\
& U=I_{\text {out }}(\pi / 4,0)-I_{\text {out }}(3 \pi / 4,0), \\
& V=I_{\text {out }}(\pi / 4, \pi / 2)-I_{\text {out }}(3 \pi / 4, \pi / 2) .
\end{aligned}
$$

During the past decades, some more sophisticated devices have been designed in order to measure part or the full Stokes vector $\vec{I}=(I, Q, U, V)^{\top}$ simultaneously, for example, the Advanced Stokes Polarimeter (ASP, Lites et al. 1992, Tomczyk et al. 1992), the Fourier 


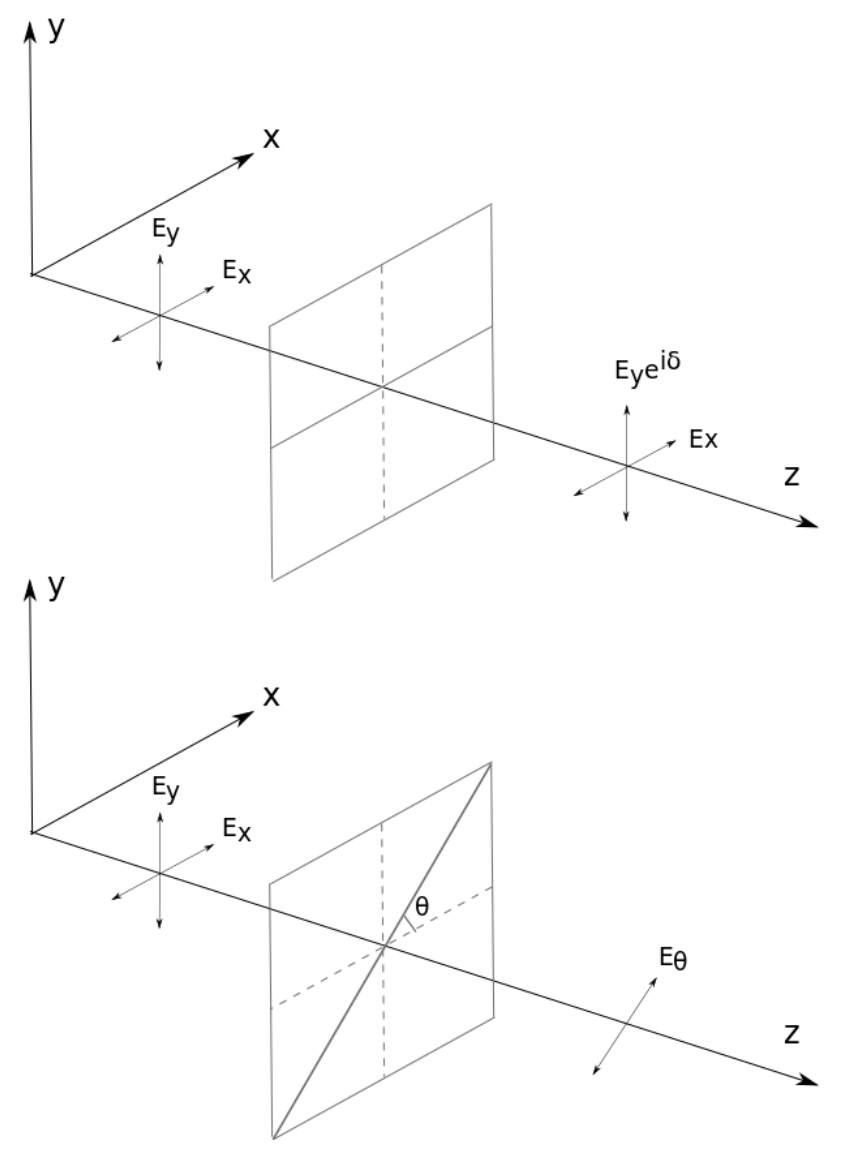

Figure 1.10: Example of a linear retarder (top) whose slow axis is placed along the Cartesian $y$-axis while the $x$-axis acts as the fast axis, and a linear analyzer-polarizer (bottom) whose optical axis is placed at an angle $\theta$ with respect to the $x$-axis.

Transform Spectrometer (FTS, Brault 1978) and the Zurich Imaging Polarimeter (ZIMPOL, Povel 1995, Gandorfer and Povel 1997). In those cases, the polarimeter comprises more than two components. The incident Stokes vector $\vec{I}_{\text {in }}$ is modified by the optical system in such a way that the output Stokes vector $\vec{I}_{\text {out }}$ is:

$$
\vec{I}_{\text {out }}=M \vec{I}_{\text {in }}
$$

where $M$ is the so-called Müller matrix of the whole optical system, which describes the transformation that all components of the optical system produce on the incident light. The Müller matrix $M$ is obtained by multiplying the Müller matrices of the individual optical components $M_{i}$ with each other, with $i$ being the $i$-th optical component encountered by the light. The multiplication of Müller matrices is not commutative so that each of them has to be placed in the proper order: $M=M_{n} \ldots M_{2} M_{1}$, assuming that the light encounters the optical devices from $M_{1}$ to $M_{n}$. In the example described above, in which a linear retarder is followed by a linear polarizer-analyzer having Müller matrices $M_{L R}$ and $M_{L P}$ respectively, the output Stokes vector of such a device would be given by: 


$$
\vec{I}_{\text {out }}=M_{L P} M_{L R} \vec{I}_{i n},
$$

where

$$
M_{L P}=\frac{1}{2}\left(\begin{array}{llll}
1 & 1 & 0 & 0 \\
1 & 1 & 0 & 0 \\
0 & 0 & 0 & 0 \\
0 & 0 & 0 & 0
\end{array}\right),
$$

and

$$
M_{L R}=\left(\begin{array}{cccc}
1 & 0 & 0 & 0 \\
0 & 1 & 0 & 0 \\
0 & 0 & \cos \delta & \sin \delta \\
0 & 0 & -\sin \delta & \cos \delta
\end{array}\right)
$$

for the particular case in which the linear polarizer-analyzer has its transmission direction along the $x$-axis, i.e. $\theta=0^{\circ}$, and the linear retarder has a retardance of $\delta$ and its fast axis is aligned with the $x$-axis.

In solar polarimetry, a spectropolarimetric observation can be represented as a fivedimensional hypercube that includes time, $t$, the Stokes parameters $(I, Q, U, V)$, the plane of the sky coordinates, $x$ and $y$, and the wavelength, $\lambda$ (Lagg et al. 2017). Given that the task is to determine the physical properties of the source medium as functions of space and time from the measurement of the Stokes parameters, i.e., from information regarding the intensity and the polarization state of the radiation as functions of the spatial position, spectral wavelength, and time (Lagg et al. 2017), the inverse problem has to be solved (see Section 1.3.5). To solve the inverse problem, many factors that limit the accuracy of the measurements, such as environmental as well as instrumental effects that modify the polarization state of the observed light, need to be taken into account.

Figure 1.11 shows an example of how the Stokes vector (normalized to the mean continuum intensity of the quiet Sun, $I_{C}$ ) is influenced by changes in strength and orientation of a magnetic field. The synthetic emergent Stokes profiles shown in the Figure were obtained under the Milne-Eddington approximation (see Section 1.3.5.1) by Borrero and Ichimoto (2011), assuming that the observer looks down along the $z$-axis. The magnetic field strength affects proportionally the splitting of the line. In particular, the field component along the observer's line-of-sight, $B_{L O S}$, affects proportionally the amplitude of Stokes $V$ in the case of weak fields (whose strengths are of the order of some hundred Gauss for typical lines in the visible part of the spectrum, but it depends on the Landé factor and the wavelength of the line), while for stronger fields, the amplitude of Stokes $V$ increases weakly but the separation between the red and the blue lobes increases proportionally with $B_{L O S}$. In contrast, the line effects due to field components that are transversal to the observer's line-of-sight, $B_{\perp}$, are observed only in the linear polarization profiles given by Stokes $Q, U$, and $I$. In this case, the magnetic field has been considered to be constant over all atmospheric heights. This assumption leads to symmetric Stokes $I$, $Q$, and $U$ profiles, and antisymmetric Stokes $V$ profiles with respect to the central wavelength. Nonetheless, the observed Stokes profiles frequently present large asymmetries that can be due to the existence of strong gradients in the magnetic field and/or in the velocity field. A discussion on how these cases are addressed is given in Section 1.3.5. 

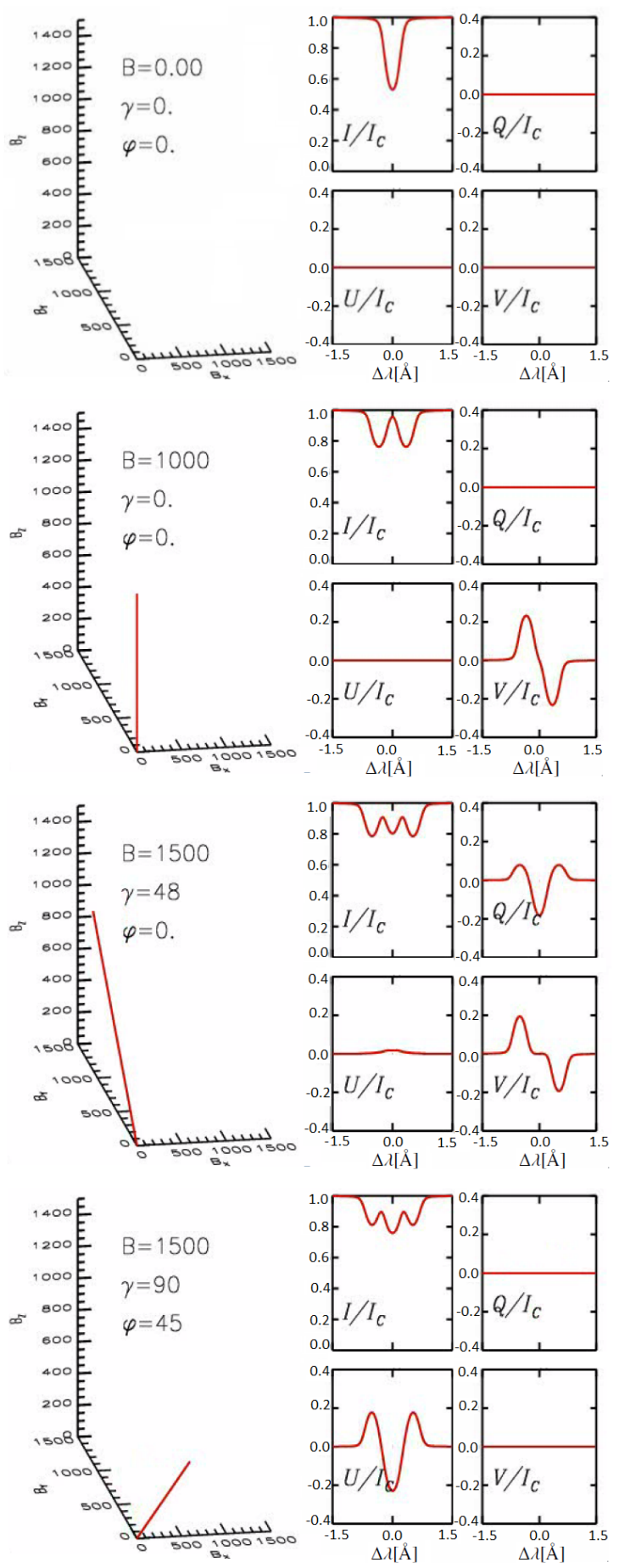

Figure 1.11: The influence of the strength and the orientation of the magnetic field on the four Stokes profiles of a spectral line, for an observer looking from the top (i.e., along the $z$-axis), obtained under the Milne-Eddington approximation (see Section 1.3.5.1). From top to bottom: (1) A spectral line formed in the absence of an external magnetic field; (2) A spectral line under the effects of a magnetic field, whose strength is $B=1000 \mathrm{G}$, inclination angle $\gamma=0^{\circ}$ and azimuth angle $\varphi=0^{\circ}$; (3) For $B=1500 \mathrm{G}, \gamma=48^{\circ}$ and $\varphi=0^{\circ}$; (4) For $B=1500 \mathrm{G}, \gamma=90^{\circ}$ and $\varphi=45^{\circ}$. Adapted from Borrero and Ichimoto (2011). 


\subsubsection{Hinode spectropolarimeter}

The Hinode spacecraft is an ongoing Japanese mission operated by the Institute of Space and Astronautical Science (ISAS), in collaboration with the Japanese Aerospace Exploration Agency (JAXA), the National Astronomical Observatory of Japan (NAOJ), the United Kingdom, and the United States (e.g., Kosugi et al. 2007). It is aimed to study the dynamics and the magnetism of the different layers in the solar atmosphere, in particular, the response of the solar corona to the magnetism of the photosphere and chromosphere.

The spacecraft was launched on 22 September 2006 with three telescopes on board: The X-ray telescope (XRT, e.g., Golub et al. 2007), the EUV Imaging Spectrometer (EIS, e.g., Culhane et al. 2006), and the Solar Optical Telescope (SOT, e.g., Tsuneta et al. 2008). The latter is a $50 \mathrm{~cm}$ Gregorian telescope designed to study photospheric magnetic fields using vector magnetic field and white light observations, and providing images with a spatial resolution of $0^{\prime \prime} .2-0^{\prime \prime} .3$. The SOT includes a Narrowband Filter Imager (NFI), a Broadband Filter Imager (BFI) and a Spectro-Polarimeter (SP).

The SP instrument (e.g., Lites et al. 2001, 2013) operates in a pair of absorption lines of Fe I, which are formed in the lower solar photosphere and whose central wavelengths are found (in the laboratory) at 630.15 and $630.25 \mathrm{~nm}$, each line having Landé factors $g_{\text {eff }}=1.67$ and 2.49, respectively. The SP instrument provides the line profiles in all four Stokes parameters with a spectral sampling of $2.15 \mathrm{pm}$ and a spatial sampling of $0^{\prime \prime} .16$, and it can operate in four different modes: normal mode, fast map mode, dynamics mode, and deep magnetogram mode (see Lites et al. 2013, for a detailed description of the SP instrument). The normal mode is nominally used to obtain scans of the vector magnetic field in active regions (AR).

The scan of the AR NOAA 10930, presented and analyzed in Chapter 3, was recorded by the SP instrument on 8 December 2006 while operating in normal mode using an exposure time of 4.8 seconds with a slit step of $0^{\prime \prime} .16$ in a $160^{\prime \prime} \times 164^{\prime \prime}$ map that was obtained in approximately 83 minutes. These observations are corrected for dark current, flat field, orbital drift and instrumental cross-talk by reducing the row data with IDL routines of the Solar-Soft package (Lites and Ichimoto 2013).

\subsubsection{Radiative transfer}

The four-dimensional Stokes vector, $\vec{I}=(I, Q, U, V)^{\top}$, is the measurable quantity providing all the information about the polarization state of the light.

However, one has to consider that the observed electromagnetic radiation first has to travel through the solar atmosphere before arriving to our instrument detectors and, given that the properties of the light are modified according to the properties of the medium, the determination of the original properties of the observed radiation is an intrinsically tricky task. Moreover, this problem is further complicated when considering the inhomogeneous characteristics of the solar atmosphere.

The radiative transfer theory studies the interaction of the radiation with the medium through which the light travels in order to consider the effects of this interaction on the properties of the observed radiation (Unno 1956, Rachkovsky 1962, Beckers 1969a,b, Wittman 1974, Landi Degl'innocenti 1983). The ultimate goal of solving this problem is the retrieval of the physical properties of the medium (such as the temperature, density, 
magnetic fields, and flow velocities) at a specific location on the Sun, at a given height (e.g., Solanki 1993, Frutiger 2000).

The first two physical phenomena to take into account regarding the interaction between the radiation and the medium are absorption and emission, which are, both, wavelength and optical-depth dependent. Thus, for $\alpha_{\lambda}$ and $j_{\lambda}$ being the absorption and the emission coefficients respectively, the change of the specific intensity $I_{\lambda}$ (which is a function of the position $\vec{r}$, time $t$, and the propagation direction $\vec{n}$ ) along the ray path, is given by the radiative transfer equation (RTE) as follows:

$$
\frac{d I_{\lambda}}{d z}=-\alpha_{\lambda} I_{\lambda}+j_{\lambda}
$$

which can also be written in terms of the source function, $S_{\lambda}=j_{\lambda} / \alpha_{\lambda}$, as follows:

$$
\frac{d I_{\lambda}}{d z}=-\alpha_{\lambda}\left(I_{\lambda}-S_{\lambda}\right)
$$

Under LTE conditions, the source function can be approximated by the Planck function, $B_{\lambda}(T)$, for the local temperature $T$, i.e.:

$$
S_{\lambda}=B_{\lambda}(T)=\frac{2 h c^{2}}{\lambda^{5}}\left[\frac{1}{e^{h c / k \lambda T}-1}\right] .
$$

For the simplified case of a non-emitting atmosphere, where $j_{\lambda}=0$, integration of equation 1.23 along the ray path (from $z_{0}$ to $z$ ) leads to:

$$
I_{\lambda}(z)=I_{\lambda}\left(z_{0}\right) e^{-\int_{z_{0}}^{z} \alpha_{\lambda}\left(z^{\prime}\right) d z^{\prime}}=I_{\lambda}\left(z_{0}\right) e^{-\tau_{\lambda}},
$$

where $\tau_{\lambda}$ is the optical depth. When $\tau_{\lambda} \gg 1$, the atmosphere is said to be opaque or optically thick, while, when $\tau_{\lambda} \ll 1$, the atmosphere is transparent or optically thin. Solution 1.25 describes an exponential decrease of the specific intensity with height when the atmosphere is non-emitting. However, stellar atmospheres generally have emission and absorption coefficients that are, both, non zero.

Another way to solve equation 1.23 analytically is to simplify the problem to a case in which $\alpha_{\lambda}$ does not depend on wavelength. This simplified model describes a gray atmosphere, which is nonetheless unreal. Moreover, only non-gray atmospheres can produce spectral lines when a temperature gradient exists. However, gray atmospheres are useful to examine some observable properties of stellar atmospheres, for example, they provide a first guess on the atmospheric temperature structure and basic radiative properties, all by using an exact solution (after further assumptions) of the RTE.

A complete treatment for the transport of light should include not only the changes of the specific intensity but also the changes in the polarization properties of the light. Hence, equation 1.23 should be applied to the complete Stokes vector, $\vec{I}$. Moreover, it should consider a realistic model atmosphere which includes, for example, the anisotropies produced by the presence of an external magnetic field. The form of the RTE, in its most general form, can be written as follows:

$$
\frac{d \vec{I}}{d z}=-\overrightarrow{K^{\prime}} \vec{I}+\vec{j}
$$


Here, $\vec{j}$ and $\overrightarrow{K^{\prime}}$ include all the physical and geometrical properties of the atmosphere and radiation system, with $\vec{j}$ being the total emission vector:

$$
\vec{j}=\kappa_{C}\left(S_{C} \overrightarrow{e_{0}}+S_{L} \vec{K} \overrightarrow{e_{0}}\right),
$$

where $\overrightarrow{e_{0}}=(1,0,0,0)^{\top}, \kappa_{C}$ is the continuum absorption coefficient, $S_{C}$ is the continuum source function, and $S_{L}$ is the source function inside the spectral line. In the simplest case, both source functions can be approximated as Planck functions under LTE conditions, with $S_{C}=B_{\lambda}\left(T_{C}\right)$ and $S_{L}=B_{\lambda}\left(T_{L}\right)$. If $T_{L}$ is a lower temperature than the continuum temperature, $T_{C}$, the superposition of both Planck functions results in an absorption line. In equation $1.26, \overrightarrow{K^{\prime}}$ is the total absorption matrix:

$$
\overrightarrow{K^{\prime}}=\kappa_{C}(\overrightarrow{1}+\vec{K})
$$

with $\overrightarrow{1}$ the unit matrix, and:

$$
\vec{K}=\left(\begin{array}{cccc}
\eta_{I} & \eta_{Q} & \eta_{U} & \eta_{V} \\
\eta_{Q} & \eta_{I} & \rho_{V} & -\rho_{U} \\
\eta_{U} & -\rho_{V} & \eta_{I} & \rho_{Q} \\
\eta_{V} & \rho_{U} & -\rho_{Q} & \eta_{I}
\end{array}\right)
$$

is the absorption matrix inside the spectral line or propagation matrix, whose elements are:

$$
\begin{aligned}
& \eta_{I}=1+\frac{\eta_{0}}{2}\left[\phi_{\pi} \sin ^{2} \gamma+\frac{\phi_{\sigma_{b}}+\phi_{\sigma_{r}}}{2}\left(1+\cos ^{2} \gamma\right)\right], \\
& \eta_{Q}=\frac{\eta_{0}}{2}\left[\phi_{\pi}-\frac{\phi_{\sigma_{b}}+\phi_{\sigma_{r}}}{2}\right] \sin ^{2} \gamma \cos 2 \varphi, \\
& \eta_{U}=\frac{\eta_{0}}{2}\left[\phi_{\pi}-\frac{\phi_{\sigma_{b}}+\phi_{\sigma_{r}}}{2}\right] \sin ^{2} \gamma \sin 2 \varphi, \\
& \eta_{V}=\frac{\eta_{0}}{2}\left[\phi_{\sigma_{r}}-\phi_{\sigma_{b}}\right] \cos \gamma, \\
& \rho_{Q}=\frac{\eta_{0}}{2}\left[\psi_{\pi}-\frac{\psi_{\sigma_{b}}+\psi_{\sigma_{r}}}{2}\right] \sin ^{2} \gamma \cos 2 \varphi, \\
& \rho_{U}=\frac{\eta_{0}}{2}\left[\psi_{\pi}-\frac{\psi_{\sigma_{b}}+\psi_{\sigma_{r}}}{2}\right] \sin ^{2} \gamma \sin 2 \varphi, \\
& \rho_{V}=\frac{\eta_{0}}{2}\left[\psi_{\sigma_{r}}-\psi_{\sigma_{b}}\right] \cos \gamma,
\end{aligned}
$$

Here, $\gamma$ and $\varphi$ represent the inclination and azimuthal angles of the magnetic field with respect to the line-of-sight (LOS), respectively; $\eta_{0}$ is the ratio between the continuum absorption coefficient and the line absorption coefficient; and, $\phi_{k}$ and $\psi_{k}$ are the absorption and dispersion profiles at the wavelength position of the $k=\pi, \sigma_{b}$ and $\sigma_{r}$ components of the Zeeman triplet, respectively, which follow the general expressions:

$$
\begin{aligned}
\phi_{k} & =\frac{1}{\sqrt{\pi}} \sum_{M} S_{M}^{k} H(u, a), \\
\psi_{k} & =\frac{1}{\sqrt{\pi}} \sum_{M} S_{M}^{k} F(u, a),
\end{aligned}
$$


with $S_{M}^{k}$ being proportional to the transition probability of each Zeeman component (recall that $\Delta M=-1,0,1$ for $\sigma_{r}, \pi, \sigma_{b}$ respectively) and quantifying the strength of each Zeeman component.

In equation 1.31, $H(u, a)$ and $F(u, a)$ are the Voigt and Faraday-Voigt functions, respectively, i.e.:

$$
\begin{aligned}
& H(u, a)=\frac{a}{\pi} \int_{-\infty}^{\infty} e^{-y^{2}} \frac{1}{(u-y)^{2}+a^{2}} d y, \\
& F(u, a)=\frac{1}{\pi} \int_{-\infty}^{\infty} e^{-y^{2}} \frac{v-y}{(u-y)^{2}+a^{2}} d y,
\end{aligned}
$$

where $a$ is called the damping parameter, and $u=u_{0}+u_{L O S}+u_{B, M}^{k}$, with:

$$
\begin{aligned}
& u_{0}=\frac{\lambda-\lambda_{0}}{\Delta \lambda_{D}}, \\
& u_{L O S}=\frac{\lambda_{0} v_{L O S}}{c \Delta \lambda_{D}}, \\
& u_{B, M}^{k}=-\frac{\Delta \lambda_{B, M}^{k}}{\Delta \lambda_{D}},
\end{aligned}
$$

where $v_{L O S}$ is the plasma bulk velocity along the LOS and $\Delta \lambda_{B, M}^{k}$ (which follows directly from equation 1.8) is the wavelength shift of each Zeeman component due to the Zeeman splitting in the presence of an external magnetic field, $B$.

The general solution of equation 1.26, in terms of the source function vector $\vec{S}=$ $\left(B_{\lambda}(T), 0,0,0\right)^{\top}$ and the propagation matrix $\vec{K}$, and by using the continuum optical depth, $\tau_{c}$ (with $d \tau_{c}=\vec{K} d z$ ), as the independent variable, can be found by integrating between different optical depth levels, $\tau_{0}$ and $\tau_{1}$ :

$$
\vec{I}\left(\tau_{1}\right)=\vec{O}\left(\tau_{1}, \tau_{0}\right) \vec{I}\left(\tau_{0}\right)-\int_{\tau_{0}}^{\tau_{1}} \vec{O}\left(\tau_{1}, \tau_{c}\right) \vec{K}\left(\tau_{c}\right) \vec{S}\left(\tau_{c}\right) d \tau_{c}
$$

where $\vec{O}$ is called the evolution operator. This solution, in a semi-infinite stellar atmosphere (where $\tau_{1}=0$ and $\tau_{0} \rightarrow \infty$ ) leads to:

$$
\vec{I}(0)=\int_{0}^{\infty} \vec{O}\left(0, \tau_{c}\right) \vec{K}\left(\tau_{c}\right) \vec{S}\left(\tau_{c}\right) d \tau_{c}
$$

where the evolution operator:

$$
\vec{O}\left(0, \tau_{c}\right)=e^{-\int_{0}^{\tau_{c}} \vec{K}\left(\tau_{c}\right) d \tau_{c}}
$$

has no analytical solution in the general case.

Various numerical codes have been developed in the last decades in order to solve the RTE in its general form. Some of the most popular in the solar physics community are, for example, the SIR code (Ruiz Cobo and del Toro Iniesta 1992, Bellot Rubio 1998), the SPINOR code based on the STOPRO routines (Solanki 1987, Frutiger 2000, van Noort 2012, van Noort et al. 2013), the LILIA code (Socas-Navarro 2001), among others. However, to solve the RTE, the codes need to know the stratification of the physical properties in the atmosphere (input) in order to obtain the resultant Stokes profiles (output). These are generally called synthetic Stokes profiles, $\overrightarrow{I_{s y n}}$. 


\subsubsection{Inversions}

The SOT/SP instrument on board the Hinode spacecraft provides the full-Stokes dataset in a certain region of the solar surface. We have seen that the RTE for polarized light describes the formation of the Stokes profiles when the physical properties of the medium are known, which means that the inverse problem needs to be solved in solar physics since the fundamental problem is to infer the physical properties of the solar atmosphere (output) from a given set of observed Stokes profiles, $\overrightarrow{I_{o b s}}$ (input).

The typical procedure to solve the inverse problem is called inversion and consists in, first, defining the stratification (in optical depth ${ }^{1}, \tau$ ) of a model atmosphere:

$$
M(\tau)=\left[T(\tau), B(\tau), v_{L O S}(\tau), \gamma_{L O S}(\tau), \varphi(\tau), v_{M I C}(\tau), v_{M A C}(\tau), p_{e}(\tau)\right]
$$

where $T$ is the temperature, $B$ is the magnetic field strength, $v_{L O S}$ is the LOS velocity, $\gamma_{L O S}$ is the magnetic field inclination angle with respect to the $\operatorname{LOS}$ direction, $\varphi$ is the magnetic field azimuthal angle, $v_{M I C}$ is the microturbulent velocity, $v_{M A C}$ is the macroturbulent velocity and $p_{e}$ is the electron pressure.

By providing a first guess of the atmospheric physical parameters in equation 1.37 and solving the RTE, it is possible to obtain a set of synthetic Stokes profiles, $\overrightarrow{I_{s y n}}$, which are afterwards compared to the set of observed Stokes profiles, $\overrightarrow{I_{o b s}}$. In this way, a merit function can be defined as:

$$
\chi^{2}=\sum_{i=1}^{4} \sum_{j=1}^{N}\left[\frac{I_{s y n, i}\left(\lambda_{j}\right)-I_{o b s, i}\left(\lambda_{j}\right)}{\sigma_{i, j}}\right]^{2},
$$

where $i$ stands for each of the Stokes parameter in the full Stokes vector, $j$ indicates the wavelength position and $\sigma_{i, j}$ represents the expected uncertainties in the observed profiles. Inversions work by minimizing the merit function in an iterative way through a systematic modification of the atmospheric parameters (for example using the Marquardt algorithm, see e.g., Press et al. 2007). The $\vec{I}_{\text {syn }}$ resulting from the minimization procedure is called 'best-fit' to the observed Stokes profiles, and the set of physical atmospheric parameters producing such 'best-fit' is assumed to correspond to that present in the observed region of the solar atmosphere. This procedure is usually applied pixel by pixel. The merit function, as defined in equation 1.38, can be very complex and present various local minima, requiring to ensure that inversion codes find an absolute minimum and, therefore, provide a stable solution.

Furthermore, after the inversion, it is necessary to take into account the inherent $180^{\circ}$ ambiguity of the magnetic field azimuthal angle, $\varphi$. This ambiguity is an intrinsic problem due to the linear polarization of the Zeeman effect (Harvey 1969) which causes that $\varphi$ and $\varphi+180^{\circ}$ produce indistinguishable Stokes profiles. To solve this problem, several algorithms have been developed (see e.g., Metcalf et al. 2006, for an overview). In Chapter 3, the Non-Potential Field Computation method (NPFC, Georgoulis 2005) is employed to solve the ambiguity problem after the inversion of the Stokes profiles observed with the Hinode SOT/SP instrument in AR NOAA 10930.

\footnotetext{
${ }^{1}$ For reasons of comparability and convention, we use the optical depth at $500 \mathrm{~nm}, \tau_{500}$.
} 


\subsubsection{The Milne-Eddington approximation}

The Milne-Eddington (ME) approximation provides a simple description of spectral line formation by assuming that all the physical quantities relevant to line formation in the model atmosphere are constant with depth (e.g., Unno 1956, Rachkovsky 1962), with the parameters of the Voigt and Faraday-Voigt functions governing the shape of the Stokes profiles of unsaturated or only weakly saturated lines.

In this approximation, the source function, $\vec{S}=\vec{S}_{0}+\vec{S}_{1} \tau_{c}$, varies linearly with optical depth and the propagation matrix, $\vec{K}\left(\tau_{c}\right)=\vec{K}_{0}$, is assumed to be constant. Therefore, the evolution operator is:

$$
\vec{O}\left(0, \tau_{c}\right)=e^{-\vec{K}_{0} \tau_{c}}
$$

and, hence, the RTE has an analytical solution given by:

$$
\vec{I}(0)=\int_{0}^{\infty} e^{-\vec{K}_{0} \tau_{c}} \vec{K}_{0}\left(\vec{S}_{0}+\vec{S}_{1} \tau_{c}\right) d \tau_{c}=\vec{S}_{0}+\vec{K}_{0}^{-1} \vec{S}_{1}
$$

which leads to an explicit form of the Stokes vector, known as the Unno-Rachkovsky solution:

$$
\begin{aligned}
& I(0)=S_{0}+\frac{S_{1} \eta_{1}}{\Delta} \eta_{I}\left(\eta_{I}^{2}+\rho_{Q}^{2}+\rho_{U}^{2}+\rho_{V}^{2}\right), \\
& Q(0)=\frac{S_{1}}{\Delta}\left[\eta_{I}^{2} \eta_{Q}+\eta_{I}\left(\eta_{V} \rho_{U}-\eta_{U} \rho_{V}\right)+\rho_{Q}\left(\eta_{Q} \rho_{Q}+\eta_{U} \rho_{U}+\eta_{V} \rho_{V}\right)\right], \\
& U(0)=\frac{S_{1}}{\Delta}\left[\eta_{I}^{2} \eta_{U}+\eta_{I}\left(\eta_{Q} \rho_{V}-\eta_{V} \rho_{Q}\right)+\rho_{U}\left(\eta_{Q} \rho_{Q}+\eta_{U} \rho_{U}+\eta_{V} \rho_{V}\right)\right], \\
& V(0)=\frac{S_{1}}{\Delta}\left[\eta_{I}^{2} \eta_{V}+\eta_{I}\left(\eta_{U} \rho_{Q}-\eta_{Q} \rho_{U}\right)+\rho_{V}\left(\eta_{Q} \rho_{Q}+\eta_{U} \rho_{U}+\eta_{V} \rho_{V}\right)\right],
\end{aligned}
$$

with $\Delta$ being the determinant of the propagation matrix:

$$
\Delta=\eta_{I}^{2}\left(\eta_{I}^{2}-\eta_{Q}^{2}-\eta_{U}^{2}-\eta_{V}^{2}+\rho_{Q}^{2}+\rho_{U}^{2}+\rho_{V}^{2}\right)-\left(\eta_{Q} \rho_{Q}+\eta_{U} \rho_{U}+\eta_{V} \rho_{V}\right)^{2}
$$

This model is in many cases too simplistic, particularly when dealing with a highly stratified atmosphere whose physical parameters display large variations with optical depth. However, the ME approximation is generally a useful tool to understand the behavior of the spectral lines formed in the solar atmosphere and can predict the observable phenomenon known as limb-darkening or center-to-limb variation of the average intensity.

There are various inversion codes based on ME model atmospheres, for example, the Milne-Eddington gRid Linear Inversion Network (MERLIN) code designed at the High Altitude Observatory (HAO) in USA, the Very Fast Inversion of the Stokes Vector (VFISV, Borrero et al. 2011), the HAO's Extended Inversion Code (HEXIC) based on VFISV, the Helium Line Information Extractor code (HeLIx ${ }^{+}$, Lagg et al. 2004, 2009), etc.

In particular, the $\mathrm{HeLIx}{ }^{+}$code was designed to analyze the chromospheric He I triplet at $1083 \mathrm{~nm}$, but can also be applied in one or multiple photospheric spectral lines to retrieve the vector magnetic fields and flow velocities, by using single- or multi-component 
model atmospheres. The code uses the Voigt functions to describe the Zeeman components of the spectral lines, and the considered model atmosphere includes 8 free parameters: the magnetic field strength, $B$, the LOS velocity, $v_{L O S}$, the inclination and azimuth angles of the magnetic field vector, $\gamma_{L O S}$ and $\varphi$ respectively, the Doppler width, $\Delta \lambda_{D}$, the damping constant, $a$, the gradient of the source function, $S_{1}$, and the opacity ratio between line-center and continuum, $\eta_{0}$ (see Lagg et al. 2004, 2009, for more a detailed description of the HeLIx ${ }^{+}$code).

\subsubsection{SPINOR inversions}

SPINOR (Stokes-Profiles-INversion-O-Routines; Frutiger 2000, Frutiger et al. 2000) is an inversion code developed at the ETH Zürich and at the Max Planck Institut für Aeronomie.

These inversions are based either on single- or on multi-component plane-parallel model atmospheres whose physical quantities only depend on the optical depth. Both modalities are applicable to all positions on the solar disk $(0<\mu=\cos \theta \leq 1$, where $\theta$ is the heliocentric angle). The single-component atmospheres are ideally applicable to cases in which the atmospheric feature of interest is spatially resolved, given that they provide a description of the spatially averaged resolution element. By contrast, the multicomponent atmospheres are useful when the atmospheric feature of interest is spatially unresolved. In the latter case, SPINOR can associate a plane-parallel atmosphere to each of the unresolved surface fractions within the resolution element and thus, the total emergent Stokes profiles are obtained as the weighted sum over the emergent spectra from each component (Frutiger 2000). Furthermore, SPINOR can also be used on flux-tube model atmospheres (Frutiger and Solanki 2001), on discontinuous atmospheres along the LOS or interlaced models (Borrero et al. 2006), and even to obtain disk-integrated spectra (Frutiger et al. 2005).

In general, for each atmospheric component, the code models the stratification of all quantities as functions of the logarithmic optical depth $(\log \tau)$ by using a spline approximation between a selected number of optical depth nodes and linearly extrapolating downwards to $\log \tau>1.4$ and upwards to a given optical depth if required. Moreover, SPINOR does not require that the selected nodes are equally-spaced along the atmospheric grid, but it allows the user to choose their location, for example, at those optical depths where the spectral line is more sensitive.

The gas and electron pressures are derived from the temperature stratification by assuming hydrostatic equilibrium and LTE (cf. Gustafsson 1973), while the macroturbulent velocity is assumed to be depth independent. In addition to the micro- and macro-turbulence velocities, which account for the line broadening due to velocity fields below the resolution element, other broadening mechanisms are also considered, such as radiative broadening and broadening by collisions with neutrals.

The SPINOR inversion code includes the following modules: the STOPRO routines (Solanki 1987, Solanki et al. 1992a) to solve the RTE under the assumption of LTE, and the INVERT package to find the best-fit to the observed spectra by minimizing the merit function using the Levenberg-Marquardt algorithm (Press et al. 1992). Finally, the synthesis of the Stokes profiles can be performed either by using the Diagonal Element Lambda Operator (DELO, Rees et al. 1989) or the Hermitian method (Bellot Rubio et al. 
1998), by taking into account Rayleigh and Thompson scattering.

\subsubsection{SPINOR 2D inversions}

The spatially coupled version of the SPINOR inversion code (SPINOR 2D, van Noort 2012, van Noort et al. 2013) uses a sophisticated technique to take into account the image degradation effects produced by the point-spread function (PSF) of the telescope and other instrumental effects in order to minimize the resulting errors while increasing the spatial resolution.

The removal process of the image degradation is applied to the synthetic data rather than by deconvolving the data themselves. Thus, the SPINOR 2D code calculates the best-fit to the full Stokes spectra by performing a coupled inversion of all the pixels simultaneously in order to self-consistently take into account the effects produced by the stray-light from the neighboring pixels.

Furthermore, the SPINOR 2D inversion code considers a spatial grid that is denser than that in the original data by employing a single-component atmospheric model per pixel, whose physical parameters are initially defined over a certain number of optical depth positions, which are then interpolated using a bi-cubic spline approximation. The stratifications are then extrapolated linearly above the selected optical depth top node up to a user defined threshold (typically $\log \tau=-4$ ), and downwards (below $\log \tau=0$ ) up to $\sim \log \tau=1.3$.

The inverted atmospheric parameters returned by the code correspond to the best fits to the Stokes profiles once the blurring effect of the telescope diffraction pattern has been taken into account. Therefore, the inverted parameters display significantly improved spatial resolutions which allows structures at the diffraction limit of the telescope to be properly resolved.

The SPINOR 2D inversions presented in this work (Chapters 3 and 5) use a pixel size of $0^{\prime \prime} .08$, which is a factor 2 smaller than in the original data from Hinode SOT/SP, which have a pixel size of $0^{\prime \prime} .16$. Also, all the atmospheric free parameters are allowed to vary at three optical depth nodes ${ }^{2}$, placed at $\log \tau=-2.0,-0.8$ and 0 (see also Siu-Tapia et al. 2017).

\subsection{Magnetohydrodynamical simulations}

So far, I have introduced some basic concepts, tools and analysis techniques that are useful to study the solar photosphere observationally. We have seen that all the information that a solar observer can obtain is due to the incident electromagnetic radiation, most of which belongs to the wavelength range $200-1600 \mathrm{~nm}$ (Fig. 1.4) and originates in a relatively thin layer of the solar atmosphere below which the medium is completely opaque. The observational study of the lower solar atmosphere as well as our conception of sub-photospheric layers as retrieved by indirect techniques within the scope of helioseismology, can both be supplemented by theoretical and numerical approaches.

\footnotetext{
${ }^{2}$ Computations of the response functions (which basically describe the sensitivity of the lines to small changes in the atmospheric quantities) for the pair of Fe I lines at 630.15 and $630.25 \mathrm{~nm}$, suggest that they are mainly formed within the $\log \tau_{500}=[-3,0]$ range, which corresponds to a formation height range of about $400 \mathrm{~km}$ by considering hydrostatic equilibrium (e.g., del Toro Iniesta 2003, Riethmüller 2013).
} 
Magnetohydrodynamics (MHD) is a useful approximation to study the macro-physics of the solar interior and its atmosphere. In particular, MHD has been a valuable tool for studying the interaction between convective flows and magnetic fields.

In general, MHD describes the physics of well-conducting fluids or plasmas that are quasi-neutral and whose particles display a collective behavior. It assumes that there is no charge separation within the scales of interest and that the characteristic velocities have much smaller speeds than the speed of light, so that the displacement current is neglected in the Maxwell equations. Furthermore, the considered time- and length-scales are large compared to the characteristic scales in the plasma, such as those associated with the plasma frequency $\omega_{p}$ and the Debye length $\lambda_{D}$ respectively, which are indeed very small in the solar photosphere $\left(\omega_{p}^{-1} \sim 10^{-12} \mathrm{~s}\right.$ and $\left.\lambda_{D} \sim 10^{-7} \mathrm{~m}\right)$ compared to the temporal and spatial resolutions considered in MHD simulations and with the typical sizes of observed features.

\subsubsection{The MURaM code}

The MURaM (MPS/University of Chicago Radiative MHD) code (Vögler 2003, 2004, Vögler et al. 2004, 2005) is a three-dimensional (3D) radiative MHD code designed for applications in the solar convection zone and photosphere through realistic numerical simulations. It includes non-local and non-gray radiative transfer, field-aligned heat conduction and takes into account partial ionization effects.

The code solves the non-ideal MHD equations (derivations can be found in, e.g., Priest 1982, Cap 1994, Choudhuri 1998) in a 3D Cartesian space assuming constant gravitational acceleration, $\vec{g}$. All the spatial derivatives are discretized as fourth-order centered differences and the time stepping is explicit (fourth-order Runge-Kutta solver) (Vögler 2003).

Thus, the system of MHD equations solved by the code, in terms of density, $\rho$, momentum density, $\rho \vec{v}$, total energy per volume, $e$, and magnetic field strength, $B$, are:

$$
\begin{gathered}
\frac{\partial \rho}{\partial t}+\nabla \cdot(\rho \vec{v})=0, \\
\rho\left(\frac{\partial \vec{v}}{\partial t}+(\vec{v} \cdot \nabla) \vec{v}\right)=-\nabla p+\frac{1}{4 \pi}(\nabla \times \vec{B}) \times \vec{B}+\rho \vec{g}+\nabla \cdot \vec{\tau}, \\
\frac{\partial \vec{B}}{\partial t}=\nabla \times(\vec{v} \times \vec{B})+\eta \nabla^{2} \vec{B}, \\
\nabla \cdot \vec{B}=0, \\
\frac{\partial e}{\partial t}+\nabla \cdot\left[\left(e+p+\frac{B^{2}}{8 \pi}\right) \vec{v}-\frac{(\vec{v} \cdot \vec{B})}{4 \pi} \vec{B}\right]= \\
\frac{1}{4 \pi} \nabla \cdot(\vec{B} \times \eta \nabla \times \vec{B})+\nabla \cdot(\vec{v} \cdot \vec{\tau})+\nabla \cdot(K \nabla T)+\rho(\vec{g} \cdot \vec{v})+Q_{\mathrm{rad}},
\end{gathered}
$$


where $\vec{v}$ is the flow velocity, $p$ is the gas pressure, $\eta$ is the magnetic diffusivity, $T$ is the temperature, $K$ is the thermal conductivity, $\vec{\tau}$ is the viscous stress tensor, and $Q_{\text {rad }}$ is the source term that accounts for radiative processes, such as radiative heating and cooling.

Equation 1.43 is the continuity equation representing mass conservation, equation 1.44 is the equation of motion, equation 1.45 is the induction equation, equation 1.46 is the divergence-free condition for the magnetic field, and equation 1.47 is the energy equation which accounts for the total energy density per volume $e$, i.e. the sum of internal, kinetic and magnetic energy densities as follows (in cgs units):

$$
e=e_{i n t}+\frac{\rho|\vec{v}|^{2}}{2}+\frac{|\vec{B}|^{2}}{8 \pi}
$$

In equation 1.45 , the diffusive term $\eta \nabla^{2} \vec{B}$ is neglected and the diffusion of the magnetic field is described by an artificial (numerical) diffusivity. In equation 1.47, the source term for conductive heat transport $\nabla \cdot(K \nabla T)$ is negligible for the solar photosphere. Furthermore, in the MURaM approximations of both the energy equation and the equation of motion, the viscous dissipation is also neglected and described artificially. The artificial diffusivities for all physical quantities depend on the resolution and are calculated in each time step for each coordinate direction (Vögler 2003).

The source term for radiative heating and cooling, $Q_{\text {rad }}$, is calculated by solving the RTE written as follows:

$$
\frac{d I_{\lambda}}{d \tau}=\rho \kappa_{\lambda}\left(I_{\lambda}-S_{\lambda}\right),
$$

where $I_{\lambda}$ is the specific intensity, $S_{\lambda}$ the source function (Planck function assuming LTE conditions) and $\kappa_{\lambda}$ the opacity at a given wavelength. Therefore, $Q_{\text {rad }}$ is calculated as an integration over solid angle and wavelength:

$$
Q_{\text {rad }}=\int_{\lambda} \int_{\Omega} \rho \kappa_{\lambda}\left(I_{\lambda}-S_{\lambda}\right) d \Omega d_{\lambda}
$$

The system of MHD equations 1.43-1.47 is closed by the equation of state to describe the relations between the thermodynamical quantities of the fluid, $T$ and $p$, as functions of $\rho$ and $e_{\text {int }}$ :

$$
\begin{aligned}
& T=T\left(e_{i n t}, \rho\right), \\
& p=p\left(e_{i n t}, \rho\right),
\end{aligned}
$$

for a partially ionized plasma which does not obey the ideal gas law, as occurs in the solar near-surface layers. The code takes into account the first ionization of the 11 most abundant elements, and equations 1.49 are approximated based on the OPAL equation of state (Rogers 1994, Rogers et al. 1996) and the solar composition given by Anders and Grevesse (1989).

The size of the simulation domain and the grid-cell size, as well as the initial and boundary conditions on the magnetic field, depend on the phenomenon of interest. The upper boundary of the computational domain can be set as either closed or open, while the bottom boundary is left open, i.e., the material is allowed to freely enter or leave the computational domain. A closed boundary constrains the vertical convective fluxes of 
mass, energy, and horizontal momentum by forcing them to vanish at the top boundary. This can lead to unphysical effects such as the reflection of waves and shocks. However, by placing the upper boundary near or above the traditional temperature minimum at the top of the photosphere, where the plasma density is very low, it is possible to reduce its influence on the layers of main interest for this thesis (lower half of the photosphere and solar interior).

In this thesis (Chapter 4), the MURaM code is used to study magneto-convection and the nature of large-scale flows occurring in sunspot penumbrae by means of a 3D high-resolution sunspot simulation setup made by Matthias Rempel ${ }^{3}$. The employed simulations were first described and reported in Rempel (2015).

\footnotetext{
${ }^{3} \mathrm{HAO}, \mathrm{NCAR}, \mathrm{USA}$
} 


\section{Sunspots: Review}

Sunspots are, by far, the most striking features observed on the solar surface and their existence has been known for more than 2000 years. They are seen as dark regions in the photosphere, a consequence of large magnetic field concentrations that interact with the solar plasma and inhibit the flow of heat from the deeper layers. Even though sunspots have been widely studied for almost 400 years, both individually and collectively, there are many aspects regarding their structure and evolution that are still not fully understood.

On the one hand, understanding the collective behavior of sunspots is important for building a comprehensive solar dynamo model. This collective behavior includes all those aspects summarized in the butterfly diagrams (e.g., the 11-year period of the sunspot cycle, the tendency of sunspots to appear at well-defined latitudes and their equatorward drift in the course of the solar cycle, the 22-year magnetic cycle, Hale's polarity law, and the observed tilt of sunspot pairs or Joy's law; see e.g. reviews by Solanki 2003, Hathaway 2015). On the other hand, understanding the global properties of sunspots (e.g., their mean sizes and lifetimes, their 3D magnetic field configuration, their brightness, dynamics and evolution, etc.) and their fine-scale structure, is important for gaining insight on how the energy is transported within sunspots, how magnetic fields are transported through the convection zone and finally emerge through the surface, and also, to better understand how they interact with the convective material at different scales.

Although the subsurface structure of sunspots is poorly known and its study relies mostly on numerical modeling, the recent advances achieved in solar instrumentation (in terms of spatial resolution) allow us to study in great detail the superficial fine structure of sunspot umbrae and penumbrae. In this chapter, I will present a brief overview of our present understanding of sunspot structure and, in particular, of the penumbral fine structure and its characteristic outward flow of material discovered by John Evershed more than 100 years ago (Evershed 1909) which is the central subject of this thesis.

\subsection{Sunspot formation and decay}

Sunspots belong to active regions, and thus the emergent magnetic flux responsible for the formation of a sunspot is also related to the formation of the whole active region.

It has been suggested that active regions form from large $\Omega$-shaped, deeply rooted magnetic flux tubes that originate near the tachocline and which rise buoyantly through the convection zone to the photosphere in time scales that depend on the field strength in the flux tube (e.g. Zwaan 1992, Harvey 1993, Parker 1994, Strous 1994, Solanki 2003).

The top of the flux tubes would continue rising through the solar surface, and the strong convection near the top of the convection zone would shred the large flux tube into 


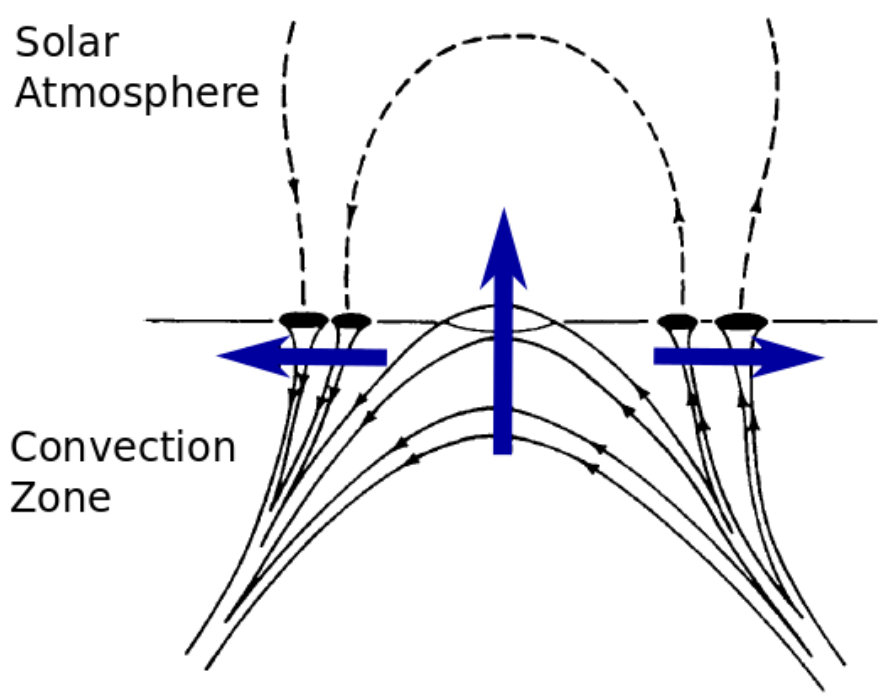

Figure 2.1: Sketch of an emerging flux tube producing two pairs of spots with opposite field polarities. The black arrows indicate the direction of the field and the blue arrows the motion of the flux tubes. A pressure balance between the magnetic and the hydrostatic pressures would cause the density inside the flux tube to be lower than in its surroundings due to the stronger magnetic pressures inside the flux tube. Therefore, the magnetic flux tube becomes buoyant. Adapted from Schrijver and Zwaan (2000).

many smaller flux tubes. This process would result in the emergence of a pair of magnetic field patches of opposite polarity, which move away from each other and are still magnetically connected in the atmosphere above, as shown in the sketch in Figure 2.1 (Schrijver and Zwaan 2000). Such small flux elements are advected by the supergranular convection and can eventually coalesce to form pores and later grow and further coalesce to form sunspots (e.g., Vrabec 1971, 1974, McIntosh 1981, Harvey and Harvey 1973, Zwaan 1985, Keppens and Martínez Pillet 1996, Leka and Skumanich 1998, Schlichenmaier 2009). However, this rising flux-tube model for the formation of sunspots has recently been questioned. There is observational evidence that stars without a tachocline show an activity cycle, a fact that cannot be explained by this model. In addition, state-of-the-art MHD simulations point to structure formation in the top layers of the convection zone not requiring the winding up of flux tubes at the tachocline (e.g., Brandenburg et al. 2016).

Sunspots have formation time-scales between hours and days, whereas their penumbrae (including the establishment of both the characteristic penumbral magnetic field configuration and the Evershed flow) form in time-scales from minutes to hours (Leka and Skumanich 1998, Yang et al. 2003, Schlichenmaier et al. 2010b, 2012, Romano et al. 2014, Murabito et al. 2016). Soon after the formation of a sunspot, its decay phase starts, i.e. it starts loosing magnetic flux (e.g., McIntosh 1981). However, new magnetic flux can also emerge after the formation phase (e.g., Solanki 2003). Sunspot lifetimes can span weeks or months. These time-scales are much shorter than the magnetic diffusion time-scales, which are of the order of $10^{2}-10^{3}$ years (e.g., Priest and Forbes 2000, Moradi et al. 2010). Therefore, additional mechanisms that can accelerate sunspot decay processes have been considered, such as convective instabilities, the fluting instability, 

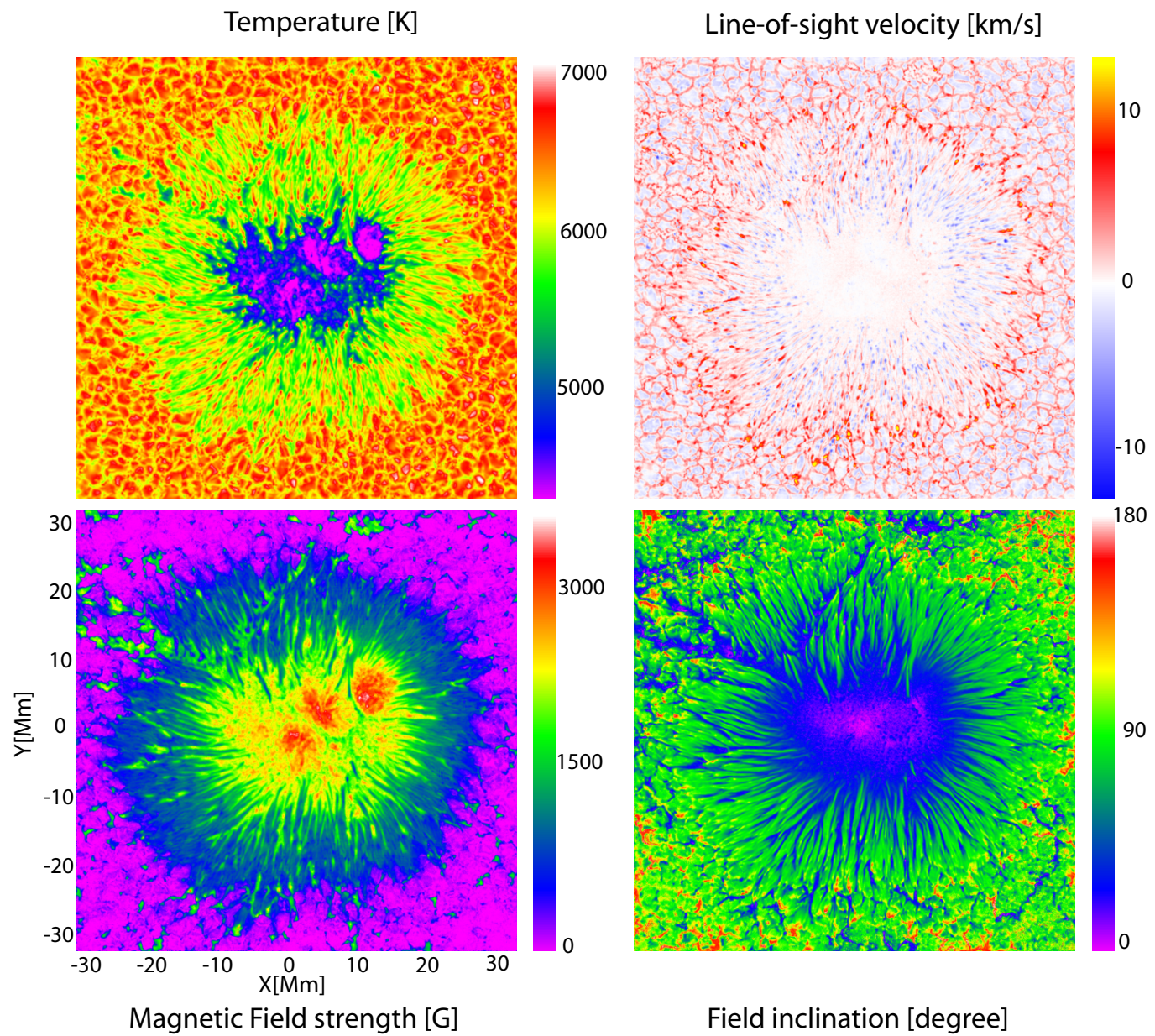

Figure 2.2: Maps of physical parameters in a sunspot (AR NOAA 10933) observed near the solar disk center $(\mu \approx 1)$ by the SOT/SP instrument on board Hinode. Clockwise: Temperature, LOS velocity $v_{L O S}$, field inclination angle $\gamma_{L O S}$, and magnetic field strength at the optical depth $\tau=1$ as retrieved using the SPINOR 2D inversion code. Positive $v_{L O S}$ indicate flows moving away from the observer, and $\gamma_{L O S}=0^{\circ}$ indicate field lines pointing towards the observer. Adapted from Tiwari et al. (2015).

Ohmic diffusion, and turbulent diffusion (see e.g., Weiss 1966, Meyer et al. 1974, 1977, Petrovay and Moreno-Insertis 1997, Martínez Pillet 2002), the later including a diffusivity term with non-linear dependence on the magnetic field strength.

\subsection{General properties of sunspots}

Once a sunspot is fully developed, a characteristic photospheric morphology can be identified based on the brightness, comprising a dark central region (with brightness of $10-30 \%$ that of the quiet Sun, integrated over wavelength) known as the umbra, and a less dark region (brightness of $\sim 75-85 \%$ that of the quiet Sun) that surrounds either partially or completely the umbra, and which is called the penumbra.

The various brightness levels observed in a sunspot indicate differences in tempera- 
ture, and therefore different magnetic regimes (see e.g. Fig. 2.2); with the umbra being cooler than the penumbra and the latter being cooler on average than the quiet Sun. The relations between brightness/temperature and magnetic field strength as functions of the radial position (with respect to the sunspot's geometrical center) have been extensively studied in the photosphere (e.g., Lites et al. 1990, Keppens and Martínez Pillet 1996, Solanki 1993, Mathew et al. 2003, 2004, Tiwari et al. 2015).

Generally, the strongest magnetic fields in sunspots are concentrated within the umbra (an exception to this is presented in Chapter 3, cf. van Noort et al. 2013) and the maximum strength appears to depend on the sunspot size (e.g., Kopp and Rabin 1992, Collados et al. 1994, Solanki 1997, Schad and Penn 2010, Kiess et al. 2014). The magnetic field strengths typically vary between $2500-4000 \mathrm{G}$ in the umbra (Livingston 2002), with the largest umbral field ever recorded being nearly $6.1 \mathrm{kG}$ (Livingston and Harvey 2006). At the umbra-penumbra boundary the field strength is on average $1500-2500 \mathrm{G}$ while it is $500-1000 \mathrm{G}$ near the outer penumbral boundary (Lites et al. 1990, Skumanich et al. 1994, Westendorp Plaza et al. 2001b).

The magnetic field strength in a sunspot decreases with height in the atmosphere and, azimuthally averaged, it also decreases gradually with radial distance to the sunspot center (e.g. Joshi et al. 2017b, but see Joshi et al. (2017a) for counter-examples). Nonetheless, even if the field strength decreases rapidly outside the sunspot, the field continues to expand as a horizontal canopy towards higher layers in the atmosphere (e.g., Giovanelli and Jones 1982, Solanki et al. 1992b, 1994, Bruls et al. 1995). The inclination of the magnetic field also varies with radial distance, from almost vertical in the umbra to more inclined in the penumbra $\left(70-80^{\circ}\right.$ on average, with respect to the surface normal). Such differences in the mean inclination of the magnetic field change the way the convective motions interact with the magnetic field.

Sunspot magnetic fields are not force-free in the lower photosphere but nearly forcefree in higher atmospheric layers (e.g., Puschmann et al. 2010). The plasma $\beta$ (the ratio between the gas pressure and the magnetic pressure) is the key parameter to distinguish between force-free and non force-free magnetic field configurations. Generally, when $\beta \gg 1$, the plasma motions dominate the dynamics of the magnetic field resulting in non force-free (and therefore non-potential) configurations, while at $\beta \ll 1$, the magnetic field rules the plasma motions and it can eventually evolve into a potential configuration (e.g., Priest 1982). Borrero and Ichimoto (2011) found that the plasma $\beta$ values in sunspots comprise different regimes, having sufficiently small values in the high photosphere (between $\tau=10^{-2}$ and $10^{-3}$ ) to consider the magnetic field as nearly force-free there, while in the deep photosphere $(\tau=1), \beta \geq 1$ was found, meaning that the field is generally non force-free at those heights. Furthermore, different $\beta$ regimes were found at an intermediate height $\left(\tau=10^{-1}\right)$ such that the magnetic field is nearly force-free in the umbra but not in the penumbra. Nearly force-free fields can be approximated by potential fields in the absence of electric currents. Many theoretical models for sunspots have indeed considered potential fields by either placing the electric currents at the boundaries of sunspots (e.g., Simon and Weiss 1970, Meyer et al. 1977, Pizzo 1990) or by distributing them everywhere inside the sunspot (e.g., Pizzo 1986) so that the field is potential elsewhere. However, the large electric currents that have been detected within sunspots (e.g., Puschmann et al. 2010, Solanki et al. 2003, Socas-Navarro 2005) imply that their magnetic field is generally non-potential: $\nabla \times \vec{B} \neq 0$ (e.g., Borrero and Ichimoto 2011). 


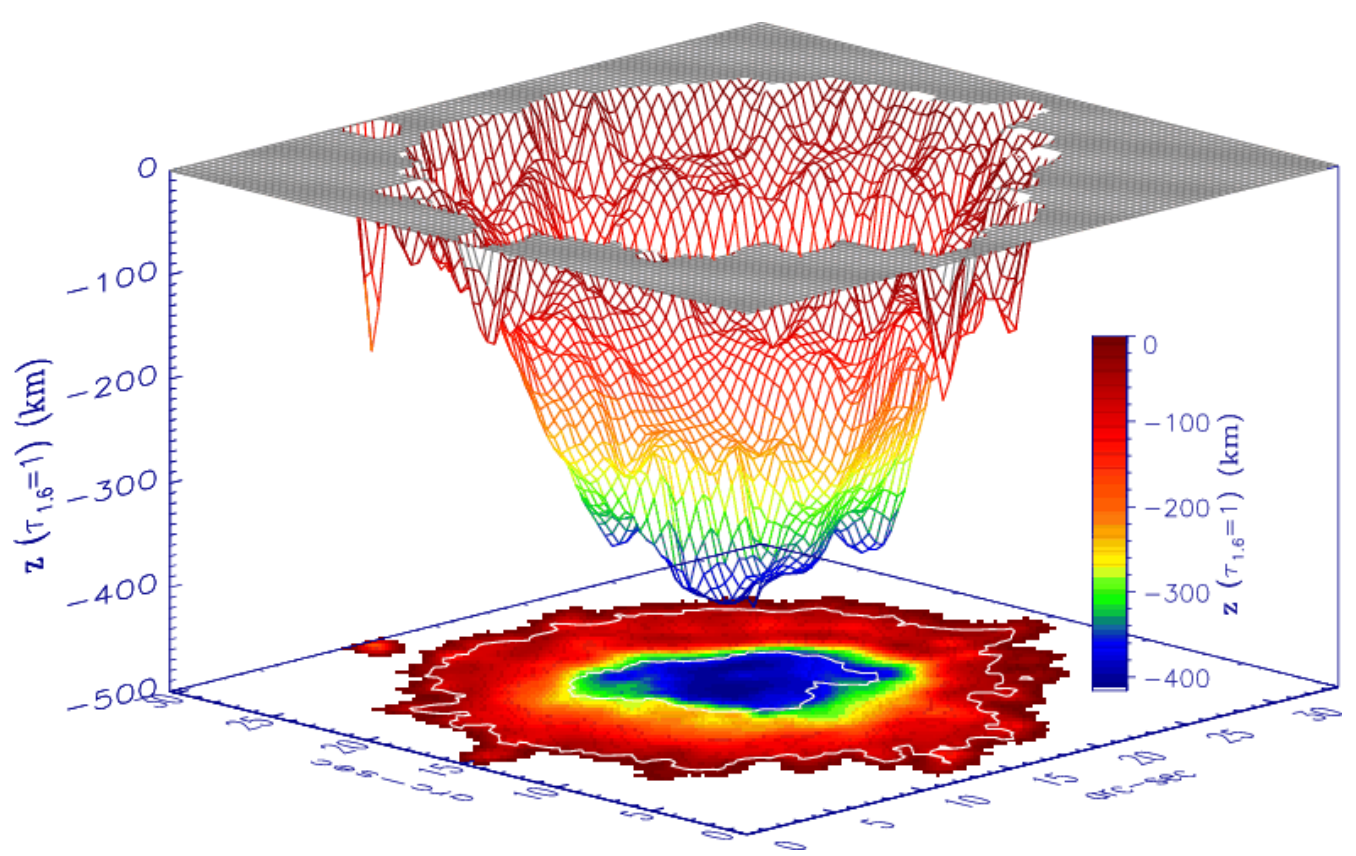

Figure 2.3: Computation of the Wilson depression $Z(\tau=1)$ at every point in an observed sunspot, neglecting curvature forces, by Mathew et al. (2004). They found an average depression of the $\tau=1$ surface of around $400 \mathrm{~km}$ in the umbra and around $120 \mathrm{~km}$ in the penumbra, with respect to the quiet Sun. The inner and outer contours represent the umbral and penumbral boundaries, respectively. From Mathew et al. (2004).

Sunspots host a wide variety of dynamic phenomena, such as large- and small-scale flows as well as different wave phenomena. In particular, in the penumbra the most distinctive gas flow is the Evershed flow, which is a horizontal flow pointing radially outwards in the photosphere, while higher up in the chromosphere an inward flow that has a significant downflowing component above the umbra is normally observed (see Section 2.6). Sunspot observations and modeling have also confirmed that large convection cells can form around sunspots, producing a radial outflow in an annular region of the surface around the sunspot, called moat flow, and inflows in the subsurface layers (e.g., Meyer et al. 1974, 1977). Furthermore, sunspots present different kinds of oscillations having different properties in the umbra and in the penumbra, as well as in the different layers of the atmosphere, which suggests that the wave properties depend on the magnetic configuration in good agreement with the predictions of MHD wave theory. While sunspots can present global oscillations with periods of the order of hours to days (e.g., Khomenko and Collados 2015), the most prominent oscillations in sunspots have periods of the order of a few minutes, e.g., typical $p$-mode periods of 5 minutes and 3 minutes chromospheric oscillations (Solanki 2003).

An important consequence of sunspots being large magnetic field concentrations confined by the external pressure exerted by the nearly field-free gas of the surrounding environment, is that, due to the radial balance between the gas and the magnetic pressures, the gas pressure as well as density and temperature in the umbra are lower than in the penumbra and quiet Sun. Given that both the net opacity and the $\mathrm{H}^{-}$bound-free opacity are very strongly sensitive to small temperature variations of the emitting medium, the 
latter results in the so-called Wilson depression, which is the deformation of the $\tau=1$ surface level within the sunspot regions.

Thus, taking the average quiet-sun temperature and the average geometrical height of its $\tau=1$ surface level as references, we can say that lower temperatures cause the opacity to decrease, and therefore one can see deeper into the Sun, while at higher temperatures we see higher layers in the atmosphere. In an average sense, sunspots produce the depression of the $\tau=1$ level whose depth increases towards the umbra (known as Wilson depression, see e.g., Bray 1964). The extent of the depression is not easy to determine and varies from sunspot to sunspot (e.g., Mathew et al. 2004). However, the true shape of such a surface would rather be extremely irregular when taking into account all the smaller scale structures in sunspots. An example of the complex morphology of the Wilson depression, as computed by Mathew et al. (2004) at every point in an observed sunspot, is shown in Figure 2.3.

\subsection{The umbra}

High resolution observations have revealed substantial fine-scale structure and dynamics in sunspots, both within umbral and penumbral regions (see e.g., a review by Thomas and Weiss (2004)). In the umbra (see e.g., Fig. 1.5), bright granular structures of small size are frequently observed (see e.g., Riethmüller 2013, for a discussion on different types, sizes and morphological properties) and are typically referred to as umbral dots.

Umbral dots are hotter than their umbral background (with temperature differences of $\sim 500-1500 \mathrm{~K}$ at $\tau=1$, e.g., Riethmüller et al. 2008, Socas-Navarro et al. 2004) and have slightly more inclined fields (Socas-Navarro et al. 2004) which are, however, still largely vertical as in the rest of the umbra. Signatures of convection have been found in umbral dots by, e.g., Riethmüller et al. (2008) and Riethmüller (2013), who reported the presence of upflows at the center of umbral dots (surrounded by downflow signatures) associated with a weakening of the magnetic field and an increase in temperature. Umbral dots are thought to be manifestations of localized magneto-convection taking place inside the umbra, as was shown to occur in 3D MHD simulations (Schüssler and Vögler 2006). In this scenario, the convective motions inside the umbra occur through vertical columns of reduced field strengths, where the gas reaching the surface cools down by radiative losses, pushing the magnetic field towards the sides before sinking back. At the top of the convective column, the increased density of the upflowing material could be responsible for the appearance of the central dark lane inside simulated umbral dots (Schüssler and Vögler 2006).

Another kind of umbral structures are the so-called light-bridges, which are seen as elongated bright structures dividing the umbra into two or more parts (see Fig. 1.5). Lightbridges are often observed after the merging of two or more sunspots or pores during the growth phase of the final sunspot. However, other types of light-bridges are seen to form from extensions of the penumbra into the umbra, which grow until they cross the whole umbra. Some light bridges comprise granular-like substructures that are thought to be remnants of the quiet Sun granulation (e.g., Hirzberger et al. 2002, Lagg et al. 2014). Similar to umbral dots, the magnetic field is observed to be weaker and slightly more inclined in light bridges as compared to the surrounding umbra (Beckers and Schröter 
1969, Rüedi et al. 1995, Jǔrcák et al. 2006) and the origin of the central dark lane in light bridges are thought to be the same as in umbral dots (e.g., Borrero and Ichimoto 2011).

To understand the presence of convection in sunspot umbrae and its subsurface layers, the two different proposed theoretical models for sunspot subsurface structure need to be explored: the monolith model (Cowling 1957, Gokhale and Zwaan 1972, Meyer et al. 1974, 1977), which describes a sunspot as a single flux tube, and the cluster model (Parker 1979), in which the sunspot magnetic field would split into many small flux tubes (so-called spaghetti-shape) below the surface due to the interchange/fluting instability (Parker 1975). In both cases, the surface signatures of convection would be similar to the ones observed in umbral dots and light bridges. However, while the monolith model implies that umbral convection can only occur as a surface effect surrounded by the strong umbral field, in the cluster model, the field-free regions between the small flux tubes would allow the umbral convection to reach deep regions in the convection zone. None of these models can be tested with sprectropolarimetric observations. Distinguishing between them requires the further development of local helioseismology techniques as well as numerical simulations to better understand the subsurface structure of sunspots (e.g., Solanki 2003, Gizon and Birch 2005, Schüssler and Vögler 2006, Moradi et al. 2010, Borrero and Ichimoto 2011).

\subsection{On the existence of a penumbra}

The development of a penumbra, a filamented region with an on average inclined magnetic field that surrounds either partially or completely the dark umbra, is what differentiates sunspots from pores. However, the physical mechanism responsible for penumbra formation, as well as its relation with the start of the Evershed flow, remains unclear given that the observational study of this process demands the highest temporal, spatial and spectral resolutions since its early stages of formation (Thomas and Weiss 2004) and its simulation proves to be very challenging (e.g., Cheung et al. 2010).

There have been only a few reported observations of penumbra formation (e.g., Leka and Skumanich 1998, Yang et al. 2003, Schlichenmaier et al. 2010a,b, 2012, Romano et al. 2014, Murabito et al. 2016). Overall, the formation process of penumbrae is very fast, taking from minutes to a few hours, and it occurs in parts. It has been found by Schlichenmaier et al. (2010a), Schlichenmaier et al. (2010b) and Rezaei et al. (2012) that initially the penumbra cannot form on the side pointing towards the opposite polarity flux of the active region due to ongoing flux emergence. Generally, the penumbra starts forming on the side of the proto-spot located farthest to the opposite polarity flux of the active region, and the newly formed penumbral sectors host an Evershed flow soon after its formation. Nevertheless, during the early stages of penumbra formation, flow regions with opposite signs to the Evershed flow have been observed, i.e. inflows towards the proto-spot which are thought to play some role during the formation of the penumbra (e.g., Schlichenmaier et al. 2012, Romano et al. 2014).

According to Zwaan (1992) the formation of the penumbra is not linked to a dramatic increase of the magnetic flux in the sunspot, while the findings of Leka and Skumanich (1998), Schlichenmaier et al. (2010a), Schlichenmaier et al. (2010b) and Rezaei et al. (2012) all agree that the formation of a penumbra does not occur at the expense of the 
umbral magnetic flux. While it has been suggested that the penumbra is formed due to the emergence of horizontal fields which get trapped in the photosphere due to the sunspot magnetic canopy lying above (e.g., Leka and Skumanich 1998), there have been some observations that show that the signatures of a forming penumbra appear first in the chromosphere and later in the photosphere (e.g., Shimizu et al. 2012, Romano et al. 2013, 2014) suggesting that an increase on the inclination and the sinking of some canopy field lines could trigger the penumbra formation (e.g., Murabito et al. 2016).

Theoretical studies by Rucklidge et al. (1995) have predicted that values between $1-7 \times 10^{20} \mathrm{Mx}$ of the total magnetic flux are necessary for the development of a penumbra around a pore. These values are supported by some observational studies, e.g., Leka and Skumanich (1998) found a threshold of $1-1.5 \times 10^{20} \mathrm{Mx}$ above which penumbrae can form, while Zwaan (1987) and Rezaei et al. (2012) found threshold values of $5 \times 10^{20} \mathrm{Mx}$ and $4 \times 10^{20} \mathrm{Mx}$, respectively. However, the findings of Kuckein et al. (2012), Jǔrcák et al. (2014) and Zuccarello et al. (2014) suggest that the total magnetic flux does not provide a condition for the penumbra formation, but the configuration of the magnetic field does. Rucklidge et al. (1995) also showed that the magnetic field inclination should exceed the critical value $\gamma_{c r i t}=45^{\circ}$ so that the penumbra is formed, a value that was supported by the recent MHD simulations of Rempel et al. (2009a).

Also, Rempel (2012a) found that the formation of the penumbra is favored when the horizontal component of the magnetic field is increased at the upper boundary of the simulation domain. These results support the scenario in which horizontal magnetic fields in the chromosphere trigger the formation of the penumbra. However, Chen et al. (2017) recently presented a sunspot simulation based on the emergence of flux bundles generated in a solar convective dynamo, in which filamentary penumbral structures form during flux emergence without having to impose a strong field inclination at the top of the simulation box. Nonetheless, these simulations produce a persistent mass inflow along the penumbral filaments (opposite in direction to the typically observed Evershed flow) which is possibly related to the emerging magnetic flux.

Based on the study of stable sunspots with fully-developed penumbrae, Jǔrcák (2011) found that the umbra-penumbra boundary can be characterized by a critical value of the magnetic field, $B_{v e r}^{\text {crit }} \approx 1.8 \mathrm{kG}$, and suggested that this can be a crucial parameter to understand the different mechanisms occurring in umbrae and penumbrae. According to theoretical calculations by Chandrasekhar (1961), convection is only affected by the vertical component of the magnetic field, while the horizontal field component only modifies the geometry of the convective cells (see also Thomas and Weiss 2008). Thus, Jǔrcák (2011) proposed that the penumbral form of magneto-convection (referring to the Evershed flow) takes place when $B_{v e r}<B_{v e r}^{\text {crit }}$.

The necessary conditions for penumbrae formation can then be summarized as: a critical value for the total magnetic flux inside the proto-spot, for the field strength and for the field inclination, as well as a relatively 'quiet' surrounding magnetic environment (e.g., Rezaei et al. 2012). However, it is not known if these conditions are sufficient. 


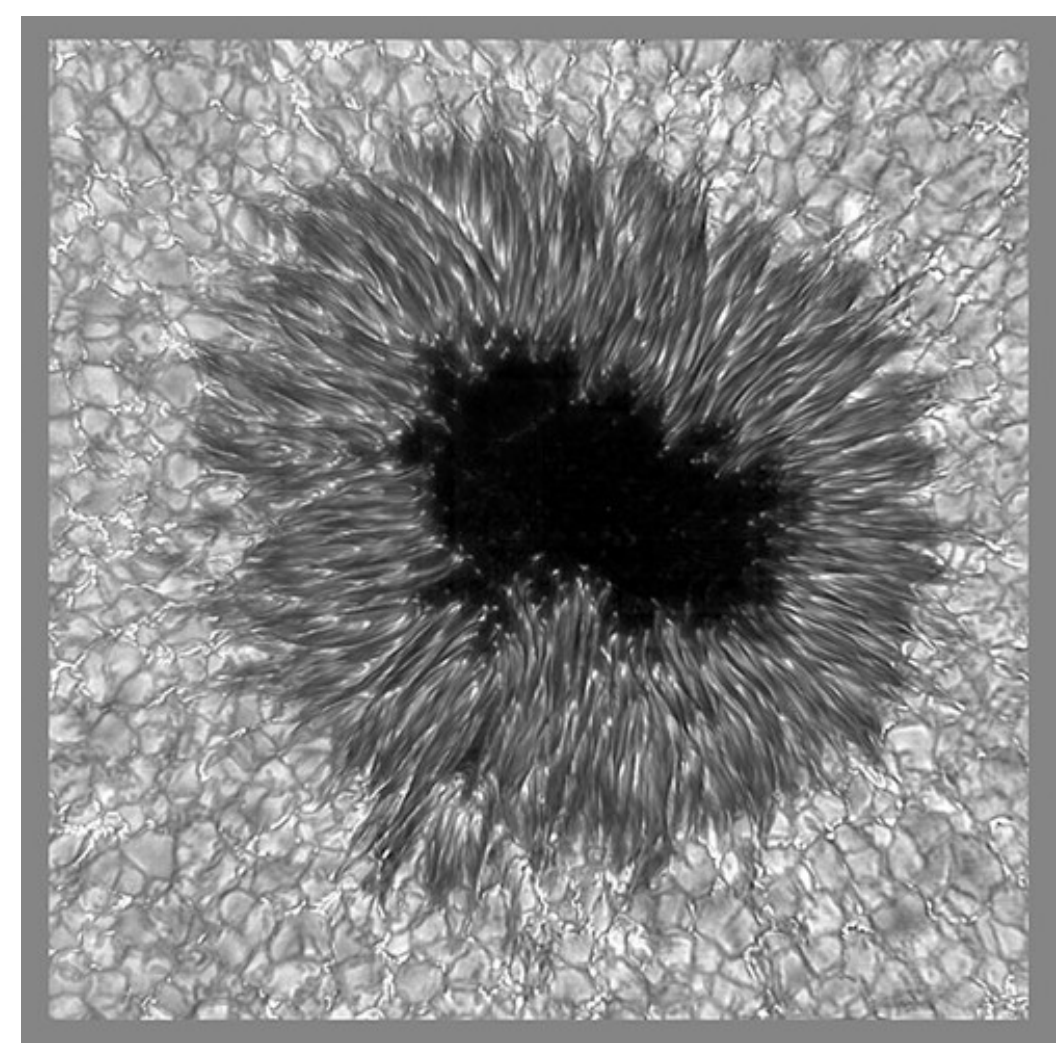

Figure 2.4: Continuum image of a sunspot taken at the Dunn Solar Tower at the National Solar Observatory at Sacramento Peak, New Mexico. Source: F. Woeger (KIS), C. Berst, M. Komsa (NSO/AURA/NSF).

\subsection{Penumbral fine structure}

Sunspot penumbrae display a filamentary pattern in all their physical quantities, such as in intensity and temperature, flow velocity, and magnetic field (see e.g. Fig. 2.2), which requires high spatial resolution spectropolarimetric measurements, much below $0.5^{\prime \prime}$, to be adequately resolved.

The penumbral filamented intensity pattern, as seen in the continuum images (e.g. Fig. 2.4), consists of radially aligned elongated bright and dark filaments, which are arranged in a seemingly alternating way in the azimuthal direction, and whose averaged intensity lies between 75 and $85 \%$ of the intensity of the quiet Sun.

The bright filaments are more easily identifiable near the inner penumbral boundary, where their contrast with the dark umbra is larger. They start in bright teardrop-shaped regions known as penumbral grains, which correspond to the heads of the filaments and which display a radial, predominantly inward motion (e.g., Muller 1976, Sobotka et al. 1995, Denker 1998). The penumbral bright filaments extend radially towards the sunspot periphery with a steady decrease in intensity, but their end parts, known as filaments' tails, can display an enhancement in intensity (e.g., Bharti et al. 2010, Tiwari et al. 2013). Some of the filaments extend over the entire penumbra, while others seem to vanish within it. Furthermore, some filaments originate farther from the umbra-penumbra boundary (Fig. 2.5), so that the filaments can be classified into inner filaments if they originate close to 


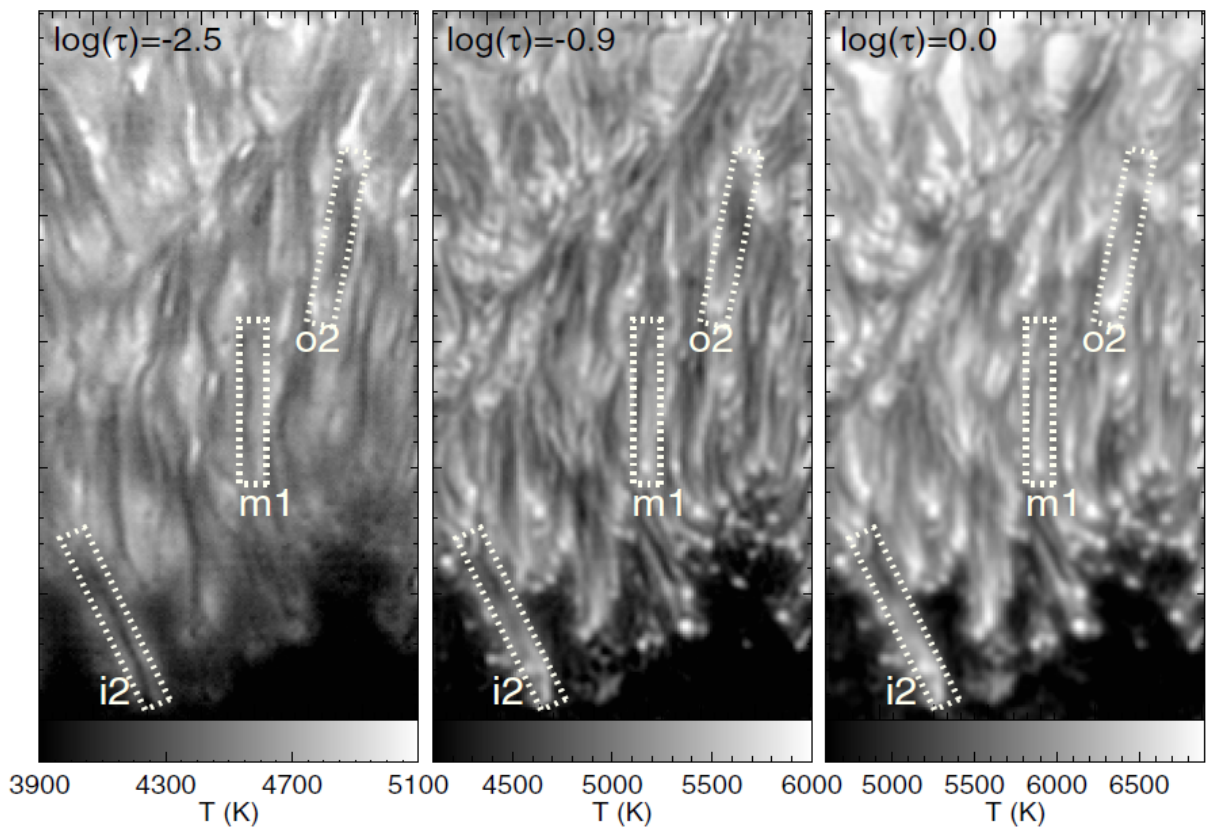

Figure 2.5: Classification of penumbral filaments according to their location within the penumbra. The dotted boxes marked as $i 2$ highlight an inner filament, while the boxes marked as $m 1$ and $o 2$ enclose a middle and outer filament, respectively. The maps show the temperature in a part of the sunspot penumbra of AR NOAA 10933 as retrieved with SPINOR 2D inversions at $\log \tau=-2.5$ (left panel), -0.9 (middle panel) and 0 (right panel). From Tiwari et al. (2013).

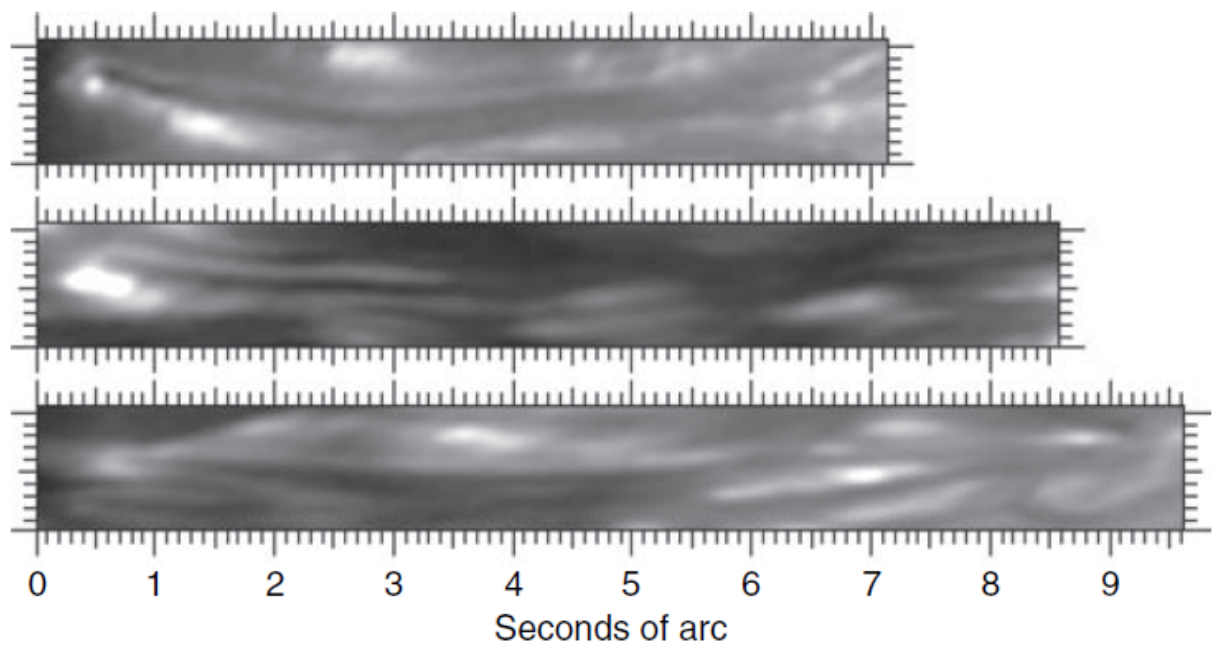

Figure 2.6: Example of three bright penumbral filaments of different sizes and with dark central lanes observed with the Swedish Solar Telescope (SST) at a spatial resolution of $\sim 0^{\prime \prime} .1$ near the umbra. From Rouppe van der Voort et al. (2004). 


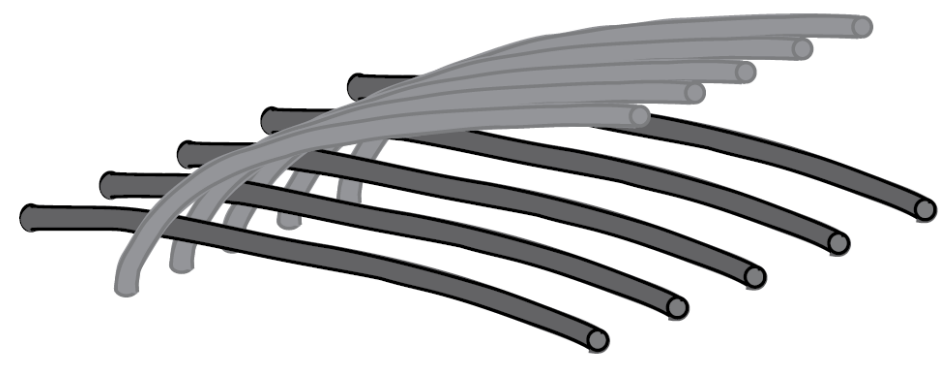

Figure 2.7: Sketch of the interlocking-comb configuration of sunspot penumbrae. From Weiss et al. (2004).

the umbra-penumbra boundary, middle filaments if they originate in the inner-to-middle parts of the penumbra, and outer filaments if they originate in the middle-to-outer parts of the penumbra (Tiwari et al. 2013). Typical widths of the bright penumbral filaments have been found to be of the order of $0^{\prime \prime} .3-0^{\prime \prime} .6$ with lifetimes from a few minutes to $\sim 4$ hours (Solanki 2003, and references therein). Nonetheless, Rouppe van der Voort et al. (2004) did not find any preferred width for the bright penumbral filaments and argued that unresolved filaments with widths below $0^{\prime \prime} .1$ can also exist.

Scharmer et al. (2002) realized that some bright penumbral filaments display a dark core, such as in the examples shown in Figure 2.6. Such dark cores are typically best observed in inner filaments (e.g., Bellot Rubio et al. 2005, 2007, Langhans et al. 2007, Rimmele 2008) and in the middle and upper photospheric layers (see, e.g., the inner filament $i 2$ in Fig. 2.5, from Tiwari et al. 2013, whose dark core is better observed above $\log \tau=-0.9$ ). Spruit and Scharmer (2006) suggested that the dark cores in penumbral filaments could be a consequence of field-free gaps above which the iso- $\tau$ surface is elevated to higher and cooler layers due to a higher gas pressure that results from the absence of magnetic pressure. Similar explanations by Borrero (2007) and Ruiz Cobo and Bellot Rubio (2008) describe the dark cores as a consequence of an elevated iso- $\tau$ surface, but produced as a consequence of an increased density due to the weaker fields in horizontal flux tubes.

The determination of the magnetic field configuration in the penumbra requires the analysis of the full Stokes vector at high spatial resolution and is necessary to understand the underlying physics related to the filamented intensity patterns of penumbrae. An azimuthal variation of the magnetic field strength was already noticed by Beckers and Schröter (1969), who found that the magnetic field in darker filaments is stronger than it is in the brighter filaments. Schmidt et al. (1992) and Title et al. (1993) noticed that also the field inclination varies azimuthally. These findings where later confirmed first by Lites et al. (1993) who performed depth independent inversions (Milne-Eddington) of the full Stokes vector and found azimuthal variations of $\Delta \gamma=10^{\circ}$ and $\Delta B=100 \mathrm{G}$ on spatial scales of the order of $1^{\prime \prime}$, the stronger magnetic fields being more vertical (so-called spines) and the weaker fields more inclined (so-called intra-spines). This configuration was referred to as fluted penumbra (e.g., Skumanich et al. 1994, Solanki 2003). However, there has been a lot of controversy regarding the relation of spines and intra-spines with the dark penumbral lanes and the bright filaments. Such controversies were mainly driven 

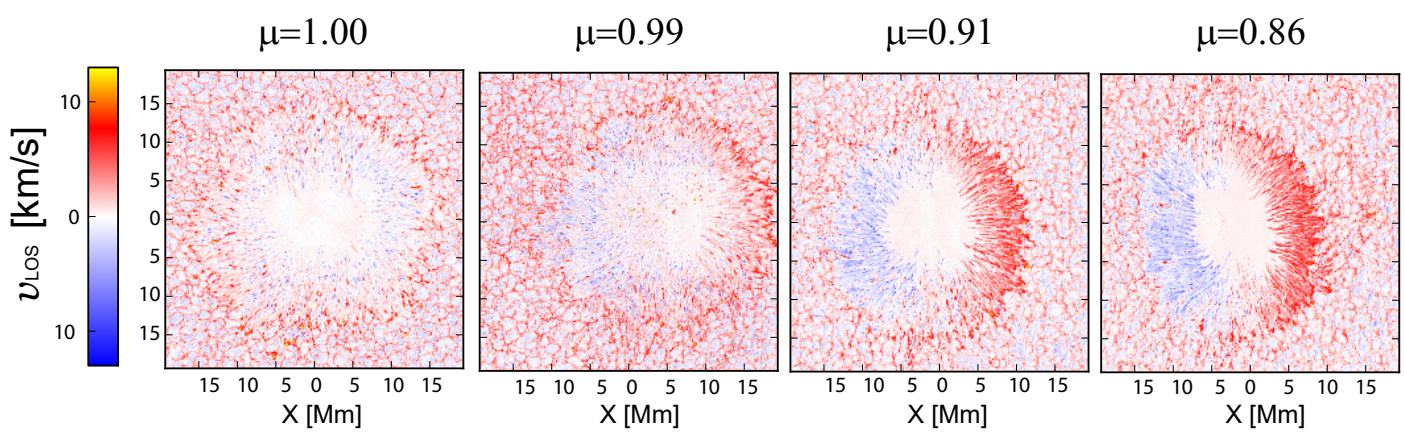

Figure 2.8: The Evershed effect observed in AR NOAA 10933 as it moves away from the solar disk center. The maps show the LOS velocity of the sunspot at 4 different positions on the solar disk (from $\mu=1$ to $\mu=0.86$ ) as retrieved by SPINOR 2D inversions, at $\tau=1$, of Hinode SOT/SP spectropolarimetric observations. Positive $v_{L O S}$ indicate flows moving away from the observer. The blue-shifted (negative $v_{L O S}$ ) part of the penumbra is facing the solar disk center. The umbra was set at rest. Courtesy of Michiel van Noort.

by low spatial resolution observations, a lack of information on the full Stokes vector, as well as the use of simple estimation methods to infer the magnetic field strength and inclination.

It was later shown that the spines are found in the darker penumbral lanes, while the intra-spines tend to be bright filaments (e.g., Borrero and Ichimoto 2011, Tiwari et al. 2013). The spines and intra-spines form the so-called interlocking comb structure of the penumbra (Thomas and Weiss 1992, Solanki and Montavon 1993), which refers to the different inclinations interlaced with each other in the azimuthal direction (Fig. 2.7). However, Tiwari et al. (2013) showed that the brightness and the magnetic field inclination also display significant changes from the head to the tail of a filament (intra-spine). According to their findings, the filament's head is a brighter region where the field is almost vertical and has similar inclinations as in the dark spines. The field rapidly bends over so that its inclination increases with radial distance, and lies almost horizontally along the body of the filament whereas the intensity steadily decreases. Moreover, the filament's tail can show an enhancement in intensity and the magnetic field becomes stronger, more vertical, and it submerges back into the solar interior with an opposite polarity to that of the head. In contrast, the positive field divergence of the magnetic field in the dark spines suggests that they possibly connect with regions far from the sunspot to form coronal loops over the active region (Borrero and Ichimoto 2011).

\subsection{The Evershed flow}

Another interesting, even fundamental aspect of penumbrae concerns their flow velocity field which, similarly to the penumbral brightness and magnetic field, also exhibits a filamentary structure.

The Evershed flow is the most outstanding dynamic phenomenon in sunspot penum- 
brae and was first noticed by John Evershed in 1909 as a systematic wavelength shift of various photospheric spectral lines in penumbrae (Evershed 1909). He found that, when the sunspots were located far from the disk center, the spectral lines were blue-shifted over the part of the penumbra facing the disk-center, while they were red-shifted over the limb-side penumbra. Moreover, when the sunspot was located near the disk center, the strong wavelength shifts vanished. This effect (since its discovery named the Evershed effect; an example is shown in Fig. 2.8) was interpreted as the result of an almost horizontal and radial outflow of material occurring in sunspot penumbrae, the Evershed flow.

These fast outflows (typical spatially averaged speeds being $1-2 \mathrm{~km} \mathrm{~s}^{-1}$ ) are usually subsonic, although supersonic flows have also been observed (e.g. Wiehr 1995, del Toro Iniesta et al. 2001, Bellot Rubio et al. 2004, Borrero et al. 2005), and are found to be concentrated in regions of weaker magnetic field (Degenhardt and Wiehr 1991, Schmidt et al. 1992, Title et al. 1993, Rimmele 1995, Westendorp Plaza et al. 2001a, Borrero et al. 2005, Ichimoto et al. 2007). Inversions of spectropolarimetric observations indicate that the Evershed flow is parallel to the magnetic field (e.g., Bellot Rubio et al. 2003, 2004), as expected for a highly conducting plasma, and that it is carried along the nearly horizontal fields in the bright penumbral filaments (e.g., Borrero et al. 2004, 2005, Bello González et al. 2005). Furthermore, the Evershed flow is height dependent: in the photosphere, the line shifts decrease rapidly with height of line formation (e.g., St. John 1913, Maltby 1964, Börner and Kneer 1992) and, in the chromosphere, the line shifts change sign, with a blue-shifted limb-side penumbra and a red-shifted center-side penumbra (St. John 1913, Börner and Kneer 1992, Tsiropoula 2000). This is taken to be the signature of an inflow of material (so-called chromospheric inverse Evershed flow). Most of the mass flowing outwards in the photosphere returns to the solar interior within the penumbra, in opposite polarity downflow channels (Westendorp Plaza et al. 1997, 2001a), although a part of the Evershed flow is believed to continue in the canopy of the sunspot (Solanki et al. 1994).

The Evershed flow does not only produce the wavelength shift of the photospheric spectral lines, but it also causes lines asymmetries, which were already noticed by, e.g., St. John (1913), Holmes (1963) and Schröter (1965). Such Doppler shifts and asymmetries are observed in all 4 Stokes profiles (e.g., Shurcliff 1962, Beckers and Schröter 1969) and are a consequence of the horizontal gradients caused by the filamentary structure of sunspot penumbrae (given that different structures can be unresolved) as well as of gradients along the LOS in the flow velocity, magnetic field strength, and field inclination (e.g., Sánchez Almeida and Lites 1992). In particular, the observed net circular polarization $\left(\mathrm{NCP}=\int V(\lambda) d \lambda\right.$, which is a measure of the asymmetry of the Stokes $V$ profiles) of photospheric spectral lines in the penumbra gave rise to different models aimed to explain the penumbral configuration of the magnetic field and the flow structure of penumbrae.

\subsubsection{Some penumbral models}

The embedded flux-tube model, is empirical in nature and was proposed by Solanki (1993). It consists of a penumbra formed by horizontal magnetic flux tubes representing the intraspines, which are embedded in more vertical background magnetic fields (spines) and carry most of the Evershed flow (left panel of Fig. 2.9). The downward pumping mechanism associated with the granular convection outside the sunspots (Thomas et al. 2002) was considered by Solanki (1993) to explain the origin of field lines that return into the 

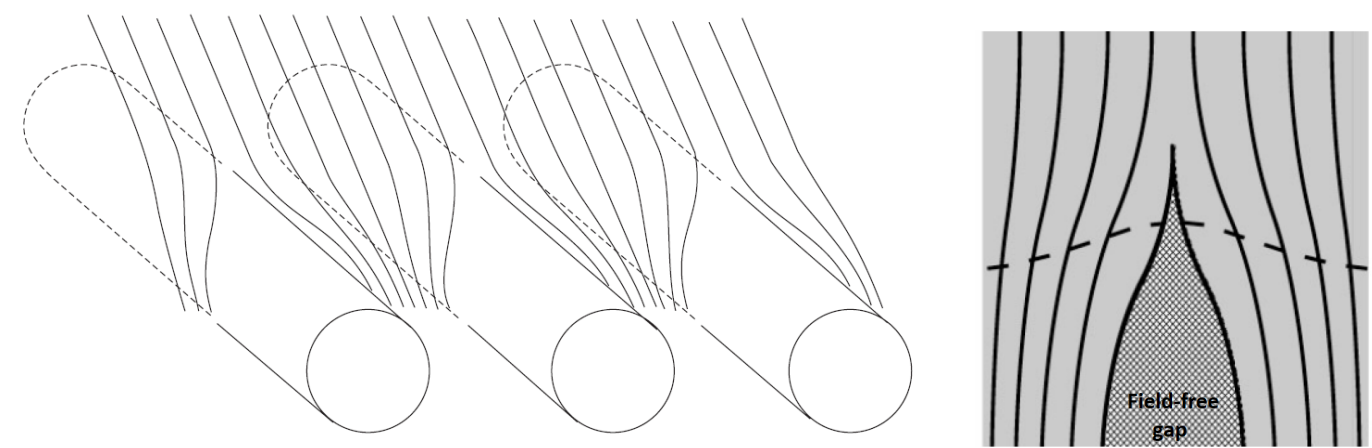

Figure 2.9: Two different penumbral models. (Left:) The embedded flux-tube model proposed by Solanki (1993). The horizontal cylinders in the sketch represent the fluxtubes forming the intra-spines, in which the Evershed flow is confined, and the more vertical solid lines stand for the magnetic field configuration in the spines. From Solanki (2003). (Right:) The field-free gap model proposed by Spruit and Scharmer (2006). The sketch represents a vertical cross section of a field-free gap (filament) with a vanishing vertical field component on its top, within which a protrusion of convection leads to the Evershed flow, and which are surrounded by vertical flux bundles. Adapted from Spruit and Scharmer (2006).

solar surface at the outer penumbra, by acting on magnetic fields that form the low-lying horizontal flux tubes. In this scenario, the Evershed flow is the result of siphon flows occurring along each arched magnetic flux tube. This model also uses a three-layered structure of the magnetic field, with a horizontal flux sheet as the central layer, to explain the observed asymmetric Stokes $V$ profiles in penumbrae and the center-to-limb variation of the NCP (e.g., Solanki and Montavon 1993).

The moving flux tube model or hot rising flux tube model was proposed by Schlichenmaier et al. (1998a) and Schlichenmaier et al. (1998b) to explain the increased temperature of the penumbra relative to the umbra, and its relation with the Evershed flow. This model considers a 1D magnetic flux tube that evolves in a 2D background by assuming that all the physical variables are constant across the tube and they can only vary along the tube. Overall, the model proposes that the penumbra is heated by a hot plasma that rises due to buoyancy from below towards the surface and flows through thin magnetic flux tubes, where the gas cools down by radiative losses when it reaches the $\tau=1$ surface and produces a gas pressure gradient along the flux tube, thereby driving the Evershed flow. Furthermore, the model describes the Evershed flow as a field-aligned flow in the interior of the flux tubes. This model was later extended into a 3D model by Schlichenmaier and Schmidt (2000) to show that the azimuthal variations of the magnetic field vector can reproduce the observed asymmetries of the NCP with respect to the line-of-symmetry of the sunspot.

Another model proposed to explain the configuration of the magnetic field in the penumbra is the field-free gap model (Choudhuri 1986, Scharmer and Spruit 2006, Spruit and Scharmer 2006). In this model, the bright penumbral filaments are described as radially elongated field-free regions, separated by a potential magnetic field, where the nonmagnetized hot gas that rises to the surface due to convection can protrude into a background with more vertical fields (right panel of Fig. 2.9). This causes the field-free gaps 


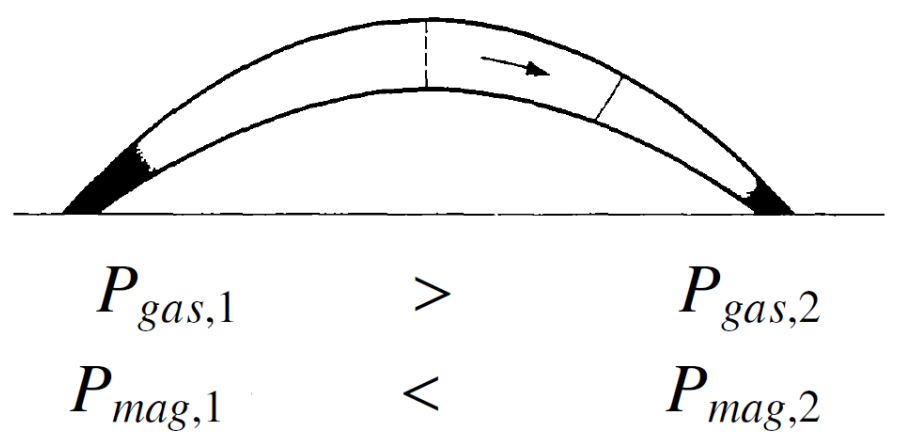

Figure 2.10: Sketch of a siphon flow in a magnetic flux tube. A difference between the magnetic field strength at the footpoints of an arched flux tube results in a gas pressure gradient which drives the flow along the flux tube (the arrow indicates the flow direction). Adapted from Meyer and Schmidt (1968a).

to appear as elevated structures in the penumbra. The Evershed flow is then interpreted as the horizontal component of overturning convection in the radial direction. Furthermore, as a consequence of continuity conditions, the vertical component of the magnetic field must vanish at the boundary between the field-free gaps and the vertical field background, resulting in a totally horizontal field at the top of the penumbral filaments (intra-spines).

Although all these models succeed in their own way in describing many observational aspects of penumbrae, they all suffer shortcomings. One important aspect that all the proposed penumbral models have to deal with, is the problem of penumbral heating, i.e., they must consider a mechanism of energy transport that can maintain the penumbral brightness in the surface between $75-85 \%$ of the average brightness in the quiet Sun.

Both the embedded flux-tube model and the hot rising flux tube model can explain the observed asymmetries of the Stokes profiles. In particular, the hot rising flux tube model can explain the inward migration of penumbral grains and predicts convective flows occurring along the penumbral filaments (radial convection). However, such radial convection can supply the necessary heat to the penumbra only if the gas travels small distances before turning into downflows. This is a consequence of the rapid radiative cooling processes that are considered in the scope of this model (Schlichenmaier et al. 1999). In contrast, albeit the field-free gap model does not predict a preferential radial direction for the convective upflows and downflows in the penumbra, it does provide an efficient heat transport given that the convective motions occur along the entire filament. The upflows are concentrated at the filament's central axis and the downflows occur azimuthally, i.e., towards the filament's edges (azimuthal or overturning convection). However, the fieldfree gap model does not provide a physical explanation for the observed preferential radial direction of the Evershed flow and neither for the nature of the filament's endpoints. Moreover, the large Doppler shifts observed in the polarization signals of photospheric spectral lines in the penumbra suggest that the Evershed flow is a magnetized gas flow (Borrero and Solanki 2008), and therefore these observations are not compatible with the field-free gap description of the penumbral filaments. 


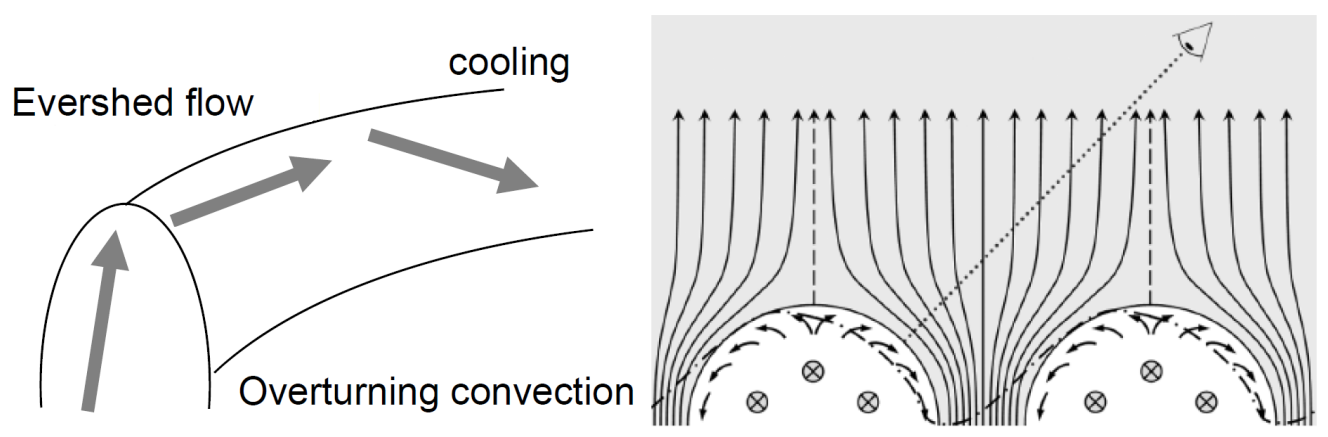

Figure 2.11: Left: Sketch illustrating the radial convection in a penumbral filament. The gray arrows indicate the direction of the convective gas, which flows upwards due to convection in the filament's head; it is then deflected radially outwards by the horizontal field along the filament's body; in the way, the gas cools down by radiative losses and it finally sinks back into the solar surface. Adapted from Meyer and Schmidt (1968a). Right: The sketch shows a vertical cross section of two filaments (semi-circles) where the magnetic field is horizontal (crosses indicate a field going into the plane of the paper). The arrows inside the filaments indicate the direction of the lateral convective flow. The arrows embedded into the gray areas represent the field lines of the surrounding spines. From Zakharov et al. (2008).

\subsubsection{Driving forces of the Evershed flow}

The origin and the physical mechanisms driving the Evershed flow have also been the subject of intense debate. All the theoretical models proposed to explain the complex structure of the penumbra include also an intimately connected description of the Evershed flow. Some models describe it as a siphon flow driven by a gas pressure difference between the footpoints of arched magnetic flux tubes (e.g., Meyer and Schmidt 1968a, Degenhardt 1989, Thomas and Weiss 1992, Montesinos and Thomas 1997, Thomas et al. 2006). These models have also considered the geometrical effects of the magnetic flux tubes and the radiative cooling processes. In such a picture, the footpoints of the magnetic flux tube must be in total pressure balance with each other at a given geometrical height, i.e.:

$$
P_{g a s, 1}+\frac{B_{1}^{2}}{8 \pi}=P_{g a s, 2}+\frac{B_{2}^{2}}{8 \pi}
$$

An outward flow of gas along a penumbral filament requires the gas pressure in the inner footpoint (labeled as 1 in the sketch shown in Fig. 2.10) to be larger than at the outer footpoint (labeled as 2), i.e. $P_{g a s, 1}>P_{g a s, 2}$, and therefore the magnetic field to be stronger in the outer footpoint than in the inner one, i.e. $B_{2}>B_{1}$.

Given that the magnetic field strength, on average, decreases radially outwards from the inner edge of the penumbra, the condition of $B_{2}>B_{1}$ in a penumbral filament forced the flux tube models to locate the outer footpoint outside the sunspot in regions of enhanced magnetic fields. Thus, by placing the outer footpoint in a magnetic element where $B$ can be of the order of $1.5 \mathrm{kG}$, so that the inner footpoint (located within the penumbra) harbors weaker fields than the outer footpoint, and therefore outwardly directed siphon flows can occur as is observed in the penumbra. However, the field strength 

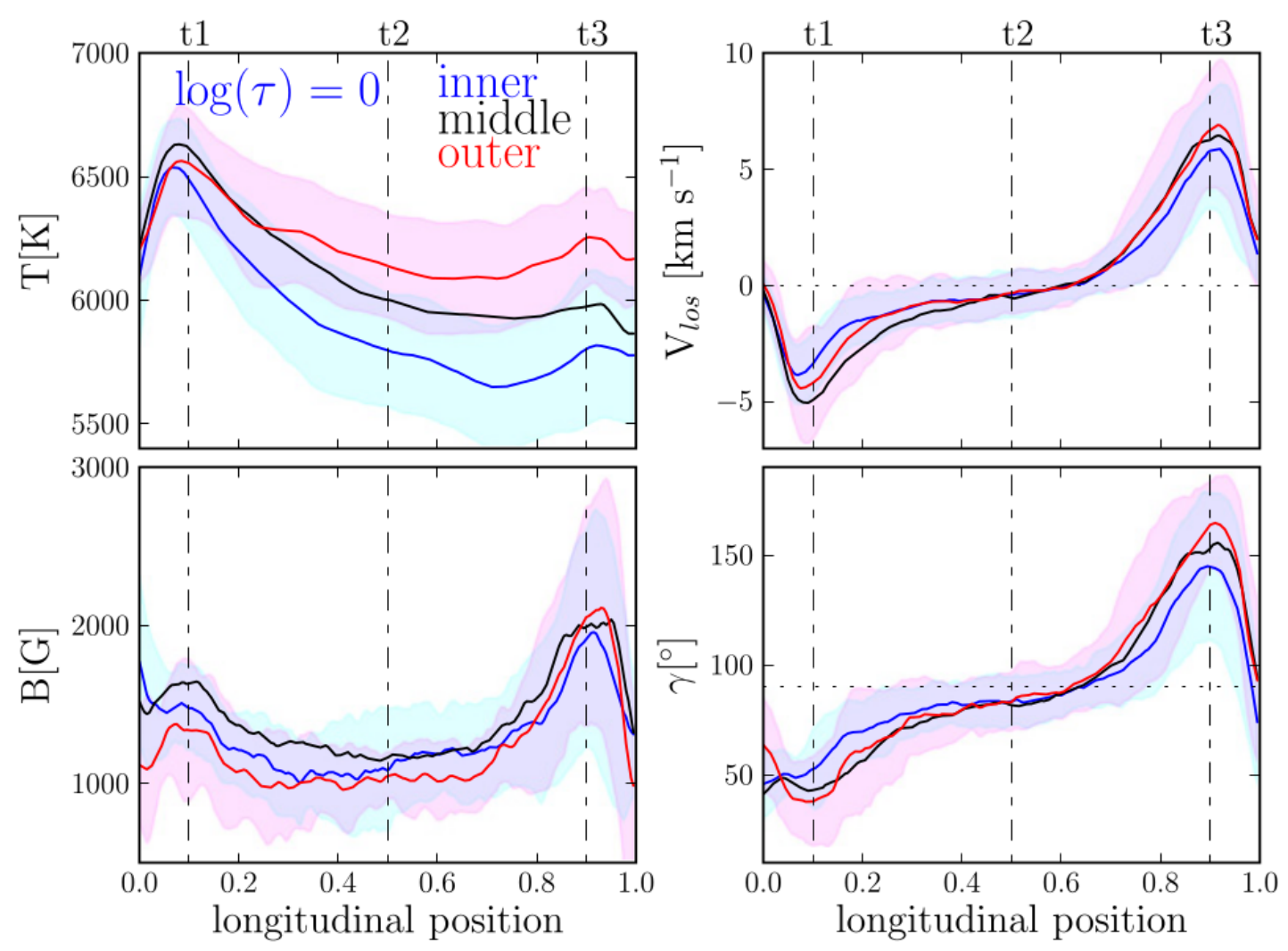

Figure 2.12: Average variation of temperature $T$, LOS velocity $v_{L O S}$, magnetic field strength $B$ and inclination $\gamma$ at $\tau=1$ along the central axes of three 'standard filaments' which were created by averaging a set of 20 inner filaments (blue), 20 middle filaments (black) and 20 outer filaments (red) in a sunspot located near the disk center. The vertical cuts labeled as $t 1$ and $t 3$ correspond to the head and the tail of the filaments respectively. The standard deviation of each filament set are indicated by the shaded areas of the respective color, except for the middle filaments. The $x$-axis shows the normalized length scale of the filaments. From Tiwari et al. (2013).

in the penumbra can be quite high, exceeding $2 \mathrm{kG}$ near the inner edge, where outward directed Evershed flows are seen. This makes such a scenario implausible.

Recently, Tiwari et al. (2013) performed a detailed analysis of the thermal, flow velocity and magnetic field structures of individual filaments located at different radial distances within the penumbra (i.e., they considered inner, middle and outer filaments as shown in Fig. 2.5) by using SPINOR 2D inversions of spectropolarimetric data from Hinode SOT/SP. They found a striking uniformity of the penumbral properties (Fig. 2.12) and provided, for the first time, a unified observational picture of the penumbral filaments found anywhere in the penumbra. They observed hot upflows in the filaments' head at optical depth unity $\left(T \approx 6600 \mathrm{~K}\right.$ and $v_{L O S}=5 \mathrm{~km} \mathrm{~s}^{-1}$ on average and up to $11 \mathrm{~km} \mathrm{~s}^{-1}$ in individual filaments) which gradually become narrower with distance along the filament while the temperature decreases. At the filament's tail, they observed concentrated supersonic downflows $\left(v_{L O S}=7 \mathrm{~km} \mathrm{~s}^{-1}\right.$ on average and up to $19 \mathrm{~km} \mathrm{~s}^{-1}$ in individual filaments) and an enhancement in temperature. The gas flowing in the tails is nonetheless cooler than the gas in the heads. The authors suggest that the increase in temperature 


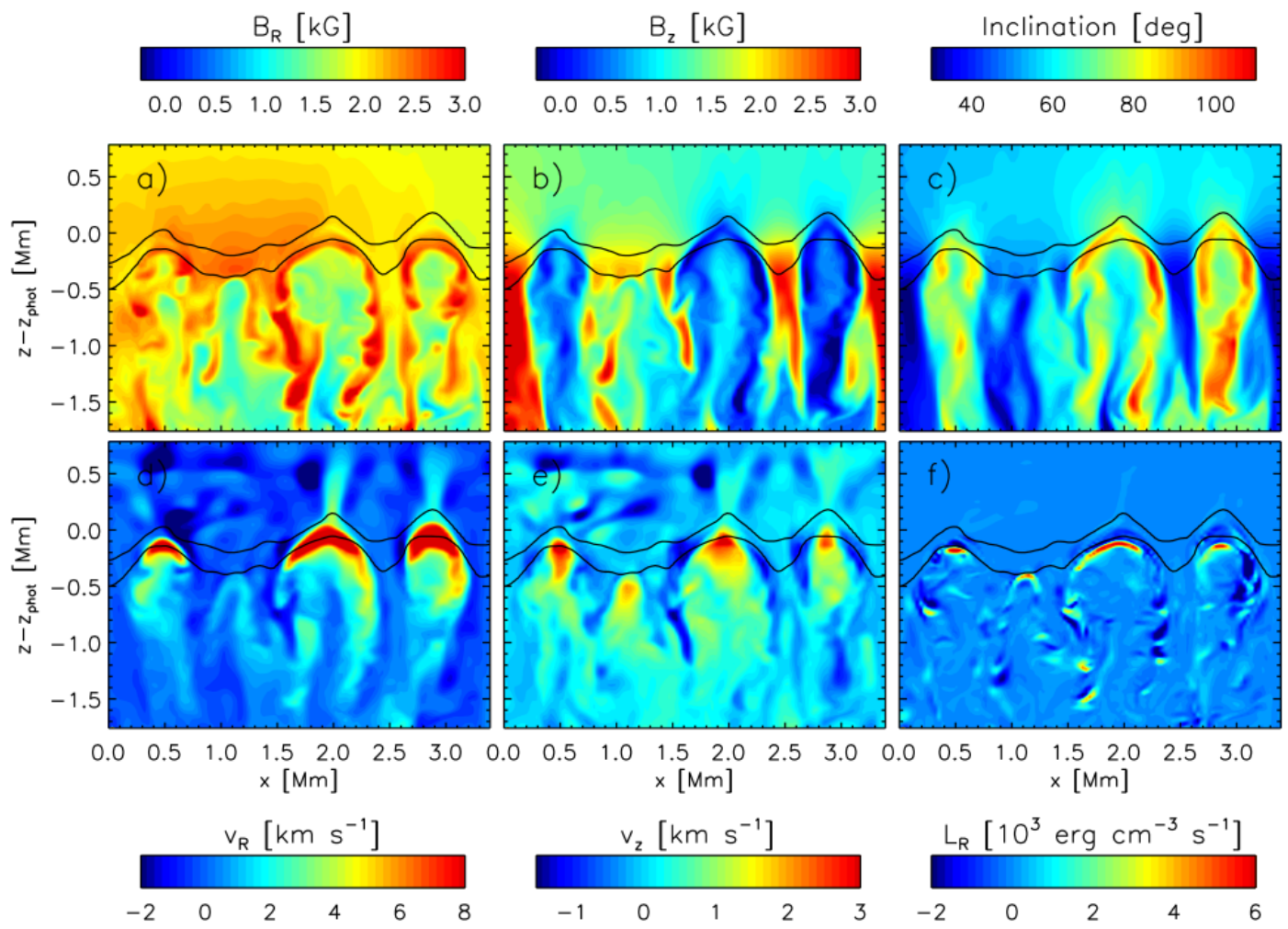

Figure 2.13: 3D MHD simulation of a sunspot by Rempel (2011): Vertical cross section of filaments in the inner penumbra. (a) radial field component, $B_{R}$, (b) vertical field component, $B_{Z}$, (c) field inclination angle, (d) radial flow velocity, $v_{R}$, (e) vertical flow velocity, $v_{Z}$, and (f) radial component of the Lorentz force, $L_{R}$. The lower and upper solid lines indicate the $\tau=1$ and $\tau=0.01$ levels, respectively. From Rempel (2011).

observed in the tails may be caused by a shock formed when the supersonically sinking gas transitions into a subsonic flow. Also, they identified a $\cap$-shaped loop-like configuration of the magnetic field. The filaments' heads and tails have more vertical fields with opposite polarities relative to each other $\left(\gamma=40^{\circ}\right.$ and $\gamma=150^{\circ}$ on average, respectively), while the field along the body of the filaments lies almost horizontally. Furthermore, they found that the tails of the filaments (all located within or near the outer edge of the penumbra) consistently display an excess magnetic field strength of $\sim 500 \mathrm{G}$ compared with the heads of the filaments, at $\tau=1$. The field in the tails reaches values of $1500-2500 \mathrm{G}$ at optical depth unity on average, and up to $3500 \mathrm{G}$ in individual tails ${ }^{1}$. Therefore, they consider the possibility of a siphon flow mechanism driving the Evershed flow, given that an enhanced field in the tails implies the existence of a gas pressure gradient driving the gas outwards. However, their analysis was performed on constant optical depth levels which, due to the strong dependency of the opacity on the temperature, may refer to different

\footnotetext{
${ }^{1}$ van Noort et al. (2013) also found that the fastest downflows in sunspot penumbrae have supersonic speeds $\left(v_{L O S} \geq 10 \mathrm{~km} \mathrm{~s}^{-1}\right)$ and occur in the tails of complex filaments (those with a single tail but with more than one head) near the penumbral periphery in regions with slightly increased temperatures compared to their surroundings, and that they are associated with strong magnetic fields $(B \sim 3 \mathrm{kG}$ at $\tau=1$ and occasionally exceeding $4 \mathrm{kG}$ ), which are almost vertical and of opposite polarity to that of the sunspot umbra.
} 
geometrical heights between heads and tails.

Schlichenmaier (2002), in his extended version of the moving flux tube model, allows the flux tube to bend back into the photosphere within the penumbra. When the system evolves into a quasi-stationary state, the magnetic field strength at the outer footpoint of the flux tube becomes larger than the field strength at the inner footpoint at the same geometrical height, therefore producing a stationary siphon flow. This is compatible with the findings of Tiwari et al. (2013). Furthermore, the supersonic downflows observed by e.g., Tiwari et al. (2013) and van Noort et al. (2013) at the filament's tails and the possible resultant shocks can be explained by considering magnetic flux tubes that have cross sections changing with height so that they can carry siphon flows that have subsonic speeds before reaching the summit of the loop, but supersonic speed after that point until it passes to subsonic through a shock. Thus, the siphon flow nature of the Evershed flow remains a plausible scenario.

The Evershed flow has also been explained as a thermally driven flow along magnetic flux tubes, i.e. driven by a form of overturning convection (see sketches shown in Fig. 2.11 and e.g., Jahn and Schmidt 1994, Schlichenmaier et al. 1998b, Schlichenmaier and Solanki 2003, Rempel 2011). In such a scenario, when the hot upflowing gas reaches the solar surface, it produces an excess of gas pressure which accelerates the gas sideways. Furthermore, the gas is also partly deflected in the radial direction by a horizontal magnetic field. Since the field inclination increases radialy outwards in the penumbra, the gas flows radially outwards along the penumbral filament. On the way, the gas cools down and sinks in the tails. Thus, the Evershed outflow is interpreted as the radial component of such a convective flow.

The results of Tiwari et al. (2013) consistently show that the filaments' heads are about 400-800 K hotter than the filaments' tails (Fig. 2.12), which is compatible with this convective picture. Furthermore, they observed a lateral flow pattern at $\tau=1$ in accordance with the convective driver scenario of the Evershed flow (downflows at the lateral edges of penumbral filaments were also observed by Esteban Pozuelo et al. 2015). These observed lateral flows are also compatible with the predictions of the field-free gap model. However, according to the proposal by Scharmer and Spruit (2006), the Evershed flow takes place in field-free gaps below the penumbral field, while the observations by Tiwari et al. (2013) confirm the strongly magnetized nature of the Evershed flow channels.

Recent 3D MHD simulations of sunspots (e.g., Heinemann et al. 2007, Rempel et al. 2009a,b, Kitiashvili et al. 2009, Rempel 2011) can reproduce many observational aspects regarding the filamentary structure of penumbrae, such as the uncombed structure of the magnetic field, the Evershed outflows along the filaments with a nearly horizontal magnetic field, and overturning convective motions. Moreover, these numerical simulations provide the opportunity to gain insight into the physical processes taking place in the penumbra on a geometrical height scale.

According to the results of a 3D radiative MHD sunspot simulation by Rempel (2011), the penumbral fine structure is a result of anisotropic overturning convection and the Evershed flow is driven by magneto-convection, that is, by vertical pressure forces in upflows that are deflected into the horizontal direction through the Lorentz force generated by the horizontal magnetic fields in the flow channels. These simulated Evershed flows can reach peak flow velocities of $8 \mathrm{~km} \mathrm{~s}^{-1}$ at $\tau=1$, and rapidly decline with height. Figure 2.13 shows a vertical cross section of some filaments in the inner penumbra from the numerical 
simulation of Rempel (2011). As pointed out therein, the enhancement of the radial field component, combined with the reduction of the vertical field component near the $\tau=1$ level, results in a strong increase of the inclination angle within a thin boundary layer near $\tau=1$. As a consequence, the positive contribution of the radial Lorentz force is enhanced near such boundary layer. The resulting outflow around $\tau=1$ are restricted to the deep photosphere and, unlike in the siphon flow scenario, the existence of an outer footpoint for each filament is a consequence of the fast outflows rather than their cause.

The convective nature of the penumbra and the Evershed flow are a common result in all radiative 3D MHD simulations to date but it is still debated in the context of observational constraints. Therefore, the implementation of more sophisticated analysis techniques to apply in future observations with improved spatial, temporal and spectral resolutions, are the key to achieve reliable measurements of vertical gradients in the magnetic field as well as in the flow velocity. Likewise, given that distinct physical processes that are important for the understanding of the penumbral configuration and its dynamics take place in the subsurface layers, it is of primary importance to continuously develop new techniques to explore the solar interior.

\subsection{Counter-Evershed flows}

Counter-Evershed flows (CEFs) are, contrary to the well-known normal Evershed flow (NEF), convergent flows (i.e., radial inflows with respect to the umbra) occurring in the deep photosphere within penumbrae ${ }^{2}$. The scarcity of observations of this type of penumbral flows is perhaps a consequence of their rare occurrence. Therefore, little is known about their nature, characteristics and their relation with the filamentary structure of penumbrae.

Frequently, the formation of a penumbra is associated with photospheric flows that have an opposite sign to that of the NEF in the photosphere (e.g., Schlichenmaier et al. 2012). Also, penumbral filaments in decaying penumbrae have been observed carrying flows that are oppositely directed to the NEF (Bellot Rubio et al. 2008). However, CEFs in well-developed penumbrae are very rare features and only a few observations have been reported (Lites et al. 2002, Kleint and Sainz Dalda 2013, Louis et al. 2014, Siu-Tapia et al. 2017).

In a first example, Lites et al. (2002) reported Doppler velocities that indicate the presence of oppositely directed Evershed flows within the penumbra of the $\delta$-sunspot ${ }^{3}$ in NOAA AR 7205 (Fig. 2.14). They studied the flow velocities and the configuration of the magnetic field within the region highlighted with a white box in Figure 2.14a (diskcenter side of the sunspot) by using observations from the Advanced Stokes Polarimeter (ASP) and by applying to these data ME least-squares inversions. Within such regions,

\footnotetext{
${ }^{2}$ Not to be confused with the inverse Evershed flow, which is the gas flow directed towards the sunspot umbra observed in the chromospheric layers along radially elongated fibrils of the superpenumbra, i.e., fibrils (assumed to form individual magnetic flux tubes) that start within the penumbra but extend well beyond the penumbra by a few sunspot radii (e.g., Loughhead 1968, Moore 1981). A siphon flow mechanism has been proposed to explain the inverse Evershed flow, with the driving force being the gas pressure difference between the two footpoints of the flux tube (Meyer and Schmidt 1968a, Maltby 1997, Thomas 1994).

${ }^{3} \delta$-configuration sunspots are spots that comprise umbrae of opposite magnetic field polarity in a single penumbra.
} 

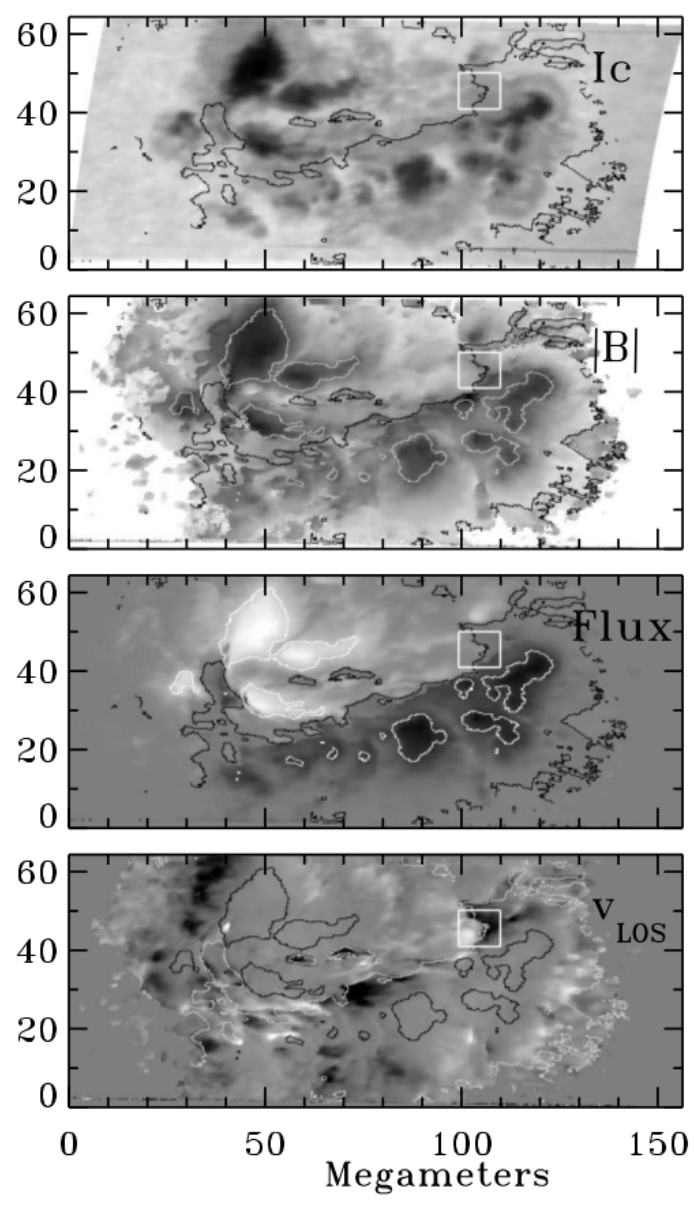

(a)

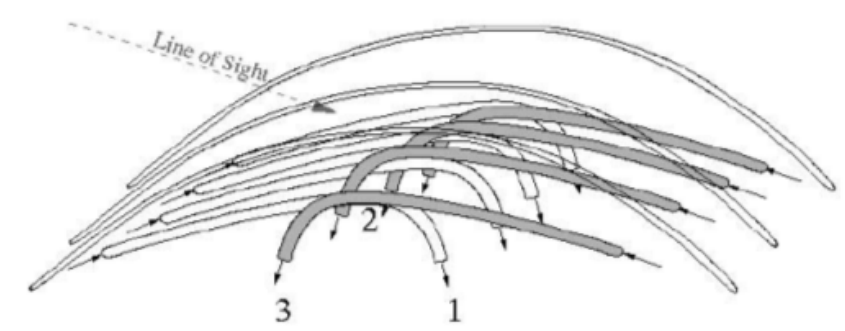

(b)

Figure 2.14: CEFs observed with the ASP in the disk-center side of the $\delta$-sunspot penumbra in NOAA AR 7205, at $\mu=0.49$ (west-limb). (a) From top to bottom: Continuum intensity image $I_{C}$, magnetic field strength $B$ (black=3000 G), signed magnetic flux (white/black $= \pm 2500 \mathrm{G}$ ), and Doppler velocity $v_{L O S}$ (white/black $= \pm 3 \mathrm{~km} \mathrm{~s}^{-1}$, positive signs indicate red-shifts), in the local solar frame, from ME least-squares inversions. The polarity inversion line (PIL) is shown in all maps, while the umbral boundaries are shown only in the three bottom maps. The region of interest is marked by white boxes. From Lites et al. (2002). (b): Sketch of the magnetic field and flow configuration near the local PIL as interpreted by Lites et al. (2002) to explain the observation of Evershed flows that converge upon the PIL. Arrows indicate the flow direction and the field orientation is from left to right. Overarching field lines are indicated by the thinner tubes. From Lites et al. (2002). 
the oppositely directed flows, $\mathrm{CEF}^{4}$ and NEF, were observed with $v_{L O S}$ of up to $+4 \mathrm{~km}$ $\mathrm{s}^{-1}$ and $-6 \mathrm{~km} \mathrm{~s}^{-1}$, respectively (positive $v_{L O S}$ indicate red-shifts). They were separated by and converging towards the local polarity inversion line (PIL). Furthermore, such a special flow configuration was observed to persist for many hours in a convex magnetic field topology, i.e. the photospheric magnetic field pointed towards the PIL on the positive polarity side, and away from the PIL on the negative polarity side. The authors also performed an analysis based on two-component ME inversions, whose results suggest large flow speeds (comparable to the sound velocity at some locations: $\sim 6-8 \mathrm{~km} \mathrm{~s}^{-1}$ ) and the possibility of two atmospheric components with oppositely directed flows present within a resolution element of $\approx 1^{\prime \prime}$. Based on their results, the authors proposed a scenario in which the convergent flows have their origin in the typical Evershed effect, which occurs along penumbral filaments that come together from different directions and meet near the PIL (see Fig. 2.14b). In Figure 2.14b, the dark filaments represent the penumbral filaments carrying the NEF within the region of interest and the white filaments carry the $\mathrm{CEF}$, while the field lines that reach higher levels in the atmosphere (thinner tubes) form a canopy above the convergence zone and do not carry any Evershed flow. Thus, Lites et al. (2002) suggest that the oppositely directed Evershed flows are both NEFs that emanate from opposite polarity umbrae and return to the solar interior near the convergence zone, where the small-scale flow pattern is presumed to be largely unresolved in their observations.

In a second study, an anomaly in the flow pattern of a sunspot penumbra was reported by Kleint and Sainz Dalda (2013). They found at least three unusual penumbral filaments penetrating the umbra during the evolution of AR 11302, displaying a typical penumbral brightness intensity but being atypically fainter towards their heads. Those unusual filaments were found to be wider (by a factor of 2-3) than the typical penumbral filaments, with a hook-shaped configuration, and carrying photospheric CEFs (Fig. 2.15). According to Kleint and Sainz Dalda (2013), these unusual filaments have lifetimes that range from several hours to a few days and their dynamic evolution revealed that these structures could lead to flares. To study the magnetic field and the flow configuration along those unusual filaments, Kleint and Sainz Dalda (2013) performed SIR inversions (Ruiz Cobo and del Toro Iniesta 1992) of the relevant Hinode SOT/SP data. They used a twocomponent model with different initial conditions in the LOS field inclination and field strength. They referred to as model 1 to the more horizontal and weaker magnetic field component which is related to the penumbral filaments (middle row in Fig. 2.15) and asmodel 2 to the more vertical and stronger field component (bottom row in Fig. 2.15). As described in Kleint and Sainz Dalda (2013), two unusual filaments are observed in the lower and upper part of the umbra in the analyzed scan. In particular, the Evershed flow was found to move inwards from the outer part of the penumbra to the umbra along both filaments. According to their two-component model, the magnetic field in the unusual filaments is stronger than the penumbral fields in the sunspot. Moreover, the field inclination along the two filaments goes from almost vertical in the inner penumbra to almost horizontal in the outer penumbra, although more vertical fields were observed at their footpoints.

To explain the topology of the unusual filaments, Kleint and Sainz Dalda (2013) pro-

\footnotetext{
${ }^{4}$ Referred to as receding flows in Lites et al. (2002).
} 

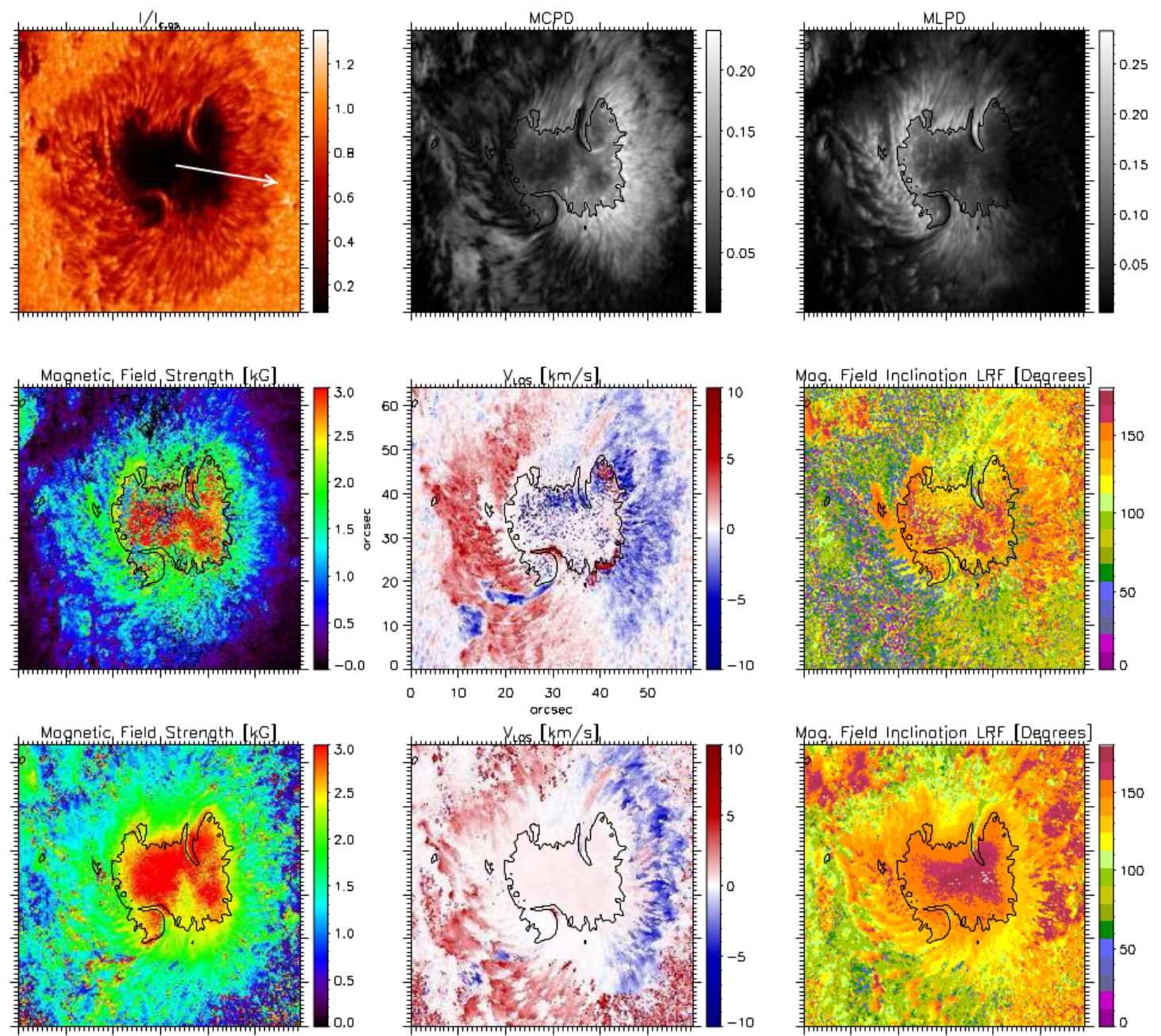

Figure 2.15: CEFs observed with Hinode SOT/SP on September 2011 along two unusual filaments that penetrate into the umbra of the sunspot in AR 11302, at $\mu=0.66$ (Kleint and Sainz Dalda 2013). Top row: intensity normalized to the continuum quiet-sun intensity, circular, and linear polarization degree. Middle row: magnetic field strength (kG), LOS velocity $\left(\mathrm{km} \mathrm{s}^{-1}\right)$, and magnetic field inclination in the local reference frame $\left(^{\circ}\right)$ from SIR inversions for model 1 (see text). Bottom row: Results of SIR inversions for model 2 (see text), in the same format as the plots in the middle row. The white arrow on the intensity map points towards the disk center. Black contours show the umbra-penumbra boundary. From Kleint and Sainz Dalda (2013). 

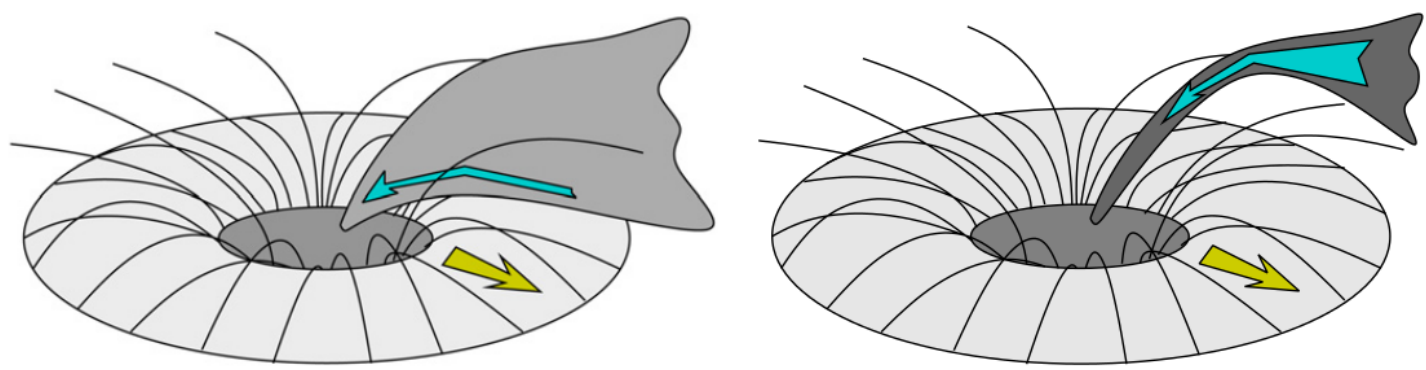

Figure 2.16: Two schematic scenarios proposed by Kleint and Sainz Dalda (2013) to explain the topology of unusual filaments observed carrying CEFs: the umbral filament sheet model (left) and the massive umbral filament model (right). The lines that appear to connect the outer part of the umbra with the outer part of the penumbra represent the more horizontal penumbral field component, while the more vertical component is drawn as unconnected lines that start in the outer part of the umbra. the yellow arrows indicate the direction of the NEF, while the cyan arrows represent the CEF. From Kleint and Sainz Dalda (2013).

posed two scenarios: the umbral filament sheet model and the massive umbral filament model (Fig. 2.16). In the first model, the topology of the unusual filaments is described as a two-dimensional sheet that connects the umbra with a region located well outside the sunspot, e.g., in the network (see left panel in Fig. 2.16). Thus, under such a scenario, a gas pressure difference between the base of the sheet inside and outside the umbra produce a siphon flow in which the gas flows inwards along the sheet. In the second model, the authors propose that the unusual filaments are thick magnetic flux tubes carrying the chromospheric inverse Evershed flow (see right panel in Fig. 2.16). A high density of the inflowing gas would avoid the NEF from being observed since it would produce an elevation of the $\tau=1$ surface. Finally, Kleint and Sainz Dalda (2013) argue that both scenarios can explain the visibility of the unusual filaments in different atmospheric layers, but the data did not allow them to determine if the inflows were present at all heights. Hence, they could not exclude either of them.

A third set of possible observations of photospheric CEFs was presented by Louis et al. (2014). They reported a blue-shifted filamentary structure on the limb-side penumbra of the leading sunspot of NOAA AR 11271 that persisted for almost two hours, and analyzed it by using MERLIN inversions (Lites et al. 2007) of Hinode SOT/SP data. According to their results (Fig. 2.17), the blue-shifted filament had maximum blue-shifts corresponding to LOS velocities of $1.6 \mathrm{~km} \mathrm{~s}^{-1}$, with a width of $1^{\prime \prime}$ and a length of $5^{\prime \prime}$. The magnetic field inclination in the blue-shifted filament was found to vary between $95^{\circ}$ and $100^{\circ}$ and the field strength between $700 \mathrm{G}$ and $1500 \mathrm{G}$ (decreasing with radial distance to the umbra). Red-shifted features corresponding to the NEF were also observed adjacent to the blue-shifted filament, all of them radially oriented in the penumbra. Furthermore, intense chromospheric activity was found to be temporally and spatially related to the photospheric blue-shifted filament. Louis et al. (2014) proposed a scenario in which the analyzed blue-shifted filament contains photospheric gas upflows which transport the magnetic field towards the upper atmospheric layers. This could then lead to magnetic reconnection with the overlying magnetic field of the sunspot and therefore, produce intense 

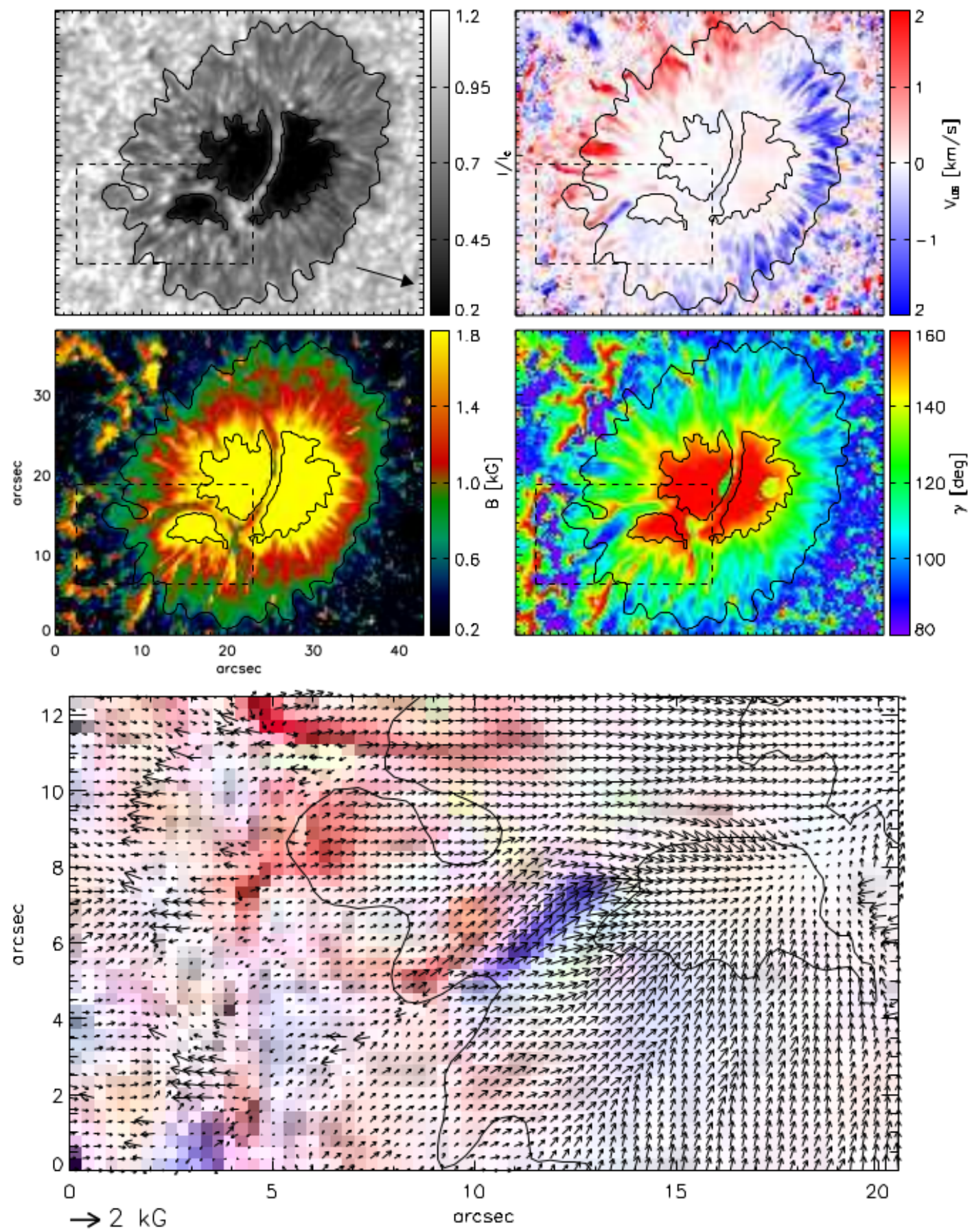

Figure 2.17: CEFs observed with Hinode SOT/SP in the leading sunspot of NOAA AR 11271, at $\theta=29^{\circ}$ (Louis et al. 2014). Upper panel: (clockwise) the maps show the Hinode G-band intensity image and the results from a MERLIN inversion: the LOS velocity, the magnetic field inclination in the local reference frame and the field strength. The arrow on the intensity map points towards the disk center. Bottom panel: Close up of the LOS velocity map of the region highlighted by dashed boxes in the upper panel maps. The arrows indicate the horizontal magnetic field component in the LRF. From Louis et al. (2014). 
chromospheric brightening. However, the authors did not discard a scenario in which the blue-shifts in the analyzed filament were produced by radial inflows of plasma in the photospheric penumbra (i.e. CEFs). Based on the numerical results of Botha et al. (2011), who studied non-linear convective instabilities around a central cylindrical magnetic flux tube and found that the stability of the central tube depends on the convection around it and, furthermore, that magnetic flux can be added to the central tube when flux caught in the surrounding convection is pushed towards the tube, Louis et al. (2014) suggested that CEFs can be a result of plasma moving towards the umbra when magnetic flux is pushed and added to the sunspot by the surrounding convective flows.

Finally, the most prominent photospheric CEFs heretofore observed were reported by Siu-Tapia et al. (2017) and will be described in detail in Chapter 3. Therein, we present our analysis of the flow characteristics and the fine structure of the large penumbral sector harboring the CEFs on the disk-center side penumbra of the main sunspot in NOAA AR 10930 and compare them with the well-known NEF. In addition, in Chapter 4, we present an in-depth analysis of a high-resolution magnetohydrodynamical simulation of a sunspot (Rempel 2015) whose penumbra harbors localized CEFs at photospheric heights. Hitherto, these simulations represent a unique opportunity to explore the underlying physics of CEFs on a geometrical height scale and, moreover, to look into vertical gradients at subphotospheric depths which are necessary to determine the dominant driving forces of the CEFs and to compare them with the forces driving the NEFs. 


\section{Normal and counter Evershed flows in the photospheric penumbra of a sunspot: SPINOR 2D inversions of Hinode-SOT/ SP observations}

The contents of this chapter are identical to the printed version of A. Siu-Tapia, A. Lagg, S. K. Solanki, M. van Noort and J. Jurčák, A\&A, vol. 607, page A36, 2017, reproduced with permission (CESO.

Context. The Evershed effect, a nearly horizontal outflow of material seen in the penumbrae of sunspots in the photospheric layers, is a common characteristic of well-developed penumbrae, but is still not well understood. Even less is known about photospheric horizontal inflows in the penumbra, also known as counter Evershed flows.

Aims. Here we present a rare feature observed in the penumbra of the main sunspot of AR NOAA 10930. This spot displays the normal Evershed outflow in most of the penumbra, but harbors a fast photospheric inflow of material over a large sector of the disk-center penumbra. We investigate the driving forces of both, the normal and the counter Evershed flows.

Methods. We invert the spectropolarimetric data from Hinode SOT/SP using the spatially coupled version of the SPINOR inversion code, which allows us to derive heightdependent maps of the relevant physical parameters in the sunspot. These maps show considerable fine structure. Similarities and differences between the normal Evershed outflow and the counter Evershed flow are investigated.

Results. In both the normal and the counter Evershed flows, the material flows from regions with field strengths of the order of $1.5-2 \mathrm{kG}$ to regions with stronger fields. The sources and sinks of both penumbral flows display opposite field polarities, with the sinks (tails of filaments) harboring local enhancements in temperature, which are nonetheless colder than their sources (heads of filaments).

Conclusions. The anti-correlation of the gradients in the temperature and magnetic pressure between the endpoints of the filaments from the two distinct penumbral regions is compatible with both the convective driver and the siphon flow scenarios. A geometrical scale of the parameters is necessary to determine which is the dominant force driving the flows. 
3 Normal and counter Evershed flows in the photospheric penumbra of a sunspot: SPINOR 2D inversions of Hinode-SOT/ SP observations

\subsection{Introduction}

The penumbrae of sunspots are strongly magnetized media (with field strengths of $\sim 1$ to $2 \mathrm{kG}$ ) where convection is expected to be almost completely suppressed according to simple estimates (Biermann 1941, Cowling 1953, Meyer et al. 1974, Jahn and Schmidt 1994). Consequently, the penumbral brightness is expected to be much lower than the observed one, which is $\sim 75-80 \%$ that of the quiet Sun, integrated over wavelength. This fact points towards some level of convection taking place in the penumbra to account for its observed brightness. However, how the energy is transported in penumbrae is still one of the major open questions in solar physics. Detailed reviews pointing out this open problem and providing discussions on some proposed solutions have been given by, for example, Solanki (2003), Thomas and Weiss (2004, 2008), Borrero (2009), Scharmer (2009), Schlichenmaier (2009), Tritschler (2009), Bellot Rubio (2010), Borrero and Ichimoto (2011), Rempel and Schlichenmaier (2011).

The filamentary structure of the penumbra might provide one of the main clues helping us to gain insight into this subject. The penumbral magnetic field consists of two major components: spines, seen as relatively dark regions where the magnetic field is stronger and more vertical, and intraspines/filaments, seen as elongated bright channels where the magnetic field is weaker and more horizontal (see review by Borrero and Ichimoto 2011).

Various models have been proposed to account for the filamentary structure of penumbrae. One of these, the embedded flux tube model, is empirical in nature and was proposed by Solanki and Montavon (1993). This model mainly describes the complex threedimensional (3D) structure of the field to explain asymmetric Stokes $V$ profiles, and points to nearly horizontal magnetic flux tubes forming intraspines that are embedded in more vertical background magnetic fields (spines) in penumbrae. The downward pumping mechanism (Thomas et al. 2002) was proposed to explain the origin of field lines that return to the solar surface at the outer penumbra. Another idea to account for the penumbral filaments is the field-free gap model (Choudhuri 1986, Scharmer and Spruit 2006, Spruit and Scharmer 2006), where the penumbral bright filaments are described as regions where the vertical component of the magnetic field vanishes as a result of the interaction with the non-magnetized gas that rises due to convection into a background with more oblique fields.

These models concentrate on the configuration of the magnetic field in the penumbra. However, the appearance of a penumbra is always associated with a distinctive gas flow, that is, the Evershed flow (EF; Evershed 1909) and, therefore, this must also be taken into account by these models. The $\mathrm{EF}$ is the most prominent dynamic phenomenon in sunspots: An outward-directed flow observed in the photospheric layers of penumbrae with speeds of several $\mathrm{km} \mathrm{s}^{-1}$ and large inclinations to the vertical. This phenomenon is thought to be closely related to the filamentary structure of penumbrae (e.g., Borrero and Ichimoto 2011) and given its ubiquity, it is expected to play a central role in the energy transport in the penumbrae of sunspots.

The EF is usually observed as a blueshift of photospheric spectral lines in the diskcenter-side part of the penumbra and a corresponding redshift in the limb-side part of the penumbra. This is generally interpreted as a radial, nearly horizontal outflow of matter. The EF is height dependent: in the photosphere the line shifts decrease rapidly with height of line formation (St. John 1913, Maltby 1964, Börner and Kneer 1992). Moreover, in 
the chromosphere the line shifts change sign (inverse EF), with the center-side part of the penumbra now showing redshifts (St. John 1913, Börner and Kneer 1992, Tsiropoula 2000). This is taken to be the signature of an inflow of material. Most of the mass flowing outwards in the photosphere returns to the solar interior within the penumbra, in opposite polarity downflow channels (Westendorp Plaza et al. 1997, 2001a), although a part of the Evershed flow continues in the canopy of the sunspot (Solanki et al. 1994).

The origin and driving physical mechanisms of the EF have been the subject of considerable controversy for decades. Some models describe it as a siphon flow driven by a gas pressure difference between the footpoints of arched magnetic flux tubes (Meyer and Schmidt 1968a, Thomas and Weiss 1992, Montesinos and Thomas 1997, Thomas et al. 2006); while, in others, the EF is explained as a flow along magnetic flux tubes driven by a form of convection (Jahn and Schmidt 1994, Schlichenmaier et al. 1998a, Schlichenmaier and Solanki 2003). According to a more recent proposal by Scharmer and Spruit (2006), the EF takes place in field-free gaps below the penumbral field.

Recent 3D magnetohydrodynamical (MHD) simulations for penumbral fine structure (Heinemann et al. 2007, Rempel et al. 2009a,b, Rempel and Schlichenmaier 2011, Rempel 2011, 2012b) display a flow very similar to the EF. In such simulations the EF has typically been interpreted to be a consequence of overturning convection: the hot gas rising from below the surface is deflected by the inclined magnetic field of the penumbra, producing a fast flow toward the sunspot border. Part of the rising gas turns over laterally and dips down below the solar surface. The convective cells are then elongated in the preferred direction imposed by the magnetic field (the radial direction), forming penumbral filaments with a fast Evershed outflow along their axes and weaker downflows towards their sides, much in the same way as in quiet-sun granules.

The relative importance of various forces for driving the EF is still a matter of debate. The reason is that along a filament harboring a flow, both the temperature and the magnetic field show the correct sign of the gradient along the filament (Tiwari et al. 2013).

In this paper, we report and study the characteristics of an atypical photospheric inflow observed by Hinode SOT/SP over a considerable sector of the penumbra in the main sunspot of NOAA AR 10930. Observations of photospheric counter Evershed flows are rarely reported, and are usually restricted to very narrow channels (see, e.g., Kleint and Sainz Dalda 2013, Louis et al. 2014), or are transient during the formation phase of the penumbra, (see e.g., Schlichenmaier et al. 2012, Romano et al. 2014, Murabito et al. 2016). The observation of such a large inflowing region in a fully developed penumbra is, to our knowledge, unique. By comparing the properties of the anomalous counter EF with the well-known photospheric Evershed outflow, we hope to learn more about the drivers of both flows.

This work is organized as follows: In Section 3.2 we describe our data and inversion technique. In Section 3.3, results are presented, which are then discussed in Section 3.4. Finally, in Section 3.5 we draw our conclusions. 


\subsection{Observational data and analysis techniques}

\subsubsection{Observations}

For our study, we utilize spectropolarimetric observations in the Fe I 6301.5 and 6302.5 $\AA$ lines from the Hinode spectropolarimeter of the Solar Optical Telescope (SOT/SP) (Kosugi et al. 2007, Lites 2007, Ichimoto et al. 2008, Lites et al. 2013) of the main sunspot of the active region (AR) NOAA 10930 on December 08, 2006 (see Fig. 3.1). The SOT/SP provided us with a full-Stokes dataset with a spatial sampling of $\sim 0$ " $.16 /$ pixel while operating in normal mode (see Lites et al. (2013) for a detailed description of the SP instrument).

The sunspot umbra displays a negative magnetic polarity. It was observed at (S91", E698'), that is, at a heliocentric angle $\theta \approx 47^{\circ}$. The observations were reduced with the corresponding routines of the Solar-Soft package (Lites and Ichimoto 2013).

\subsubsection{Inversions}

We infer the atmospheric properties of the sunspot by inverting the observational data with the spatially coupled version (van Noort 2012) of the SPINOR inversion code (Frutiger et al. 2000) which uses the STOPRO routines (Solanki et al. 1987) to solve the radiativetransfer equations for polarized light under the assumption of local thermodynamic equilibrium (LTE).

The inversion code calculates the best fit full-Stokes spectra of an atmospheric model described by a selected number of atmospheric parameters, specified at a number of optical depth positions, and interpolated using a bicubic spline approximation. The spatially coupled version is able to invert the observed data while taking into account the spatial degradation introduced by the telescope diffraction pattern. Simultaneously it keeps the atmospheric model as simple as possible. The atmospheric parameters returned by the code provide the best fits to the Stokes profiles in the absence of the blurring effect of telescope diffraction.

The inversion is performed on a denser spatial grid than that of the original data. The resulting inverted parameters have a significantly improved spatial resolution in most fits and appear to produce a more robust inversion result than at the original pixel size (van Noort et al. 2013). Here we use a pixel size of $0^{\prime \prime} .08 /$ pixel, a factor 2 smaller than in the original data, allowing structures down to the diffraction limit of the telescope to be adequately resolved. The returned parameters show small-scale structures sharper than in the original data to the extent allowed by noise (see van Noort (2012), van Noort et al. (2013) for details and a discussion of the results of equivalent inversions of similar Hinode/SP data).

The inverted parameters are temperature $T$, magnetic field strength $B$, field inclination relative to the line-of-sight $\gamma_{L O S}$, field azimuth $\phi$, line-of-sight velocity $v_{L O S}$, and a micro-turbulent velocity $v_{M I C}$. All the free parameters were allowed to vary at all three height nodes, which were placed at $\log (\tau)=0.0,-0.8$ and -2.0 , respectively. The stratifications are then extrapolated linearly above $\log (\tau)=-2.0$ up to $\log (\tau)=-4.0$, and below $\log (\tau)=0$ down to $\log (\tau) \sim 1.3$

The inversion returns very large field strengths, in excess of $7 \mathrm{kG}$, in about 200 pixels 
located near the umbra/penumbra boundary of the center-side penumbra (see e.g. yellow markers on Fig. 3.5b). Figure 3.2d shows the observed Stokes profiles (dashed lines) in one of those pixels (yellow marker on the maps of Fig. 3.1). These profiles are highly complex since they exhibit large asymmetries and multi-lobed Stokes $V$ profiles, which causes their best-fits from the inversion to be not nearly as good as in most of the penumbral pixels. The SPINOR 2D best-fits to these profiles (solid red lines) feature very large line-of-sight velocities and magnetic field strengths at all three height nodes in order to reproduce the large wavelength separation in terms of the Zeeman splitting: $v_{L O S} \sim 9.3$ $\mathrm{km} \mathrm{s}^{-1}$ and $B \sim 8.3 \mathrm{kG}$ at $\log (\tau)=0$.

These unusually strong penumbral magnetic fields are not new, as they have been previously observed in supersonic penumbral downflows (van Noort et al. 2013). However, they show up only when a spatially coupled inversion is performed and their reality needs to be confirmed with other techniques ${ }^{1}$. To examine the reliability of the inversions in the pixels with very large field strengths is not the main aim of this work. We instead exclude in the present analysis all those pixels where the inversion gives $B>7 \mathrm{kG}$.

Also, it is an intrinsic problem of inversions to specify errors in the fitted atmospheric parameters. Especially in the case of the 2D coupled inversions, the changes in the parameters of a single pixel severely affect the result, and therefore the error, of the neighboring pixels. This fact makes the computation of formal errors for a single pixel impossible. We stress that the main error of inversions is not the formal error in one pixel resulting from the minimization procedure, but is introduced by the choice of the correct model atmosphere. The best error estimate can therefore be provided by comparing the results from different model atmospheres. Such an analysis is beyond the scope of this work and will be presented in detail in another publication. Preliminary results of this analysis indicate that strong magnetic field values of $5 \mathrm{kG}$ and even $\sim 6 \mathrm{kG}$ represent a valid solution for the fit to the Stokes profiles in multiple models, and that models returning magnetic fields larger than $7 \mathrm{kG}$ provide the best fit to the observed profiles.

Finally, after the inversion, the $180^{\circ}$ azimuthal ambiguity was resolved using the NonPotential Magnetic Field Computation method (NPFC; Georgoulis 2005), which determines the non-potential component of the field to minimize the vertical current density $J_{z}$. The NPFC code also converts the values of magnetic field inclination and azimuth from the line-of-sight (LOS) frame into the local reference frame (LRF). This step helps to determine the correct inclination and azimuth of the field in the LRF and is helpful for interpreting our results. However, we are aware that disambiguation techniques may not give reliable results at the small scales studied in this paper. Some of the reasons why such techniques may fail are as follows: 1) The smallest structures we are studying have a horizontal dimension that is similar to the vertical corrugation of the $\tau=1$ surface. This invalidates the general assumption of disambiguation techniques that the field was measured on a flat surface. 2) Electric currents (e.g., current sheets at boundaries of filaments) are likely not properly resolved and thus may be underestimated. This may influence the results of techniques that aim to minimize the non-potential part of the field, such as the technique of Georgoulis (2005). 3) In the highly dynamic environment of the penumbra, with its waves, supersonic flows, chromospheric jets, and so on, it is not clear if a minimization of the current really makes sense at the scales we are studying in the

\footnotetext{
${ }^{1}$ See Chapter 5.
} 


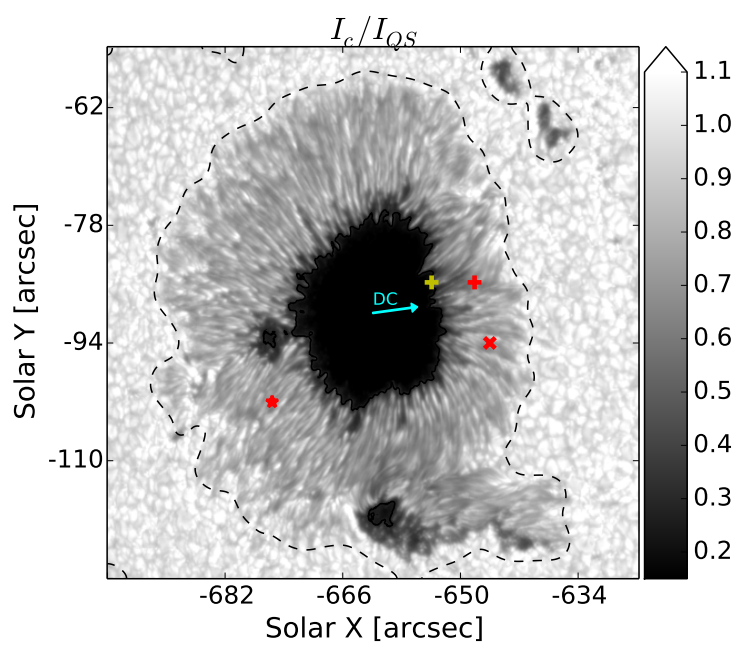

(a)

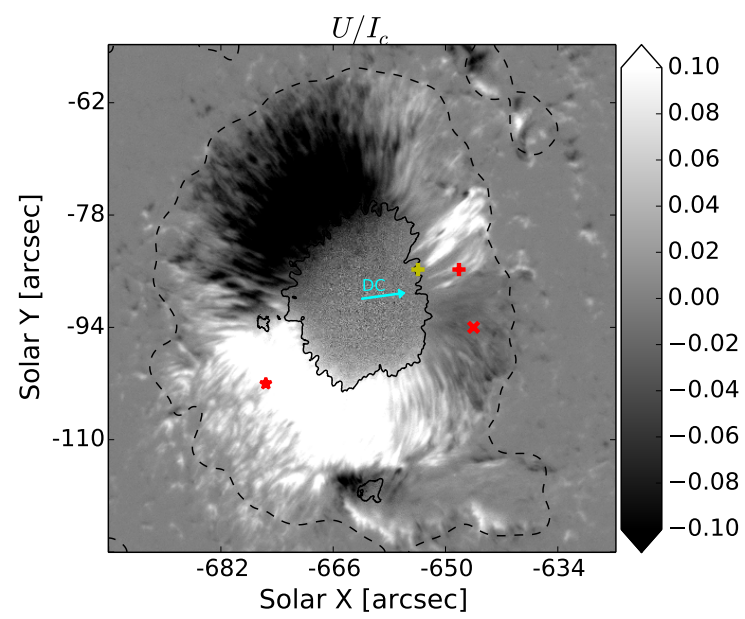

(c)

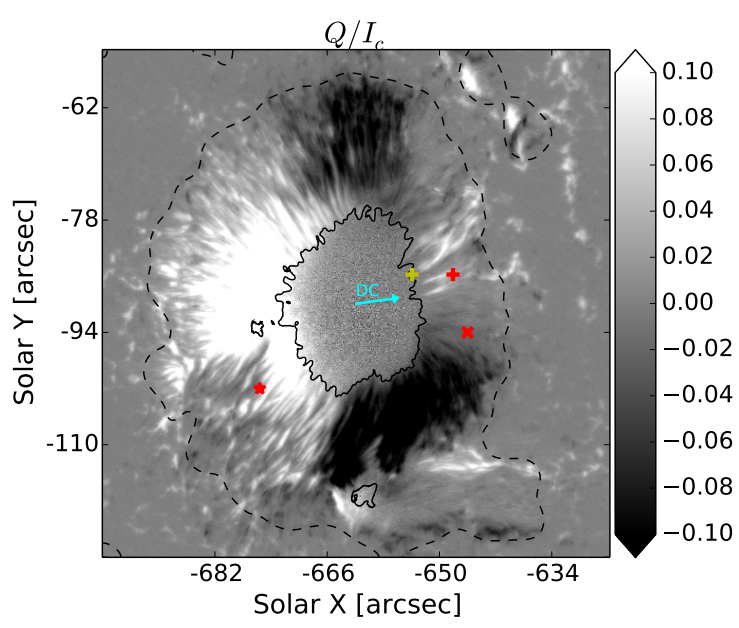

(b)

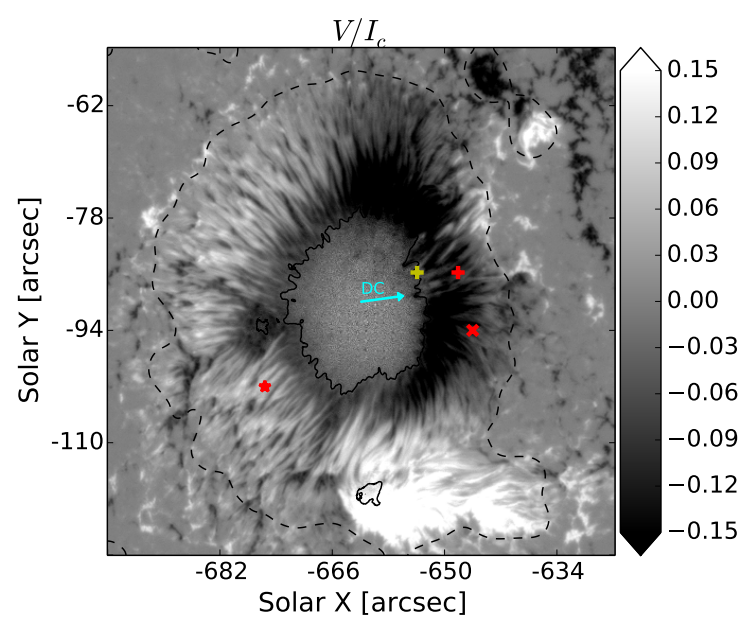

(d)

Figure 3.1: Stokes maps observed by the Hinode SOT/SP in the main sunspot of the NOAA AR 10930 on December 08, 2006. Panel (a) shows the continuum intensity $I_{c}$ normalized to the mean continuum value in the quiet sun, $I_{Q S}$. Panels (b), (c), and (d) show the maps of the Stokes parameters $Q, U$, and $V$, respectively, normalized to local $I_{c}$. The Stokes $Q, U$ and $V$ maps were constructed at $-0.1 \AA$ from the $6302.5 \AA$ line core (the selected wavelength is indicated by vertical green lines in Fig. 3.2). The umbra-penumbra boundary (black solid contour) was placed at $I_{c} / I_{Q S}=0.26$ and the external penumbral boundary (black dashed contour) is at $I_{c} / I_{Q S}=0.94$. The cyan arrows point towards the disk center. Red markers show three selected pixels, one located in the limb-side penumbra ('*'), and two located in the center-side penumbra (' $x$ ' and ' + ', respectively); their corresponding Stokes profiles are shown in Figures 3.2a, 3.2b, and 3.2c, respectively. The yellow cross shows a pixel close to the inner penumbral boundary where the inversions give $B>7 \mathrm{kG}$ at $\log (\tau)=0$ and whose Stokes profiles are shown in Figure 3.2d.

photosphere. These caveats must be borne in mind when considering the results in the LRF. 


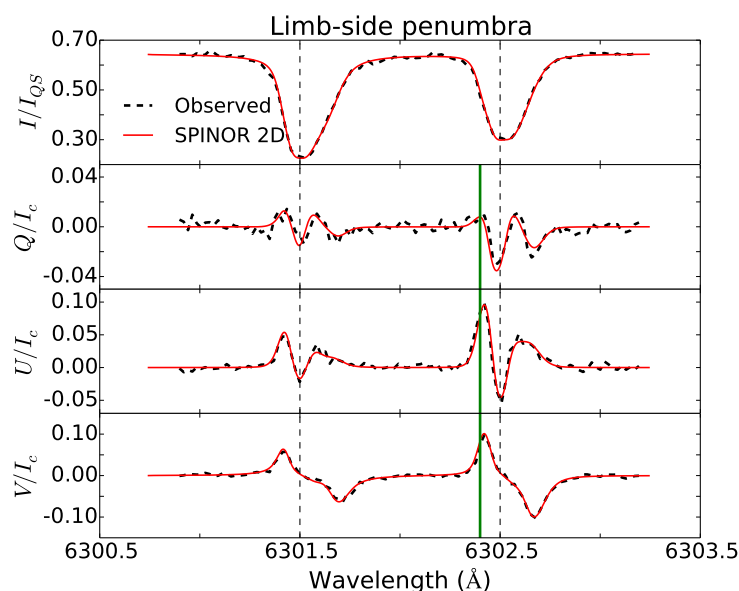

(a)

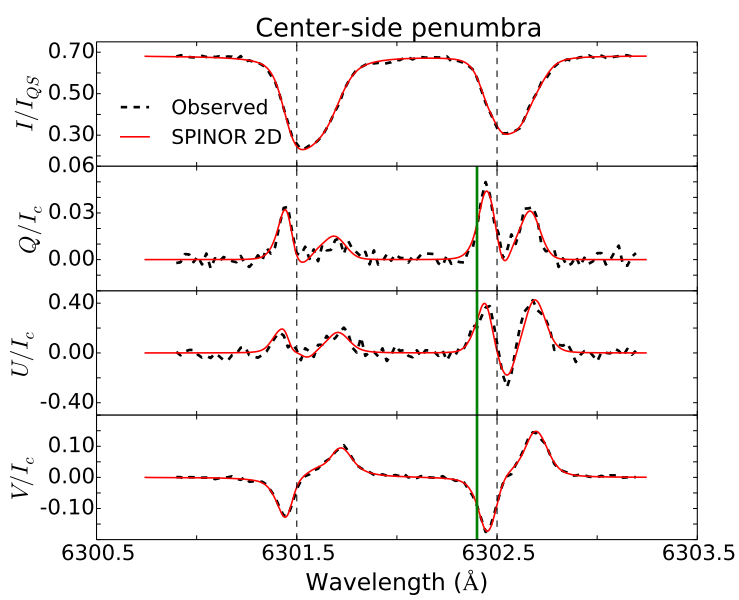

(c)

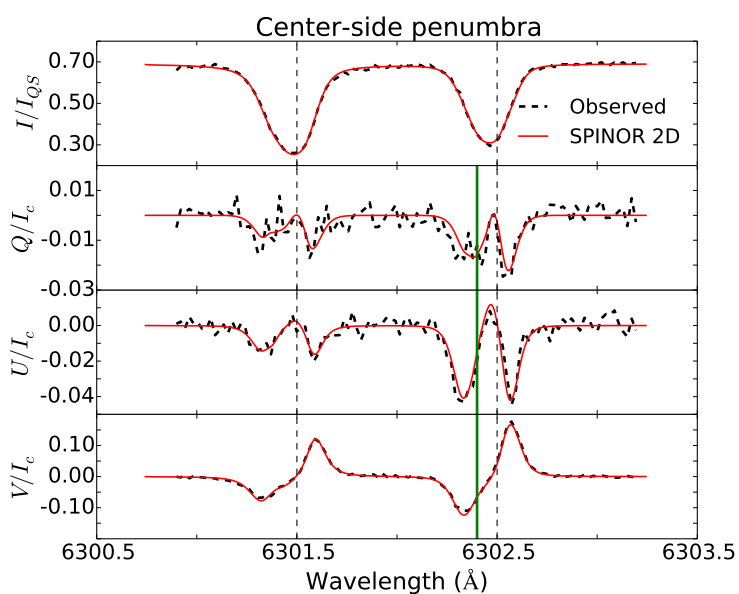

(b)

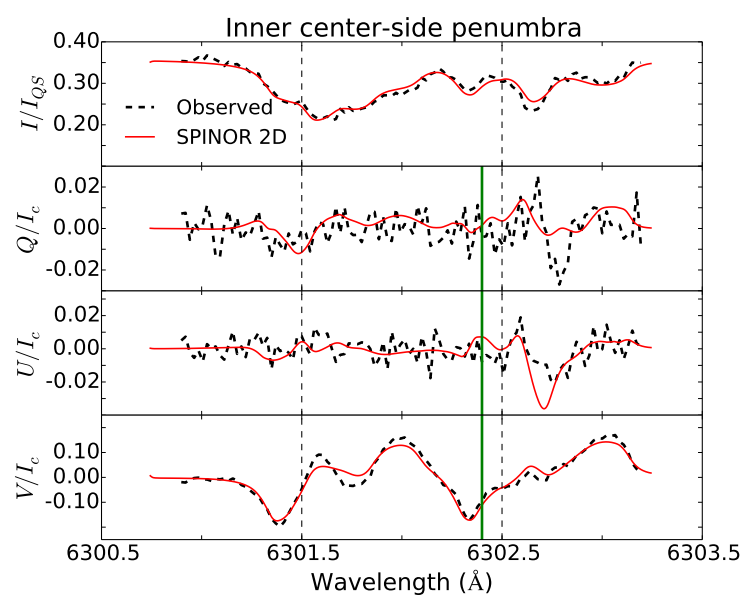

(d)

Figure 3.2: Observed Stokes profiles (dashed lines) and SPINOR 2D best-fits (solid red lines), in the wavelength interval $6300.90-6303.19 \AA$, at the location of the red markers shown in Fig. 3.1: (a) From top to bottom: Stokes $I / I_{Q S}, Q / I_{c}, U / I_{c}$ and $V / I_{c}$ in a pixel from the limb-side penumbra (red ' $*$ ' in Figure 3.1); vertical green lines in the blue wing of $6302.5 \AA$ indicate the selected wavelength used to construct the Stokes $Q / I_{c}, U / I_{c}$, and $V / I_{c}$ maps in Figure 3.1 ( $-0.1 \AA$ from $6302.5 \AA$ line core). Vertical dashed lines were placed at $6301.5 \AA$ and at $6302.5 \AA$, respectively. (b), (c), and (d) show the Stokes profiles in three pixels from the center-side penumbra (red ' $x$ ', red ' + ' and yellow ' + ' in Fig. 3.1, respectively) in the same format as in (a). The SPINOR 2D best fits of profiles in panel (d) give $B \sim 6.4,8.0$ and $8.3 \mathrm{kG}, \gamma \sim 141,148$ and $145^{\circ}$, and $v_{L O S} \sim 5.6,8.3$ and $9.3 \mathrm{~km}$ $\mathrm{s}^{-1}$ at $\log (\tau)=-2.0,-0.8$ and 0 , respectively, with $\chi^{2}=14$.

\subsection{Results}

In Figure 3.1, we show normalized maps of the Stokes parameters in the sunspot from a single SOT/SP scan. Except for the additional umbral-like feature in the bottom part of the maps, and its corresponding penumbral-like extension (S olar $X \approx\left[-666^{\prime \prime},-634^{\prime \prime}\right]$, Solar $\left.Y \approx\left[-126^{\prime \prime},-115^{\prime \prime}\right]\right)$, which we do not study in this work, the well-developed 
3 Normal and counter Evershed flows in the photospheric penumbra of a sunspot: SPINOR 2D inversions of Hinode-SOT/ SP observations

penumbra surrounding the main umbra of this sunspot seems, at first sight, quite normal; it shows quasi-radial filamentary structures all around the sunspot, and is observed within a range of continuum intensities of $26-94 \%$ of that of the average quiet Sun.

However, a more careful inspection through the individual Stokes profiles at different places within the penumbra reveals an anomalous aspect: while it is possible to observe the normal EF (NEF), that is, the photospheric absorption lines over the limb-side and the disk-center-side penumbrae are redshifted and blueshifted, respectively, indicating motions away from and towards the observer (see, e.g., Stokes profiles in Figs. 3.2a and $3.2 \mathrm{~b}$, respectively), strong redshifts are also observed over a broad portion of the centerside penumbra (see, e.g., Stokes profiles in Fig. 3.2c), indicating a counter EF (CEF).

The existence of the CEF in the center-side penumbra becomes clearer after the inversions, since most of the observed line profiles from the center-side penumbra show the same magnetic polarity (i.e., same signs of the Stokes $V$ profiles) and differ from those in the NEF region only in more subtle ways, such as in the line shifts and asymmetries. Nonetheless, the Stokes maps in Figures 3.1a, 3.1b and 3.1c suggest that the penumbral filaments in the CEF region have a slightly different orientation (not completely radially oriented) compared to that in the other, undisturbed parts of the penumbra (which contain more radially oriented filaments).

Figure 3.3 shows some of the resulting maps of the physical parameters inverted with SPINOR 2D at three continuum optical depth levels, $\log (\tau)=-2.0,-0.8$ and 0 , displayed from left to right. From the $v_{L O S}$ maps (third row), it is possible to observe the NEF, that is, the limb-side and center-side penumbrae are redshifted and blueshifted, respectively. Additionally, as indicated by the black thick contours, the CEF is observed over a rather large area of the center-side penumbra, which mostly contains positive $v_{L O S}$ values indicating motions away from the observer. Such motions, because of their center-side location close to the symmetry line (line connecting the sunspot's center with the diskcenter), represent either downward motions of material or inflows directed from the outer penumbra towards the umbra of the sunspot.

Both the NEF and the CEF are observed at all three selected atmospheric layers. The largest blueshifts are observed at the deepest observable layer $(\log (\tau)=0)$ in the centerside NEF penumbra, with associated velocities (in the line-of-sight direction) that reach values of up to $v_{L O S} \sim-15 \mathrm{~km} \mathrm{~s}^{-1}$. The largest redshifts are located at the innermost part of the CEF penumbra (towards the umbra-penumbra boundary). These redshifts also become stronger with depth, corresponding to extreme velocities $\left(v_{L O S}>20 \mathrm{~km} \mathrm{~s}^{-1}\right)$ and exceeding even the ones found by van Noort et al. (2013) in peripheral downflows within sunspot penumbrae $\left(v_{L O S} \sim 22 \mathrm{~km} \mathrm{~s}^{-1}\right)$.

The inverted maps in Figure 3.3 do not show a clear difference in temperature between the part of the penumbra showing a NEF and the one harboring the CEF, in any of the three atmospheric layers. However, at all three layers, the magnetic field strength appears to be larger at the inner part of the CEF penumbra than in any part of the NEF penumbra. Moreover, the inversions give values $B>5 \mathrm{kG}$ in some regions located in the innermost part of the CEF penumbra (see cyan contours in Fig. 3.5a). In particular, those regions contain pixels, located exactly at the umbral/penumbral boundary (see yellow markers in Fig. 3.5b), where $B>7 \mathrm{kG}$ according with the inversions. Such field strengths, apart from being extremely high compared with typical penumbral field strengths, are also stronger than the umbral field itself (the umbral field at $\log (\tau)=0$ has a local maximum close 


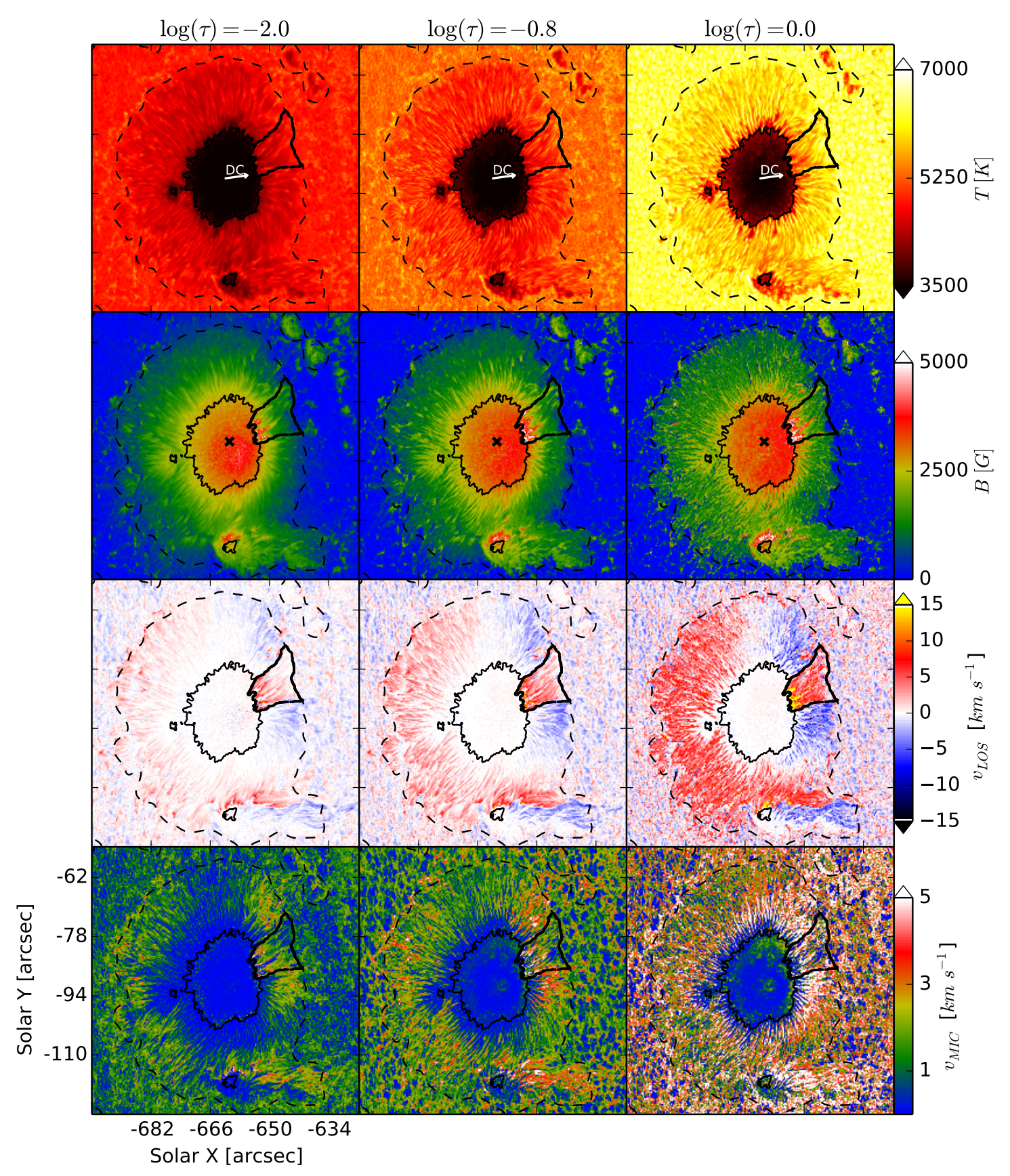

Figure 3.3: SPINOR 2D inverted parameters at three photospheric layers. From left to right: $\log (\tau)=-2.0,-0.8$ and 0 . From top to bottom: temperature $T(\mathrm{~K})$; magnetic field intensity $B(\mathrm{G})$; line-of-sight velocity $v_{L O S}\left(\mathrm{~km} \mathrm{~s}^{-1}\right)$; and the micro-turbulence velocity $v_{M I C}\left(\mathrm{~km} \mathrm{~s}^{-1}\right)$. In all maps, the black thick contour encloses a penumbral region where a counter EF is observed. The white arrows on the temperature maps point towards the disk center. Black crosses on the $B$ maps indicate the location of a local maximum of the umbral field strength at $\log (\tau)=0$. Also, the color-bar scale is sometimes saturated. 
3 Normal and counter Evershed flows in the photospheric penumbra of a sunspot: SPINOR 2D inversions of Hinode-SOT/ SP observations

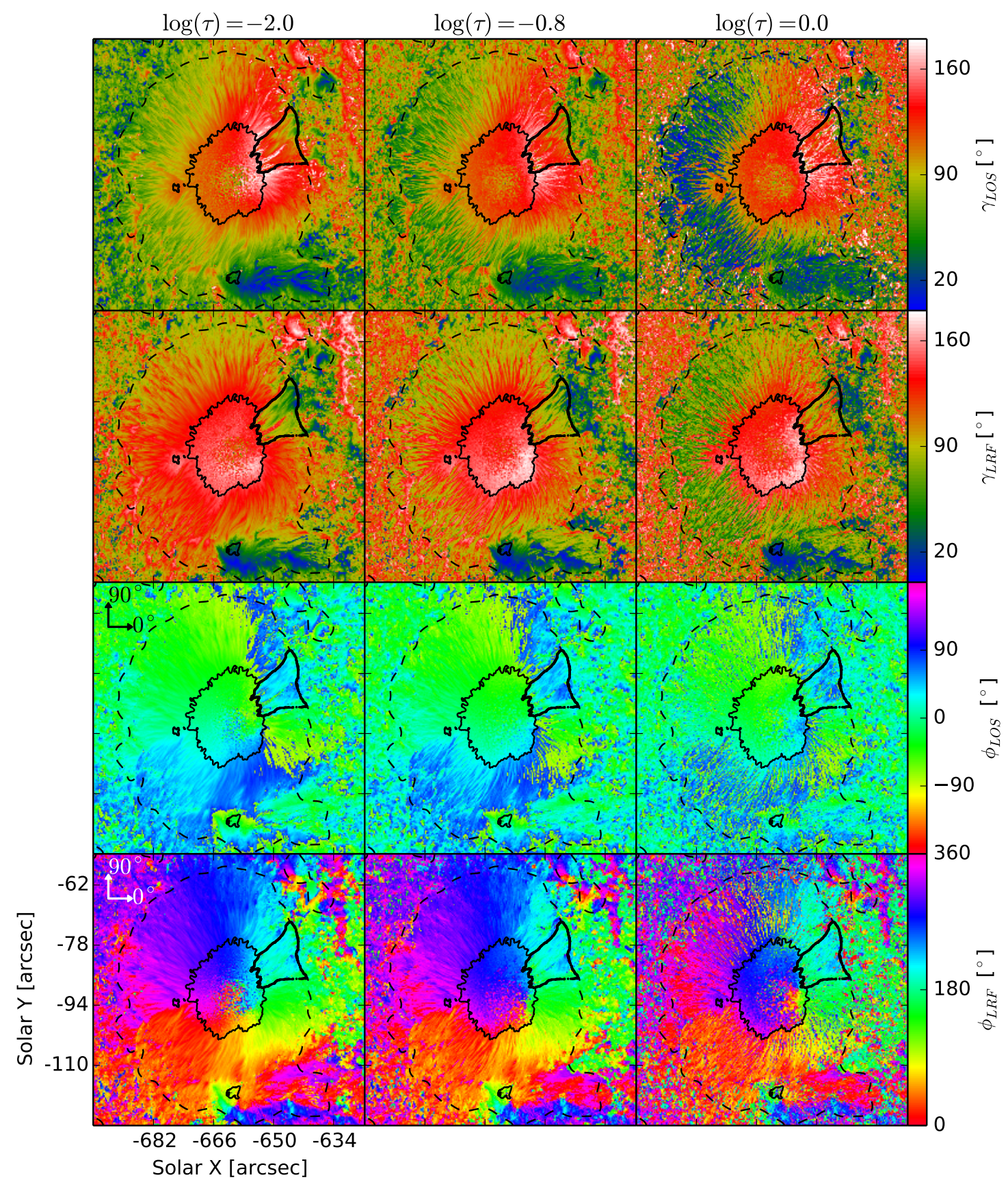

Figure 3.4: Field inclination and field azimuth from SPINOR 2D inversions at three photospheric layers. From left to right: $\log (\tau)=-2.0,-0.8$ and 0 . From top to bottom: the field inclination angle in the line-of-sight direction $\gamma_{L O S}\left({ }^{\circ}\right)$; field inclination in the local-reference-frame $\gamma_{L R F}\left({ }^{\circ}\right)$ after the disambiguation of the field azimuthal angle; the ambiguous field azimuthal angle in the line-of-sight direction $\phi_{L O S}\left({ }^{\circ}\right)$; and the disambiguated field azimuthal angle in the local-reference-frame $\phi_{L R F}\left({ }^{\circ}\right)$. The contours are the same as in Figure 3.3. 


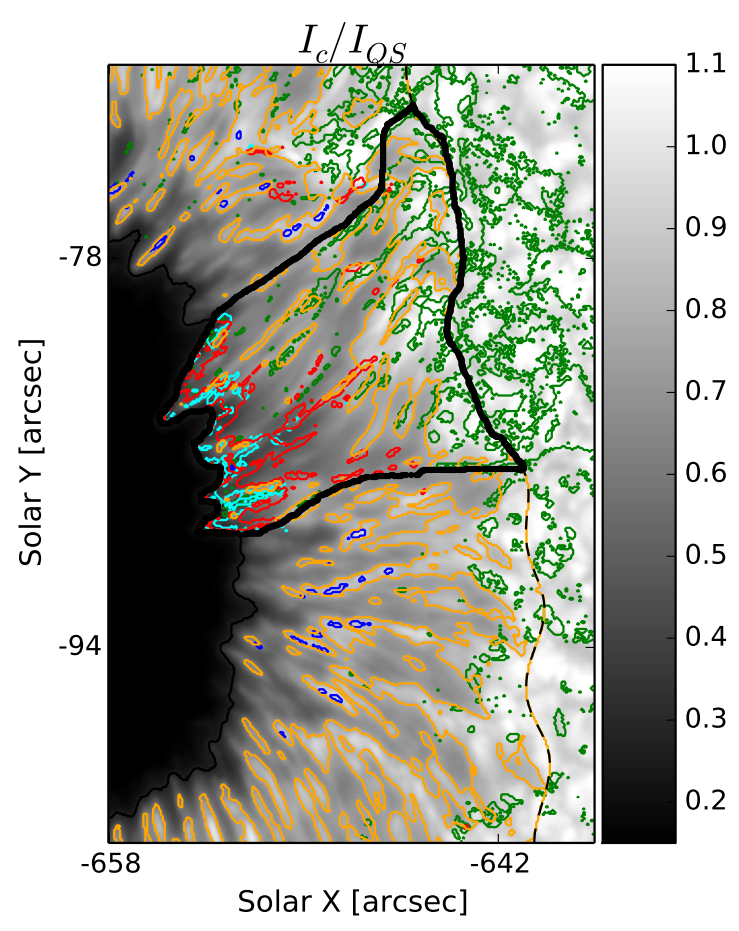

(a)

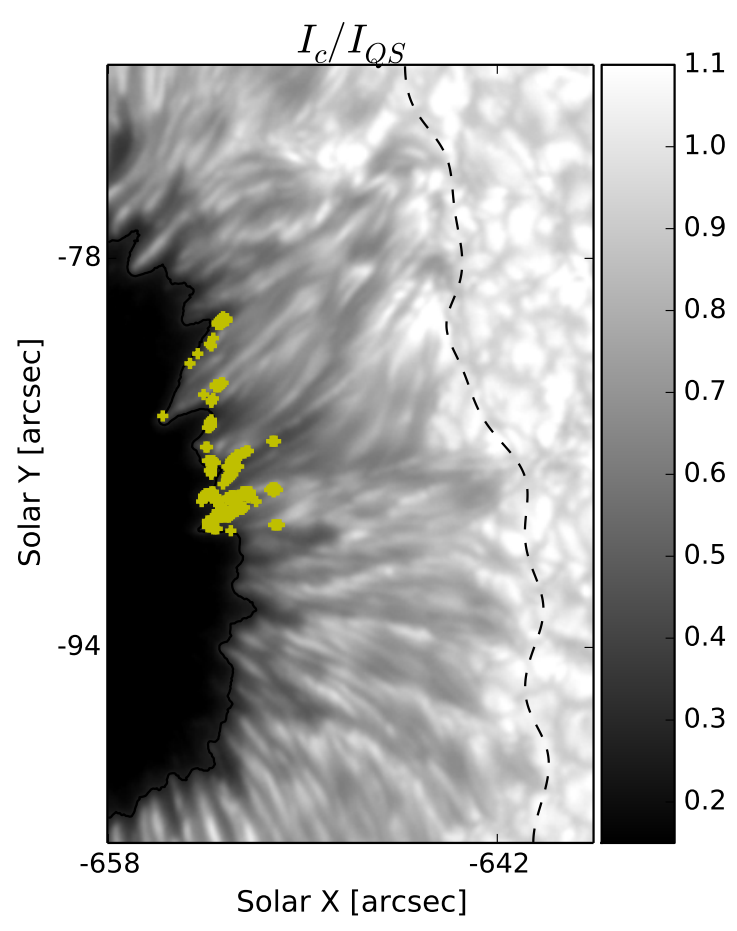

(b)

Figure 3.5: Continuum intensity maps of the center-side penumbra. (a) The black thick contour encloses the CEF region. The colored contour lines enclose the regions with lineof-sight velocities exceeding $9 \mathrm{~km} \mathrm{~s}^{-1}$ (red), regions with $v_{L O S}<-9 \mathrm{~km} \mathrm{~s}^{-1}$ (blue), regions where $B>5 \mathrm{kG}$ (cyan) and regions where $T>6000 \mathrm{~K}$ (orange) within the penumbra. The green contours enclose regions where $\gamma_{L R F}<90^{\circ}$ and the amplitude of Stokes $V$ is at least $10 \sigma$. All contouring was made at $\log (\tau)=0$. (b) Yellow marks indicate the location of pixels where the SPINOR 2D inversions return $B \geq 7 \mathrm{kG}$ at $\log (\tau)=0$.

to the umbra's geometric center as indicated by black crosses in the magnetic field maps of Fig. 3.3, where $B \sim 3.9 \mathrm{kG}$; although some pixels close to the center-side umbral boundary reach values up to $B \sim 4.2 \mathrm{kG}$ ).

Figure 3.4 shows the field inclination $\gamma$ and azimuth $\phi$ in both the LOS frame and LRF. The transformation of the magnetic field vector to the LRF suggests that the CEF region has different magnetic properties than the rest of the penumbra given that, at all three heights, the magnetic field lies more horizontally within the enclosed penumbral sector harboring the CEF, even changing polarity in the outer penumbra at $\log (\tau)=-0.8$ and -2 . The maps of $\gamma_{L R F}$ also show a number of patches of opposite polarity to that of the sunspot's umbra, in the center-side penumbra at all three optical depth levels. They are found mainly at the outer penumbral boundary in both the center-side NEF and the CEF regions; although there are many more opposite polarity patches concentrated closer to the outer penumbra in the CEF region. We can also see this in Figure 3.5a, where green contours have been placed on the continuum intensity map for the center-side penumbra enclosing regions where $\gamma_{L R F}<90^{\circ}$ at $\log (\tau)=0$ and the amplitude of Stokes $V$ is at least $10 \sigma$.

The overall variation of $v_{L O S}, T$, and $B$ with height in the CEF part of the penumbra is 


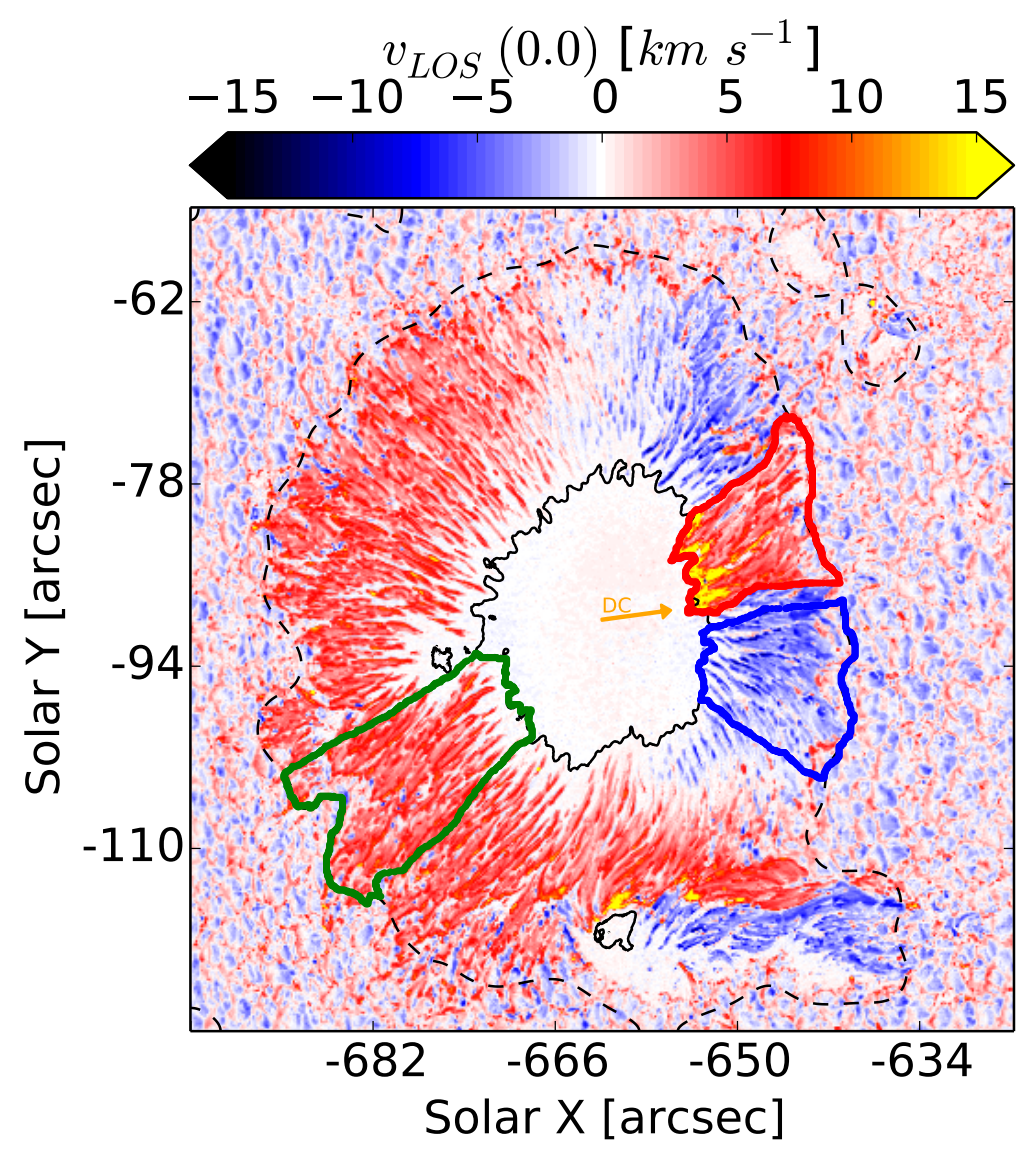

Figure 3.6: Three regions of interest are highlighted on the $v_{L O S}$ map at $\log (\tau)=0$ : region with CEF (red), center-side region with NEF (blue), and limb-side region with NEF (green).

similar to that of the NEF part; they all increase with depth (see Fig. 3.3). Nevertheless, in the anomalous region, $v_{L O S}$ and $B$ are observed to reach more extreme values. In particular, in the inner penumbra of the CEF region, $v_{L O S}>20 \mathrm{~km} \mathrm{~s}^{-1}$ and $B>5 \mathrm{kG}$ are observed at $\log (\tau)=0$. Such high values of $v_{L O S}$ and $B$ are atypical in the penumbrae of sunspots, with the closest values to these being those reported by van Noort et al. (2013). Furthermore, field strengths $B>7 \mathrm{kG}$ are unusual even for a dark umbra (see Livingston and Harvey 2006). We do not include the pixels where $B>7 \mathrm{kG}$ in the following analysis. Instead, we plan to conduct a more detailed study on the reality of unusually strong fields found in sunspot penumbrae. This is beyond the scope of this paper and will be the topic of future work ${ }^{2}$.

\subsubsection{Filament selection}

In order to compare the differences and/or similarities between the fine-scale structures related to the $\mathrm{CEF}$ and the NEF, we investigate now the generic properties of the filaments that populate the three penumbral regions identified in Figure 3.6. For this, we manually

\footnotetext{
${ }^{2}$ Part of this work is presented in Section 5 .
} 


\begin{tabular}{c|ccccc}
\hline & $l_{\text {mean }}(\operatorname{arcsec})$ & $l_{\text {std }}(\operatorname{arcsec})$ & $l_{\text {median }}(\operatorname{arcsec})$ & $w(\operatorname{arcsec})$ & $l / w$ \\
\hline NEF (center-side) & 5.2 & 1.4 & 5.0 & 0.56 & 9.29 \\
NEF (limb-side) & 4.9 & 1.3 & 4.5 & 0.80 & 6.13 \\
CEF & 9.4 & 2.7 & 11.0 & 0.96 & 9.79 \\
\hline
\end{tabular}

Table 3.1: Geometrical properties of the filaments: mean length $\left(l_{\text {mean }}\right)$, standard deviation $\left(l_{\text {std }}\right)$ and median values $\left(l_{\text {median }}\right)$ for each filament set, average filament width $(w)$, and the corresponding length-to-width ratio $(l / w)$.

selected six filaments from each of these penumbral regions.

The individual filaments were selected based on selection criteria introduced by Tiwari et al. (2013), but adapted to a sunspot located off the disk center $\left(\theta \approx 47^{\circ}\right)$. In our selection criteria, we use the temperature, the field inclination angle, and the line-of-sight velocity, at $\log (\tau)=0$, as follows: (1) The heads of the filaments are identified by relatively warm upflows and nearly vertical magnetic fields; (2) the bodies of the filaments are characterized by more horizontal fields and by the signature of the Evershed flow in $v_{L O S}$ due to the large heliocentric angle of the sunspot; and (3) the tails of the filaments are localized in regions of concentrated downflows and of nearly vertical fields of opposite polarity to the umbra.

The selection procedure was applied manually by placing points along the central axis of the filament. A line connecting these points was then computed using a bi-cubic spline interpolation. The path defined in this way defines the possibly curved axis, or spine of the filament and was used in the de-stretching and length normalization of the filament.

To perform the de-stretching and length normalization, 200 equidistant points were placed along the axis of each filament, after which ten points on a line perpendicular to the tangent were placed at intervals of one pixel $\left(0.08^{\prime \prime}\right)$ on each side of the path. A cubic interpolation of the inverted parameters in these points results in a de-stretched, de-rotated and length-normalized filament, as shown in Figure 3.7 for two individual filaments, one from the center-side NEF and one from the CEF penumbral regions.

With the method described above, a total of 18 penumbral filaments were selected and de-stretched, 6 inside each of the three penumbral regions highlighted in Figure 3.6. Our original aim was to select equal numbers of filaments located in the inner, middle, and outer parts of the penumbra within each highlighted region. Due to geometrical constraints introduced when using $\gamma$ as a selection parameter, it turned out to be most reliable to identify filaments in the inner penumbra in the limb-side NEF region. Likewise, all selected filaments in the center-side NEF region lie in the outer penumbra. In contrast, the filaments in the CEF region turned out to be much longer; most of them extend over the whole penumbral width, that is, they originate close to the boundary between penumbra and quiet Sun (i.e., this is where the upflow in these filaments is located) and end at the edge of the umbra. Such a positional difference between the filaments from each group should, however, not prevent us from performing a qualitative comparison between the filaments from the three regions, since, according to Tiwari et al. (2013), all filaments have essentially the same structure independently of their location within the penumbra, with the biggest differences happening in their surroundings.

Table 3.1 shows the mean, the standard deviation, and the median values of the length 
3 Normal and counter Evershed flows in the photospheric penumbra of a sunspot: SPINOR 2D inversions of Hinode-SOT/ SP observations

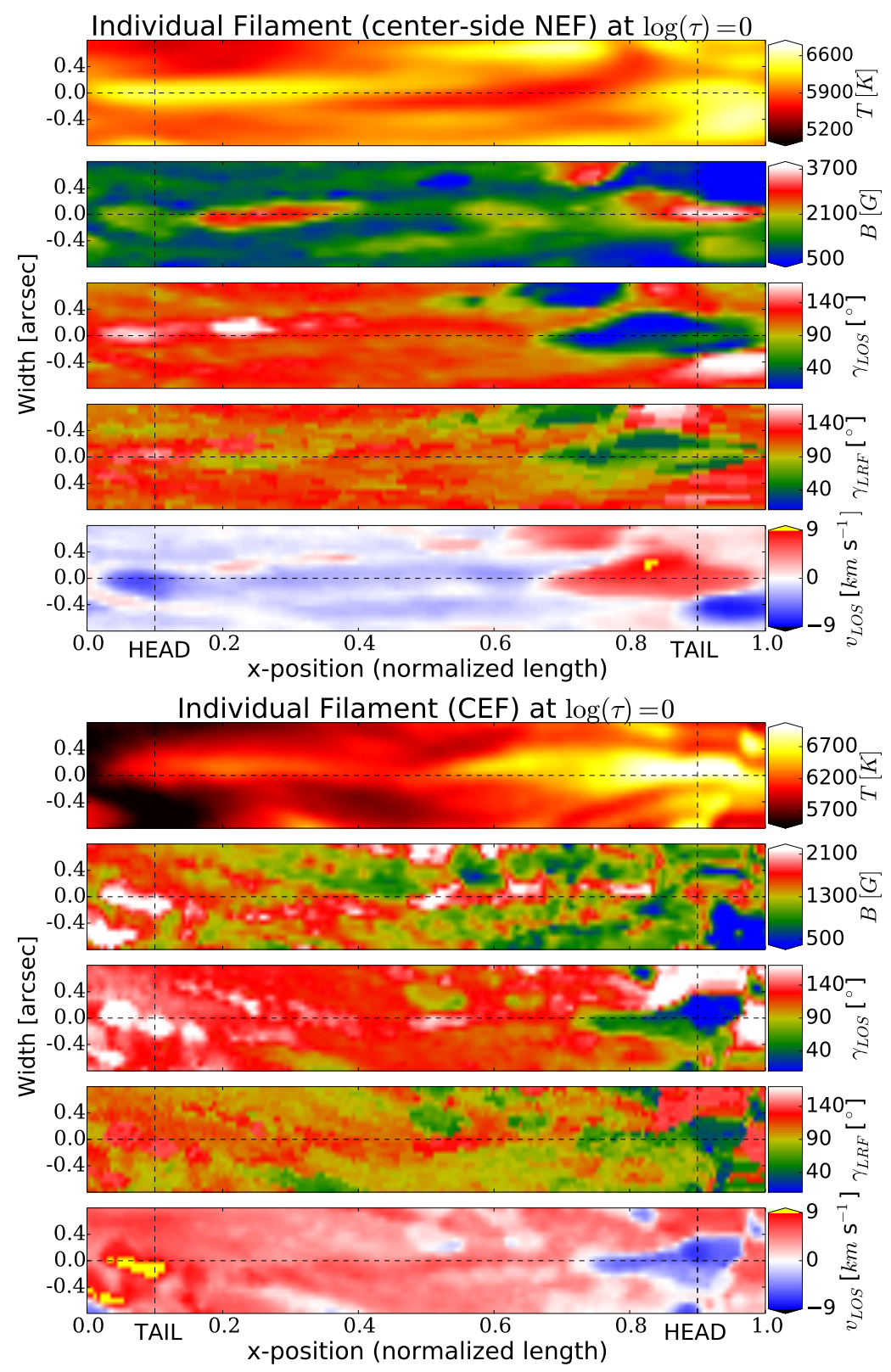

Figure 3.7: Individual filaments carrying a NEF (top) and a CEF (bottom) in their destretched and scaled form as seen at $\log (\tau)=0$. Subplots show, from top to bottom: the temperature $T$, magnetic field strength $B$, field inclination in the line-of-sight $\gamma_{L O S}$ and in the local reference frame $\gamma_{L R F}$, and the line-of-sight velocity $v_{L O S}$. The vertical dashed lines indicate transversal cuts close to the filament's endpoints: inner or closer to the umbra (left) and outer or closer to quiet sun (right). 'Head' refers to the footpoint harboring an upflow, 'tail' to the footpoint showing a downflow. The horizontal dashed lines indicate the de-stretched and length-normalized axis of the filament. The color-bars for a given parameter do not always have the same range for both maps of a given physical parameter and some of them have been saturated to highlight some patterns. 


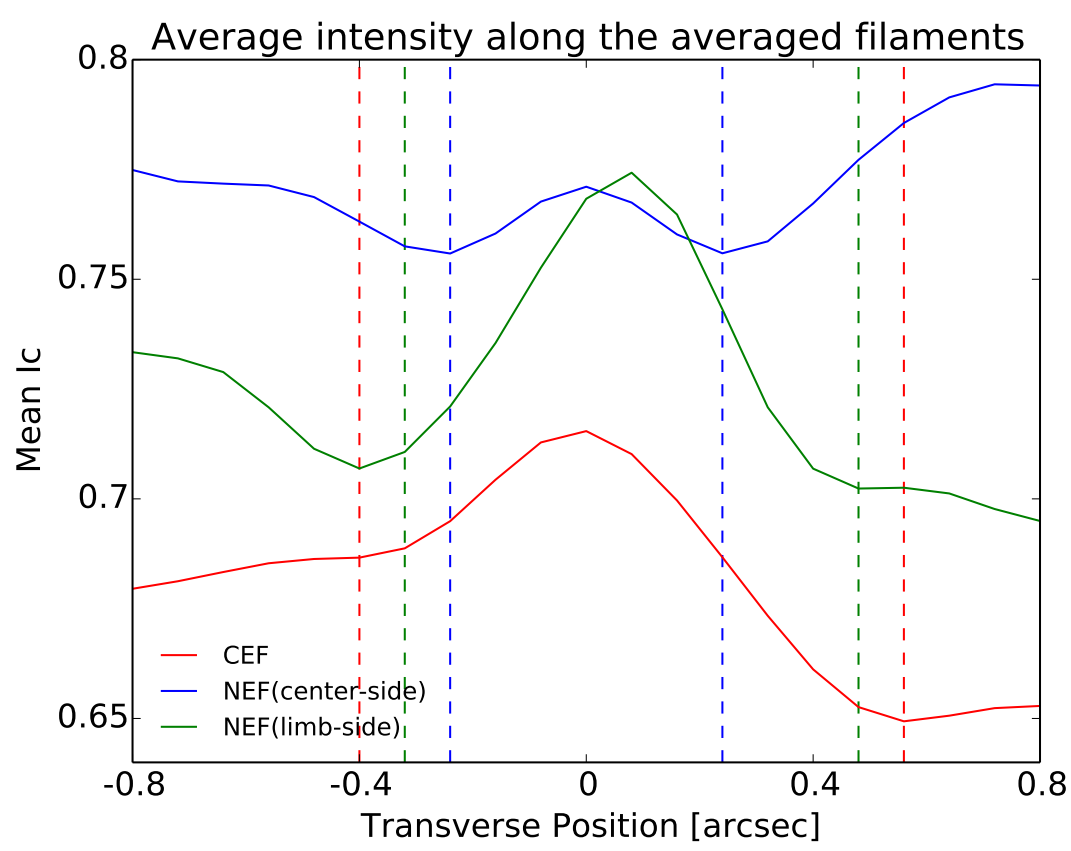

Figure 3.8: Transversal continuum intensity profiles of the average filaments. The plotted $I_{C}$ is averaged along the axes of the three averaged filaments: CEF (red line), center-side NEF (blue line) and limb-side NEF (green) regions.

distributions for each of the selected filament sets in the penumbra. Strikingly, the CEFcarrying filaments are nearly twice as long as those hosting the NEF.

Given the range of lengths of filaments from each selected region, they are rescaled prior to averaging. It is important to keep in mind that, while averaging, the variation in the length will cause some properties to be re-scaled along the filament. Nonetheless, the generic physical properties and structure, reflected by the average of such filaments, should be valid for filaments of all sizes.

Thus, after de-stretching and length normalizing all the selected filaments, we separately averaged each six-filament set in order to highlight their common characteristics, producing a "standard" filament for each of the three selected parts of the penumbra. This reduces the large variability in the appearance of the filaments caused by interactions with the environment as well as due to their intrinsic variability. Thus we finally analyzed only the above three standard or averaged filaments, one for each penumbral region marked in Figure 3.6.

The width of the filaments is, unlike their lengths, not re-scaled. The width values shown in Table 3.1 were computed by averaging the continuum intensity along the axes of the three averaged filaments. As shown in Figure 3.8, they come out to be roughly $0^{\prime \prime} .6,0^{\prime \prime} .8$ and 1 " for the center-side NEF, limb-side NEF and CEF regions, respectively. Thus, from the average of the measured lengths of the individual filaments, we arrive at a length-to-width ratio of approximately 9, 6 and 10 for the center-side NEF, limb-side $\mathrm{NEF}$, and CEF regions, respectively. 
3 Normal and counter Evershed flows in the photospheric penumbra of a sunspot: SPINOR 2D inversions of Hinode-SOT/ SP observations
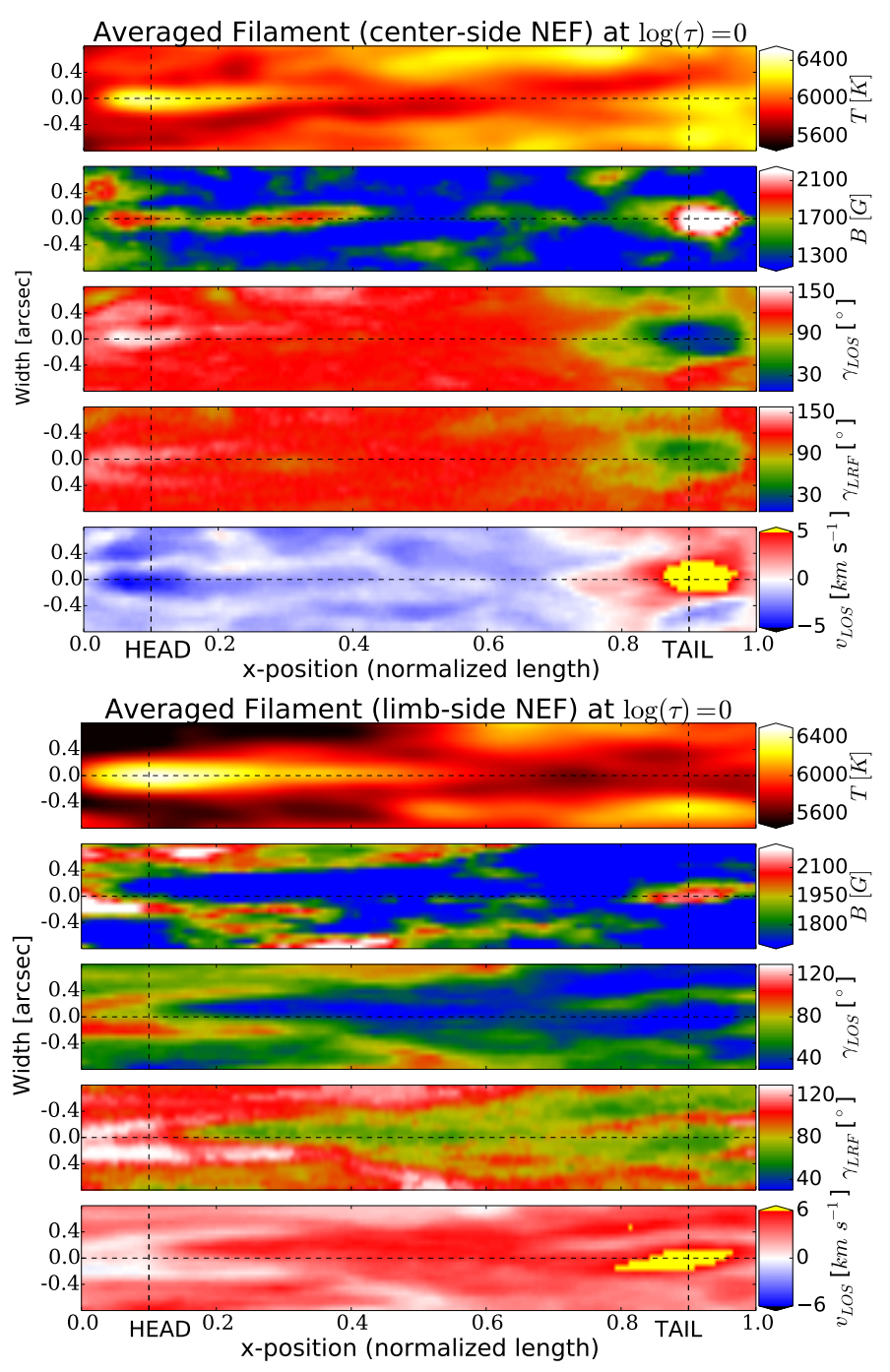

Averaged Filament (CEF) at $\log (\tau)=0$

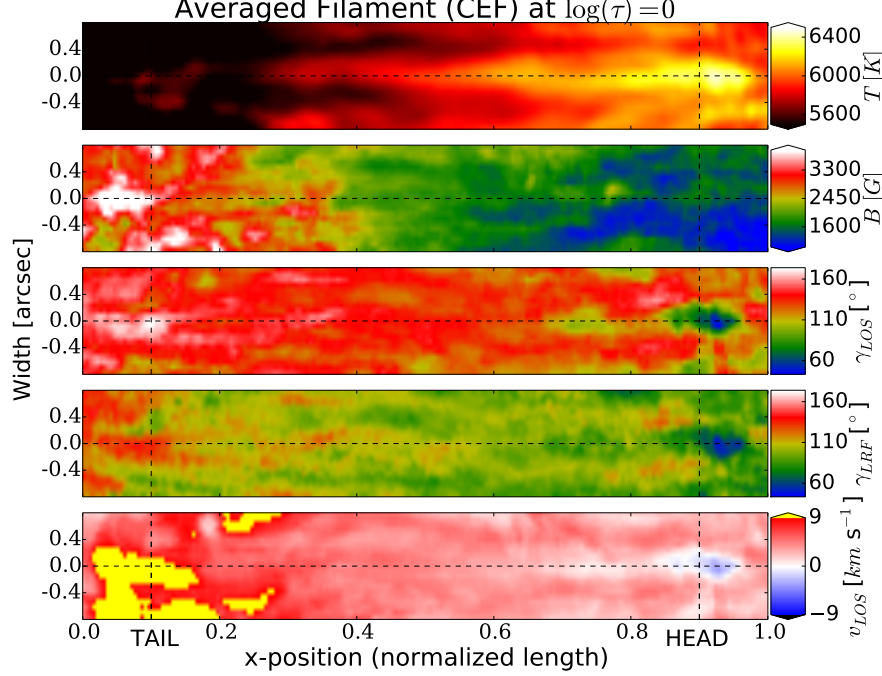

Figure 3.9: Average filaments from the center-side NEF (upper set of panels), limb-side NEF (middle panels) and CEF (lower panels) region. Subplots, that is, the set of five panels depicting filaments of a particular penumbral region, are in the same format as plots in Figure 3.7. The head of CEF filament appears located on the right, while the NEF filaments are plotted with their heads on the left, that is, the filaments are plotted such that the part closer to the umbra is on the left for all three cases. 


\subsubsection{Qualitative picture of filaments}

Figure 3.9 depicts a selection of properties of each of the three averaged filaments, representing the selected penumbral region (center-side NEF, limb-side NEF and CEF), at $\log (\tau)=0$. We concentrate on $\log (\tau)=0$ since the important differences and similarities between the averaged filaments are best seen there.

By comparing plots in Figure 3.9 with similar images of the individual filaments (e.g., Fig. 3.7) we can see that, averaging six filaments in each of the three groups is sufficient to clearly highlight the common features of the filaments and to suppress the largest individual fluctuations.

\subsubsection{Center-side and limb-side NEF filaments}

Figure 3.9 shows that the average filament from the center-side NEF region starts with a strong peak in temperature $(\sim 6400 \mathrm{~K}$ in the head). The temperature gradually decreases along the body of the filament and increases again towards the tail, up to $\sim 6300 \mathrm{~K}$. The magnetic field strength also decreases from the head towards the body from almost 1.9 $\mathrm{kG}$ to $\sim 1.3 \mathrm{kG}$, but then shows a local large strengthening at the very tail up to almost $2.5 \mathrm{kG}$.

The LOS inclination angle suggests that the head of the center-side filament has a downward pointing field, a more horizontal but slightly downward pointing field along the body (i.e., with the same polarity as the umbra), while the tail displays an opposite field polarity to that observed at the head, that is, upward-pointing field lines. This field configuration is maintained after transforming $\gamma$ to the LRF, that is, the transformation gives $\gamma_{L R F} \sim 140^{\circ}$ in the head, $\sim 110^{\circ}$ along the body and $\sim 60^{\circ}$ in the tail.

The LOS velocity shows strong blueshifts $\left(v_{L O S} \sim-5 \mathrm{~km} \mathrm{~s}^{-1}\right)$ concentrated in the head of the center-side filament, and weaker blueshifts $\left(v_{L O S} \sim-2 \mathrm{~km} \mathrm{~s}^{-1}\right)$ that continue in a thin band along the central axis of the filament. In contrast, large redshifts $\left(v_{L O S}>5\right.$ $\mathrm{km} \mathrm{s}^{-1}$ ) appear concentrated in the tail. This can also be clearly seen in Figure 3.10, where the thermal, the magnetic, and the velocity profiles along the central axes of the three average filaments are plotted (the corresponding cuts are indicated by horizontal dashed lines in Fig. 3.9).

From Figures 3.9 and 3.10 one can find various similarities between the center-side and the limb-side averaged NEF filaments: the temperature at the head of the limb-side NEF filament is roughly the same as in the center-side NEF case, and it drops from the head towards the tail, while the magnetic field suffers a notable strengthening near the tail. As in the center-side NEF filament, the limb-side NEF filament shows a slight temperature increase at the tail with respect to the body, assuming temperatures of up to $\sim 5800 \mathrm{~K}$. The field strength reaches values up to $\sim 2.2 \mathrm{kG}$ in the tail.

Figure 3.9 also shows that the flow is mainly concentrated along the body of the limbside NEF filament, reaching velocities $v_{L O S}>6 \mathrm{~km} \mathrm{~s}^{-1}$ in the tail. However, in this case we do not observe flows in the heads, likely because the orientation of the magnetic field is almost perpendicular to the LOS direction in that region. Assuming that the flow and the field have the same direction, no flows could then be observed along the LOS.

In addition, unlike in the center-side NEF case, the LOS inclination does not show the opposite field polarity between the head and the tail of the limb-side filament. Instead, we see in the head $\gamma_{L O S} \sim 80^{\circ}, \gamma_{L O S} \sim 40^{\circ}-50^{\circ}$ along the body, and $\gamma_{L O S} \sim 20^{\circ}$ in the 
3 Normal and counter Evershed flows in the photospheric penumbra of a sunspot: SPINOR 2D inversions of Hinode-SOT/ SP observations

tail. Nonetheless, once the field inclination is transformed into the LRF, the opposite field polarities between the filament endpoints and a roughly horizontal field along the body are unveiled: $\gamma_{L R F} \sim 120^{\circ}$ in the head (slightly downward pointing field), $\gamma_{L R F} \sim 80^{\circ}$ along the body (almost horizontal field) and $\gamma_{L R F} \sim 60^{\circ}$ in the tail (slightly upward pointing field).

The slightly different curvatures of the field along the center-side and limb-side NEF filaments, as seen in the LRF (see sketch in Figure 3.12), cannot be attributed to their different radial locations within the penumbra (outer and inner filaments, respectively) since, according to the findings of Tiwari et al. (2013), the field inclination is remarkably independent of the location of the filament, displaying only modest differences between filaments at different radial distances.

There are multiple possibilities for the different curvatures of the center-side and limb-side NEF filaments. One possibility is that we see somewhat different heights in the center-side and limb-side penumbra, as the ray passes through different atmospheric structures so that a given optical depth is reached at different heights in the two geometries. There are also biases introduced by the fact that Stokes $V$ is typically significantly stronger than Stokes $Q$ or $U$, so that a magnetic field directed (anti-) parallel to the LOS gives a larger contribution than one that is directed perpendicular to the LOS. If there is a mixture of unresolved fields with different strengths and inclinations (or if SPINOR does not remove all the stray light), then this introduces a difference in the field strength (compare $B$ in the the heads and tails of the two NEF filaments in Figs. 3.9 and 3.10) and also in the inclination. Finally, the penumbra may be intrinsically asymmetric, with the body of the limb-side filaments formed by almost horizontal fields that are pointing inwards and slightly upwards, and the body of the center-side NEF filaments formed by also inward directed almost horizontal fields but pointing slightly downwards.

As expected, there are some remarkable differences seen in the surroundings of the averaged filaments from the center-side and limb-side NEF regions since they are the result of averaging groups of outer and inner filaments, respectively, and the physical parameters partly change quite considerably from the inner to the outer penumbra. Such differences are consistent with the results of Tiwari et al. (2013) for the surrounding environment of filaments located in the inner and outer penumbra. Even though the temperature in the surroundings of the filaments increases gradually with radial distance in both cases (i.e., when moving from umbra to the quiet Sun), the environment of the filament from the center-side NEF penumbra (outer filaments) is significantly hotter than in the limb-side NEF case (inner filaments). As argued by Tiwari et al. (2013), the differences in the properties of the surroundings are due to the variation of the spines with radial distances. Such differences appear to have an important effect on the downstream temperature structure of the filaments (tail of filament in center-side NEF region is hotter than the tail in the limbside case). This can be clearly seen in both Figures 3.10 and 3.11. In the latter, various physical parameters are plotted along two transversal cuts near the head (solid lines) and the tail (dashed lines), indicated by vertical dashed lines in Figure 3.9.

Figure 3.11 also shows that the magnetic field surrounding the head of the limb-side NEF filament (inner filaments) is significantly stronger than inside the head of the filament. In contrast, the magnetic field strength surrounding the head of the center-side NEF filament (outer filaments) is slightly weaker than the field strength inside the head. However, as clearly revealed by Figures 3.10 and 3.11, the field strength in the filament 

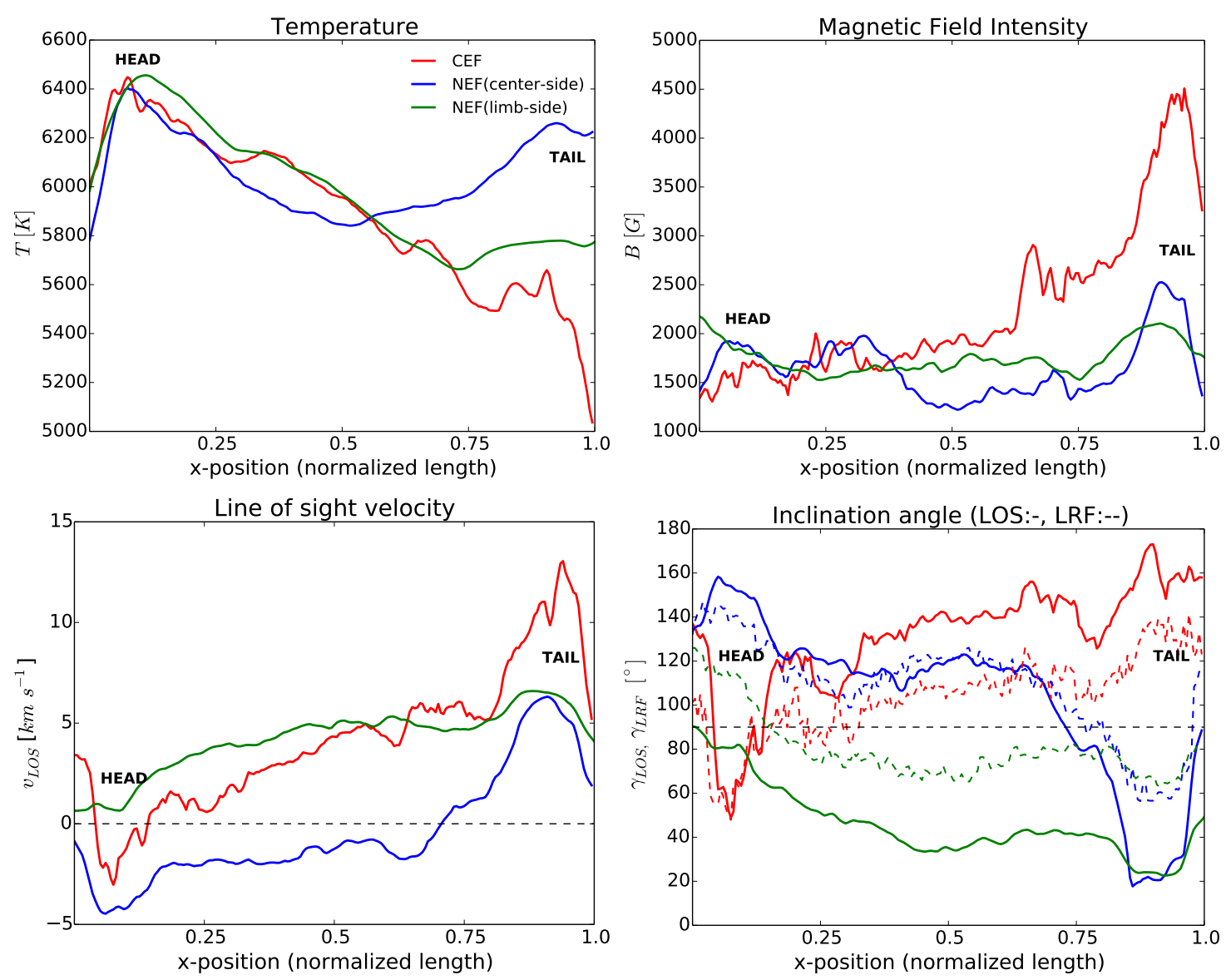

Figure 3.10: Thermal, magnetic and velocity profiles along the central axes of average filaments at $\log (\tau)=0$. Clockwise: temperature $T$, magnetic field strength $B$, field inclination in the line-of sight $\gamma_{L O S}$ (solid lines) and in the local reference frame $\gamma_{L R F}$ (dashed lines), and line-of-sight velocity $v_{L O S}$. The profiles correspond to the longitudinal cuts denoted by horizontal dashed lines in Figure 3.9: center-side NEF (blue), limb-side NEF (green) and CEF (red). Here, the "natural" x-position of the average filament from the CEF case has been reversed and we refer to its outermost footpoint (closest to the quiet Sun) as its 'head' and to the innermost one (closest to the umbra) as its 'tail'.

itself seems to be very similar for both, the center-side and limb-side NEF regions, independently of their different radial locations. Moreover, the field strength is $\geq 1 \mathrm{kG}$ everywhere inside both NEF filaments, and slightly larger than $2 \mathrm{kG}$ in their tails.

\subsubsection{CEF filaments}

Figure 3.9 also shows that the endpoints of the averaged filament from the CEF region are exchanged compared to the NEF case: the filament endpoint closer to the umbra displays properties similar to the tail of the NEF filaments, but being even more extreme, with a local large strengthening of the magnetic field $(\sim 4.5 \mathrm{kG})$ and large redshifts that correspond to supersonic line-of-sight velocities $\left(v_{L O S}>9 \mathrm{~km} \mathrm{~s}^{-1}\right)$; while the outer endpoint behaves as a head, being much hotter than its surroundings and harboring concentrated blueshifts. This clear spatial anti-correlation strongly suggests that the filaments in the 
3 Normal and counter Evershed flows in the photospheric penumbra of a sunspot: SPINOR 2D inversions of Hinode-SOT/ SP observations
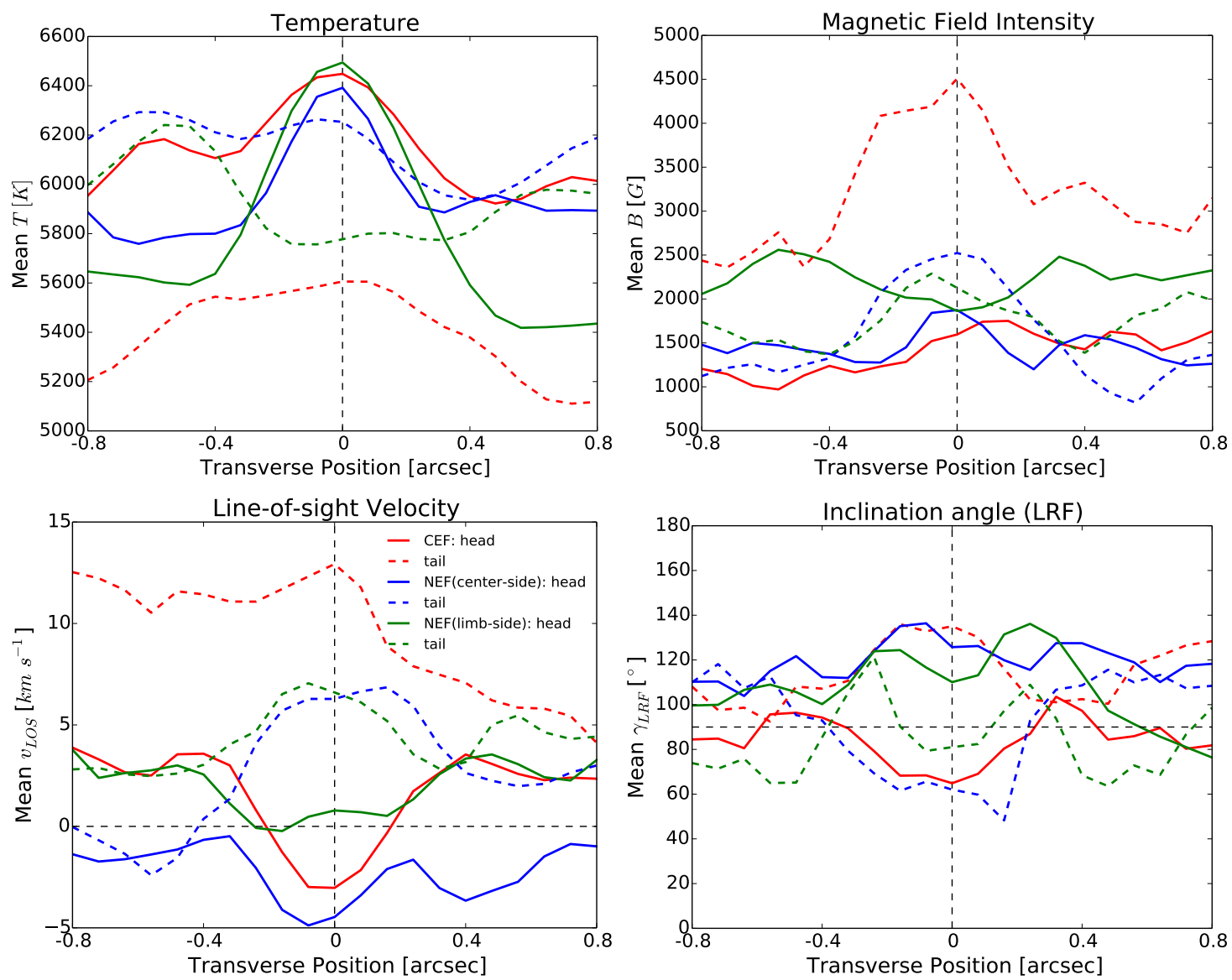

Figure 3.11: Variation of $T, B, v_{L O S}$ and $\gamma_{L R F}$ along the transversal cuts marked by vertical dashed lines in Figure 3.9 at $\log (\tau)=0$ : heads (solid lines) and tails (dashed lines). The color designation is the same as in Figure 3.10. Black dashed vertical lines represent the central axis of the averaged filaments.

CEF region are "reversed filaments", with their heads located at their outermost endpoints and their tails at their umbral directed endpoints.

According to Figure 3.9, the head of the averaged filament from the CEF region is followed by a thin band along the center of the filament where relatively low and positive values of $v_{L O S}$ are seen (in this case, the head lies close to the outer boundary of the spot and the filament extends towards the umbra). The LOS velocity gradually increases from head to tail along the central axis of the filament, from $\sim 1$ to $\sim 5 \mathrm{~km} \mathrm{~s}^{-1}$, which represents an inflow towards the umbra (counter Evershed flow) roughly along horizontal magnetic field lines $\left(\gamma_{L R F} \sim 90-110^{\circ}\right)$.

We interpret the observation of low $v_{L O S}$ values just after the head of the filament to be a result of the viewing geometry. In fact, the LOS velocity shows stronger redshifts around the head and in the lateral edges immediately after the head than inside the body of the filament (this is clearly visible mainly between 0.8 and 0.9 of the normalized x-position of Fig. 3.9). Those lateral redshifts are likely produced by lateral downflows occurring at the edges of the filament where the field is pointing downwards $\left(\gamma_{L R F} \sim 140^{\circ}\right)$. The dominance of the lateral downflows on the velocity transversal profile of that region can 


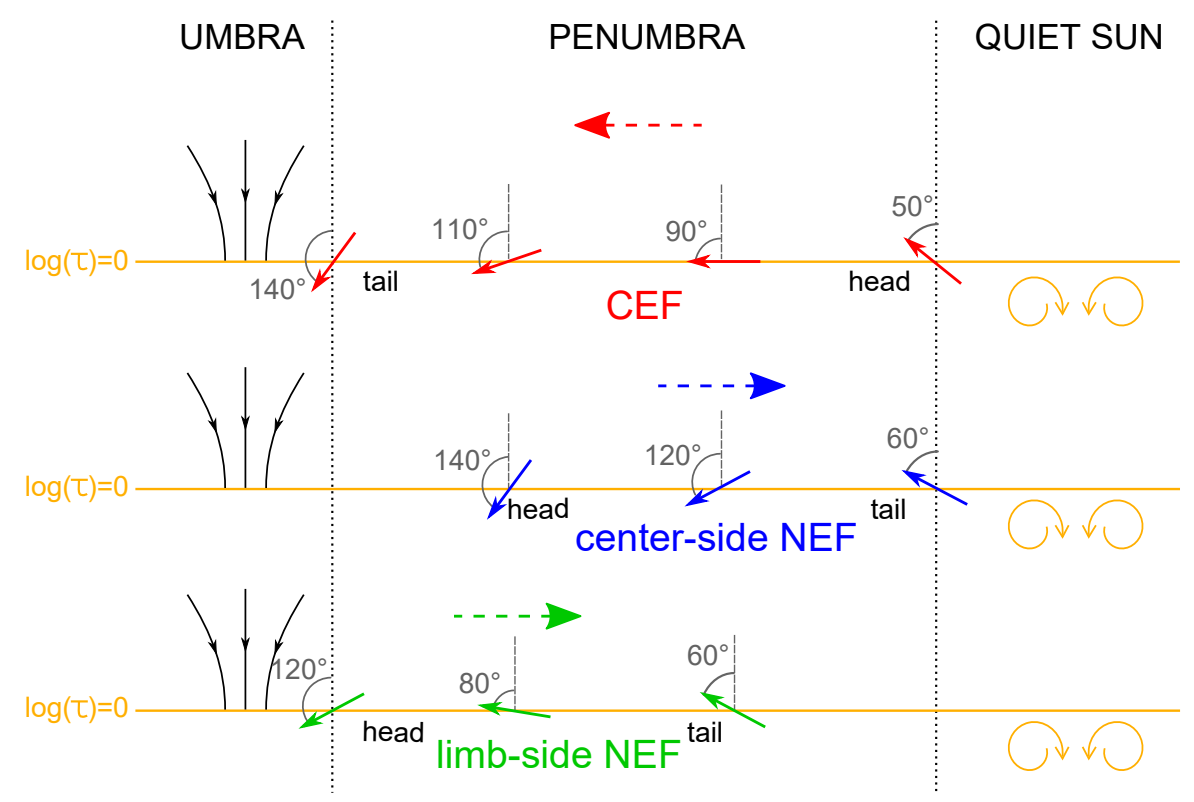

Figure 3.12: Possible magnetic field configuration of the three average filaments from different sectors of the penumbra, according to $\gamma_{L R F}$ at $\log (\tau)=0$. Solid arrows represent the magnetic field inclination in the CEF average filament (red), center-side NEF (blue) and the limb-side NEF (green) average filaments. The dashed horizontal arrows indicate the radial flow direction.

also be explained by the viewing geometry: there, the field is more aligned to the LOS direction $\left(\gamma_{L O S} \sim 150^{\circ}\right)$ than in those regions of weak redshifts along the central axis of the filament (where $\gamma_{L O S} \sim 110^{\circ}$ ).

According to $\gamma_{L R F}$, the CEF would flow almost horizontally right after the head $\left(\gamma_{L R F} \sim\right.$ $90^{\circ}$ ), assuming a coupled bending of the field and the flow immediately after the head. The tail in this case shows strong, supersonic LOS flows that we interpret as downflows, which coincide spatially with a large enhancement of the magnetic field strength and with downward pointing fields $\left(\gamma_{L R F} \sim 140^{\circ}\right)$.

\subsubsection{Comparison between NEF and CEF filaments}

Plots in Figure 3.10 disclose a qualitatively similar behavior of the temperature, field strength, and line-of-sight velocity along the central axes of the three averaged filaments, from the "heads" towards the "tails".

In all three cases, there is a systematic decrease of the temperature from the head towards the body of the filaments. Despite a local temperature increase in the tails, the heads are always hotter than the tails. This temperature difference is $\Delta T \sim 800,600$, and $100 \mathrm{~K}$ for the CEF, limb-side NEF, and center-side NEF case, respectively . Also, in all three cases, the tails display large to very large enhancements in the magnetic field strength, which, compared to the heads, give $\Delta B \sim 3000,600$, and $300 \mathrm{G}$ for the CEF, center-side NEF, and limb-side NEF case, respectively. In this case, because the head of the CEF-carrying filaments is lying at the outer penumbra, a larger $B$ value at its tail compared with the head is expected because $B$ is on average larger by $1-1.5 \mathrm{kG}$ near the 
3 Normal and counter Evershed flows in the photospheric penumbra of a sunspot: SPINOR 2D inversions of Hinode-SOT/ SP observations

umbra than at the outer penumbral boundary (Solanki 2003, Tiwari et al. 2015). This explains most, if not all of the difference in $\Delta B$ between CEF- and NEF-carrying filaments. Something also worth noticing is that the bodies of the filaments are not field-free gaps, but are actually magnetized with strengths above $1 \mathrm{kG}$ everywhere in the penumbra, in agreement with the findings of Tiwari et al. (2013).

In all three cases, there are concentrated flows in the tails, with $v_{L O S}>5 \mathrm{~km} \mathrm{~s}^{-1}$. In particular, the tail of the standard filament carrying the CEF displays supersonic velocities $\left(v_{L O S}>9 \mathrm{~km} \mathrm{~s}^{-1}\right)$. Such supersonic flows might be contributing to the enhancements in temperature seen at the tails, maybe due to the formation of shocks as suggested by Tiwari et al. (2013).

The LRF inclination angle profiles show opposite field polarities between heads and tails in all three average filaments. To help the reader visualize the magnetic configuration of the average filaments at $\log (\tau)=0$, in Figure 3.12 we have sketched the magnetic field inclination of the three average filaments, according to the information provided by $\gamma_{L R F}$ in the deepest visible layer: The head of the center-side and limb-side NEF filaments have both the same polarity as the umbra $\left(\gamma_{L R F} \sim 140^{\circ}\right.$ and $120^{\circ}$, respectively), while the CEF filament head has the opposite polarity $\left(\gamma_{L R F} \sim 50^{\circ}\right)$. The magnetic field displays roughly the same general magnetic polarity in both the CEF and center-side NEF parts of the penumbra when looking at both from the umbra outwards. The anti-correlation between their $\gamma_{L R F}$ profiles at the heads and the tails of NEF and CEF, seen in Figures 3.10 and 3.11 , is a result of plotting the filaments such that the heads (i.e., upflows) are together. The different radial position within the penumbra of the center-side and limb-side NEF filaments (outer and inner filaments, respectively), as well as the different radial extent of the CEF filaments, have been sketched in Figure 3.12.

From the plots in Figure 3.11, we can again find considerable similarities in the behavior of the two endpoints of the averaged filaments in different sectors of the penumbra, with the biggest differences being observed between their surroundings. The plasma surrounding the tail of the CEF averaged filament is substantially cooler than in the NEF cases. This is not surprising since most of the filaments in the CEF region are seen to penetrate the umbra (see Figure 3.3) and consequently the tails are located in a relatively cool environment (which is even cooler than the media surrounding the heads of the inner filaments in the limb-side NEF region).

In summary, we could say that the flows (both, the NEF and the CEF) all start as upflows in the bright and hot head of the filament containing them, where the field is more vertical. The material is then carried radially outwards/inwards along the NEF/CEF filament's axis. Along the way, the gas cools and finally sinks again at the tails, where the gas is cooler than in the heads and the field is stronger.

\subsection{Discussion}

In the center-side penumbra, one would normally expect to observe blue-shifts or plasma flows directed towards the observer due to the well-known photospheric Evershed effect (Evershed 1909), a radial and almost horizontal outward-directed flow of material. However, the observed redshifts in a sector of the center-side penumbra of the main spot of AR NOAA10930, caused by plasma motions pointing away from the observer, indicate either 


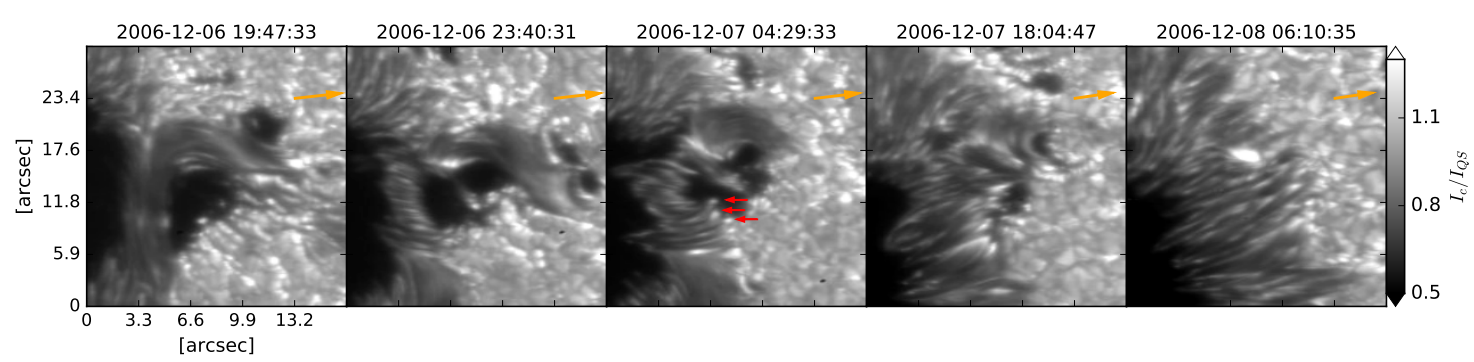

Figure 3.13: Hinode G-band images showing the evolution of the anomalous penumbra in AR 10930, at five selected stages. Yellow arrows point towards the disk center. The red arrows in the third frame show the location of penumbral bright grains.

inward-directed motions (towards the umbra) or downflows in the center-side penumbra. Under the assumption of flows being aligned with the magnetic field, such a red-shifted region in the center-side penumbra represents a counter-Evershed flow. We discard the possibility that these redshifts could be produced by a nearly vertical downflow of plasma only, due to the finding of an associated radial penumbral fine structure (i.e., filamentary fine structure) and further similarities with the normal Evershed outflow. Nonetheless, the observation of strong redshifts in regions with more vertical fields at the edges of the filaments carrying the CEF suggests the existence of lateral downflow channels.

The very large line shifts and line splittings observed in the anomalous penumbral region, and the extensive area that the CEF spans, make this sunspot a rather unique one. The previously reported observations of CEFs by, for example, Kleint and Sainz Dalda (2013), Louis et al. (2014), have been mainly restricted to penumbral filaments of sunspots leading to flares, or have been found in forming penumbrae (Schlichenmaier et al. 2012, Romano et al. 2014, Murabito et al. 2016).

Kleint and Sainz Dalda (2013) propose two possible models to explain the observation of CEFs in the photosphere. The first model is the umbral filament sheet model, in which the CEF would occur along a topological feature in the form of a sheet that magnetically connects spatially separated regions (e.g., the umbra with a network element well outside the spot), the CEF is then described as a siphon flow produced by the pressure difference between the umbral base of the sheet and the magnetically connected network element. The second model proposed by Kleint and Sainz Dalda (2013) is the massive umbral filament model, in which a thick flux tube with higher density than the penumbra carries the common chromospheric inverse EF, with the NEF hidden below the flux tube.

In our study, we have not analyzed the dynamics of the anomalous part of the penumbra in the higher layers of the atmosphere. We have concentrated mainly on the deepest visible layer $(\log (\tau)=0)$, where the sources of the CEF could be identified within the penumbra itself (at the outer border). On the one hand, the fact that we see a CEF at the deepest observable layers in an otherwise nearly normal photospheric penumbra, is not compatible with the massive umbral filament model of Kleint and Sainz Dalda (2013), which basically describes the CEF as a chromospheric flow. On the other hand, the fact that the CEF begins and ends within the penumbra itself, is conflicting with the umbral filament sheet model, which requires that the flow is driven from a network element. Also, we do not observe turbulent motions in the boundary between the CEF and the NEF (perhaps due to the spatial resolution), as would be expected in the umbral filament 
3 Normal and counter Evershed flows in the photospheric penumbra of a sunspot: SPINOR 2D inversions of Hinode-SOT/ SP observations

sheet model. Instead, we observe that the CEF is concentrated along penumbral channels, whose magnetic and thermal structures strongly suggest that they are "inverted normal filaments" with their heads/flow-sources located at the outer penumbral boundary and the tails/flow-sinks located in the inner penumbral boundary.

Our analysis allows for two interpretations of the driver of the CEF (and the NEF). One possible qualitative picture of the CEF (and of the NEF) emerging from our analysis is that of a siphon flow driven by a gas pressure gradient due to different magnetic field strengths at the two endpoints of the flux tubes forming the penumbral filaments in the anomalous region, in accordance with the model of Meyer and Schmidt (1968a) for explaining the driving forces of the NEF.

We found a field strength gradient of $\sim 3000 \mathrm{G}$ between the tail and the head of the averaged filament carrying the CEF $(\sim 300-600 \mathrm{G}$ in the averaged filaments carrying the NEF). This may contribute to the acceleration of the flows since an enhanced field in the tails implies a larger gas pressure in the head compared to the tail. However, to validate this siphon flow scenario, we need to know if a pressure gradient exists between the endpoints of the filaments at the same gravitational potential, that is, at constant geometrical height. This is unfortunately impossible to ascertain since the present observations provide physical information of constant optical depth layers only.

According to van Noort et al. (2013), the strongest fields in penumbrae are usually found at the ends of complex filaments, particularly those with multiple heads that merge to form a single tail. Those tails show a polarity opposite to that of the sunspot umbra and contain supersonic downflows ( $\geq 9 \mathrm{~km} \mathrm{~s}^{-1}$ ). van Noort et al. (2013) argue that the strong magnetic fields are probably the result of intensification of magnetic field by the collapse of magnetized flux concentrations (e.g. Parker 1978). In addition, the optical depth unity surface might be strongly depressed at the tails of the filaments, exposing stronger fields from a deeper geometrical height. In our study, we have only analyzed simple filaments (with a single head and a single tail) carrying the NEF and the CEF, respectively. The tails of these filaments also contain enhanced field strengths $(B \sim 2-2.5 \mathrm{kG}$ on average for the tails of NEF-carrying filaments and $B \sim 4.5 \mathrm{kG}$ on average for the tails of CEFcarrying filaments), and are co-located with fast downflows (within the subsonic regime for the NEF case, $v_{L O S} \simeq 7 \mathrm{~km} \mathrm{~s}^{-1}$ on average; and with supersonic speeds in the CEF case, $v_{L O S}>9 \mathrm{~km} \mathrm{~s}^{-1}$ ). The mechanisms considered by van Noort et al. (2013) are also a possible explanation for the relation between the large downflow velocities and the enhanced magnetic field strengths in our observations. Likewise, it is possible that the enhanced field strengths found in the tails of the filaments carrying both the CEF and the NEF, correspond to regions below the average geometrical height of the penumbra, as proposed by van Noort et al. (2013) and Tiwari et al. (2013). This could also explain the temperature enhancement found in the tails of the filaments, since we might be seeing deeper and hotter layers in the tails than in the other parts of the penumbra. The supersonic downflows observed in the tails of the filaments carrying the CEF might contribute to the increase in temperature due to the formation of shocks.

In particular, we do not discard the possibility that the very large magnetic field values returned by the SPINOR 2D inversions $(B>7 \mathrm{kG})$ are real and they are observed in the penumbra due to an unusually depressed optical depth surface formed as a consequence of very low densities in the downflowing part of the anomalous penumbra harboring the CEF. However, given that most of the pixels where SPINOR 2D returns $B>7 \mathrm{kG}$ are located 
at or close to the umbral/penumbral boundary of the CEF region (see yellow markers in Fig. 3.5b) and contain very complex Stokes profiles (e.g., they exhibit a large wavelength separation, large asymmetries and multi-lobed Stokes $V$ profiles), it is also possible that those profiles are produced by multiple (unresolved) atmospheric components with large differences in their Doppler velocity. One of the components could be associated with the umbral magnetic field in the sunspot (where the medium is nearly at rest) and the second one with the filamentary penumbra (strongly red-shifted component). Very large Doppler shifts as well as extremely strong magnetic fields could explain the large wavelength separation observed at these peculiar pixels in the umbral/penumbral boundary. However, in order to gain insight into the nature of these complex profiles it is necessary to perform a detailed analysis using, for example, some classical diagnostic methods and different inversion techniques considering different model atmospheres to see which one gives the most reliable results. This will be the topic of a future study ${ }^{3}$.

The other possible driver compatible with our results is the thermal gradient. The systematic temperature decrease from the heads to the tails observed in all three averaged filaments (limb-side NEF, center-side NEF and CEF) is compatible with the convective driver scenario, as proposed by Scharmer and Spruit (2006), Spruit and Scharmer (2006), but modified by the presence of a magnetic field since we observe field strengths $B>1$ $k G$ in the body of the filaments, similar to the findings of Tiwari et al. (2013) in filaments carrying a NEF. In this scenario, the upflowing hot gas reaches the solar surface due to the convective instability. There, the gas decelerates and builds up excess pressure. Due to the generally radial and horizontal magnetic field direction, the gas flows, to a large extent, radially along the body of the filaments. Along the way, the gas cools down and eventually sinks in the tails. This is in qualitative agreement with the results of the simulations of Rempel et al. (2009a). Independently of their opposite horizontal flow direction, in both cases (NEF and CEF), the relationship between the direction of flow and the temperature, with upflowing material being hotter than the downflowing material, could provide support for the presence of overturning convection along the penumbral filaments. Furthermore, since we might be seeing higher layers in the heads than in the tails, the temperature difference at an equal geometrical height between heads and tails should be considerably larger than the observed (Bruls et al. 1999). However, the relevant physical parameters need to be known on a geometrical scale to confirm this scenario.

The various opposite field polarity patches that are observed outside, but in the vicinity, of the anomalous part of the penumbra, might be related with the initiation of the $\mathrm{CEF}$, since this is apparently the only aspect that distinguishes (in the surroundings) the part of the penumbra with CEF from the other parts of the penumbra considered as normal. The lack of SP scans prevents us from observing the exact time at which the CEF is initiated in the anomalous part of the penumbra. However, Hinode observations of the G-band and narrow-band filtergrams show the presence of an adjacent pore with opposite magnetic polarity to that of the umbra of the main sunspot prior to the analyzed SP scan. This pore was located in the region where the opposite polarity patches are observed in the present SP scan (green contours in Fig. 3.5a), which might be remnants of the pore. A quick look into the temporal evolution of the pore in the G-band images (see Fig. 3.13) suggests that the pore develops a penumbra-like connection with the main sunspot (visi-

\footnotetext{
${ }^{3}$ Chapter 5.
} 
3 Normal and counter Evershed flows in the photospheric penumbra of a sunspot: SPINOR 2D inversions of Hinode-SOT/ SP observations

ble in the second frame). The CEF-carrying part of the penumbra developed out of this initial connection. The third frame shows that the penumbral filaments in the anomalous region grow while the area of the pore decreases. The location of the penumbral bright grains (red arrows in third frame) suggests that the filaments originate in the pore and extend outwards, towards the umbra of the main sunspot. Consequently, if a NEF is carried along those filaments (from the pore outwards), that would mean an inflow towards the umbra of the main sunspot in the AR, that is, a CEF. The fourth frame shows that the area of the pore continues decreasing while the penumbral filaments grow, and finally, in the last frame (which roughly corresponds to the time of the analyzed SP scan) the pore has disappeared and the penumbral filaments in the anomalous region seem to have been "adopted" by the main sunspot of the AR while carrying a CEF.

Jurčak et al. (2017) also reported the evolution of a penumbra at the boundary of a small pore, in which the penumbra seemed to colonize the pore area leading to its extinction. They found that the maximum value of the vertical component of the magnetic field $B_{v e r}$ in the pore was around $1.4 \mathrm{kG}$ and argued that a stable umbra-penumbra boundary could not be formed in that case because the pore did not fulfill the canonical critical value of $B_{\text {ver }}=1.8 \mathrm{kG}$, empirically found by Jǔrcák (2011) and Jurčak et al. (2015) to be a crucial value for the formation of a stable umbra-penumbra boundary in a magnetoconvective context. In our case, we do not have enough information on the magnetic field configuration in the pore during the formation process of the anomalous penumbra due to the lack of SP scans during those stages. Nonetheless, its evolution on the G-band images looks in some aspects similar to the case studied in Jurčak et al. (2017): the filaments seem to grow at the expense of the adjacent pore. However, an important difference is that in the case studied by Jurčak et al. (2017), the penumbra ends up as an orphan penumbra once the pore has disappeared, that is, the filaments are not connected to any umbral region, while the anomalous penumbra in our study is continually attached to the umbra of the main sunspot.

The evolution of the anomalous penumbra in AR 10930 is associated with high chromospheric activity. The study of the associated chromospheric and coronal dynamics, from the time of the AR's first appearance over the solar east limb on 2006-12-06 until the disappearance of the CEF on 2006-12-09 when the center-side penumbra shows the NEF only, might provide us with important information on how the anomalous penumbra was formed and how the CEF is maintained for a couple of days before reversing into a NEF. This will be the topic of future work.

\subsection{Conclusion}

In this paper, we report the observation of a red-shifted region in the center-side penumbra of the main sunspot of the AR NOAA 10930 on 2006-12-08 06:11:14 UT, at photospheric heights. This is, to our knowledge, a unique observation of a counter Evershed flow (CEF) at the photospheric heights covering a sizable part of the penumbra of a mature sunspot.

By using the SPINOR 2D code to invert spectropolarimetric data of the sunspot from the Hinode SOT/SP instrument, we investigated the characteristics of the CEF in the photosphere and have compared them with the physical properties of the normal Evershed flow (NEF) in the same sunspot. 
The results found here are consistent with both scenarios, namely that the temperature gradient or the magnetic field gradient is the main driver of both the normal Evershed flow and the anomalous or counter Evershed flow. This implies that we cannot distinguish between the convective driver and the siphon flow scenarios. However, this result suffers from the shortcomings of present observations and analysis techniques: the inability to peer below the solar surface and the lack of knowledge of the true geometrical height scale.

A comparison of our results with recent high-resolution sunspot simulations that display a NEF in the penumbra and a number of transient regions with a CEF at photospheric heights (see Rempel 2015) will provide us with additional tools to determine the dominant forces driving the flows ${ }^{4}$. An important advantage of this is that we will be able to study the dynamics of the flows at constant geometrical heights and to look into the vertical gradients at sub-photospheric depths, which are not accessible to observations.

Finally, studying in detail the history of the sunspot and the associated chromospheric activity can help us understand the formation process of the anomalous penumbra, the initiation of the CEF, and its change of direction into a NEF. This will be the topic of a future study.

\footnotetext{
${ }^{4}$ See Chapter 4.
} 



\section{Normal and counter-Evershed flows in sunspot MHD simulations ${ }^{1}$}

There have been a few reports in the literature of counter-Evershed flows observed in well developed sunspot penumbrae, i.e. flows directed towards the umbra along penumbral filaments. Here we investigate the driving forces of such counter-Evershed flows in a radiative magnetohydrodynamic simulation of a sunspot and compare them with the forces acting on the normal Evershed flow. The simulation covers a timespan of 100 solar hours and generates an Evershed outflow exceeding $8 \mathrm{~km} \mathrm{~s}^{-1}$ in the penumbra along radially aligned filaments where the magnetic field is almost horizontal. Additionally, the simulation produces a fast counter-Evershed flow (i.e., an inflow near $\tau=1$ ) in some regions within the penumbra, reaching peak flow speeds of $\sim 12 \mathrm{~km} \mathrm{~s}^{-1}$. The counter-Evershed flows are transient and typically last a few hours before they turn into outflows again. By using the kinetic energy equation and evaluating its various terms in the simulation box, we found that the Evershed flow occurs due to overturning convection in a strongly inclined magnetic field while the counter-Evershed flows can be well described as siphon flows.

\subsection{Introduction}

The origin of large-scale flows in the penumbra of sunspots is of particular interest in observational and theoretical studies of sunspots. The most prominent flow in photospheric penumbrae is the Evershed flow (EF; Evershed 1909), an almost horizontal and radially outward directed flow of plasma with speeds in the kilometers per second range (typical spatially averaged speeds being $1-2 \mathrm{~km} \mathrm{~s}^{-1}$ ). The nearly horizontal flow is usually subsonic, although supersonic flows have been observed (e.g., Wiehr 1995, del Toro Iniesta et al. 2001, Bellot Rubio et al. 2004, Borrero et al. 2005). The physical mechanism responsible for driving the EF is closely connected to the fine structure of the penumbra, which is manifested through the penumbral intensity, magnetic field and velocity structure (see, e.g., detailed reviews by Solanki 2003, Thomas and Weiss 2004, 2008, Borrero 2009, Scharmer 2009, Schlichenmaier 2009, Tritschler 2009, Bellot Rubio 2010, Borrero and Ichimoto 2011, Rempel and Schlichenmaier 2011). All these quantities display an almost radial filamentary structure in the penumbra. In particular, the magnetic field configuration comprises two major components, one containing generally stronger and more vertical fields (so-called spines) which is thought to be the result of a protrusion of the

\footnotetext{
${ }^{1}$ This Chapter is based on a manuscript published in The Astrophysical Journal (Siu-Tapia et al. 2018).
} 
umbral field into the penumbra (see, e.g., review by Borrero and Ichimoto 2011), and the second one being composed by weaker and more inclined fields (intra-spines, hereafter referred to as filaments) where the EF takes place (see, e.g., Tiwari et al. 2013). This configuration has been referred to as uncombed penumbra (Solanki and Montavon 1993) or interlocking-comb structure (Thomas and Weiss 1992).

Several models have been proposed to explain the filamentary nature of the penumbra, e.g., Danielson (1961), Meyer and Schmidt (1968b), Choudhuri (1986), Solanki and Montavon (1993), Schlichenmaier et al. (1998a,b), Thomas et al. (2002), Spruit and Scharmer (2006), Scharmer and Spruit (2006). However, not all the models contain a self-consistent description of the EF. Some models based on stationary magnetic flux tubes representing the filaments, describe the EF as a siphon flow driven by a gas pressure difference between the footpoints of the flux tube (e.g., Thomas and Montesinos 1993). On the other hand, the dynamic magnetic flux tube model presented by Schlichenmaier et al. (1998a) produces an EF as a combination of hot plasma rising at the inner footpoint of the tube and a radial acceleration driven by a pressure gradient, consequence of radiative losses at the surface. There is also the model of Scharmer and Spruit (2006) that says that the EF takes place in field-free intrusions. More recently, numerical magnetohydrodynamics (MHD) simulations have succeeded in reproducing the EF as a result of overturning convection in the presence of an inclined magnetic field (e.g., Heinemann et al. 2007, Scharmer et al. 2008, Rempel et al. 2009a,b, Kitiashvili et al. 2009, Rempel 2011, 2012a). The EF is in these cases interpreted as the convective flow component in the direction of the magnetic field. In these models, the penumbral fine structure results from anisotropic magnetoconvection.

During the early stages of penumbrae formation, line-of-sight (LOS) velocities of opposite sign with respect to that displayed by the typical EF have been reported by Schlichenmaier et al. (2012) and Romano et al. (2014). This has been interpreted as inflows towards the pore before the formation of the penumbra. On rare occasions, welldeveloped penumbrae can also harbor counter-EF (inflows) at the photosphere (Kleint and Sainz Dalda 2013, Louis et al. 2014, Siu-Tapia et al. 2017). In particular, Siu-Tapia et al. (2017) reported the observation of a prominent counter-EF with a lifetime of $\sim 2$ days in the disk center-side of a well-developed penumbra. The counter-EF showed considerable fine structure, i.e., the counter-EF was confined along "reversed" penumbral filaments, with their heads/sources located at the outer penumbral boundary and their tails/sinks observed at the inner penumbral edge. Siu-Tapia et al. (2017) showed that, as in the normal-EF, the filaments carrying the counter-EF display temperature and magnetic field gradients that are both consistent with the direction of the flow, being compatible with both the magneto-convective driver scenario as well as the siphon flow mechanism.

In this work, we analyze the results of a MHD high-resolution sunspot simulation by Rempel (2015), which produces a penumbra with normal-EF (outflows) as well as a fast counter-EF (inflows) in some parts of the penumbra at photospheric heights, with lifetimes of several hours. We investigate and identify the driving forces acting on both, the normal- and the counter-EF. 


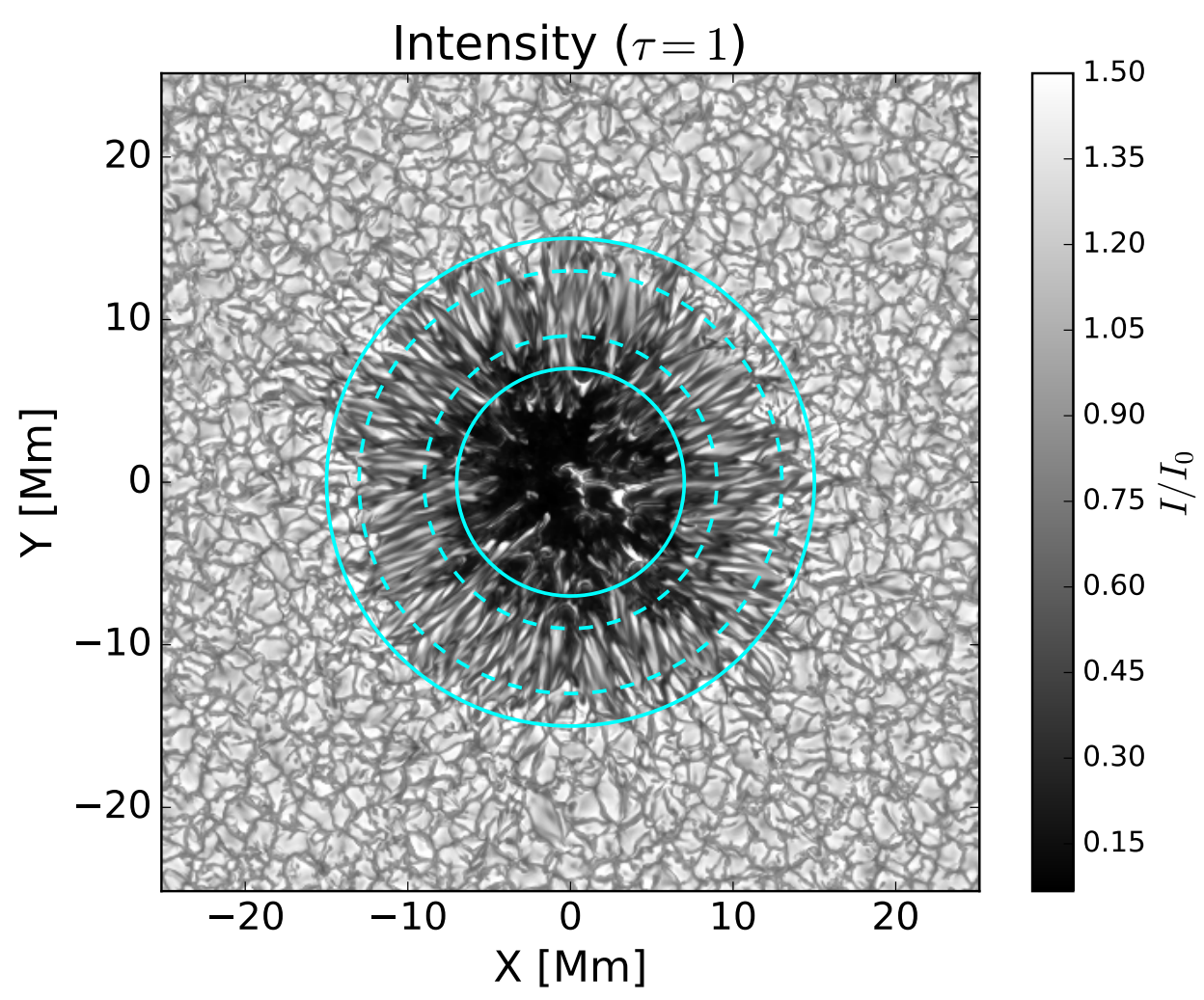

Figure 4.1: Intensity image of the simulated sunspot, relative to the average quiet sun intensity $I_{0}$, at about 67 hours after initialization of the simulation run. The image corresponds to the full-spatial resolution series and shows the innermost $50.2 \times 50.2 \mathrm{Mm}^{2}$ of the simulation domain. Solid circles have been placed at radial distances of $7 \mathrm{Mm}$ (where the azimuthally averaged intensity reaches $0.45 I_{0}$ ) and $15 \mathrm{Mm}$ (where the azimuthally averaged intensity reaches $0.9 I_{0}$ ) from the center of the sunspot, delimiting the inner and outer penumbra, respectively. The dashed circles at $R=9$ and $13 \mathrm{Mm}$ delimit the middle penumbra.

\subsection{Simulation}

We use the 3D high-resolution sunspot simulation setup described in detail by Rempel (2015) and used therein to study the role of the penumbra and associated flows for sunspot decay. The simulation is based on the MURaM radiative MHD code (Vögler et al. 2005, Rempel et al. 2009b, Rempel 2014) and covers a timespan of 100 solar hours. An animation covering $25 \mathrm{hr}$ (from $t=50$ to $75 \mathrm{hr}$ after the initialization of the simulation) is provided as part of the online material of Rempel (2015).

As described in Rempel (2015), the sunspot setup used open boundary conditions that do not maintain the initial field structure against decay driven by convective motions. The simulation initial state was a relaxed small scale dynamo, the sunspot simulation was then initialized by inserting an axisymmetric self-similar magnetic field structure into the domain with total initial flux of $9 \times 10^{21} \mathrm{Mx}$, a field strength at the bottom of the domain of around $20 \mathrm{kG}$ and at the top of the domain of around $3 \mathrm{kG}$. The simulated sunspot had an almost constant magnetic flux for the simulated timespan of 100 hours and used a 


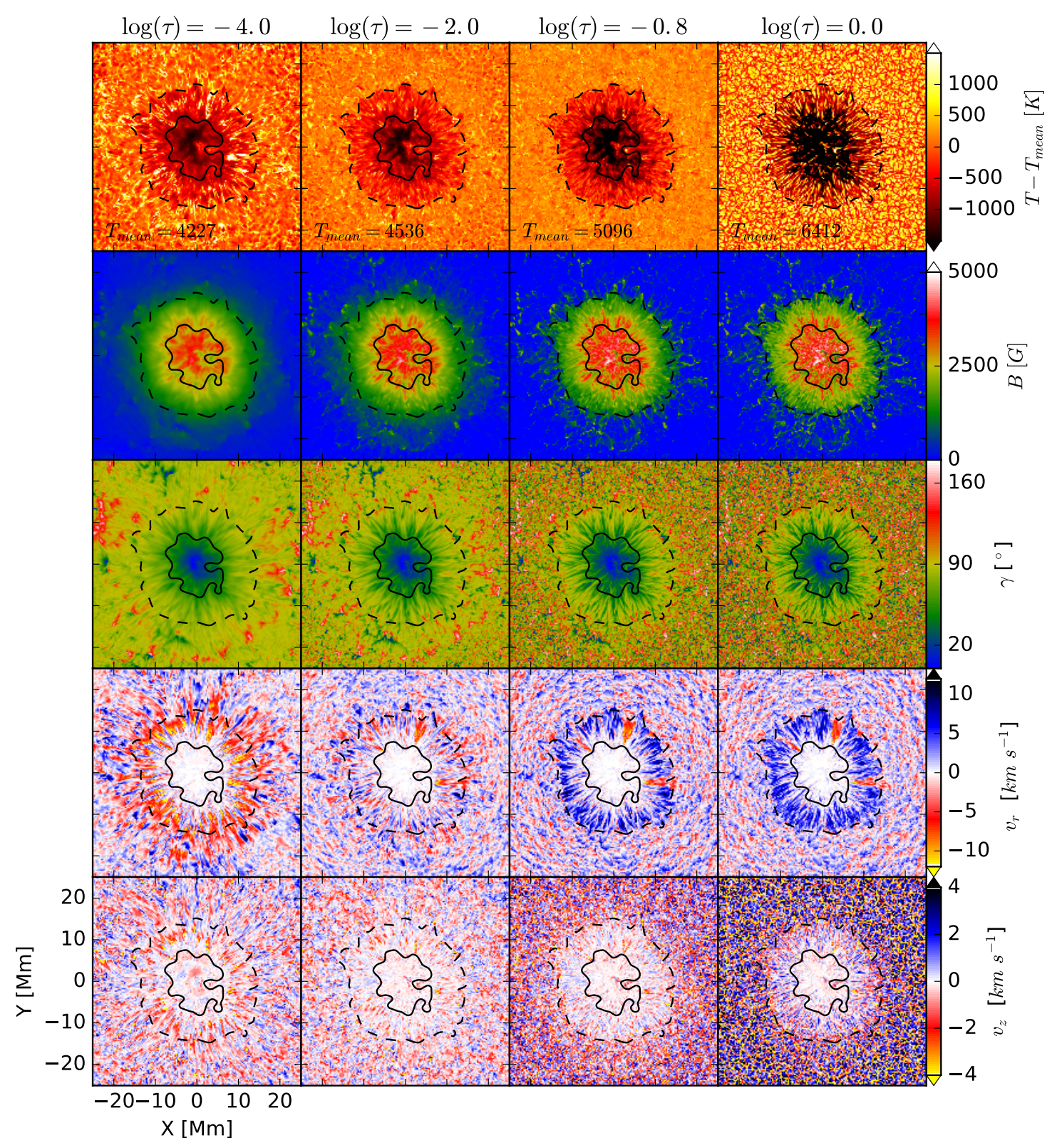

Figure 4.2: Fine structure of the sunspot at different optical depth levels. From left to right: $\log (\tau)=-4,-2,-0.8$ and 0 . From top to bottom: Temperature perturbation, $T-$ $T_{\text {mean }}[\mathrm{K}]$; magnetic field strength, $B[\mathrm{G}]$; field inclination, $\gamma\left[{ }^{\circ}\right]$ with respect to vertical; radial flow velocity, $v_{r}\left[\mathrm{~km} \mathrm{~s}^{-1}\right]$; and vertical flow velocity, $v_{z}\left[\mathrm{~km} \mathrm{~s}^{-1}\right]$. A field inclination of $0^{\circ}$ corresponds to vertical field with the same polarity as the umbra, $90^{\circ}$ to horizontal, and $180^{\circ}$ to vertical field with opposite polarity of the umbra. Positive $v_{r}$ and $v_{z}$ values (blue-to-black colors) indicate outflows and upflows, respectively. Note that this sign convention differs from the one used in observational studies, which normally follow the spectroscopic definition of flow velocities along the line of sight, where negative values denote flows moving towards the observer and positive values flows moving away from the observer. Black contour lines indicate the regions where the intensity $I<0.45 I_{0}$ and $I<0.9 I_{0}$. The images correspond to the same snapshot as Figure 4.1. 
magnetic top boundary condition that imposes a sufficiently horizontal field to maintain a penumbra.

After $t=50$ hours, the simulated sunspot showed well-developed penumbral fine structure (see, e.g., Figure 4.1 for an intensity image obtained about 67 solar hours after initialization of the simulation), i.e., radially aligned filaments with close to horizontal field (see Figure 4.2). Along these filaments, there are fast radial outflows reaching peak flow velocities of $\sim 12 \mathrm{~km} \mathrm{~s}^{-1}$. In addition to these outflow regions, there are also some patches in the penumbra that have a counter-EF (inflows in the photosphere). These regions are transient, lasting a few hours before they turn into outflows again.

To investigate the nature of the counter-EF, we analyze the simulation time-steps from 60 to 70 solar hours (The range of time during which the counter-EF are found in the simulations). As described in Rempel (2015), the photosphere is located about $700 \mathrm{~km}$ beneath the top boundary, and the simulation domain extends about $18 \mathrm{Mm}$ in depth below the photosphere and $98 \mathrm{Mm}$ horizontally, using a grid spacing of $24 \mathrm{~km}$ vertically and 48 $\mathrm{km}$ horizontally, which is required to capture penumbral fine structure and the Evershed flow. The simulation ran on a $2048 \times 2048 \times 768 x y z$-grid (in the following presentation the $z$-direction is vertical). Full resolution data cubes were written every 4500 seconds, whereas data cubes at half the spatial resolution were written every 900 seconds. A nongray run was restarted from one of the full resolution cubes and evolved for 5000 time steps, i.e. 1125 seconds. All of the snapshots have improved numerics to address the drift problem described in Rempel (2015), related to numerical diffusion and need for $\nabla \cdot B$ cleaning.

A full-spatial-resolution snapshot obtained with non-gray radiative transfer $(t \sim 67$ solar hours) is used in this work to study the filamentary structure of the normal- and the counter-EF. The half-resolution data cubes obtained with gray radiative transfer are employed to analyze the driving of the flows in the penumbra and their evolution in time.

\subsection{Results}

\subsubsection{Filamentary structure of the penumbra}

Figure 4.1 shows the normalized intensity of the simulated sunspot at $\tau=1$ for a snapshot at $t \sim 67$ solar hours. Here we are mainly interested in the penumbra, which appears to have a uniform filamentary structure in intensity at $\tau=1$.

Figure 4.2 displays the filamentary fine structure of the penumbra as seen in the different physical quantities and at different optical depths: $\log (\tau)=-4,-2,-0.8$ and 0 . The filamentary structure of the penumbra is more evident at $\log (\tau)=0$, where the penumbral filaments appear as bright (hot) elongated channels with magnetic fields that become more inclined from the inner penumbral edge outwards, and return back to the interior $\left(\gamma>90^{\circ}\right)$ towards the outer penumbral edge. Also, the velocity vector in the penumbral filaments is mostly radial.

The radial velocity maps in Figure 4.2 (fourth row) show that the penumbra is dominated by an inflow with respect to the center of the spot (red-to-yellow colors) at $\log (\tau)=$ -4.0, which resembles the chromospheric inverse Evershed flow (IEF) (Dialetis et al. 1985 ) but occurs at lower heights, and by an outflow below $\log (\tau)=-2.0$ (blue-to-black 
colors), which represents the photospheric normal-EF. However, there are also some regions within the penumbra at $\log (\tau)=-2.0,-0.8$, and 0 where the radial velocity is negative, indicating photospheric counter-EF. These photospheric inflows occur along penumbral filaments. Their associated vertical velocity shows large negative values (downflows) close to the inner penumbral boundary, at the end of these inflow filaments.

In order to get insight about the physical differences between the regions harboring the counter-EF (CEF) and the regions with the normal-EF (NEF), in Figure 4.3 we compare the characteristics of the gas and the magnetic field along the central axes of two radial flow channels which carry a CEF and a NEF respectively. Figure 4.3 shows a vertical cross section along the normalized central axes of the two selected penumbral filaments, a CEF-carrying filament (left panels) and a NEF-carrying filament (right panels), from $z=-600 \mathrm{~km}$ to $200 \mathrm{~km}$ above the surface (which is defined as the average height of the $\tau=1$ level in the quiet Sun). The selected individual filaments are representative of each filament type (CEF-carrying and NEF-carrying, respectively) since most of the qualitative physical characteristics discussed below are true for most of the filaments in this snapshot.

The velocity panels in Figure 4.3 show the sources of the NEF, i.e. the portion of the filament on the right panels that harbors both upflows $\left(v_{z}>0\right)$ and outflows $\left(v_{r}>0\right)$, spanning around $85 \%$ of the filament's normalized length, while the sinks (regions where $v_{z}<0$ and $v_{r}>0$ ) are observed towards the outermost $15 \%$ of the filament's length. In contrast, the sources of the CEF (regions where $v_{z}>0$ and $v_{r}<0$ on the left panels) are located in the outer endpoint of the filament (i.e., in the endpoint closer to the quiet Sun) from $\sim 0.6$ to 1 in the normalized length scale, while their sinks (regions where $v_{z}<0$ and $v_{r}<0$ ) dominate mainly within the innermost $30 \%$ of the filament. Therefore, unlike the CEF-carrying filament, almost the entire NEF-carrying filament behaves as a source of flow.

Nonetheless, there are also some similarities. In both the CEF and NEF flow channels, the upflow cells harbor hot gas $(T>10000 \mathrm{~K})$ and are located in regions where the magnetic field is relatively weak. Also, the magnetic field in the upflow cells becomes more inclined with height and in the direction of the flows (see arrows on $v_{z}$ and $B$ panels in Figure 4.3). Furthermore, in both the NEF and CEF, the gas flows with supersonic speeds in the sink regions (regions where $v_{z}<-8 \mathrm{~km} \mathrm{~s}^{-1}$ are enclosed by white contour lines on $v_{z}$ and $T$ panels in Figure 4.3, with the sound speed being close to $6 \mathrm{~km} \mathrm{~s}^{-1}$ near the $\tau=1$ surface), and exhibits lower temperatures than at the sources $(T<8000 \mathrm{~K})$. However, the downflowing gas in the sink regions are slightly warmer than their surroundings, possibly as a result of the formation of shocks, since the downflow speeds are generally supersonic at those places, but the downflow speeds suddenly drop with depth from supersonic to subsonic, indicating that deceleration takes place in the form of shocks in both cases, NEF and CEF.

Compared to the field strength at the source regions $(B<2000 \mathrm{G})$ in both, CEF and NEF, the magnetic field presents a strengthening at the sink regions $(B>2500 \mathrm{G})$ where it gradually becomes more vertical in the flow direction. Furthermore, in both NEF and CEF, the magnetic field polarity in the sink regions is opposite to that at their corresponding sources. Because in Figure 4.3 we concentrate on the central axes of the filaments, we see only the 'end of filament' sinks. Nonetheless, most of the overturning mass flux goes to the lateral sinks, so the end of filament sinks represent only a part of the picture since a significant fraction $(\sim 50 \%)$ of the returning mass flux is found in regions 


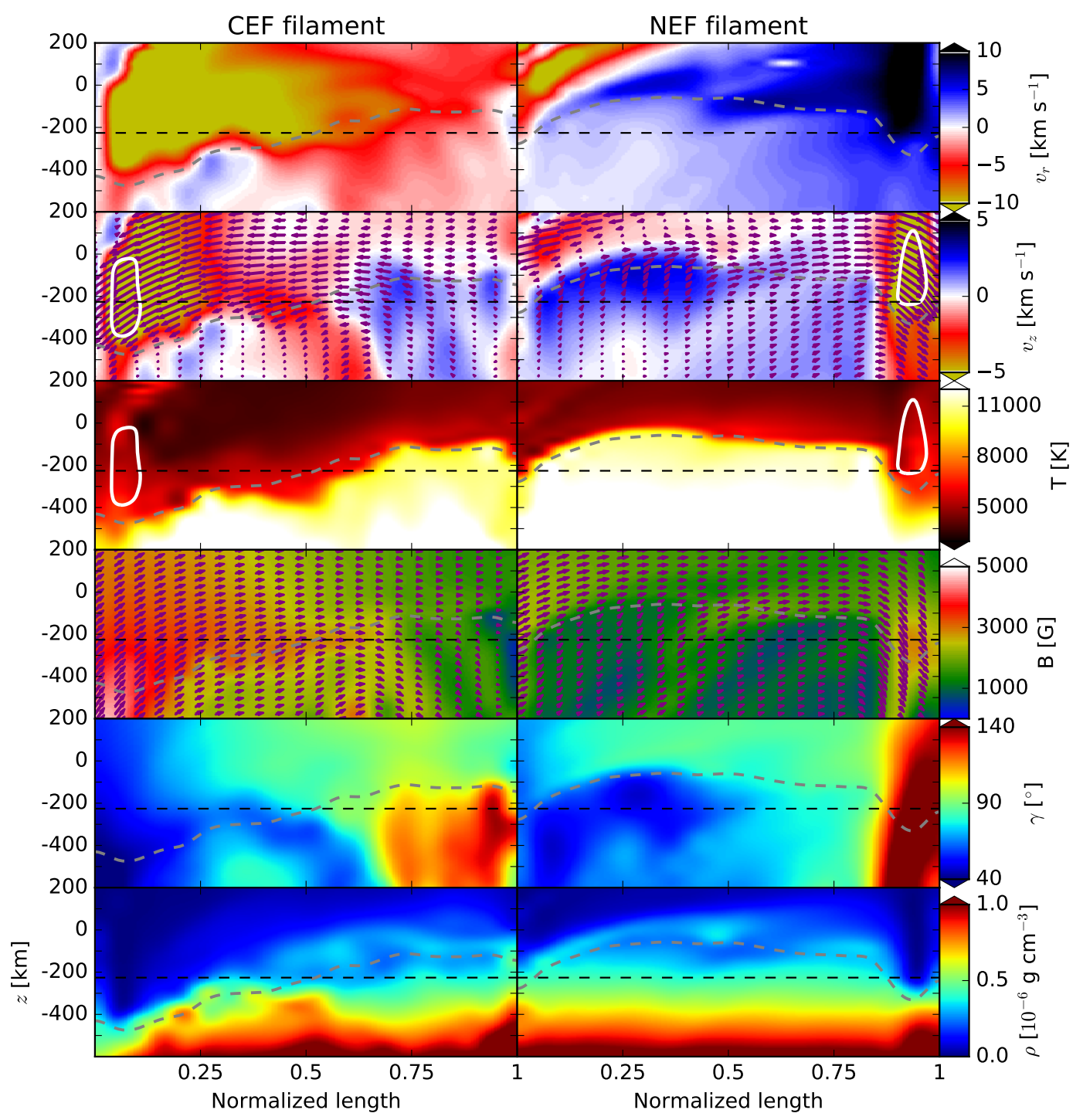

Figure 4.3: Vertical cross section through the central axes of two filaments, reaching from $-600 \mathrm{~km}$ to $200 \mathrm{~km}$ above the surface: (left) CEF-carrying filament and (right) NEFcarrying filament. The filaments were selected from the snapshot at $t \sim 67$ solar hours, which corresponds to a time when the CEF is prominent and stable. Panels show, from top to bottom: radial flow velocity $v_{r}$, vertical flow velocity $v_{z}$, temperature $T$, magnetic field intensity $B$, field inclination with respect to vertical $\gamma$, and density $\rho$. Gray dashed lines indicate the $\tau=1$ levels. Horizontal dashed lines are placed at $z=-226 \mathrm{~km}$, which is the average height of $\tau=1$ in the penumbra with respect to the quiet Sun. The arrows in the $v_{z}$ and $B$ maps indicate the direction of the flow and magnetic field, respectively, mainly to guide the eye. The white contour lines on the $v_{z}$ and $T$ maps indicate regions with supersonic downflows, i.e. where $v_{z}<-8 \mathrm{~km} \mathrm{~s}^{-1}$. As a reference, the umbra is located to the left. 
with still upward pointing field when the lateral sinks are included.

Dashed gray lines in Figure 4.3 indicate the variation in height of the $\tau=1$ levels along the two different flow channels with respect to the reference penumbral average height $z=-226 \mathrm{~km}$ (horizontal black dashed lines). Such constant optical depth levels are depressed in the downflowing part of the filaments and are elevated in the upflowing part of the filaments. The depression of the constant optical depth surfaces in the sink regions are caused by lower temperatures and densities of the downflowing gas compared to the upflowing gas. This is an important aspect to consider in observational studies of penumbral filaments, in which the dynamics of the flows and the magnetic field structure are only accessible at constant optical depth surfaces, see also discussion in van Noort et al. (2013). In this simulation, the magnetic field strength suffers a large enhancement in the downflowing part of the filaments (sinks) along the $\tau=1$ level, taking values of up to $\sim 5 \mathrm{kG}$ in the CEF filament, and $\sim 2.5 \mathrm{kG}$ in the NEF-carrying filament. For the CEF filaments, such a very large strengthening of the field is partly the result of the strong depression of the $\tau=1$ level at the sinks and their close vicinity to the umbral field (note that the CEF sinks are mainly located close to the umbral boundary). The strengthening of the field at the sinks of the CEF filaments at constant geometrical height also contributes (as can be seen in the left column of Figure 4.3). Moreover, the sinks of the NEF filaments also present a field intensification near the $\tau=1$ level. Such local field strengthening might be produced by the supersonic downdrafts of magnetized flux concentrations at the sink regions as suggested by van Noort et al. (2013) for explaining the observation of field strengths of the order of $7 \mathrm{kG}$ in supersonic downflow regions at the outer penumbra of a sunspot.

\subsubsection{Driving forces of the penumbral flows}

Our following analysis follows very closely the analysis performed by Rempel (2011) aimed to investigate the physical processes that lead to the driving of large-scale outflows around sunspots. Therefore, in order to investigate which forces are responsible for driving the inflows and the outflows in the penumbra, we analyze the various terms in the kinetic energy equation used by MURaM, which is derived from the momentum equation. Since we concentrate on time averages within this section, we use the following energy balance equation, which neglects the temporal derivatives and assumes stationary flows:

$$
\underbrace{\vec{v} \cdot(\rho \vec{g}-\nabla p)}_{\text {Pressure }}+\underbrace{\vec{v} \cdot(\vec{j} \times \vec{B})}_{\text {Lorentz }} \underbrace{-\rho \vec{v} \cdot[(\vec{v} \cdot \nabla) \vec{v}]}_{\text {Acceleration }}+\underbrace{\vec{v} \cdot F_{\text {visc }}}_{\text {Viscosity }}=0
$$

In this energy balance equation, a negative acceleration term represents a source of kinetic energy given that, under the assumption of stationarity, the acceleration term is identical to the negative divergence of the kinetic energy flux, $\rho \vec{v} v^{2} / 2$.

We compute the individual terms in the energy balance equation as follows:

$$
\begin{gathered}
\left\langle P_{i}^{ \pm}\right\rangle=\left\langle v_{i}^{ \pm}\left[\rho g_{i}-(\nabla p)_{i}\right]\right\rangle \\
\left.\left\langle L_{i}^{ \pm}\right\rangle=\left\langle v_{i}^{ \pm}(\vec{j} \times \vec{B})_{i}\right]\right\rangle \\
\left\langle A_{i}^{ \pm}\right\rangle=\left\langle-\rho v_{i}^{ \pm}[(\vec{v} \cdot \nabla) \vec{v}]_{i}\right\rangle
\end{gathered}
$$


The viscosity term is not explicitly calculated but instead we use an approximated magnitude which we call residual force:

$$
\left\langle R_{i}^{ \pm}\right\rangle=\left\langle-\left(P_{i}^{ \pm}+L_{i}^{ \pm}+A_{i}^{ \pm}\right)\right\rangle
$$

In the equations 4.2-4.5, $i$ indicates the Cartesian coordinates and the brackets indicate temporal averages over 10 hours, from $t=60-70$ hours after initialization of the simulation. We then use the transformation to cylindrical coordinates to separate the directions along and perpendicular to the penumbral filaments, $r$ and $z$ coordinates, respectively. With $v_{i}^{ \pm}$denoting positive and negative velocity components respectively $\left(v_{r}^{+}\right.$represents outflows and $v_{z}^{+}$upflows).

The top panels of Figure 4.4 show the azimuthally and temporally averaged vertical slices of the radial velocity separately for all the inflows (left) and all the outflows (right) in the penumbra, i.e. using masks that select only the grid points where $v_{r}<0$ and $v_{r}>0$ respectively, from $z=0.5$ to $-3 \mathrm{Mm}$ (relative to the average height of the $\tau=1$ level in the quiet Sun). NEF-related outflow speeds larger than $2 \mathrm{~km} \mathrm{~s}^{-1}$, and in some places even larger than $5 \mathrm{~km} \mathrm{~s}^{-1}$, stand out close to the $\tau=1$ level, being generally restricted to below $\tau=0.01$. Note that in this case the mask excludes any inflow occurring in the penumbra, even those corresponding to the IEF-like feature which dominates the penumbra above $\tau=0.01$ (see radial velocity maps in Figure 4.2). In contrast, the inflow speeds above $2 \mathrm{~km} \mathrm{~s}^{-1}$, which also stand out close to the $\tau=1$ level due to the CEFs, produce a continuously increasing inflow profile towards the higher layers due to the inclusion of all those inflows in the IEF-like feature that peak above $\tau=0.01$. It is not in the scope of the present work to analyze such an IEF-like feature. Moreover, given that these inflows occur close to the top boundary of the simulation box, which is numerically closed, they likely are highly influenced by the upper boundary conditions.

Figure 4.4 also shows the resultant radial energy conversion terms in the penumbra, separated for all inflows $\left(\left\langle A_{r}^{-}\right\rangle,\left\langle L_{r}^{-}\right\rangle\right.$and $\left\langle P_{r}^{-}\right\rangle$, plots on the left) and for all outflows $\left(\left\langle A_{r}^{+}\right\rangle\right.$, $\left\langle L_{r}^{+}\right\rangle$and $\left\langle P_{r}^{+}\right\rangle$, plots on the right). Recall that these energy conversion profiles show the 10 hours average of the azimuthally averaged terms in each mask. Negative values of $A_{r}^{-}$and $A_{r}^{+}$indicate inward and outward acceleration of the fluid, respectively. Likewise, positive values of $P_{r}^{-}$and $L_{r}^{-}$represent a source of kinetic energy for inflows, whilst positive values of $P_{r}^{+}$and $L_{r}^{+}$represent an energy source for outflows.

Panels in Figure 4.5 are in the same format as panels in Figure 4.4 and show the vertical energy conversion terms $\left(\left\langle A_{z}^{+}+A_{z}^{-}\right\rangle,\left\langle L_{z}^{+}+L_{z}^{-}\right\rangle\right.$and $\left.\left\langle P_{z}^{+}+P_{z}^{-}\right\rangle\right)$associated to the inflows (panels on the left) and to the outflows (panels on the right). There, negative values of $A_{z}^{+}+A_{z}^{-}$indicate vertical acceleration of the fluid in inflow and outflow regions, accordingly.

While most of the radial acceleration of the fluid takes place within the inner and middle penumbra for the outflows (negative values of $\left\langle A_{r}^{+}\right\rangle$in Figure 4.4), the inflows are predominantly accelerated within the middle and outer penumbra (negative values of $\left.\left\langle A_{r}^{-}\right\rangle\right)$.

These regions are confined to a narrow layer near the $\tau=1$ level. There, the Lorentz force is the primary driver of the outflows, while the pressure terms have a weakly negative energetic contribution. In contrast, the inflows are driven mainly by the radial pressure forces, while the radial Lorentz force shows a negative contribution on average. However, plots in Figure 4.6 reveal that the radial Lorentz term contributes mostly positively close 

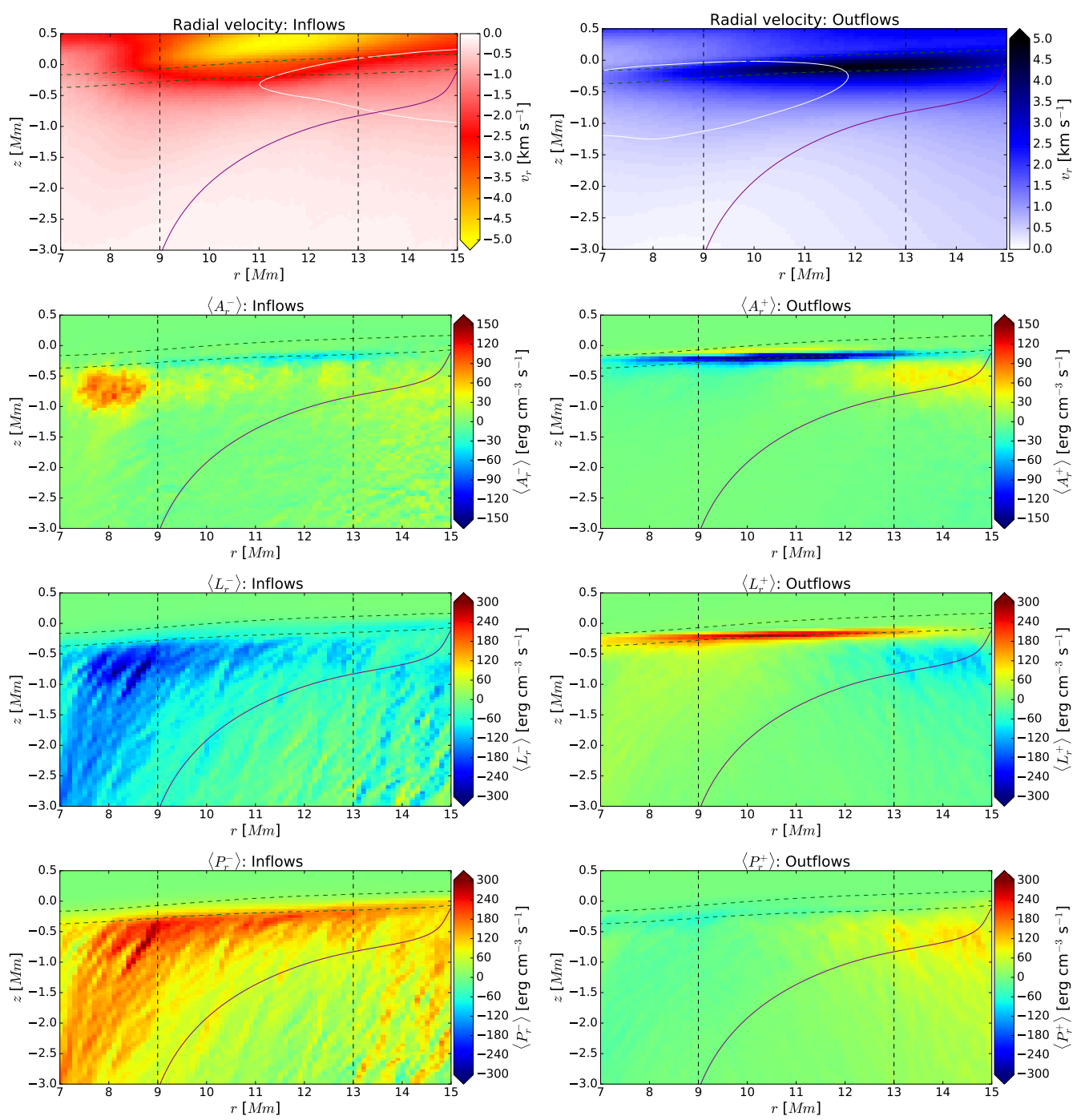

Figure 4.4: Radial flow velocity and radial energy conversion terms as functions of radius and height, using masks that select only inflows $\left(v_{r}<0\right.$, left plots) and only outflows $\left(v_{r}>0\right.$, right plots) in the penumbra. All plots were averaged from 60 to 70 solar hours using the half spatial resolution $3 \mathrm{D}$ cubes with temporal cadence of $900 \mathrm{sec}$. From top to bottom: radial flow velocity, radial components of the acceleration, Lorentz and pressure forces. The residual terms in both cases are negligible. Vertical dashed lines delimit the inner penumbra ( $r=7-9 \mathrm{Mm}$ from the center of the sunspot), the middle penumbra $(r=9-13 \mathrm{Mm})$ and the outer penumbra $(r=13-15 \mathrm{Mm})$ as indicated by the cyan circles in Figure 4.1. Green dashed lines indicate the averaged $\tau=1$ and $\tau=0.01$ levels. The purple curve is an azimuthally and temporally averaged iso-contour placed at $B=950 \mathrm{G}$ and used as reference to distinguish between the high- and low-field regions in the penumbra. White contour lines on the top plots enclose regions with vertical upflow speeds larger than $0.3 \mathrm{~km} \mathrm{~s}^{-1}$ when using masks that select only the sources of inflows (i.e. grid-points where $v_{r}<0$ and $v_{z}>0$, left) and the sources of outflows (i.e. grid-points where $v_{r}>0$ and $v_{z}>0$, right). 

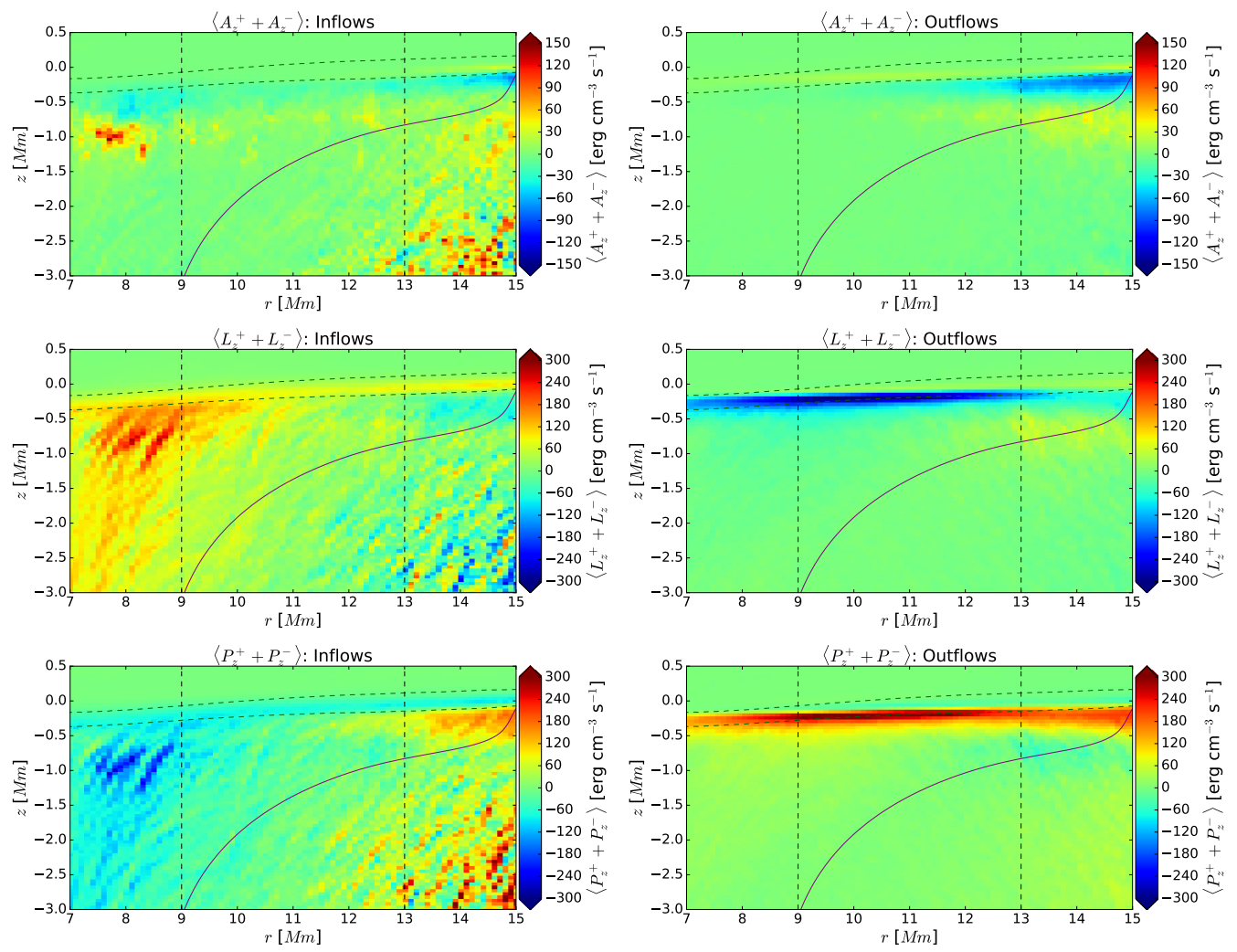

Figure 4.5: Vertical energy conversion terms as functions of radius and height in the penumbra (azimuthal and temporal averages). From top to bottom: $\left\langle A_{z}^{+}+A_{z}^{-}\right\rangle,\left\langle L_{z}^{+}+L_{z}^{-}\right\rangle$, and $\left\langle P_{z}^{+}+P_{z}^{-}\right\rangle$in inflow regions (panels on the left) and in outflow regions (panels on the right). Same format as plots in Figure 4.4.

to $\tau=1$ when the masks only include the regions responsible for driving inflows (i.e. source regions where both $v_{r}<0$ and $v_{z}>0$ ), and neglect those regions where the inflows sink (i.e. regions where $v_{r}<0$ and $v_{z} \leq 0$ ).

Plots in Figure 4.4 also show that in deeper layers the radial pressure terms are almost in balance with the radial Lorentz terms, resulting in only minor acceleration of outflows and inflows in the deep penumbra. Likewise, almost no driving forces exist above $\tau=0.01$. Towards the inner penumbra for the inflows and outer penumbra for the outflows, the layers below the $\tau=1$ level display a similar forcing pattern, though the energy conversion is on average larger at the inner penumbra for inflows than at the outer penumbra for outflows. In both cases, from $\tau=1$ down to $z \sim-1 \mathrm{Mm}$, the radial Lorentz term is overcompensating the radial pressure driving, producing a radial deceleration of inflows and outflows (positive values of $\left\langle A_{r}^{-}\right\rangle$and $\left\langle A_{r}^{+}\right\rangle$, respectively). This energy is however transferred into a vertical acceleration of the fluid (negative values of $\left\langle A_{z}^{+}+A_{z}^{-}\right\rangle$in Figure 4.5). Such vertical acceleration is mainly downwards at the inner penumbra for inflows and at the outer penumbra for outflows, i.e. negative values of $A_{z}^{-}$dominate in the $\left\langle A_{z}^{+}+A_{z}^{-}\right\rangle$average profiles at those places. Finally, the downflowing gas is decelerated again close to $z=-1 \mathrm{Mm}$. This is seen as the change into positive values of $\left\langle A_{z}^{+}+A_{z}^{-}\right\rangle$in Figure 4.5. In the case of the outflows, a transition towards "normal" overturning convection (i.e. more granular-like upflows and downflows where vertical pressure forces lead 

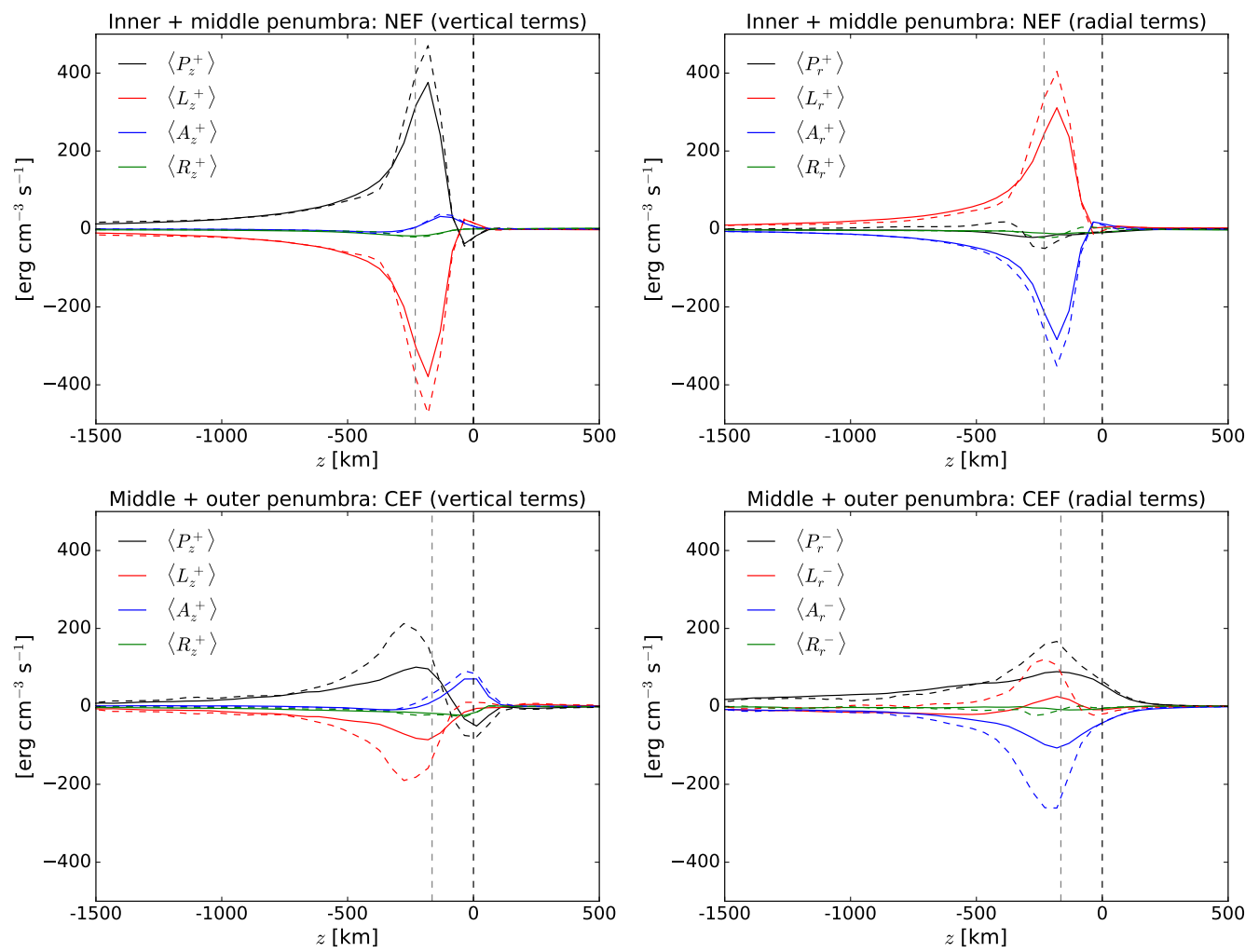

Figure 4.6: Height dependence of the energy conversion terms in the kinetic energy equation. The solid curves show the energy terms averaged in time (from 60 to $70 \mathrm{hr}$ ) and at each height for the regions that have both upflows and outflows $\left(v_{z}>0\right.$ and $v_{r}>0$, upper plots) and for the regions with both, upflows and inflows ( $v_{z}>0$ and $v_{r}<0$, lower plots). The dashed curves show the averaged energy terms when using a mask that extracts the penumbral filaments carrying the $\mathrm{CEF}$ and the NEF, correspondingly, and neglects the contribution of all other flows (e.g. those occurring beyond the reference iso-contour at $B=950 \mathrm{G}$ shown in Figures 4.4 and 4.5). We considered only flows in the inner and middle penumbra for the plots on the top, and in the middle and outer penumbra for the plots on the bottom. The energy conversion terms are separated into their vertical components (left plots) and radial components (right plots). Black: pressure forces, red: Lorentz forces, blue: acceleration forces, green: residual forces. The vertical dashed lines are placed at $z=0 \mathrm{~km}$ (black) and at the average height of the $\tau=1$ level in the corresponding regime(s) of the penumbra, which are indicated at the headers of each plot. 
mostly to vertical acceleration) starts taking place in the region $r>13 \mathrm{Mm}$. This also contributes to the strong positive $\left\langle A_{z}^{+}+A_{z}^{-}\right\rangle$found right below $\tau=1$, which also coincides with positive $\left\langle P_{z}^{+}+P_{z}^{-}\right\rangle$.

As mentioned above, most of the radial acceleration occurs from the middle to the outer penumbra in inflows, and from the inner to the middle penumbra in outflows. Such penumbral regimes also correspond to the average location of the respective flow source regions (see white contour lines in Figure 4.4). Therefore, plots in Figure 4.6 (solid curves) have been created by using a mask that specifically selects the sources of the outflows (regions where $v_{z}>0$ and $v_{r}>0$ ) within the inner and middle penumbra at each height, and the sources of the inflows (regions where $v_{z}>0$ and $v_{r}<0$ ) within the middle and outer penumbra at each height. The average depth profile of the energy conversion terms at the sources of each flow are then averaged in time (10 hours). Plots in Figure 4.6 show that most of the energy conversion takes place between $z=-500$ and $0 \mathrm{~km}$, and represents the driving of the NEF and the $\mathrm{CEF}$, correspondingly.

However, the solid curves in Figure 4.6 stand for the sources of everything that is flowing outwards (upper plots) and the sources of everything that is flowing inwards (lower plots) in the respective penumbral regimes. This includes the filaments of interest carrying the NEF and the CEF respectively, but there are also other and smaller scale flows that do enter the average. The weight or contribution of such flows to the average energy conversion terms seems to be negligible below $z \sim-500 \mathrm{~km}$ (by comparing with the dashed curves in Figure 4.6) but is larger in the near surface layers (between $z=-500 \mathrm{~km}$ and $z=0$ ) where the driving of the NEF and the CEF occurs. However, those additional flows do not modify the average energy balance of the NEF. Only in the radial energy balance of the CEF we see an increased contribution of the radial Lorentz term to the inward acceleration of the fluid when neglecting other inflows. This is because those additional inflows are mainly driven by radial pressure forces in regions where $L_{r}^{-}$is generally null or slightly negative.

In both, NEF and CEF sources, the upflow components show an approximate balance between $P_{z}^{+}$and $L_{z}^{+}$, mainly below and close to $\tau=1$. Higher up, the vertical pressure driving, which includes the contribution of gravity, changes sign and $A_{z}^{+}$becomes positive just below $z=0$, implying upflows that decelerate before reaching the photosphere. The steepening of $P_{z}^{+}$in the near surface layers is caused by the presence of strong magnetic fields. However, the field strength is about a factor 2-3 weaker in the outer penumbra than in the inner penumbra, which explains why the energy conversion is lower at the sources of the CEF than at the sources of the NEF, and might be related to the transient and unstable aspects of the CEFs.

For the NEF, we find that the energy extracted by the Lorentz force in the vertical direction, $L_{z}^{+}$, is almost completely transferred to the radial outward acceleration of the fluid, $A_{r}^{+}$, similar to the findings of Rempel (2011). The energy conversion by radial Lorentz force, $L_{r}^{+}$, is mainly balanced by $A_{r}^{+}$, and $P_{r}^{+}$has a slightly negative contribution close to $\tau=1$. This can be thought as a deflection of the vertical pressure driving by the Lorentz force $\left(v_{z} B_{r} \approx v_{r} B_{z}\right)$, i.e. the magnetic field forms an almost 90 degree nozzle connecting the pressure driving in the vertical direction with a radial outward acceleration. The Lorentz force does not do any net work. The ultimate energy source is the pressure force due to a large vertical pressure gradient which steepens in the near surface layers.

In normal convection, a loss of buoyancy of the gas near $\tau=1$ causes a deceleration 
of the upflows and, as the gas cannot go any higher, it is pushed horizontally to all sides by the rising gas underneath it (i.e. the decelerating gas builds up excess pressure which drives horizontal flows). In the NEF, there is also a loss of buoyancy of the gas near $\tau=1$, but in this case the horizontal pressure gradients only play a role in the azimuthal direction (i.e. to drive the horizontal lateral flows) and have slightly negative contribution in the radial direction. For the NEF, it is the inclined magnetic field in the penumbra who plays the most important role. Because in the penumbra there is a magnetic field pointing mainly in one direction, the gas motion loses degrees of freedom and therefore it moves in a preferred direction (the radial one). But the field is not the main accelerating agent of the gas flow in the radial direction, i.e. the density of the gas decreases at the surface where it is deflected radially, and because of mass flux conservation, its speed must be higher. The gas accelerates outwards because more and more gas is coming from below all along the filament, i.e. mass conservation requires the gas to move faster and faster horizontally. While in granulation the horizontally flowing gas can carry mass away on the horizontal plane isotropically, in the penumbra there are less degrees of freedom for the gas motion due to the magnetic structure, and therefore the horizontal flows must be faster in penumbra than in granules to carry away the same amount of mass (assuming equally strong upflows and equal horizontal size of upflowing patches). However, the existence of downflows at the sides of the filaments also removes part of the horizontally flowing mass, therefore limiting the speed of the radial flows.

In contrast, the CEF is predominantly driven by radial pressure forces (and so are all inflows at all heights). There is an additional positive contribution from the radial Lorentz term close to $\tau=1$, and the combination of both (magnetic and pressure forces) leads to the inward acceleration of the fluid. The role of the radial Lorentz term for driving inflows in the near surface layers becomes more important when we extract the CEFcarrying filaments only (dashed lines in Figure 4.6).

Similarly to the situation at the sources of the NEF, at the sources of the CEF the upflowing gas experiences a transition from more vertical into horizontal field due to a bending of the field occurring at the outer and middle penumbra (see e.g. the field inclination along the CEF filament shown in Figure 4.3). Thus, at the sources of the CEF the upflowing gas is partly deflected inwards by the magnetic field (note that part of the energy extracted by the magnetic field in the vertical direction is transferred into the inward acceleration of the gas). However, unlike the outflows, the inflows are primarily driven by radial pressure forces making the CEF a strong candidate for a siphon flow, which is further supported by our analysis in Section 4.5.

\subsubsection{Temporal evolution of the CEF}

Figure 4.7 follows the evolution in time of a portion of the penumbra with CEF. We focus on a single CEF patch only, since the time evolution of the CEF in the different parts of the penumbra is rather independent. But the general results described below are true for all the CEF patches in the simulated penumbra.

The figure shows in panels (a) the radial mass fluxes at three stages of the evolution of the CEF in the selected penumbral sector at $z=-226 \mathrm{~km}$, the negative values indicate mass flowing radially inwards. Panels (b), (c) and (d) in Figure 4.7 show that the source regions of the CEF at $z=-226 \mathrm{~km}$ that have positive values of $L_{r}^{-}$are all characterized by 


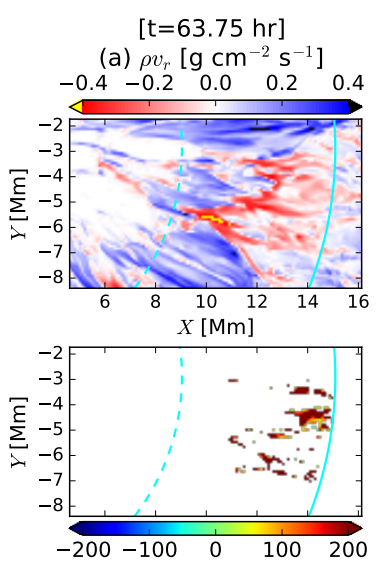

(b) $L_{r}^{-}\left[\mathrm{erg} \mathrm{cm} \mathrm{cm}^{-3} \mathrm{~s}^{-1}\right.$ ]

[t=65.25 hr]

(a) $\rho v_{r}\left[\mathrm{~g} \mathrm{~cm}^{-2} \mathrm{~s}^{-1}\right]$
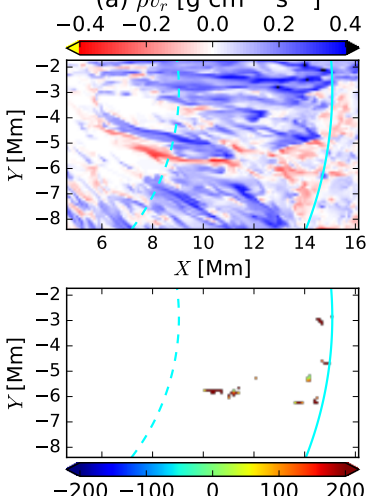

(b) $L_{r}^{-}\left[\mathrm{erg} \mathrm{cm}^{-3} \mathrm{~s}^{-1}\right]$

[t=68.5 hr]

(a) $\rho v_{r}\left[\mathrm{~g} \mathrm{~cm}^{-2} \mathrm{~s}^{-1}\right]$
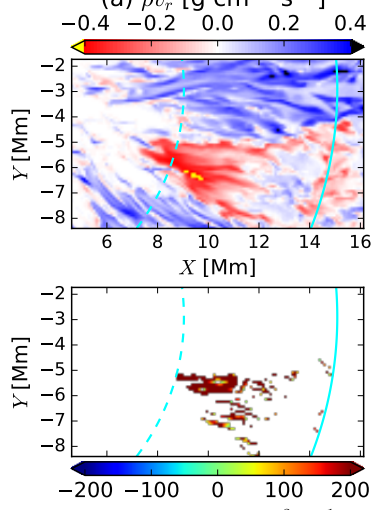

(b) $L_{r}^{-}$[erg cm $\mathrm{cm}^{-3} \mathrm{~s}^{-1}$ ]

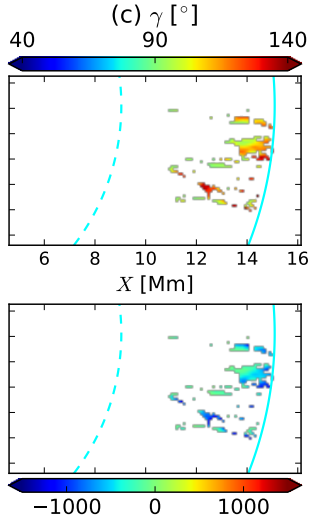

(d) $B_{z}[\mathrm{G}]$

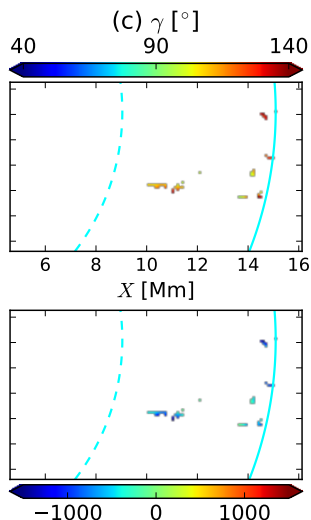

(d) $B_{z}[\mathrm{G}]$
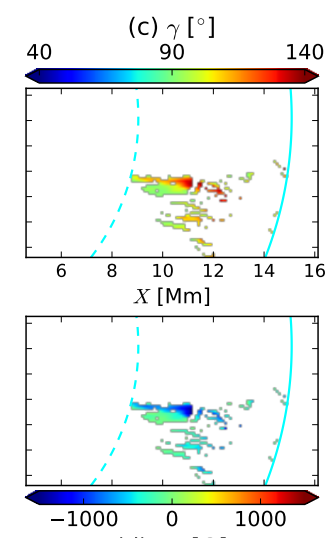

(d) $B_{z}[\mathrm{G}]$ (e) Radial terms
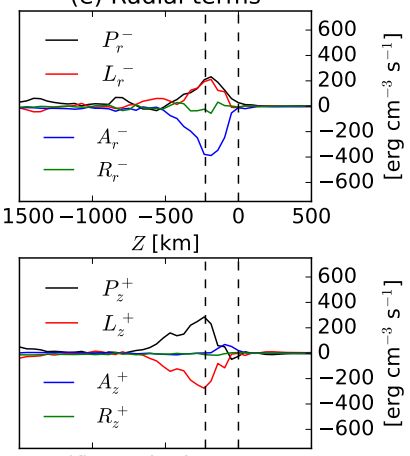

(f) Vertical terms

(e) Radial terms
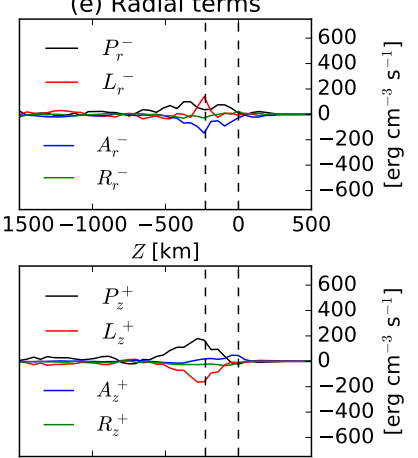

(f) Vertical terms

(e) Radial terms
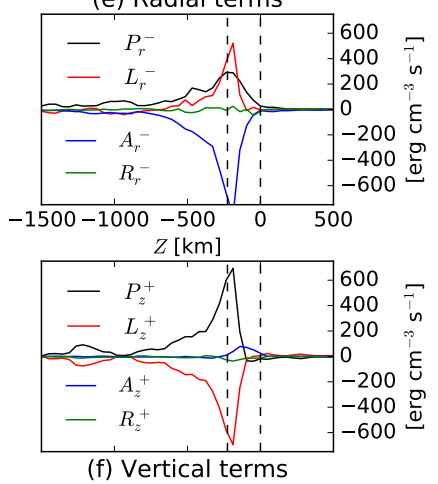

(f) Vertical terms

Figure 4.7: Variability of the CEF in a portion of the penumbra at three selected timesteps, from top to bottom, $t=63.75,65.25$ and 68.5 hours. For each time step six panels are plotted. Panel (a) displays the radial mass flux, $\rho v_{r}$, at $z=-226 \mathrm{~km}$. Panels (b), (c) and (d) show the quantities $L_{r}^{-}$, field inclination $\gamma$, and vertical field component $B_{z}$, respectively, in the regions responsible for driving the CEF at $z=-226 \mathrm{~km}$, where the term $L_{r}^{-}$has positive values. In panels (a)-(d) cyan curves were placed at $r=9 \mathrm{Mm}$ (dashed) and $r=15 \mathrm{Mm}$. Panels (e) and (f) depict the height profiles of the radial and the vertical energy conversion terms, respectively. The energy conversion terms have been averaged over the sources of the CEF at each height and time-step, using the same mask as in the dashed plots of Figure 4.6. The vertical dashed lines in panels (e) and (f) are placed at $z=0 \mathrm{~km}$ (average height of the $\tau=1$ level in the quiet sun) and at $z=-226 \mathrm{~km}$ (average height of the $\tau=1$ level in the penumbra). 

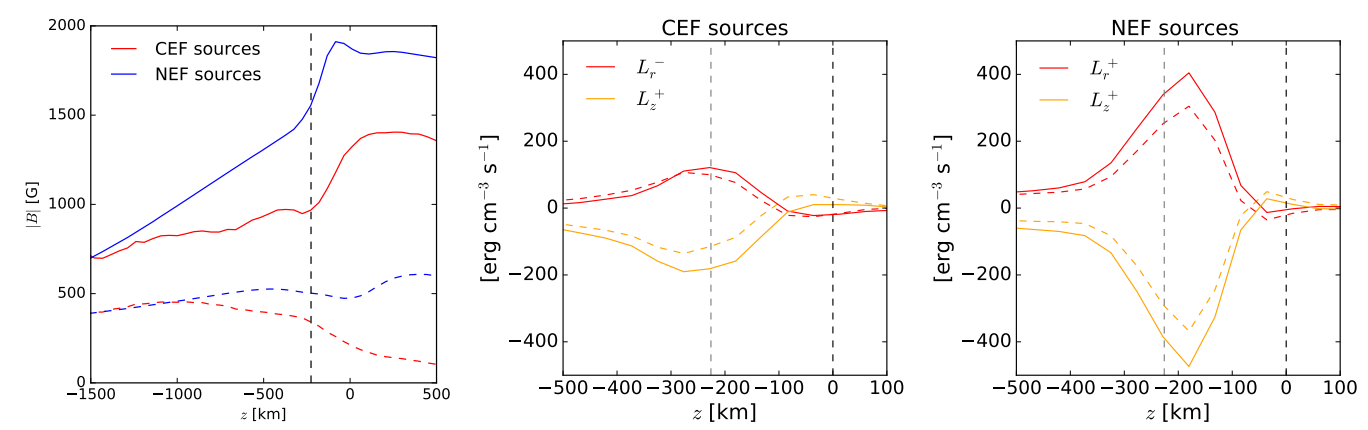

Figure 4.8: Left panel: Temporal average of the magnetic field vertical profiles in regions responsible for driving the CEF (red) and the NEF (blue). The magnetic field is separated according to its vertical (dashed) and radial (solid) components. The vertical dashed line is placed at $z=-226 \mathrm{~km}$. Middle panel: Energy conversion by radial (red) and vertical (orange) Lorentz force at the sources of the CEF. Solid lines are a close up view from the corresponding dashed lines in Figure 4.6. The dashed lines show the simplified expression: $L_{r}=v_{r} \frac{1}{4 \pi} B_{z} \frac{\partial B_{r}}{\partial z}$ (red) and $L_{z}=-v_{z} \frac{1}{4 \pi} B_{r} \frac{\partial B_{r}}{\partial z}$ (orange). Right panel: Same as in the middle panel for the sources of the NEF.
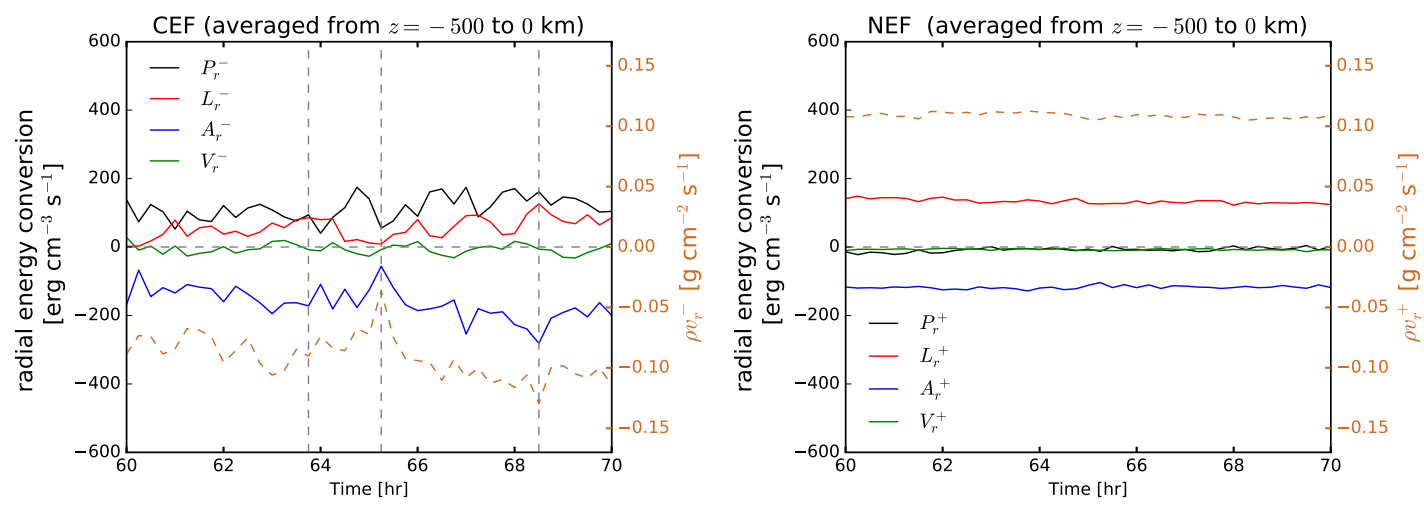

Figure 4.9: Temporal evolution of the radial forces (solid) and mass fluxes (dashed) at the sources of the CEF within the portion of penumbra shown in Figure 4.7 (left plots). At each time, the plots show the average value of each quantity in the near-surface layers (between $z=-500$ and $z=0 \mathrm{~km}$ ) from the middle to the outer penumbra. For comparison, we show the same average quantities for the sources of the NEF (right plots) averaged over the inner to the middle penumbra. Vertical dashed lines on the left panel indicate the three selected stages of the CEF that are shown in Figure 4.7. 
negative values of $B_{z}$, i.e. downward pointing fields $\left(\gamma>90^{\circ}\right)$ regardless of whether they are located in the middle or in the outer penumbra. In those regions, the field inclination increases radially inwards. Panels (e) show the radial energy conversion associated with the sources of the CEF in the different time-steps. The contribution of both, $L_{r}^{-}$and $P_{r}^{-}$to the inward acceleration of the fluid vary over time, being the increase of the overall radial energy conversion associated with an increase of the radial mass flux in the selected CEF patch. At all three stages of the CEF shown in Figure 4.7, the vertical pressure force shown in panels (f) presents a steepening close to $z=-500 \mathrm{~km}$ and it peaks near the average $\tau=1$ level of the penumbra $(z=-226 \mathrm{~km})$, keeping a close balance with work done against the Lorentz force.

In order to gain insight into the typical underlying magnetic structure of the regions responsible for driving the CEF, we show in Figure 4.8 (left panel) the average vertical and radial magnetic field as a function of height in regions that are sources of CEF (red) and in regions that are sources of NEF (blue). For the CEF sources, the vertical field component (dashed red line) remains constant at about $400 \mathrm{G}$ below $z=-226 \mathrm{~km}$ and decreases to $\sim 100 \mathrm{G}$ in higher layers. The radial field component (solid red line) increases monotonically from about $700 \mathrm{G}$ to $\sim 1000 \mathrm{G}$ below $z=-226 \mathrm{~km}$. At $z \sim-226 \mathrm{~km}$, the slope of $\left|B_{r}\right|$ suffers a large steepening and the radial field component increases to almost $1400 \mathrm{G}$ towards $z=0$ and remains nearly constant at higher layers. The steep increase of $\left|B_{r}\right|$ near $\tau=1$ combined with the reduction of $\left|B_{z}\right|$ results in a strong increase of the radial Lorentz force. As shown in the middle panel of Figure 4.8, there is a good agreement between the Lorentz terms as defined in equation 4.3 (solid lines) and the following approximations:

$$
\begin{aligned}
L_{z} & \approx-v_{z} \frac{1}{4 \pi} B_{r} \frac{\partial B_{r}}{\partial z} \\
L_{r} & \approx v_{r} \frac{1}{4 \pi} B_{z} \frac{\partial B_{r}}{\partial z}
\end{aligned}
$$

which are indicated by the dashed lines. Similar to what happens at the NEF sources (right panel), at the CEF sources the energy extracted in the vertical direction by the Lorentz force, $L_{z}$, is in approximate balance with the radial Lorentz force, $L_{r}$. Then, for both, NEF and CEF sources, the following is valid:

$$
v_{z} \frac{1}{4 \pi} B_{r} \frac{\partial B_{r}}{\partial z} \approx v_{r} \frac{1}{4 \pi} B_{z} \frac{\partial B_{r}}{\partial z}
$$

which leads to the relation $v_{z} B_{r} \approx v_{r} B_{z}$. This relation implies a deflection of the vertical pressure forces in upflows by the Lorentz force to allow for the strong acceleration in the radial direction and it is in agreement with the findings of Rempel (2011) for the driving mechanism of the NEF. Here, we show that this relation is also valid for the CEF, whose vertical flows occur in regions where the vertical field component is negative and its magnitude decreasing with height, while the upflowing gas encounters an increased radial field component when it reaches the surface. This results in a radial driving by the Lorentz force that favors inflows and accelerates the gas inwards with the help of radial pressure forces, which in this case are larger than the Lorentz force.

Similarly, at the source regions of the NEF, the average vertical field component (dashed blue line in left panel of Figure 4.8) remains around $\sim 400 \mathrm{G}$ whilst the radial field component (solid blue line) increases steadily from about $700 \mathrm{G}$ to $\sim 1500 \mathrm{G}$ 
just below $z=-226 \mathrm{~km}$. Just as at the CEF sources, the slope of $\left|B_{r}\right|$ suffers a strong steepening close to $z \sim-226 \mathrm{~km}$ so that $\left|B_{r}\right|$ increases up to $1900 \mathrm{G}$ towards $z=0$ and drops again in higher layers to about $1800 \mathrm{G}$. Note that the average increase of $\left|B_{r}\right|$ near $\tau=1$ is substantially larger for the NEF sources than for the CEF sources because they occur within different parts of the penumbra. This causes the contribution of the radial Lorentz term to be larger at the sources of the NEF than at the sources of the CEF.

Unlike the NEF, the CEF is driven at all times by the contribution of two different radial forces: $P_{r}^{-}$and $L_{r}^{-}$. Figure 4.9 shows the temporal evolution of the average radial mass flux in the near-surface layers (from $z=-500$ to $0 \mathrm{~km}$ ) at the sources of the CEF in the selected penumbral portion (left plots) and, for comparison, at the sources of the NEF (right plots). The plotted time interval covers the lifetime of the CEF (i.e. the time from 60 to $70 \mathrm{hr}$ ). The solid lines in Figure 4.9 show the average radial energy conversion terms in the near-surface layers at each time-step. The temporal evolution of the CEF shows that the negative radial mass flux (dashed line) is strongly variable compared to the $\mathrm{NEF}$ case, and that it is proportional to the radial acceleration, $A_{r}^{-}$. This means that the sum of the two radial forces, magnetic and pressure forces (note that $A_{r}^{-} \approx-\left(L_{r}^{-}+P_{r}^{-}\right)$), is responsible for modulating the inward mass flux of the CEF in the near surface layers.

Unlike the CEF, the positive radial mass flux of the NEF as well as the radial energy balance have a more steady behavior during the 10 hours of the analyzed interval, exhibiting the quasi-stationarity and robustness of the NEF feature when the penumbra is well-developed. The radial Lorentz terms keep in balance with the radial acceleration forces during the 10 hours here analyzed and are, consistently, the responsible forces driving the NEF in the radial direction.

\subsection{Induction equation}

Given that the horizontal (radial) component of the magnetic field in the penumbra plays a determinant role on the driving of penumbral flows, and in order to study how the radial field is maintained in the penumbra, we evaluate the different terms in the induction equation:

$$
\frac{\partial \vec{B}}{\partial t}=\underbrace{-(\vec{v} \cdot \nabla) \vec{B}}_{\text {Advection }}+\underbrace{(\vec{B} \cdot \nabla) \vec{v}}_{\text {Stretching }} \underbrace{-\vec{B}(\nabla \cdot \vec{v})}_{\text {Divergence }} .
$$

Figure 4.10 shows the contribution of each term in equation 4.9 for the maintenance of the radial field (plots on the left) and of the vertical field component (plots on the right) at the sources of the NEF (upper plots) and at the sources of the CEF (bottom plots).

For the radial field component the dominant source is the stretching term in both cases, inflows and outflows (Figures 4.10a and 4.10c, respectively), and the major contribution to this term comes from the vertical shear profile of the NEF, $B_{y} \frac{\partial v_{r}}{\partial y}>0$ (red dashed line in Figure 4.10a; recall that the radial flow velocity increases with height up to the surface and $B_{y}$ is positive at the sources of the NEF), although the term $B_{r} \frac{\partial v_{r}}{\partial r}>0$ also contributes; whereas for the inflows, the dominant term for the radial stretching is $B_{r} \frac{\partial v_{r}}{\partial r}>0$ (red dotted line in Figure 4.10c). The latter implies that the inflows are, to a larger extent, field-aligned flows which display a strong inward acceleration (the negative radial flow 
velocities decrease with the radial distance and $B_{r}$ is always positive in the penumbra). In this way, the inflowing gas is able to transfer kinetic energy into magnetic energy, and by that to maintain the radial field component.

In contrast, the advection term has a negative contribution for the maintenance of the radial field in both outflows and inflows. At the sources of outflows, this is mainly due to the upward increase of the radial field, $-v_{y} \frac{\partial B_{r}}{\partial y}<0$ (black dashed line in Figure 4.10a; recall that $v_{y}$ is positive at the sources of both outflows and inflows); whereas at the inflow sources, the dominant negative contribution to the advection term comes from the outward decrease of the radial field at the outer penumbra, $-v_{r} \frac{\partial B_{r}}{\partial r}<0$ (black dotted line in Figure $4.10 \mathrm{c}$; recall that $v_{r}<0$ for inflows and that the radial field increases outwards from the inner penumbra but decreases towards the outer penumbra, where the field bends back to the surface and becomes more vertical). Likewise, the diverging convective motions tend to reduce the radial field in both cases (blue lines).

For the vertical field component, advection is the primary mechanism that maintains the field in the outflows case. The positive sign in this case comes from the (on average) outward decrease of the vertical field, $-v_{r} \frac{\partial B_{y}}{\partial r}>0$ (black dashed line in Figure 4.10b), due to the radial bending of the field in the inner penumbra. For the inflows, the advection term contribution to the maintenance of the vertical field component is negative mainly due to the fact that the vertical field component becomes more negative with radial distance at the CEF sources, and therefore $-v_{r} \frac{\partial B_{y}}{\partial r}<0$ (black dashed line in Figure 4.10d).

The role of the contribution from vertical field stretching is also opposite for inflows and outflows. For the outflows, the dominant negative contribution to the vertical stretching term is due to $B_{y} \frac{\partial v_{y}}{\partial y}<0$ (red dashed line in Figure $4.10 \mathrm{~b}$ ), which has a minimum close to the surface due to the deceleration of the upflows (see Figure 4.5). In contrast, for the inflows, the vertical field is maintained by vertical stretching mainly due to the term $B_{r} \frac{\partial v_{y}}{\partial r}>0$ (red dotted line in Figure 4.10d). Finally, in both outflows and inflows, the remainder is offset by the negative and the positive contribution of the divergence term respectively (blue lines); which in the case of the inflow sources is positive due to the generally negative sign of the vertical field component at those places.

For both radial and vertical magnetic field components the contributions from stretching, advection and divergence are mostly in balance, implying that the numerical magnetic diffusivity (green lines) does not play a significant role on the magnetic structure of the penumbra in these simulations.

Figure 4.11 displays the contributions of each term in equation 4.9 horizontally averaged over the sinks of the NEF (left plot) and over the sinks of the CEF (right plot). Given that the vertical field component is noticeably enhanced at the supersonic sinks of both, NEF (mainly outer penumbra) and CEF (mainly inner penumbra), we explore the mechanisms that can lead to the amplification of the magnetic field strength at those places (see e.g. Figure 4.3). At the sinks of the NEF, there is a clear positive contribution from the vertical advective term, which increases near the heights of the supersonic downflows of the NEF (cf. white contours in $v_{z}$ panel of Figure 4.3). Therefore, a possible mechanism responsible for the local intensification of the magnetic field at the supersonic sinks of the NEF is the downdraft of magnetic flux concentrations by the supersonic downflows in the outer penumbra. In contrast, advection plays an opposite role at the sinks of the CEF, where the dominant positive contribution to the vertical field is given by the divergence 


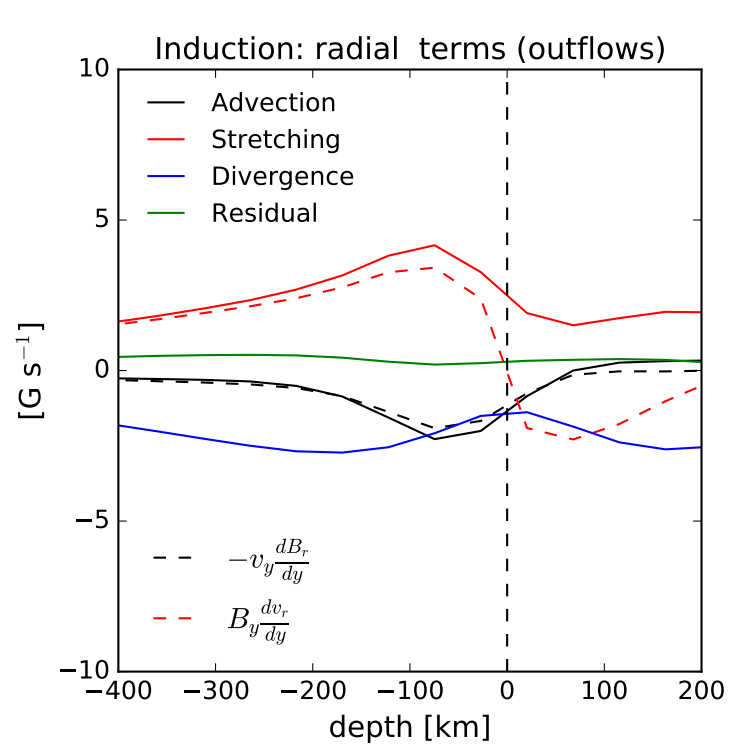

(a)

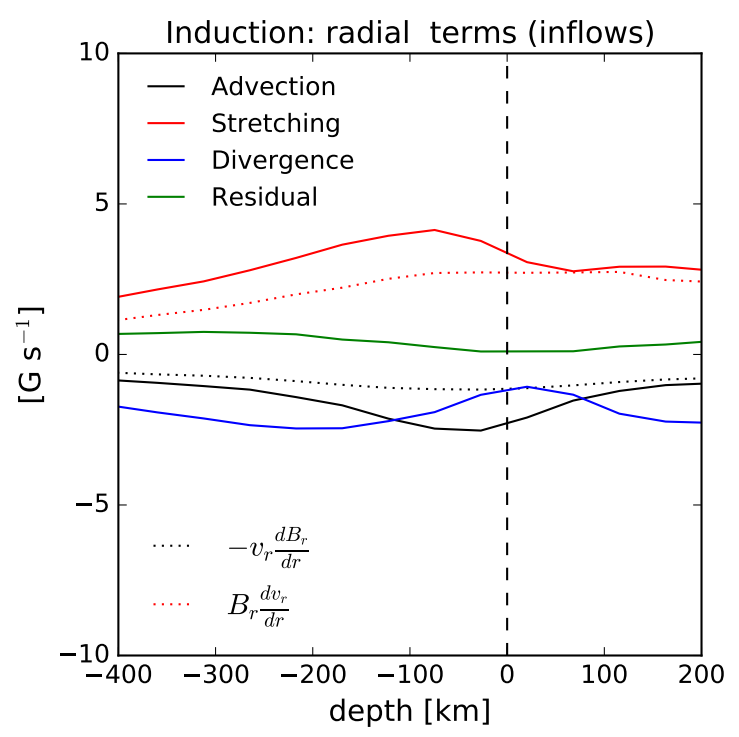

(c)

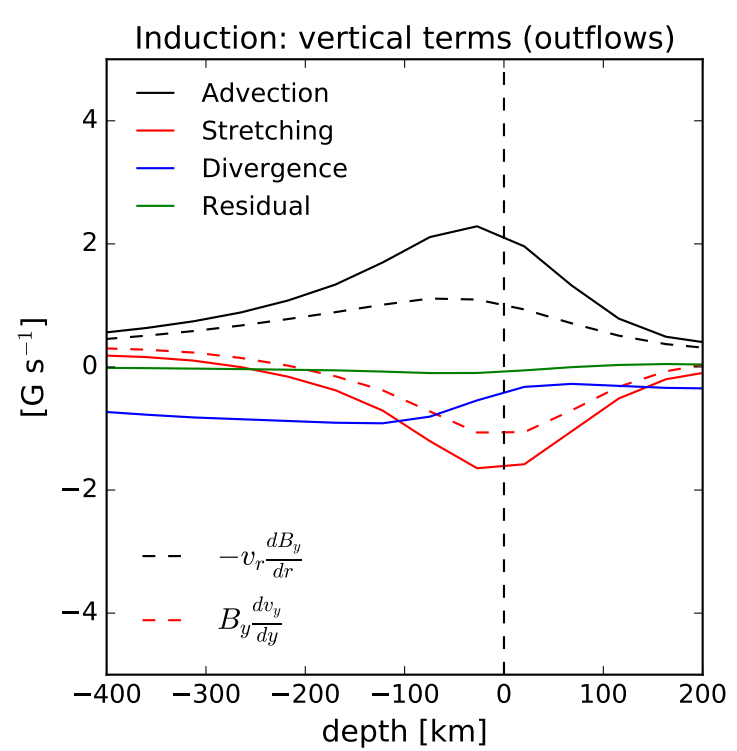

(b)

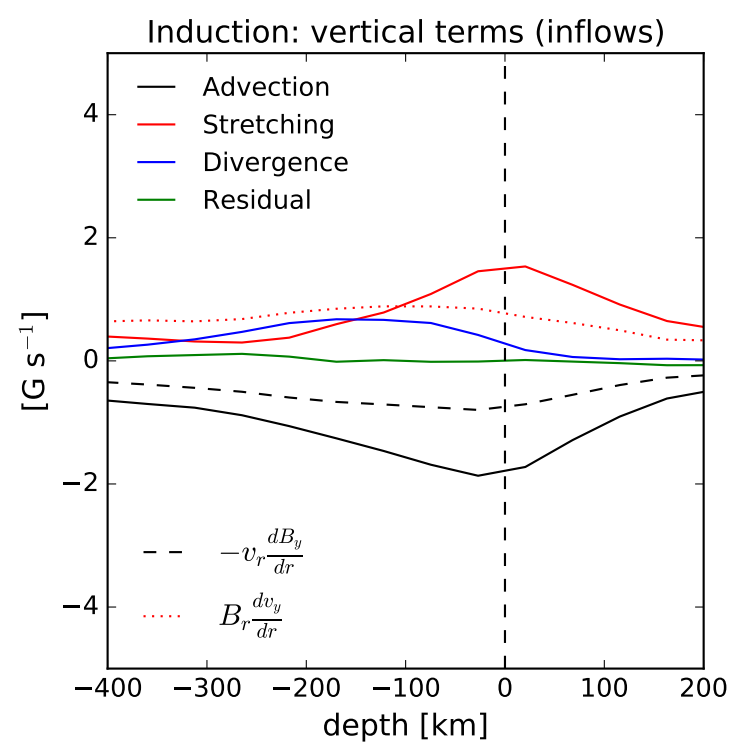

(d)

Figure 4.10: Average contributions in induction equation from advection (solid black), field-line stretching (solid red), flow divergence (solid blue) and residual (solid green) at the sources of the NEF (upper plots) and at the sources of the CEF (lower plots). Panels on the left display the terms for the radial field component and panels on the right for the vertical field component. Here, dashed and dotted lines represent different terms that contribute to the advection terms (black) and to the stretching terms (red) and whose general expressions are indicated in the lower part of each panel. The vertical dashed line is placed at $z=0 \mathrm{~km}$. 

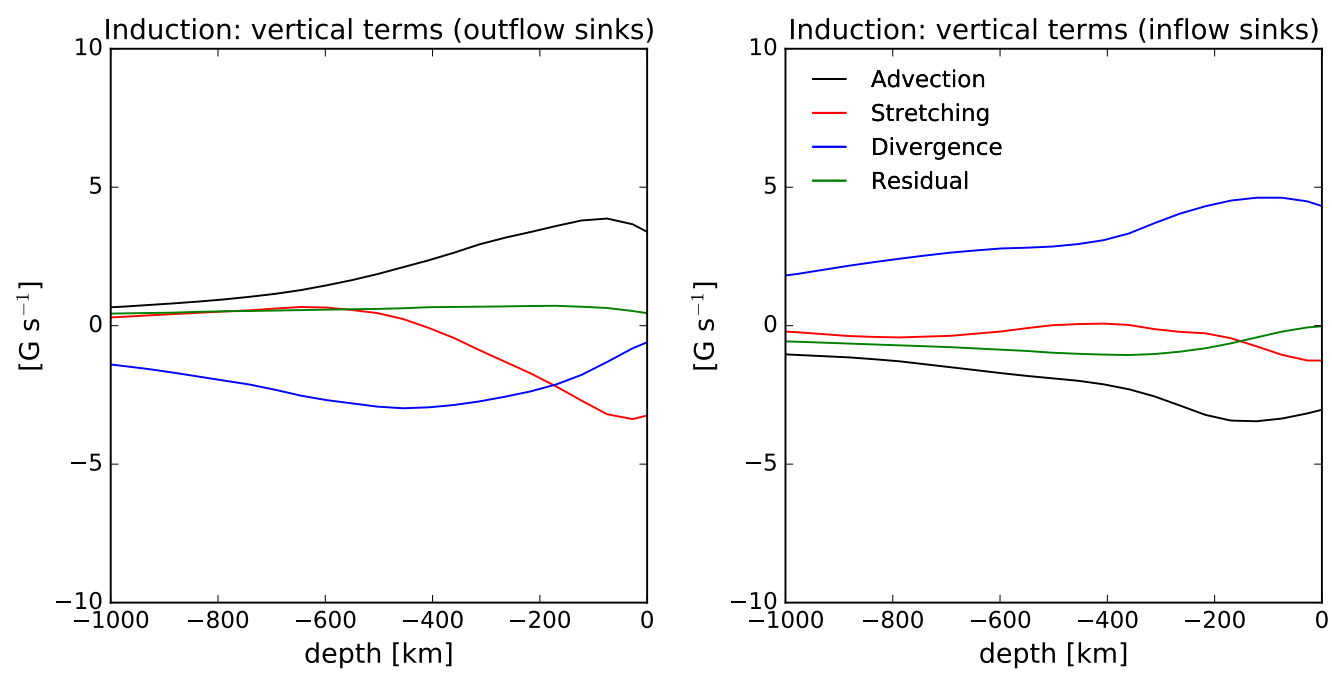

Figure 4.11: Average contributions to the induction equation from advection (black), field-line stretching (red), flow divergence (blue) and residual (green) to the vertical field component at the sinks of the NEF (left) and at the sinks of the CEF (right).

term (i.e. $\nabla \cdot \vec{v}<0$ ) which means that the CEF sinks are convergent downflows and implies compression and amplification of the magnetic field.

\subsection{Field-line connectivity}

We have shown in the previous analysis that the CEF and the NEF are both driven within a thin boundary layer close to the $\tau=1$ level, and that the velocity vector is mostly radial. Moreover, we found that strong radial pressure gradients exist everywhere within the thin boundary layer in which the CEFs are driven. In contrast, while the radial pressure forces have an on average opposed contribution to the driving of the NEF in the source regions, positive radial pressure forces dominate the average in the outer penumbra where the NEF sinks (Figure 4.4). Although the driving of the flows depends principally on the conditions in their respective upflow cells, identifying the magnetic connectivity of the field lines is an important aspect to describe individual flow channels (penumbral filaments) as flux tubes, and therefore is also necessary to validate whether the flows are or are not driven by a siphon flow mechanism. Nonetheless, such a representation involves some limitations when compared with models based on the thin flux tube approximation (e.g., Meyer and Schmidt 1968b, Thomas and Montesinos 1993, Montesinos and Thomas 1997, Schlichenmaier et al. 1998a) which assume well-defined footpoints of the flux tubes on horizontal cuts made at given heights below the surface. In particular, the NEF-carrying filaments in this simulation (and in that by Rempel 2011) display elongated upflow cells which constrain their representation under the thin flux tube picture.

To study the magnetic field-line connectivity as well as the associated field-aligned velocity component and pressure perturbation along the different types of filaments in the penumbra (NEF carrying and CEF carrying filaments), we use the VAPOR software package developed at NCAR (Clyne and Rast 2005, Clyne et al. 2007, http://www.vapor.ucar.edu).

In Figure 4.12, we have selected field lines in a cross-section perpendicular to fila- 

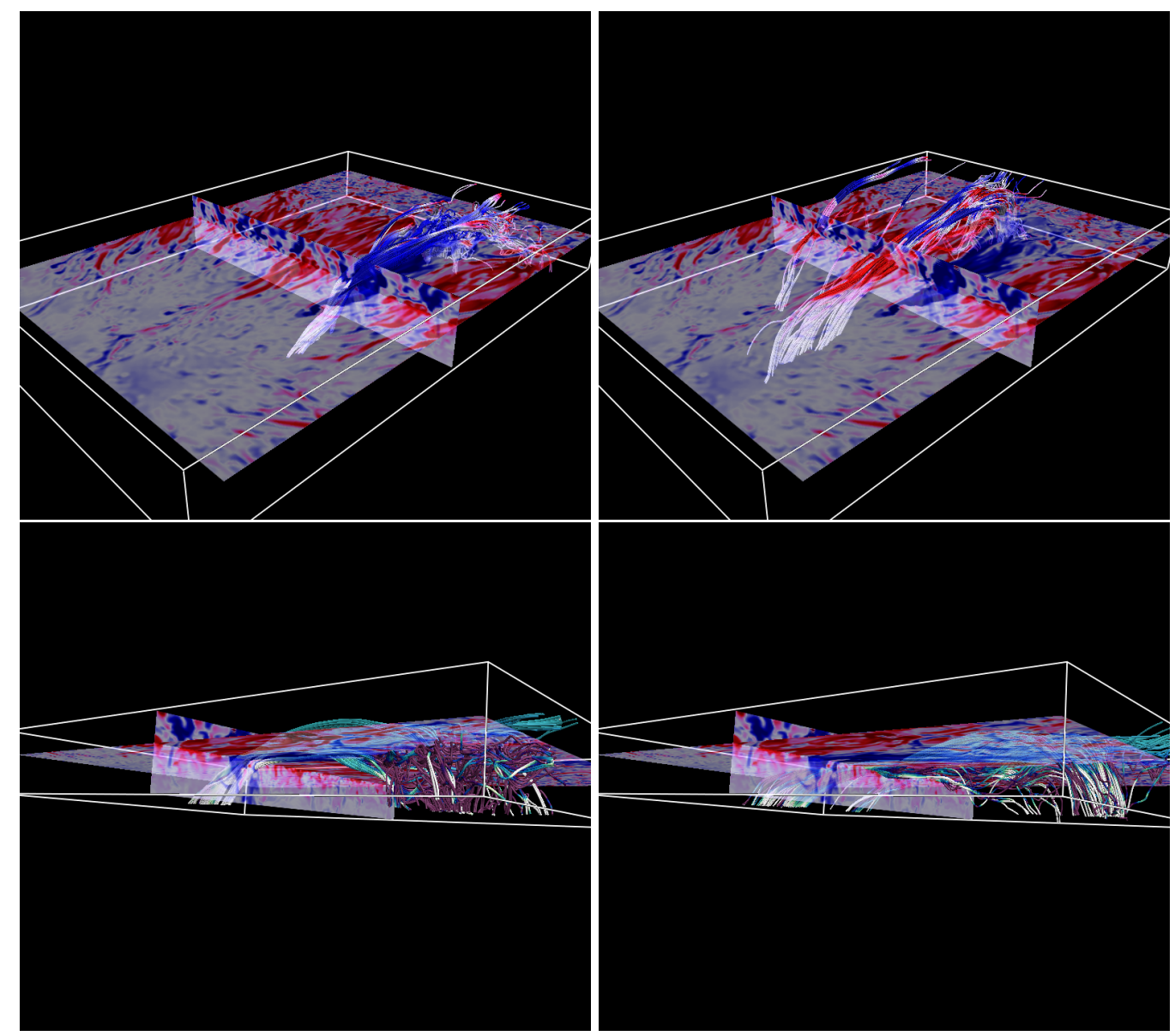

Figure 4.12: Field-line connectivity and associated velocity and pressure perturbations in the simulated penumbra: CEF filaments (left figures) and NEF filaments (right figures). In all figures the semi-transparent horizontal and vertical planes show the field-aligned velocity component from $\pm 10 \mathrm{~km} \mathrm{~s}^{-1}$ near the average $\tau=1$ level, outflows are red and inflows are blue. The vertical plane is placed close to the inner penumbral boundary and is used to select field lines by their flow velocity. In the upper figures, the color of the field lines shows the flow velocity along the field. In the bottom figures, the color of the field lines indicates the pressure perturbation along the field lines from $\pm 10^{5} \mathrm{dyne} / \mathrm{cm}^{2}$, where white is low and purple is high pressure. The upper and lower panels refer to different view angles. The plots correspond to the snapshot at $t=65 \mathrm{hr}$. 
ments based on regions with strong horizontal flows. The selected field lines were colored according to their field-aligned velocity in the upper images and according to the gas pressure perturbation along the field lines in the bottom images (we compute the pressure perturbation after subtracting the hydrostatic mean, since stratification dominates). The images on the left show field lines carrying a CEF from two different view angles and the images on the right show field lines carrying a NEF. In the case of the CEF filaments, most of the field lines span from the outer to the inner penumbra with a consistent inflow along them (top left panel) and they have a very systematic pressure perturbation (bottom left panel) with the gas flowing from high pressure regions (purple) to low pressure regions (white), consistent with a siphon flow.

In the case of the NEF, the velocity along the field lines is less consistent and some field lines have even an inverse flow in their upper portion (top right panel), similarly to the findings of Rempel (2011). In particular, in the inner penumbra, field lines that host the fastest Evershed flows still connect to the top boundary, but forming small dips inbetween their extremes. This indicates that the NEF is to a lesser degree a flow along the field and it implies continuous reconnection and change of field line connectivity as the mass moves outward in the penumbra. Moreover, it implies that the physical conditions in the outer footpoint have a minor impact on the driving of the NEF. Looking at the pressure perturbation (bottom right), we find a mix of high and low pressure at both footpoints. There could still be siphon flows along some field lines (mostly in the outer penumbra), but they do not provide a consistent explanation for the Evershed flow in the bulk of the penumbra.

\subsection{Discussion and conclusion}

We presented a detailed analysis of the properties of penumbral fine structure associated with the counter Evershed flows (CEF) and the normal Evershed outflows (NEF) near $\tau=1$ in the penumbra of the recent numerical sunspot simulation by Rempel (2015). Our investigation mainly focuses on the physical driving mechanisms of the NEF and the CEF in the near surface layers of this simulation.

The main difference found between the penumbral filaments carrying the NEF and the ones carrying the CEF is the location within the penumbra and the radial extent of the upflow cells. Both aspects being crucial for the driving of each flow given that their driving mechanisms depend primarily on the conditions present in their respective upflow cells.

The energy conversion analysis at the sources of both, outflows and inflows, suggests that the CEF and the NEF are both driven within a thin boundary layer close to the $\tau=1$ level, and no substantial driving forces exist well above $\tau=1$. In both cases, the loss of buoyancy due to radiative cooling is seen as a change of sign of the work by pressure in the vertical direction close to $z=0$ (Figure 4.6). However, while this provides only a small contribution to the deceleration of the vertical flow in the case of the NEF, i.e. most of the deceleration happens deeper down where the pressure driving is still positive and the dominant offset is given by the vertical Lorentz force, the deceleration of upflows by loss of buoyancy seems to be more prominent for the CEF near $z=0$. Nonetheless, deceleration of upflows in CEFs near $\tau=1$ occurs mainly due to an opposed contribution 
from the vertical Lorentz force, similarly to the NEF case. Therefore, notable differences exist with respect to the underlying driving forces of both, NEF and CEF, compared to field-free convection where deceleration of vertical flows near the surface is mainly due to loss of buoyancy caused by the radiative cooling (see Rempel 2011, who analyzed in detail the driving of the NEF in a simulated sunspot penumbra and made a comparison between the NEF and the plage region surrounding the sunspot, and found that the vertical pressure/buoyancy breaking has very clear distinct signatures in granulation and in the penumbra).

For the driving of the NEF, we found similar results to those reported by Rempel (2011). There is an almost complete energy balance between vertical pressure and Lorentz forces in upflows, as well as between the Lorentz force and acceleration in the radial direction. We found no significant kinetic energy in the vertical component at the sources of the NEF but a strong acceleration in the radial one in the near-surface layers. This implies that the flow changes direction and gains at the same time significant amounts of kinetic energy in the radial direction. Since the Lorentz force does not do any net work but only changes the direction of the flow, we can say that the flow is deflected by the highly inclined magnetic field in the penumbra and the gas is accelerated in the radial direction as a result of mass flux conservation coupled with the low gas density at the surface. The most important feature driving outflows in the inner and middle penumbra is the increase of the radial field component close to $\tau=1$. These results strongly support the magnetoconvective driver scenario of the NEF, as proposed in Scharmer et al. (2008), where the $\mathrm{NEF}$ is described as the horizontal component of overturning convection in penumbrae.

It has also been proposed in some models that the NEF could be the result of stationary siphon flows driven by a gas pressure difference between the footpoints of the penumbral filaments (e.g. Meyer and Schmidt 1968b). Such models consider processes related to turbulent pumping near the outer penumbral boundary (Montesinos and Thomas 1997, Brummell et al. 2008) which make the field lines bend downwards, and additionally produce a field strength enhancement in the outer filament endpoint due to strong downdrafts (van Noort et al. 2013). Such a field enhancement would establish a gas pressure gradient between the filament endpoints, producing a siphon-like outflow along the field lines. We do observe a net field strengthening at the outer endpoints of the NEF-carrying filaments (see e.g. Figure 4.3), at the places where the NEF sinks in the outer penumbra. This enhanced field could explain the increase of the radial pressure gradient towards the outer penumbra seen in the bottom right plot in Figure 4.4. However, the radial pressure gradients do not play an important role in the inner and in the middle penumbra, where most of the driving of the NEF occurs. This can be attributed to the elongated aspect of the upflow cells, which produces a substantial reduction of the radial pressure gradient. The fact that the largest average outflow speeds in the penumbra $\left(>5 \mathrm{~km} \mathrm{~s}^{-1}\right)$ stand out towards the outer penumbra (see radial velocity profile of the outflows in Figure 4.4) might be in part due to a larger fill factor of outflows in the outer penumbra that dominates the average. According to our results, the overall picture is that of a NEF mainly driven by magneto-convection in the inner and middle penumbra, in agreement with the results of Rempel (2011).

Observational studies of sunspots have shown that the penumbral filaments carrying the NEF in penumbrae have well defined "footpoints" at $\tau=1$, see e.g. Tiwari et al. (2013). The filaments footpoints are seen to confine the upflows (sources) and the down- 
flows (sinks) associated to the Evershed flow in the penumbra, separately. This is an important difference given that in these simulations the upflow cell covers a large portion of the NEF filaments, meaning that almost the entire filaments behave as a "footpoint". However, the lack of information on a true geometrical height scale in the current observational analysis techniques makes the comparison of numerical models with the highestresolution observations very challenging, unless the simulations include the computation of the observational quantities using radiative transfer computations (forward computations).

Overall, our analysis of the NEF essentially reinforces conclusions of Scharmer et al. (2008), Rempel et al. (2009a) and Rempel (2011) stating that the penumbra is dominated by anisotropic magnetoconvection and that the NEF can be understood as the convective flow component in the direction of the magnetic field, i.e., the overall underlying energy source is convective instability.

Unlike the NEF, the driving of the CEF occurs mainly in the middle and outer penumbra and the footpoints (sources and sinks) of the CEF-carrying filaments are generally well separated from each other. Furthermore, the upflow cells in the CEF-carrying filaments are less spread out compared to the NEF, i.e. less elongated in the radial direction, which enhances the role of the radial pressure gradients and allows for the existence of siphon-like inflows along the field lines that are connecting to the inner penumbra.

In addition to the radial pressure gradients, the CEF is also affected by the inclined field of the penumbra, in a similar way as the NEF is, i.e. that the radial Lorentz force plays an important role in the driving of the CEFs. The combination of both radial forces, results in fast inflows ( $>5 \mathrm{~km} \mathrm{~s}^{-1}$ on average) near the $\tau=1$ level. We found that, irrespective of whether the sources of the CEF are located in the middle or in the outer penumbra, the upflowing gas suffers a reduction of the vertical magnetic field combined with an enhancement of the radial field component. This leads to a positive contribution of the radial Lorentz force for the driving of the CEF.

Nonetheless, the energy conversion associated to the radial Lorentz force is on average smaller in the CEF sources than in the NEF sources given that the magnetic field is on average weaker in the outer penumbra than in the inner penumbra. This would at first sight imply a smaller radial acceleration of the CEF compared to the NEF. However, it is generally the sum of both, the radial pressure force and the radial Lorentz force that determines the radial acceleration of the CEF. Consequently, the inward acceleration of the fluid can eventually become even larger than that of the NEF. This occurs at several stages of the evolution of the CEF in the analyzed simulation. However, during the 10 hours analyzed in these simulations, the CEF shows up as a highly variable and unstable flow, while the NEF appears as a quasi-stationary and more robust feature in the penumbra.

Both, the CEF and the NEF are strongly magnetized and are driven within a thin boundary layer close to $\tau=1$. There are also other, smaller scale flows (outflows and inflows) in the penumbra that are unrelated to the filamentary penumbra. In particular, the additional inflows are mostly driven by pressure gradients in regions with an (on average) opposed contribution of the radial Lorentz term, the latter favoring outflows. This could explain why these inflows are of much smaller scales than the CEF.

According to observational studies, CEFs can occur under different physical circumstances. On the one hand, those CEFs that have been observed during the early stages of penumbra formation (e.g., Schlichenmaier et al. 2012, Romano et al. 2014, Murabito 
et al. 2016) usually appear as elongated patches at the outer edge of the proto-spot, and are unrelated to any filamentary structure since they are observed when the latter is not yet developed. Furthermore, the newly formed penumbral filaments host a NEF soon after their formation. Therefore, those inflows observed around forming sunspots may involve different physical driving mechanisms since they are essentially different to the CEFs studied in this simulation. A possible scenario is that they are driven only by gas pressure gradients which are caused by the increase of the magnetic field strength in the proto-spot.

On the other hand, the CEFs that have been observed (albeit more rarely) in welldeveloped penumbrae (Kleint and Sainz Dalda 2013, Louis et al. 2014, Siu-Tapia et al. 2017) share important similarities with the CEFs in the present simulations: they were observed either along singular penumbral filaments or, as in Siu-Tapia et al. (2017), along an array of penumbral filaments covering a sizable part of the penumbra in a mature spot, but with most of the penumbra still displaying the NEF as in our present simulation. This occurs in all the three above-mentioned reported observations of CEFs in well-developed penumbrae. However, due to the lack of knowledge of the true geometrical height scale in the observations and the inability to measure vertical gradients below the photosphere, none of these works could determine the dominant forces driving the flows.

In particular, Siu-Tapia et al. (2017) reported the observation of a prominent CEF with lifetimes of $\sim 2$ days at photospheric heights. Similar to the simulations analyzed here, the CEF in those observations showed an associated filamentary structure, with the sources of the CEF (hot upflows in vertical field regions) identified in the outer penumbra and the sinks (cooler downflows in strong vertical field regions) at the inner penumbral boundary. Furthermore, we found that the general magnetic, thermal and velocity structure along the central axes of the CEF-carrying filaments at $\tau=1$ are remarkably similar to those reported in that work. Of particular interest is their finding of an enhanced temperature at the sinks of the CEF with respect to the surrounding environment and a very large field strengthening associated to supersonic downflow speeds. We also see these two aspects at the sinks of the CEF in the simulations. On the one hand, we found that deceleration takes place in the form of shocks at the sinks of both, CEF- and NEF-carrying filaments. This can explain the local temperature rise found at their sinks.

On the other hand, we found enhanced field strengths at the sinks of both, CEF and $\mathrm{NEF}$, reaching values up to $\sim 5 \mathrm{kG}$ and $\sim 2.5 \mathrm{kG}$ respectively. The very low densities of the downflowing gas produces the depression of the local $\tau=1$ level at the sinks of both, NEF and CEF. At the CEF sinks, the $\tau=1$ depression combined with the influence of the umbral field contributes to the observation of such strong fields at those places; while at the NEF sinks, the local enhancement of the field is the result of the $\tau=1$ depression combined with a net magnetic field intensification that is well-localized in height and might be produced by the supersonic downdrafts of magnetic flux at the outer penumbra, as proposed by van Noort et al. (2013). The latter is supported by the fact that, at the sink places of the NEF (mainly outer penumbra), vertical advection processes can feed and locally intensify the vertical field component at those places (Figure 4.11). By contrast, the strong magnetic fields found at the sinks of the CEF (combined with the above described responsible factors) can be the result of a field intensification due to the presence of converging downflows that compress and amplify the vertical field component in the inner penumbra. The occurrence of converging downflows at the inner 
footpoint of the penumbral filaments (CEF sinks) can be explained under the flux tube representation of the penumbral filaments if the gas flows through asymmetric flux tubes whose cross-sectional area decreases in the flow direction. This can occur in the CEF filaments because the magnetic field intensity increases inwards in the penumbra, and the magnetic flux along a flux tube must remain constant.

The CEF in these simulations persists up to $\sim 10$ hours, which is much shorter than the lifetimes reported by Siu-Tapia et al. (2017), although the feature studied by SiuTapia et al. (2017) was extraordinary compared with other observed CEF events (see e.g. Kleint and Sainz Dalda 2013, Louis et al. 2014). However, in both cases, the CEF has much shorter lifetimes than the NEF and can be thought of as transitory events relative to the latter. Furthermore, similar to what happens in these simulations, Siu-Tapia et al. (2017) reported that the penumbral sector harboring the CEF displays only a NEF after the CEF disappears. We have not studied the exact mechanism how the CEF is reversed to a NEF. However, a negative contribution of the radial Lorentz term combined with a strong reduction of the radial pressure gradient at the sources of the CEF would strongly favor the driving of outflows instead. A physical mechanism leading to the change of the magnetic field configuration and of the plasma conditions in the penumbra in such a way would likely involve magnetic reconnection.

Our analysis of the driving forces of the CEF in the simulated penumbra clearly shows that the CEF is a siphon flow driven by pressure gradients along the penumbral filaments. The inclined field in the penumbral filaments causes the radial Lorentz force to also play an important role in accelerating the gas inwards. Furthermore, besides the most prominent and persistent groups of CEF-carrying filaments during the analyzed temporal interval (e.g. those shown in Figure 4.2 and the portion analyzed in Figures 4.7 and 4.9), there are also a few other CEF-carrying filaments that live shorter and appear intermittently at different azimuths in the penumbra. We do not discard the possibility that CEFs may actually occur along penumbral filaments more regularly than observed but their short lifetimes prevents them being easily observed. According to our results, that would occur whenever a significant pressure gradient favoring CEFs is established in a penumbral filament, as in those cases in which the outer footpoint of the filament is not strengthened with respect to the inner footpoint (see, e.g., the CEF-carrying filament shown in Figure 4.3). Alternatively, CEFs may also appear as a consequence of ongoing magnetic flux emergence in the penumbra. This interpretation was presented by Chen et al. (2017). In their recent MHD simulation more than $60 \%$ of the simulated penumbra is dominated by CEFs that result from the mass drain into the umbra-penumbra boundary along the newly emerged field lines that coalesce and contribute to the horizontal field in the penumbra.

Nonetheless, there are very few reported observations of CEFs in sunspot well-developed penumbrae (Kleint and Sainz Dalda 2013, Louis et al. 2014, Siu-Tapia et al. 2017). This could also be due to a combination of their rare occurrence and their short lifetimes. However, more theoretical studies on this topic as well as high-temporal-cadence spectropolarimetric observations are necessary to investigate the true occurrence frequency and to learn more about the nature of CEFs in well developed sunspot penumbrae as well as their possible influence on the upper atmosphere.

Although the question of a IEF-CEF connection is compelling, the IEF-like feature that dominates the penumbral velocity profile near the $\log (\tau)=-4$ level (Figure 4.2) has not been analyzed here. IEF-like features appear to be a robust aspect in various sunspot 
MHD simulations (e.g., Rempel et al. 2009a, Rempel 2011, 2012a, 2014, 2015). However, this persistent feature in the simulations has not yet been analyzed given that the field lines hosting this flow frequently connect to the top boundary, which is numerically closed in most of the cases implying that these inflows are likely influenced by numerical diffusion or some leakage across the boundary. In those regions the Lorentz force is artificially limited to keep the Alfvén velocity low, which leads to energetic inconsistencies that make an energetic analysis tricky. To address this problem, new approaches in the scope of semi-relativistic MHD with an artificially reduced speed of light (this is typically called the "Boris correction", Boris 1970) are currently being explored (e.g., Rempel 2017), but this was not yet implemented in this simulation. 


\section{Unusually large penumbral field strengths}

\subsection{Introduction}

Sunspots are the largest concentrations of magnetic flux on the solar surface and, typically, the strongest magnetic fields are found in their umbral regions. The maximum field strength ever measured in a sunspot umbra was found by Livingston and Harvey (2006) to reach $6.1 \mathrm{kG}$. However, at present, an upper limit for the local magnetic field strengths in sunspots has not yet been established.

Lozitsky (1993) proposed the existence of sunspot-flare related 'superstrong' fields (above $5 \mathrm{kG}$ and even beyond $50 \mathrm{kG}$ ) whose presence could only be deduced from multiline profile observations since they might occur at small spatial scales, i.e. sub-telescopic scales and with low filling factors. The reality of Lozitsky's superstrong fields was never independently confirmed. Nonetheless, the mere possibility of such superstrong fields in sunspots leads to the question, what are the necessary physical conditions for their appearance, and more importantly, what are their effects on the current ideas about the physics of sunspots considered by the theoretical models? Certainly, as argued by van Noort et al. (2013), under the presence of superstrong magnetic fields the typically assumed approximation of hydrostatic equilibrium (in time-independent situations) the gravity and the gas pressure are likely not the dominant terms in the momentum equation. However, this is valid only if the field is not vertical. For vertical fields, hydrostatic equilibrium will be satisfied, as the field does not affect the stratification along the field lines.

The very large magnetic field strengths, $B \geq 5 \mathrm{kG}$, inferred by using the SPINOR 2D inversion code at all three height nodes $(\log (\tau)=-2.0,-0.8$ and 0$)$ in the inner penumbra of the main sunspot in AR 10930 (see Chapter 3 and Fig. 5.1) are among the largest field strengths ever observed in penumbral environments (see also van Noort et al. 2013). In particular, field strengths above $7 \mathrm{kG}$ (yellow markers in Fig. 5.1) are even larger than the largest umbral fields found by Livingston and Harvey (2006) of $~ 6.1 \mathrm{kG}$, who also found that only a very small fraction of sunspots (around the $0.2 \%$ in a 9 -decade record of $\sim 32000$ sunspots) have umbral fields stronger than $4 \mathrm{kG}$. The existence of field strengths in excess of $7 \mathrm{kG}$ possibly have an impact on the approximations made by the inversion code, which can lead to errors in the density stratifications if the non-vertical field components are significant, and therefore in the atmospheric stratifications of the physical parameters inferred by the code. Hence, the reliability of our inversion results in such peculiar pixels needs to be tested.

This chapter is aimed at discussing the reliability of those magnetic field values that 


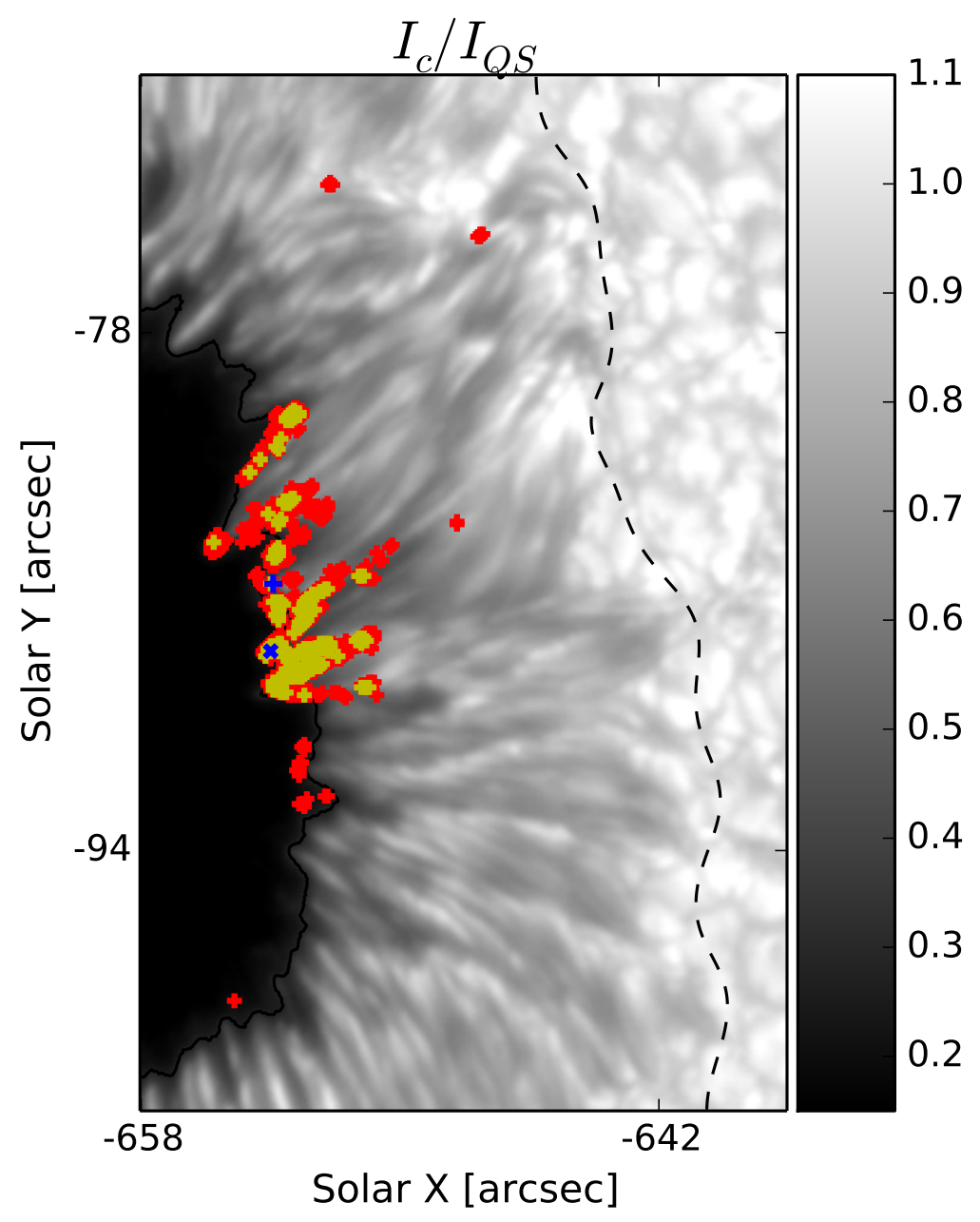

Figure 5.1: Continuum intensity map of the center-side penumbra of the main sunspot in AR 10930. Red markers indicate the location of 845 pixels where the SPINOR 2D inversions return $B \geq 5 \mathrm{kG}$ at $\log (\tau)=0$, of which 226 harbor fields larger than $7 \mathrm{kG}$ at $\log (\tau)=0$ (yellow markers). The blue markers highlight the location of two pixels with $B \geq 7 \mathrm{kG}$ ('+': pixel 1, 'x': pixel 2) selected to test the robustness of our inversion solutions (see text). 
5.2 Zeeman splitting and center-of-gravity methods

\begin{tabular}{c|ccc}
\hline method & pixel & $B[\mathrm{kG}]$ & $B[\mathrm{kG}]$ \\
$(6301.5 \AA)$ & $(6302.5 \AA)$ \\
\hline \multirow{2}{*}{ 1. ZEEMAN (Stokes $V)$} & 1 & 9.4 & 7.9 \\
& 2 & 9.4 & 7.2 \\
\hline \multirow{2}{*}{ 2. ZEEMAN (Stokes $I)$} & 1 & & 7.1 \\
& 2 & & 6.9 \\
\hline \multirow{2}{*}{ 3. COG } & 1 & 2.6 & 3.9 \\
& 2 & 2.7 & 3.6 \\
\hline
\end{tabular}

Table 5.1: Results of Zeeman and center-of-gravity (COG) methods.

are larger than $7 \mathrm{kG}$. Here we apply some classical diagnostic methods and various inversion techniques with different model atmospheres to the Stokes profiles from such large-field pixels (LFPs) in order to find out which method provides the most reliable results. We do not provide uncertainties and formal errors in the inverted physical parameters since the main errors depend largely on the chosen model; instead, we provide the reader with approximated values of the inverted parameters only.

\subsection{Zeeman splitting and center-of-gravity methods}

Most of the pixels where the SPINOR 2D inversions return field strengths larger than $7 \mathrm{kG}$ (hereafter LFPs, yellow markers in Fig. 5.1) are located at or close to the umbral/penumbral boundary of the penumbral region hosting a counter-Evershed flow (CEF), and contain very complex Stokes profiles. Figure 5.2 shows the observed Stokes profiles (black dashed curves) from two selected LFPs. The locations of the selected LFPs are marked with blue crosses in Figure 5.1 ('+': pixel 1, 'x': pixel 2). All Stokes parameters in these LFPs exhibit large asymmetries and the Stokes $V$ profiles display more than two lobes. Their best fits from SPINOR 2D (e.g., orange curves in Fig. 5.5) are not nearly as good as in most of the penumbral pixels (cf. Chapter 3). It is noticeable that the continuum intensity and the Stokes $V$ profiles are only imperfectly reproduced in both examples.

As a very simple alternative estimation of the field strength in the selected LFPs, we compute the magnetic field strength directly from the splitting of the two observed Fe I line profiles at $6301.5 \AA$ (line 1) and $6302.5 \AA$ (line 2), using the Zeeman splitting formula:

$$
B=\frac{\lambda^{+}-\lambda^{-}}{2.0} \frac{4 \pi m c}{e g_{L} \lambda_{0}^{2}}
$$

where $\lambda_{0}$ is the central wavelength of the line, $\lambda^{ \pm}$are the centroids of the right and left circular polarized line components ( $\sigma$-components), $m$ and $e$ are the electron mass and charge, $g_{L}$ is the effective Landé factor of the transition $\left(g_{L}=1.67\right.$ for line 1 and $g_{L}=2.5$ for line 2), and $c$ is the speed of light. Given the huge field strength, this approach provides a kind of reality check. 

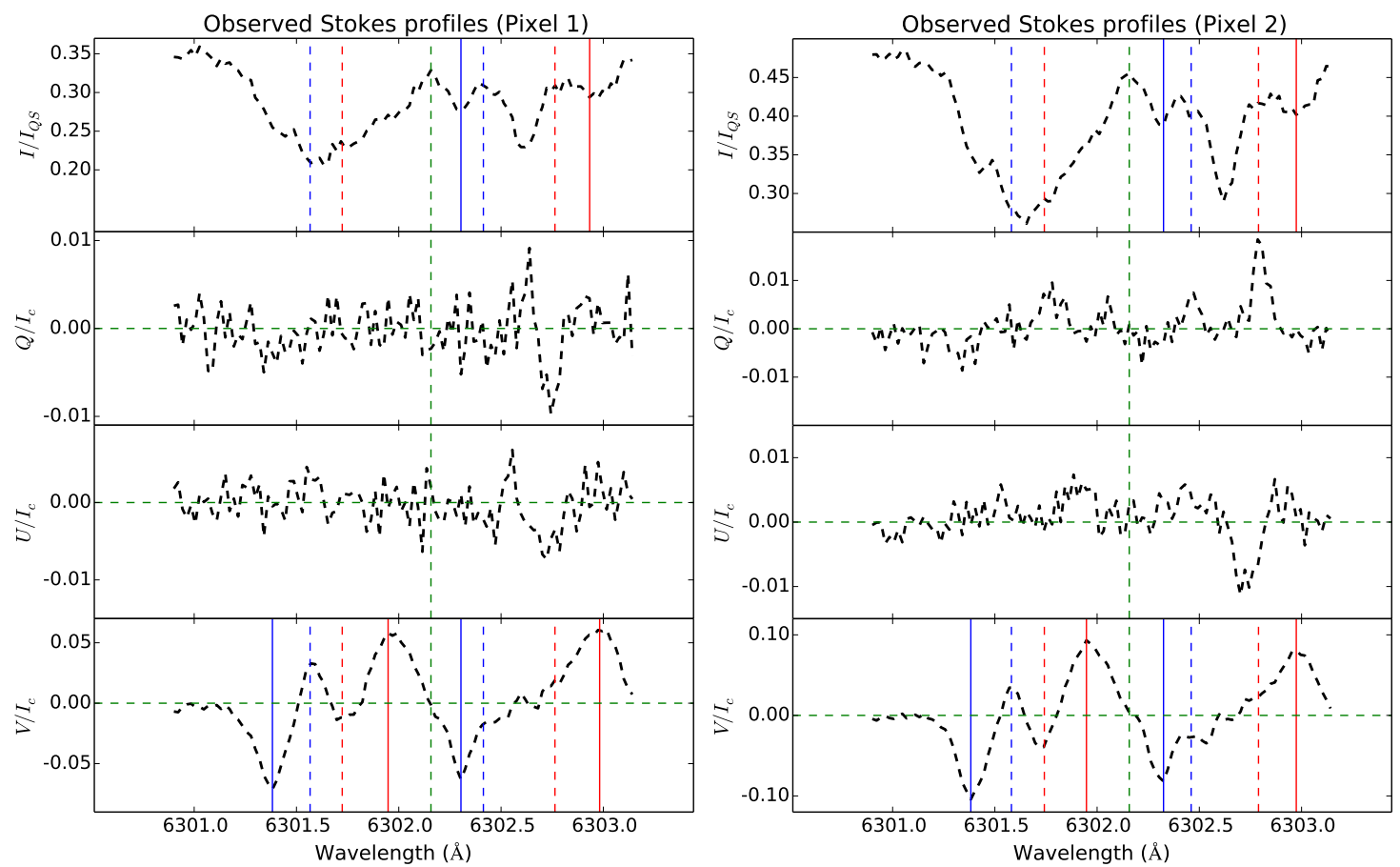

Figure 5.2: Observed Stokes profiles (dashed curves) in two pixels (left: pixel 1, right: pixel 2) located at the inner penumbra of the CEF region (see blue crosses on maps displayed in Fig. 5.1). From top to bottom: Stokes $I, Q, U$ and $V$. The vertical lines in Stokes $I$ and $V$ panels indicate the position of the $\lambda^{-}$(blue) and $\lambda^{+}$(red) used to calculate the magnetic field strength with three different methods, see the main text for a description of the methods: the solid lines in $V$ panels show the splitting derived from method 1; the solid lines in $I$ panels correspond to the line splitting obtained with method 2; and, the dashed lines in both $I$ and $V$ panels indicate the splitting derived from method 3 . The vertical dashed green line shows the wavelength position used to separate the two Fe I lines.

The critical point about using Eq. 5.1 is determining $\lambda^{-}$and $\lambda^{+}$due to the large asymmetries observed in all four Stokes profiles from the LPF. We use three ways: Method 1) Selecting $\lambda^{-}$where Stokes $V$ takes on the largest negative value in the blue wing of the corresponding line, and $\lambda^{+}$where Stokes $V$ is largest in its red wing. Method 2) Placing $\lambda^{-}$ and $\lambda^{+}$in the blue and red wings of the line, respectively, where the two deepest minima of Stokes $I$ away from the central wavelength are found (using line 2 only, since line 1 is insufficiently split). Method 3) Applying the center of gravity method or COG method (Semel 1967, 1970, Rees and Semel 1979, Cauzzi et al. 1993), in which $\lambda^{ \pm}=\lambda_{C O G}^{ \pm}$, where $\lambda_{C O G}^{ \pm}$are the center of gravity wavelengths of the centroids of the right and left circular polarized components, respectively, of the corresponding line, i.e.:

$$
\lambda_{C O G}^{ \pm}=\frac{\int \lambda\left(I_{c}-(I \pm V)\right) d \lambda}{\int\left(I_{c}-(I \pm V)\right) d \lambda},
$$

The position of $\lambda_{C O G}^{ \pm}$and the wavelengths delimiting the integration intervals in Equation 5.2 for each of the Fe I lines, are indicated in Figure 5.2. The resultant field strengths 
obtained with the methods described above are listed in Table 5.1, for both LFPs.

The $B$ values computed with methods 1 and 2 are indeed very large, lying between $\sim 7 \mathrm{kG}$ (obtained from line 2) and $\sim 9.3 \mathrm{kG}$ (from line 1) in both LFPs. On the contrary, the COG method provides considerably smaller field strength values in both LFPs: $B \sim 2.6 \mathrm{kG}$ from line 1 and $3.6-3.9 \mathrm{kG}$ from line 2. The difference between the COG and direct splitting methods likely has to do with the fact that the profiles are not simple Gaussians, but show complex shapes indicating a range of field strengths (of which method 2 samples only the largest), but is also partly due to the non-longitudinal direction of the field (methods 1 and 2 determine field strength, while method 3 only gives the LOS component).

Scatter plots in Figure 5.3 show the results of all three methods applied to all 226 LFPs found in the penumbra. Method 1 returns mainly two different solutions in the two Fe I lines, one of which is centered at $B \sim 3.5 \mathrm{kG}$ in both lines and a second solution that is centered at $B \sim 9 \mathrm{kG}$ for line 1 and at $B \sim 7 \mathrm{kG}$ for line 2 . The fact that method 1 senses two very different field strengths in both lines is a consequence of the complex shape of the multi-lobed Stokes $V$ profiles in the observed spectra, see e.g. Figure 5.4 which displays four different shapes of Stokes $V$ profiles that prevail among the 226 LFPs. For profiles as that shown in Figure 5.4a, method 1 selects the innermost lobes in both lines (given that in this kind of profiles the inner lobes on the red wing of the lines satisfy the criterion used by method 1 to select $\lambda^{+}$), therefore returning field strengths that almost halve the SPINOR 2D inversion result $\left(B_{S P 2} \sim 7.3 \mathrm{kG}\right)$. Similar situations occur in profiles with shapes as shown in Figures 5.4b and 5.4d, where the inner lobes on the red wings for line 1 and line 2, respectively, are the largest. In contrast, in profiles like those shown in Figures 5.4b (line 2), 5.4c (both lines), and 5.4d (line 1), the method selects the most external lobes, therefore computing very large field strengths as displayed in Figure 5.3 (top panels).

The fact that in methods 1 and 3 the results from the 2 lines are not entirely consistent is possibly because the gradients in $B$ and $v_{L O S}$ are not taken into account in Eqs. 5.1 and 5.2. The presence of such gradients is indicated by the asymmetries of the Stokes profiles. Moreover, if there are spatially unresolved areas containing field inhomogeneities (e.g. different field strengths and/or different field polarities either located next to each other or one above the other within the same resolution element), then the resultant line profiles from such pixels will be the summation of all the different components related to the different magnetic fields and hence, to the different thermodynamic conditions in the spectrum, and the number of lobes in the observed Stokes $V$ profiles will depend on the number of unresolved components (e.g., Stenflo 1993). Most Stokes $V$ profiles from the 226 LFPs display more than two lobes, suggesting the presence of different magnetic field components in each of those pixels. Nonetheless, according to methods 1 and 2 , the very large wavelength separation between the most external lobes observed in the Stokes $V$ profiles could still reflect that one of the field components is particularly strong. In such a scenario, an important aspect to consider is if the Paschen-Back effect would play an important role under the presence of such strong fields, i.e. if the splitting of atomic levels caused by such strong external magnetic fields would dominate over the $L S$-coupling (e.g., Chapter 1).

The Paschen-Back regime takes place when $\Delta E_{i k} \gg \mu_{B} g_{L} M B$, where $\Delta E_{i k}$ is the energy difference in the atom between the terms of the multiplet structure, $\mu_{B}$ is the Bohr 

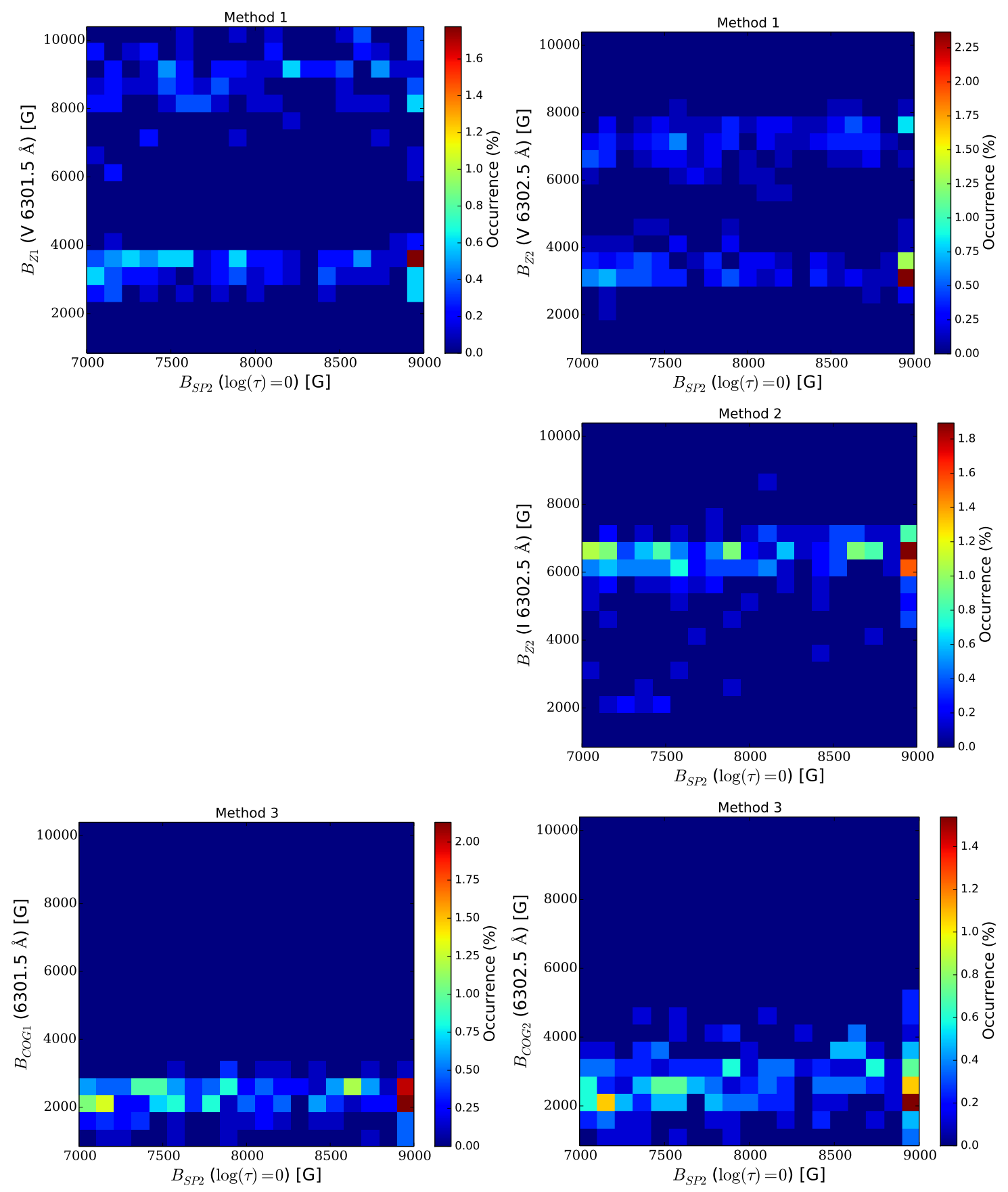

Figure 5.3: Scatter plots of the 226 LFPs where SPINOR 2D returns $B>7 \mathrm{kG}$ at $\log (\tau)=$ $0\left(B_{S P 2}\right)$ versus the respective magnetic field values obtained with each of the 3 alternative methods (Zeeman and COG methods, $B_{Z}$ and $B_{C O G}$ respectively) as described in the text and displayed in Table 5.1. From top to bottom: Method 1,2 and 3 for Fe line at $\lambda=$ $6301.5 \AA$ (subscript 1 , left) and $\lambda=6302.5 \AA$ (subscript 2, right). 


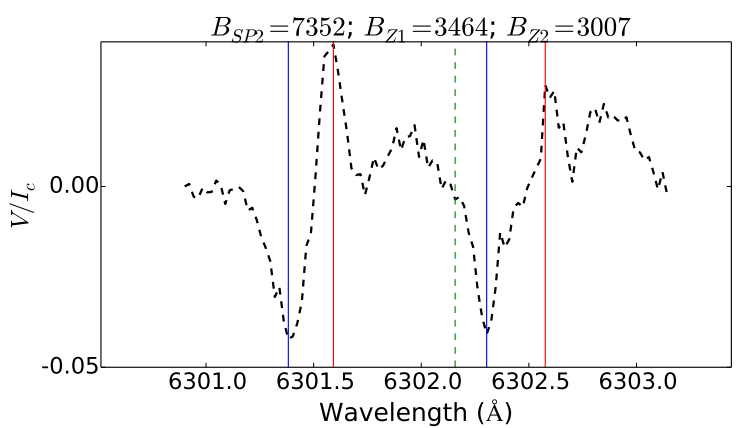

(a)

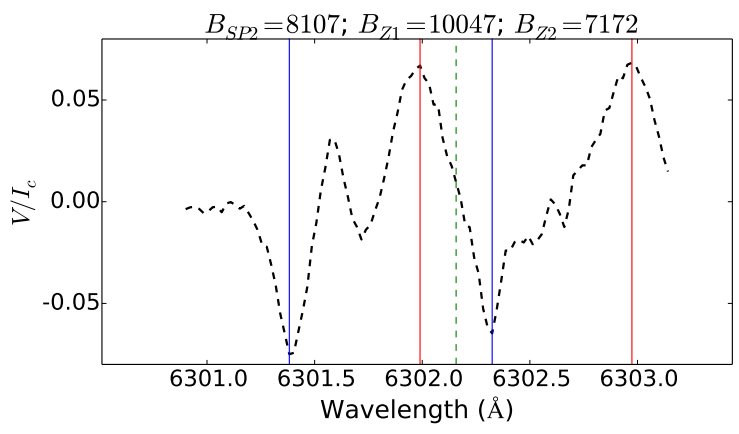

(c)

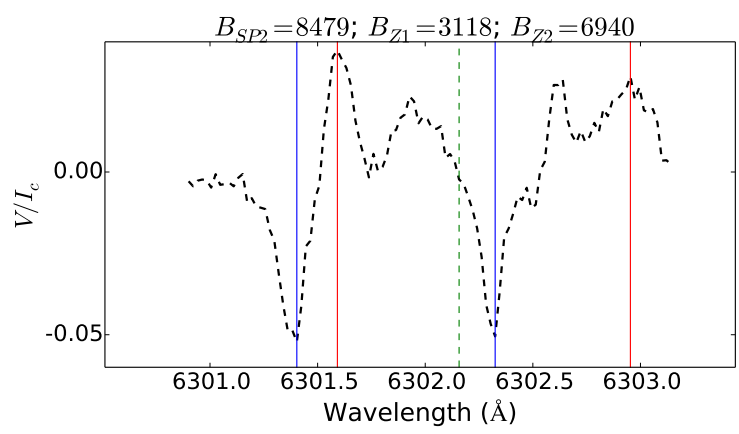

(b)

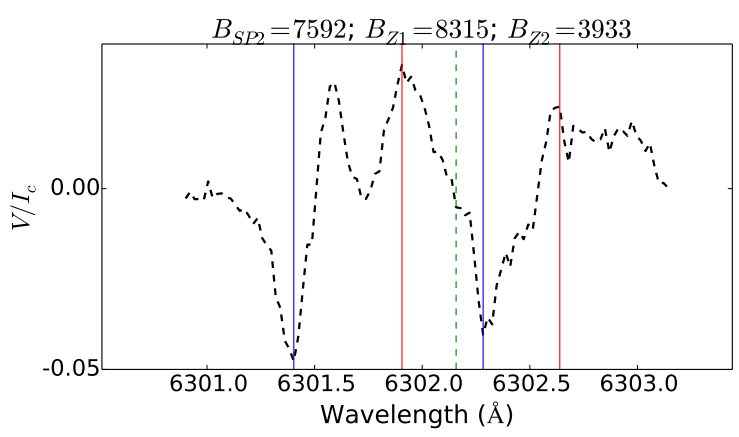

(d)

Figure 5.4: Four examples of observed Stokes $V$ profiles in the LFPs. The vertical lines indicate the position of the $\lambda^{-}$(blue) and $\lambda^{+}$(red) used to calculate the magnetic field strength with method 1 . The headers of the plots indicate the magnetic field strengths according to SPINOR 2D inversions $\left(B_{S P 2}\right)$, according to method 1 in the line at $\lambda=$ $6301.5 \AA\left(B_{Z 1}\right)$ and method 1 in the line at $\lambda=6302.5 \AA\left(B_{Z 2}\right)$.

magneton, $g_{L}$ is the Landé factor, $M$ is the magnetic quantum number, and $B$ is the magnetic field strength. The Fe I ${ }^{5} P_{2}-{ }^{5} D_{2} \lambda=6301.5 \AA$ and the Fe I ${ }^{5} P_{1}-{ }^{5} D_{0} \lambda=6302.5 \AA$ lines belong both to the same multiplet No. 816 (Moore 1945). The minimum energy difference of the lower levels of these lines is $\Delta E_{i k} \approx 0.032 \mathrm{eV}$ and leads to a magnetic field 'threshold value' for Paschen-Back effect of the order of $10^{3} \mathrm{kG}$, a value that is well above the field strength values inferred by methods 1, 2, and the SPINOR 2D inversions. Nonetheless, according with laboratory experiments the Paschen-Back effect actually takes place under the presence of magnetic fields with strengths $10-100 \mathrm{kG}$ when the multiplet splitting energy difference is so small that the two adjacent lines of the same multiplet are separated by a distance of less than $1 \AA$ (Frisch 1963). In the present case, since the analyzed pair of Fe I lines are separated by around $1 \AA$ and the Zeeman splittings being discussed are seemingly very large (corresponding to field strengths that nearly approach $10 \mathrm{kG}$ in some LFPs according to method 1), it is possible that the Paschen-Back effect starts playing some role in the magnetic splitting for those cases. However, even within the Paschen-Back regime, the magnetic field could still be measured with sufficient accuracy according with the laboratory results of Moore (1945).

Another plausible scenario is that the observed multi-lobed Stokes $V$ profiles are produced by two unresolved atmospheric components which display large differences in their Doppler velocity (e.g., Solanki and Montavon 1993, Martínez Pillet 2000, Borrero and 

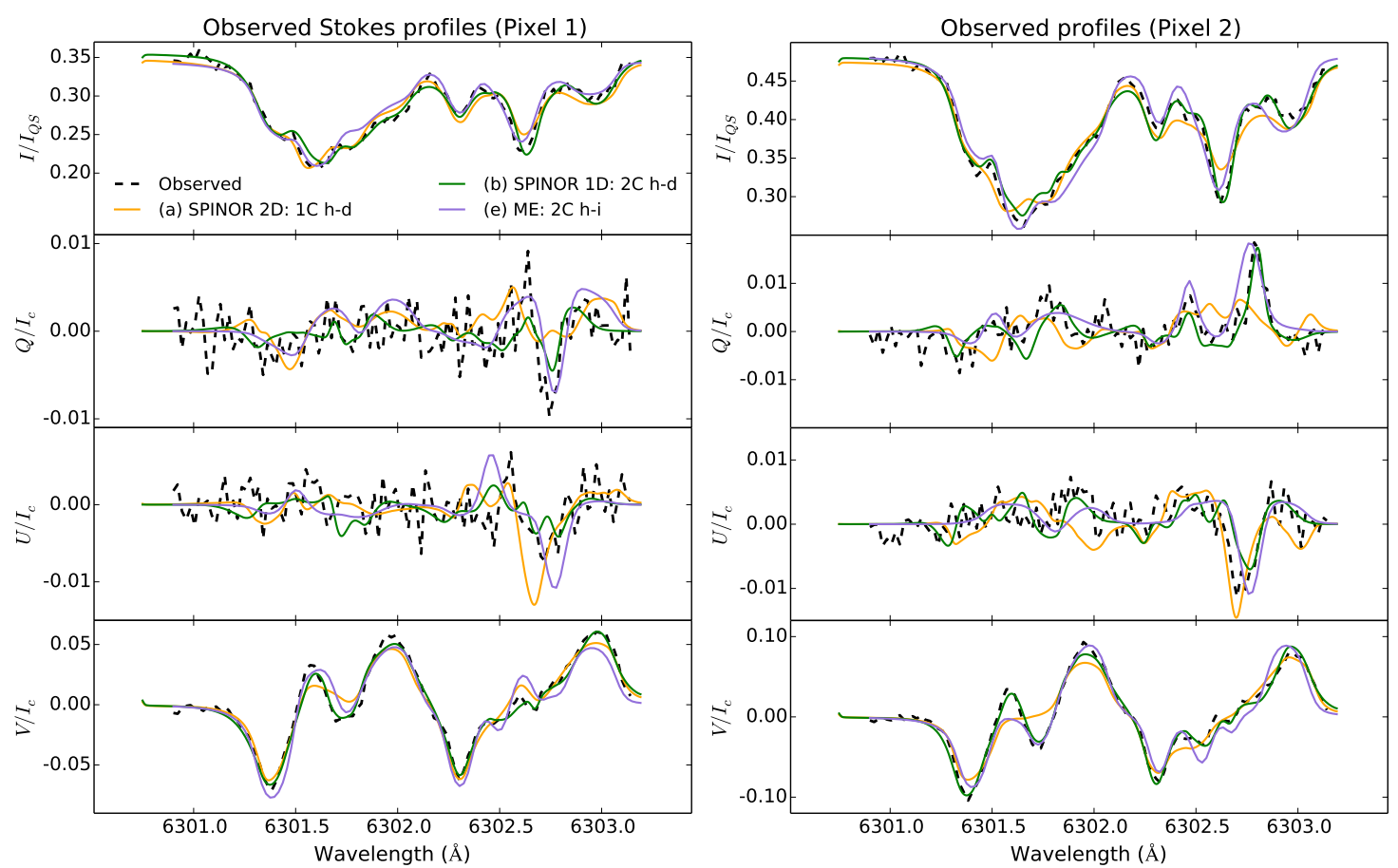

Figure 5.5: Observed Stokes profiles (dashed curves), and their best fits returned by SPINOR 2D (orange curves), SPINOR 1D 2-component height-dependent inversions (green curves) and Milne-Eddington 2-component inversion (purple curves) in the two selected LFPs. Plots are in the same format as plots in Figure 5.2.

Bellot Rubio 2002, Schlichenmaier and Collados 2002, Bellot Rubio et al. 2004). One of the components could be associated with the umbral magnetic field in the sunspot (i.e. nearly at rest) and the second one with the filamentary CEF penumbra (strongly redshifted). This possibility is considered in the following section based on the fact that all the LFPs appear mostly located near, or at the umbral/penumbral edge (see Fig. 5.1).

\subsection{Inversions}

In order to gain more insight into the reliability of the large magnetic field strengths returned by the SPINOR 2D inversion code, we now apply 5 additional inversions, considering two atmospheric components in some of them. It is noteworthy that the inclusion of a second atmospheric component causes the number of free parameters, $n$, to increase to almost twice the number of free parameters in a single component model. While this can and should lead to a much better fit for a complex Stokes profile (lower $\chi^{2}$ ), it also increases the risk of providing artificial (unphysical) results.

In Tables 5.2 and 5.3, we show some of the parameters corresponding to the best fits of the Stokes profiles in the two selected LFPs, obtained from all 6 different inversions. These inversions can be classified into two categories: 

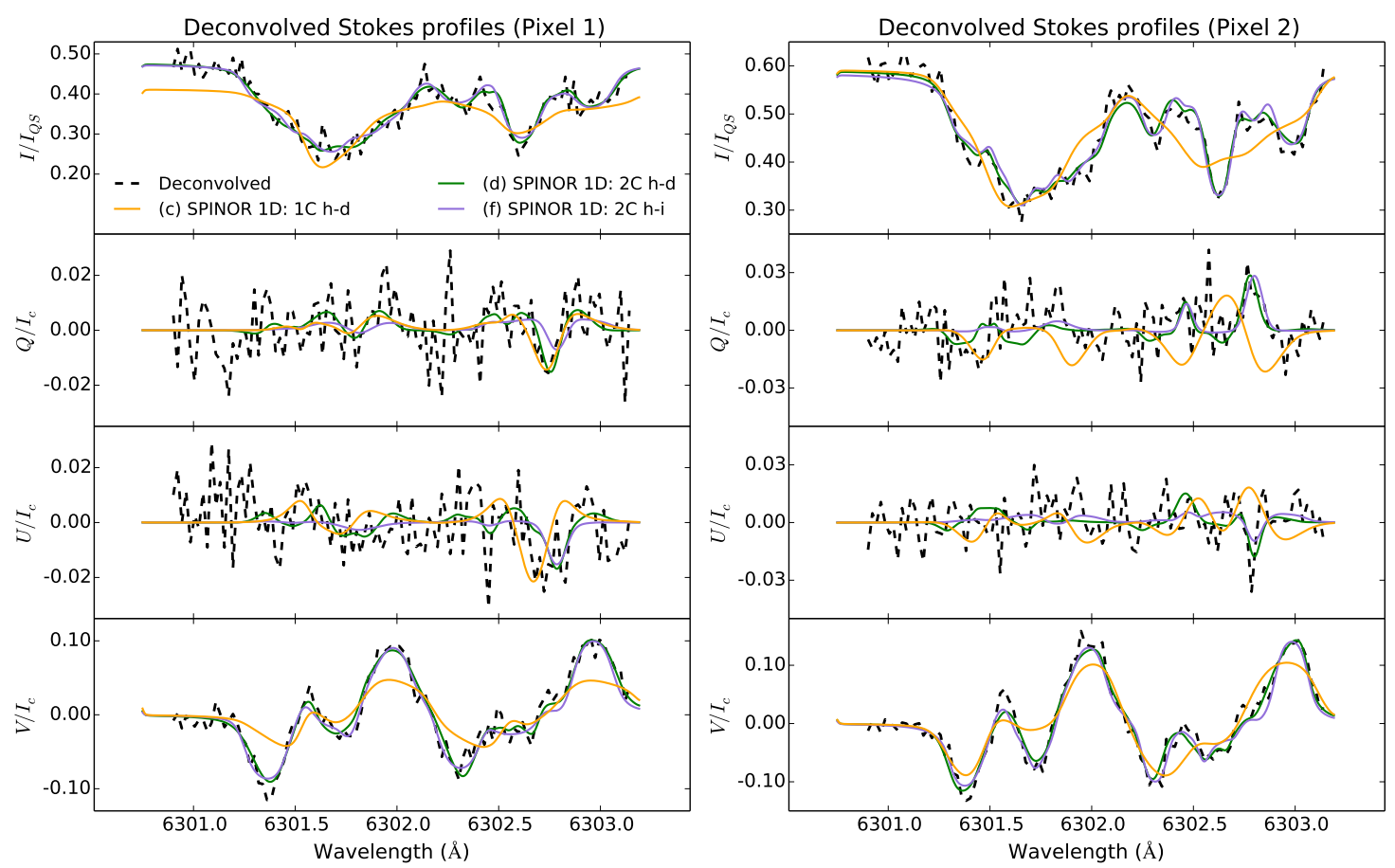

Figure 5.6: Deconvolved Stokes profiles (dashed curve), and their best fits returned by SPINOR 1D inversions, using a single-component height dependent model atmosphere (orange curves), a 2-component height-dependent model (green) and a 2-component height independent model atmosphere (purple curves) for the two selected LFPs. Plots are in the same format as plots in Figure 5.2.

\subsubsection{Height-dependent inversions}

The first category includes height dependent inversions (Table 5.2), i.e., in which all the parameters are allowed to vary with optical depth (with nodes being set at $\log (\tau)=$ $-2.0,-0.8$ and 0), such as (a) SPINOR 2D inversions, with 18 free parameters; (b) SPINOR 1D (Frutiger et al. 2000) 2-component inversions, applied to the observed Stokes profiles (best fits are shown by green curves in Fig. 5.5), with 37 free parameters; (c) SPINOR 1D single-component, applied to the deconvolved Stokes profiles, with 18 free parameters and (d) SPINOR 1D 2-component inversions, applied to the deconvolved Stokes profiles, with 37 free parameters.

Inversions (c) and (d) are intended to resemble the SPINOR 2D technique as far as accounting of the instrumental effects over the observed Stokes profiles is concerned, although the treatment is not as consistent as that by SPINOR 2D. We retrieve the deconvolved Stokes profiles from the spatially degraded observed Stokes profiles by using an effective point-spread function (PSF) (Danilovic et al. 2008) constructed from the pupil function of the 50-cm Hinode SOT (Suematsu et al. 2008) and applying the RichardsonLucy deconvolution method (see Richardson (1972) and Lucy (1974) for details). The resultant deconvolved Stokes profiles in the selected pixels are displayed in Figure 5.6 (black dashed curves) together with their best fits obtained by SPINOR 1D (c) and (d) (orange and green curves in Fig. 5.6, respectively). 


\subsubsection{Height-independent inversions}

The second category corresponds to height independent inversions (Table 5.3), i.e., we assume no variation with optical depth of atmospheric parameters by using (e) a 2components Milne-Eddington inversion (Skumanich and Lites 1987, Lagg et al. 2004, 2009, Borrero et al. 2011) applied to the observed Stokes profiles (best fits are shown by purple curves in Fig. 5.5), with 15 free parameters; and (f) 2-component 1-node SPINOR 1D inversions applied to the deconvolved Stokes profiles (best fits are shown by purple curves in Fig. 5.6), with 17 free parameters.

\subsubsection{Results}

The SPINOR 2D best-fits give $B \sim 8.3 \mathrm{kG}$ at $\log (\tau)=0$ for the profiles in pixel 1 , while $B=8 \mathrm{kG}$ is obtained for both pixels at $\log (\tau)=-0.8$ (see Table 5.2), with $\chi^{2}=14$ and 35 for each fit, respectively. Note that these fits do not succeed in perfectly reproducing the reversed central lobes of the Stokes $V$ profiles observed in both pixels. The deconvolved spectra displayed in Figure 5.6 also show the reversed central lobes in Stokes $V$, which suggests that they are not a result of the mixing of signals due to instrumental effects. Nonetheless, inversions (c) also fail in reproducing the central reversed lobes, providing a much poorer fit than SPINOR 2D $\left(\chi^{2}=44\right.$ and 47 for each pixel, respectively, by taking into account the increased noise in the deconvolved Stokes profiles caused by the deconvolution method itself) and featuring $B \sim 7 \mathrm{kG}$ at $\log (\tau)=0$ in both pixels. Thus, in both cases, the 1-component inversions cannot fit these four-lobe profiles to high precision and return very large field values. Such extreme field values are likely the result of fitting only the external lobes of Stokes $V$ to reproduce the large wavelength separation in terms of the Zeeman splitting.

While the height-dependent 2-component inversions produce better fits to the fourlobe profiles than the 1-component inversions, $\chi^{2}=8$ and 12 for each pixel respectively in inversions (b), and $\chi^{2}=14$ and 18 respectively in inversions (d), they also use a much larger number of free parameters than the 1-component inversions so that a comparison is not straightforward. The components 1 in inversions (b) and (d) give $B \sim 4-4.2 \mathrm{kG}$ and $v_{L O S} \sim 1 \mathrm{~km} \mathrm{~s}^{-1}$ at $\log (\tau)=0$ in both pixels, roughly consistent with the umbral environment in the vicinity of those pixels $(B \sim 4.2 \mathrm{kG}$ at $\log (\tau)=0$, according with SPINOR 2D). Furthermore, the $v_{L O S}$ in the components 1 are sufficiently small at all three atmospheric layers, as expected for umbral environments. In contrast, the components 2 in inversions (b) and (d) give $B \sim 4.4$ and $\sim 4.9 \mathrm{kG}$, respectively, for pixel 1 at $\log (\tau)=0$; and $B \sim 5$ and $5.8 \mathrm{kG}$, respectively, for pixel 2; with $v_{L O S} \gtrsim 16 \mathrm{~km} \mathrm{~s}^{-1}$ and with the filling factors $\alpha$, i.e. the mixing ratio between the 2 components, being slightly larger for the second components. The second component in both pixels could then correspond to the tails of the penumbral filaments harboring the CEF, with a large redshift and stronger fields than in the surrounding umbra; which is qualitatively compatible with the results from SPINOR 2D, but it quantitatively suggests penumbral field strengths of the order of $5 \mathrm{kG}$ (even approaching $6 \mathrm{kG}$ in one pixel).

Therefore, the SPINOR 1D inversion code can find a solution involving 2 components, one of which is strongly wavelength shifted to mimic the seemingly very strongly split spectral line. This nearly halves the field strength, although even in this case, we get $B$ 
values reaching up to nearly $6 \mathrm{kG}$. These are still very large field strengths and are atypical within penumbral environments. They are close to the record measurement of $6.1 \mathrm{kG}$ in sunspot umbrae (Livingston and Harvey 2006). However, due to the large number of free parameters involved, it is difficult to judge if the results they provide are more reliable than those from SPINOR 2D inversions.

As a simple proxy for the quality of the fits, we compare the models by using the Bayesian Information Criterion (BIC; Schwarz 1978), which is based on the crude approximation of Gaussianity of the posterior with respect to the model parameters:

$$
B I C=\chi_{\min }^{2}+n \ln N
$$

where $\chi_{\min }^{2}$ is the merit function of the best-fits to the Stokes profiles in each model, $n$ is the number of free parameters and $N$ is the number of observed points. The computed values of the BIC for each fit are shown in Tables 5.2 and 5.3. The model with the smallest value of the BIC is the preferred one. The height-dependent model preferred by the BIC is SPINOR 2D in both pixels. However, one of the fundamental problems of this criterion is that it penalizes all parameters equally, not taking into account situations in which data do not constrain some parameters (see e.g. Asensio Ramos et al. 2012).

The height-independent 2-component inversions (e) and (f) give nearly identical results to each other in both pixels, with lower field strengths of around $3.5 \mathrm{kG}$ in component 1 , which is almost at rest $\left(v_{L O S}<1 \mathrm{~km} \mathrm{~s}^{-1}\right)$ compared to component 2 in which $B \sim 4 \mathrm{kG}$ and $v_{L O S} \sim 16 \mathrm{~km} \mathrm{~s}^{-1}$. However, the resultant values of $B$ and $v_{L O S}$ given by the two height-independent inversions (e) and (f) generally resemble the results from the height-dependent 2-component inversions (b) and (d) at $\log (\tau)=-0.8$. This is not surprising since the sensitivity to $v_{L O S}$ and $B$ perturbations is higher at $\log (\tau)=-0.8$ than at $\log (\tau)=0$ for the Fe I $6302.5 \AA$ line (see, e.g., response functions computed by Cabrera Solana et al. (2005)). Even if the absorption line is not formed at a single depth, the height-independent inversions mainly provide information on the physical conditions prevailing at depths at which the line is more sensitive. Thus, if stronger magnetic fields are present in deeper layers of the solar atmosphere (e.g. at $\log (\tau)=0$ ), as suggested by the results of inversions (a), (b), (c) and (d), they cannot be retrieved by inverting the Stokes profiles of the current wavelength range with a height-independent inversion technique only.

The BIC values obtained for inversions (e) and (f) are only slightly better than the ones obtained for SPINOR 2D in both pixels. Even if they both succeed in capturing many relevant aspects of all four Stokes profiles (despite the increased noise in the deconvolved spectra), it is very unlikely that the physical parameters in the pixels of interest display no gradients with height. Generally in sunspots, one would rather expect large gradients with height of the physical parameters (particularly of the magnetic field) as supported by MHD simulations. In such cases, height-dependent inversions provide a more appropriate model atmosphere. The SPINOR 2D inversions are the most reliable model in this sense, since they take into account the height stratification of the physical parameters while keeping a good balance between the quality of the fit and the number of free parameters in the model, according to the obtained BIC values in the two studied pixels with peculiar spectra. 


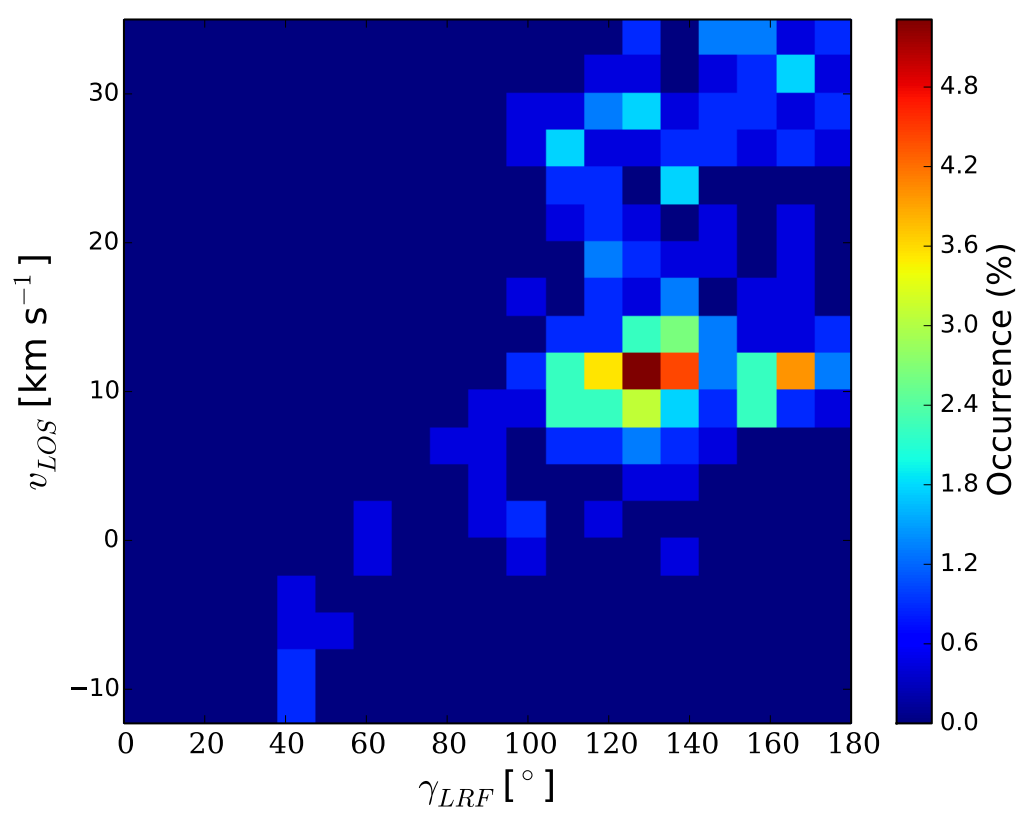

Figure 5.7: Scatter plot of the magnetic field inclination in the LRF $\gamma_{L R F}$ (after removal of the field azimuth ambiguity through the NPFC method) versus the LOS velocity $v_{L O S}$, from SPINOR 2D at $\log (\tau)=0$, for the 226 LFPs.

\subsection{Discussion}

Inversion techniques are currently the most powerful tools to infer the physical properties of the solar atmosphere from polarization line profiles, being able to provide reliable and robust results according to some numerical tests (e.g., Ruiz Cobo 2007). There are several different inversion techniques in the literature, each of them with their own advantages and shortcomings, which largely depend on the problem. Certainly, after any Stokes inversion the results need always to be validated and one needs to be aware that the resulting model atmosphere is not necessarily the real one since the solution might not be unique. Nonetheless, as stated by Sabatier (2000), by means of Stokes inversions it is generally possible to retrieve as much information as possible for a model which is proposed to represent the system in the real world.

The existence of $B>7 \mathrm{kG}$ in the inner penumbra of a sunspot would require an unusually deep Wilson depression to be consistent with idealized magnetohydrostatic models of sunspots (Livingston and Harvey 2006). However, field strengths larger than $7 \mathrm{kG}$ in penumbral environments have previously been reported by van Noort et al. (2013). They obtain such large field strengths in supersonic downflow regions in the peripheric penumbra of a sunspot, also by using SPINOR 2D inversions. Nonetheless, they observe $B>7$ $\mathrm{kG}$ only in the deepest layer $(\log (\tau)=0)$ and obtain a good agreement between the inversion results and a MURaM simulation of a sunspot by Rempel (2012b). They propose a scenario in which the high magnetic field values are the result of a field intensification in the deep photosphere due to the interaction of the supersonic downflows with an external magnetic barrier (e.g., with a plage region). Such a scenario could also be valid for the present observations, with the umbral field playing the role of the magnetic barrier.

According to our SPINOR 2D results, most of the magnetic fields whose strength is 


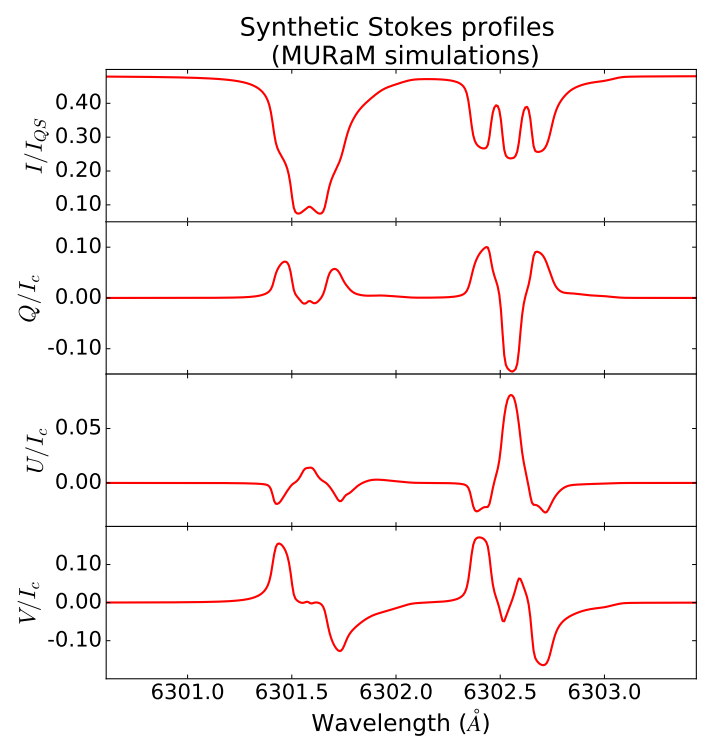

(a)

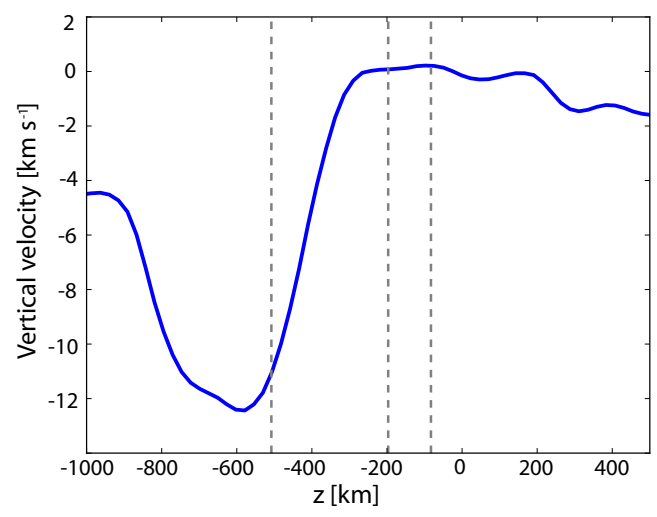

(c)

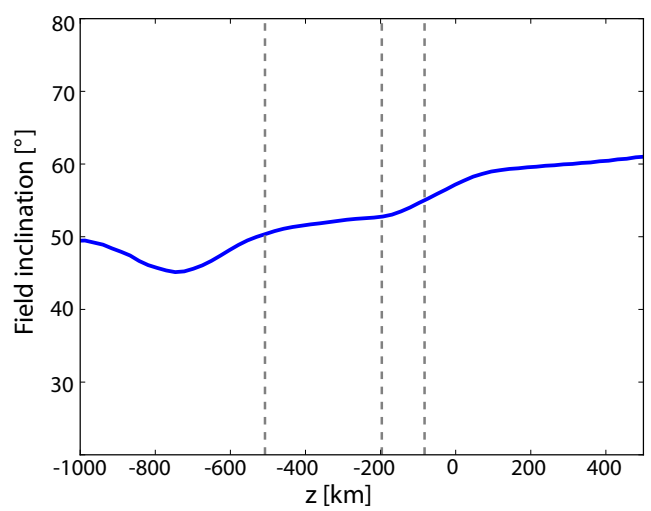

(e)

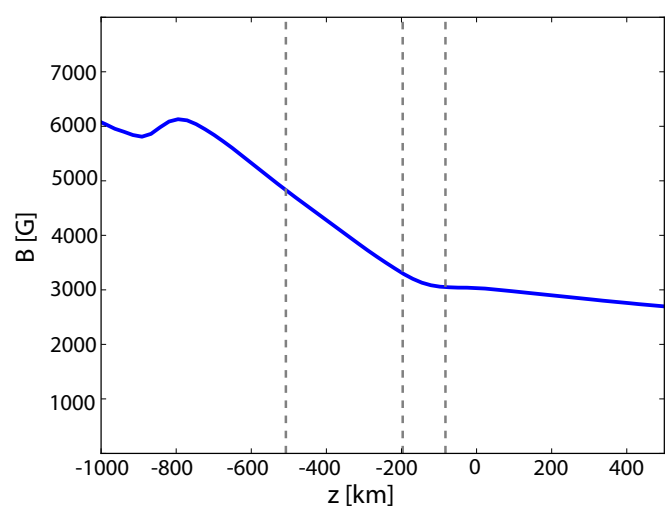

(b)

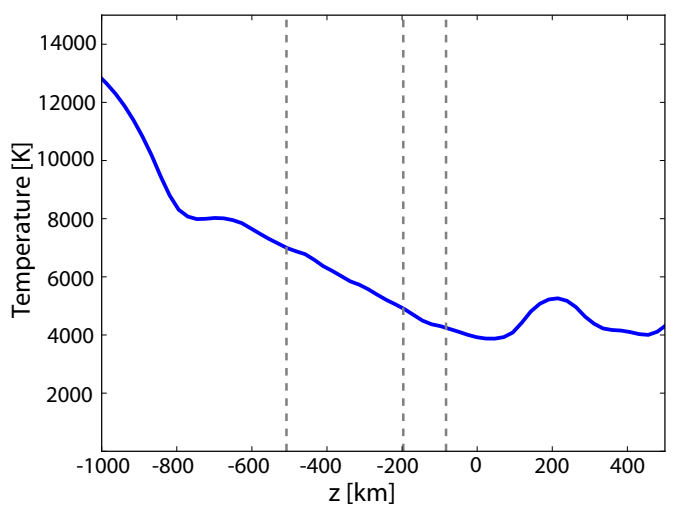

(d)

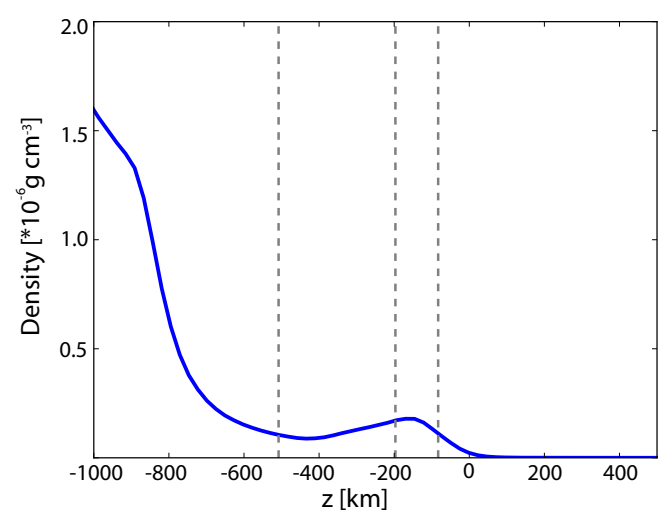

(f)

Figure 5.8: (a) A set of emergent synthetic Stokes profiles in the MURaM sunspot simulation presented in Chapter 4 at the location of a supersonic downflow in the tail of a CEF-carrying filament. The spectra were synthesized by using the SPINOR code (STOPRO routines). (b) Vertical structure of the magnetic field strength, (c) the vertical flow velocity (negative values indicate downflows), (d) the temperature, (e) the field inclination, and (f) the gas density at the location of the grid-cell containing the supersonic downflows from the MURaM simulation. Vertical dashed lines on panels (b)-(f) indicate (from left to right) the local depth of the $\log (\tau)=0,-0.8$, and -2 respectively. 
above $7 \mathrm{kG}$ are nearly vertical and have the same polarity as the umbra, which is negative. The scatter-plot in Figure 5.7 shows the magnetic field inclination angle in the localreference-frame (LRF) after the azimuthal disambiguation was resolved with the NPFC method (see Chapter 3) versus the LOS velocity at $\log (\tau)=0$, for all the 226 LFPs in the CEF penumbra. Most of the LFPs (see main population in Fig. 5.7) appear associated with supersonic LOS velocities, i.e. around $v_{L O S}=10 \mathrm{~km} \mathrm{~s}^{-1}$ (the sound speed is around $C_{s} \sim 5-8 \mathrm{~km} \mathrm{~s}^{-1}$ in penumbrae, e.g., van Noort et al. 2013) and even larger than 20 $\mathrm{km} \mathrm{s}^{-1}$ in some pixels. The magnetic field in those regions is largely vertical (the peak of the LFPs distribution occurs near $\gamma_{L R F}=140^{\circ}$ ). Therefore, under the assumption of field-aligned flows, those pixels likely contain downflows. Moreover, the regions in the sunspot where $B>5 \mathrm{kG}$ are also associated with supersonic LOS flow velocities (see, e.g., Fig. 3.5a where regions with $B>5 \mathrm{kG}$ are enclosed within cyan contours and appear co-located with the regions where $v_{L O S}>9 \mathrm{~km} \mathrm{~s}^{-1}$ which are surrounded by red contours). As displayed in Figure 5.1, the LFPs with $B>7 \mathrm{kG}$ (yellow pixels) are surrounded by those weaker fields, which are still in excess of $5 \mathrm{kG}$ (red pixels), in a supersonic flow environment. Thus, a very low density of the supersonic downflowing material could also explain the possible observation of unusually strong magnetic fields in the penumbra, since it would cause the optical depth layers to be strongly depressed. In addition, their close vicinity to the umbral field also plays a role. This is in agreement with MHD simulations of counter-Evershed flows (Siu-Tapia et al. 2018, and Chapter 4), which show a nearly $500 \mathrm{~km}$ depressed $\tau=1$ surface (with respect to its average height in the simulated penumbra) at the tails of the CEF-carrying filaments, where the supersonic downflowing material becomes a very low density gas (see also Fig. 5.8). Consequently, the magnetic field strength is of the order of $5 \mathrm{kG}$ at those heights.

In a rather simple and quick attempt to quantify the effect of the 3D atmospheric structure and magnetic field on the profiles of the analyzed spectral lines, we employed the forward part of the SPINOR code (STOPRO) to solve the radiative transfer equation in the MURaM cubes analyzed in Chapter 4. Figure 5.8a shows the emergent synthetic spectra from a vertical column located close to the inner edge of the simulated penumbra and which, at the $\log (\tau)=0$ level, intersects with the tail of a CEF-carrying filament and contains supersonic downflows at that height. Similarly to the observed Stokes profiles for the pair of Fe I lines, the synthetic Stokes profiles in the simulations are highly asymmetric, display large redshifts, and show multi-lobed Stokes $V$ profiles. Such complex shapes are mainly the result of the large vertical gradients in all the atmospheric quantities and the magnetic field structure. In particular, the stratification of the field strength (Figure 5.8b), the flow velocity (Figure 5.8c), and field inclination (Figure 5.8d), qualitatively resemble the SPINOR 2D results in the LFPs (cf. Table 5.2), i.e., while the field strength and downflow speeds increase with depth, the field inclination increases with height. Such strong magnetic fields in the simulations (nearly $5 \mathrm{kG}$ at the $\operatorname{local} \log (\tau)=0$ level) are mainly due to the influence of the neighboring umbral field and the highly depressed surfaces of constant optical depth, but there is also a local net intensification of the field that can be associated to the converging aspect of the supersonic downflows which lead to a compression and intensification of the vertical magnetic field component in the inner penumbra (similarly, at the sinks of the NEF, close to the outer penumbral boundary, the vertical field can also be locally intensified by the downward advection of magnetic flux concentrations caused by the supersonic downflows). Therefore, given the extreme 
characteristics of the observed Stokes profiles associated with the sinks of the CEF in the LFPs, we finally cannot easily discard the possibility that we are dealing with actual observations of $B \sim 7 \mathrm{kG}$ and even larger in regions of supersonic downflowing material with very low densities, where similar mechanisms to those occurring in the simulations (see Chapter 4) might explain their origin.

The major strength of SPINOR 2D lies on its simultaneous coupled inversion of all the pixels to self-consistently take into account the influence of straylight from neighboring pixels. This approach is able to reproduce complex multi-lobed profiles with a simple, one-component atmosphere per pixel, keeping an acceptable number of free parameters which significantly enhance the reliability and the robustness of the inversion results. However, we have seen that the highly complex observed Stokes profiles cannot be perfectly reproduced with any of the presented inversion techniques without almost doubling the number of free parameters. The inherent complexity of these profiles may involve physical aspects that are not considered within the assumptions and approximations made by the inversion codes, which could lead to significant errors in the returned values.

For completeness, it would be interesting to test and validate the performance of the different inversions under the presence of strong photospheric magnetic fields by applying them to the synthetic spectra from the MHD simulation presented in Chapter 4 and in "potentially" strong field regions in other sunspots, e.g. in the peripheral penumbral downflows from the spot analyzed by van Noort et al. (2013). Likewise, the effects of such possible superstrong magnetic fields on the behavior of the Stokes profiles need to be taken into account by the inversion codes (e.g. the calculation of full Stokes spectra in the Paschen-Back regime). This will be the basis of a future study.

Finally, the field estimations performed by means of the Zeeman splitting and the COG method (methods 1, 2, and 3) provide highly inconsistent and likely erroneous results. Unfortunately, all these methods are unable to take into account the errors from the instrumental as well as from the data reduction procedures. Moreover, methods 1 and 2 are reliable on ideal cases only, as when they are applied to single-component profiles produced by homogeneous magnetic fields, which is clearly not the case for the LFPs with highly asymmetric and multi-lobed Stokes profiles. As a consequence, the possibility of two (horizontally) unresolved structures with a large velocity difference cannot be ruled out either, in spite of the shortcomings of the 2-component height-dependent and height-independent inversions. Nevertheless, unusually strong penumbral magnetic fields (5-6 kG) also show up as the more plausible physical solution in those models. 
Height-dependent inversions

\begin{tabular}{|c|c|c|c|c|c|c|c|c|c|c|}
\hline inversion technique & $n$ & $\mathrm{P}$ & $\mathrm{C}$ & $\log (\tau)$ & $\begin{array}{l}B \\
{[\mathrm{kG}]}\end{array}$ & $\begin{array}{l}\gamma \\
{\left[{ }^{\circ}\right]}\end{array}$ & $\begin{array}{l}v_{L O S} \\
{\left[\mathrm{~km} \mathrm{~s}^{-1}\right]}\end{array}$ & $\chi^{2}$ & $\alpha$ & $\mathrm{BIC}$ \\
\hline \multirow{6}{*}{$\begin{array}{l}\text { (a) SPINOR 2D } \\
\text { (observed) }\end{array}$} & \multirow{6}{*}{18} & \multirow{3}{*}{1} & \multirow{3}{*}{1} & -2.0 & 6.4 & 141 & 5.6 & \multirow{3}{*}{14} & \multirow{3}{*}{1} & \multirow{3}{*}{98} \\
\hline & & & & -0.8 & 8.0 & 148 & 8.3 & & & \\
\hline & & & & 0.0 & 8.3 & 145 & 9.3 & & & \\
\hline & & \multirow{3}{*}{2} & \multirow{3}{*}{1} & -2.0 & 7.0 & 139 & 7.9 & \multirow{3}{*}{35} & \multirow{3}{*}{1} & \multirow{3}{*}{119} \\
\hline & & & & -0.8 & 8.0 & 146 & 9.3 & & & \\
\hline & & & & 0.0 & 7.4 & 173 & 12.1 & & & \\
\hline \multirow{12}{*}{$\begin{array}{l}\text { (b) SPINOR 1D } \\
\text { (observed) }\end{array}$} & \multirow{12}{*}{37} & \multirow{6}{*}{1} & & -2.0 & 3.6 & 166 & 0.2 & \multirow{6}{*}{8} & \multirow{3}{*}{0.48} & \multirow{6}{*}{181} \\
\hline & & & 1 & -0.8 & 4.0 & 155 & 0.4 & & & \\
\hline & & & & 0.0 & 4.2 & 146 & 0.5 & & & \\
\hline & & & & -2.0 & 2.9 & 163 & 15.5 & & \multirow{3}{*}{0.52} & \\
\hline & & & 2 & -0.8 & 3.9 & 177 & 17.5 & & & \\
\hline & & & & 0.0 & 4.4 & 179 & 17.4 & & & \\
\hline & & \multirow{6}{*}{2} & & -2.0 & 3.6 & 159 & 0.1 & \multirow{6}{*}{12} & \multirow{3}{*}{0.35} & \multirow{6}{*}{185} \\
\hline & & & 1 & -0.8 & 4.0 & 154 & 0.3 & & & \\
\hline & & & & 0.0 & 4.2 & 144 & 1.1 & & & \\
\hline & & & & -2.0 & 2.3 & 152 & 14.3 & & \multirow{3}{*}{0.65} & \\
\hline & & & 2 & -0.8 & 4.0 & 169 & 17.0 & & & \\
\hline & & & & 0.0 & 5.1 & 179 & 16.0 & & & \\
\hline \multirow{6}{*}{$\begin{array}{l}\text { (c) SPINOR 1D } \\
\text { (deconvolved) }\end{array}$} & & & & -2.0 & 2.5 & 100 & 9.8 & & & \\
\hline & & 1 & 1 & -0.8 & 7.4 & 179 & 12.7 & 44 & 1 & 128 \\
\hline & 18 & & & 0.0 & 7.2 & 169 & 4.7 & & & \\
\hline & 18 & & & -2.0 & 2.9 & 67 & 8.5 & & & \\
\hline & & 2 & 1 & -0.8 & 5.2 & 178 & 11.4 & 47 & 1 & 131 \\
\hline & & & & 0.0 & 6.6 & 132 & 6.7 & & & \\
\hline & & & & -2.0 & 3.0 & 159 & 0.0 & & & \\
\hline & & & 1 & -0.8 & 3.5 & 170 & 0.5 & & 0.42 & \\
\hline & & 1 & & 0.0 & 4.0 & 178 & 1.0 & 14 & & 187 \\
\hline & & 1 & & -2.0 & 2.8 & 144 & 14.4 & 14 & & 181 \\
\hline & & & 2 & -0.8 & 4.0 & 171 & 16.5 & & 0.58 & \\
\hline (d) SPINOR 1D & 37 & & & 0.0 & 4.9 & 179 & 17.7 & & & \\
\hline (deconvolved) & 31 & & & -2.0 & 3.2 & 157 & 0.2 & & & \\
\hline & & & 1 & -0.8 & 3.8 & 157 & 0.6 & & 0.39 & \\
\hline & & 2 & & 0.0 & 4.2 & 156 & 1.1 & 18 & & 191 \\
\hline & & 2 & & -2.0 & 1.7 & 165 & 15.6 & 18 & & 191 \\
\hline & & & 2 & -0.8 & 4.0 & 172 & 17.1 & & 0.61 & \\
\hline & & & & 0.0 & 5.8 & 176 & 16.4 & & & \\
\hline
\end{tabular}

Table 5.2: Parameters resulting from 4 different height dependent inversions which were applied to the two sets of observed Stokes profiles ((a) and (b)) and to their corresponding deconvolved Stokes profiles ((c) and (d)) from the two selected LFPs. From left to right: number of free parameters $n$, pixel identification number $\mathrm{P}$, atmospheric component $\mathrm{C}$, optical depth node $\log (\tau)$, field strength $B$, field inclination $\gamma$, LOS velocity $v_{L O S}$, merit function $\chi^{2}$, filling factor $\alpha$ for each component, and BIC value. 
Height-independent inversions

\begin{tabular}{|c|c|c|c|c|c|c|c|c|c|}
\hline inversion technique & $n$ & $\mathrm{P}$ & $\mathrm{C}$ & $\begin{array}{l}B \\
{[\mathrm{kG}]}\end{array}$ & $\begin{array}{l}\gamma \\
{\left[{ }^{\circ}\right]} \\
\end{array}$ & $\begin{array}{l}v_{L O S} \\
{\left[\mathrm{~km} \mathrm{~s}^{-1}\right]}\end{array}$ & $\chi^{2}$ & $\alpha$ & $\mathrm{BIC}$ \\
\hline \multirow{4}{*}{$\begin{array}{l}\text { (e) ME } \\
\text { (observed) }\end{array}$} & \multirow{4}{*}{15} & \multirow{2}{*}{1} & 1 & 3.4 & 169 & 0.0 & \multirow{2}{*}{22} & 0.66 & \multirow{2}{*}{92} \\
\hline & & & 2 & 4.1 & 162 & 15.5 & & 0.34 & \\
\hline & & \multirow{2}{*}{2} & 1 & 3.4 & 165 & 0.5 & \multirow{2}{*}{34} & 0.55 & \multirow{2}{*}{104} \\
\hline & & & 2 & 3.9 & 161 & 15.3 & & 0.45 & \\
\hline \multirow{4}{*}{$\begin{array}{l}\text { (f) SPINOR 1D } \\
\text { (deconvolved) }\end{array}$} & \multirow{4}{*}{17} & \multirow{2}{*}{1} & 1 & 3.5 & 173 & 0.0 & \multirow{2}{*}{15} & 0.32 & \multirow{2}{*}{95} \\
\hline & & & 2 & 3.9 & 165 & 15.9 & & 0.68 & \\
\hline & & \multirow{2}{*}{2} & 1 & 3.5 & 171 & 0.5 & \multirow{2}{*}{18} & 0.35 & \multirow{2}{*}{98} \\
\hline & & & 2 & 4.0 & 166 & 16.6 & & 0.65 & \\
\hline
\end{tabular}

Table 5.3: Parameters resulting from two height independent inversions: (e) twocomponents Milne-Eddington inversions applied to the two sets of observed Stokes profiles (black dashed lines in Fig. 5.5) and, (f) two-components SPINOR 1D inversions applied to the corresponding deconvolved Stokes profiles (black dashed lines in Fig. 5.6) of the two selected LFPs (blue markers on Fig. 5.1). Columns are in the same format as in Table 5.2. 



\section{Conclusions and Outlook}

This final chapter provides a brief summary and the general conclusions derived from the main findings presented in Chapters 3, 4, and 5, as well as an outlook.

\subsection{Brief summary and conclusions}

Well-developed penumbrae of sunspots harbor a distinctive gas outflow in the photosphere, known as the Evershed flow, whose origin has been a long-standing open question in sunspot physics since its discovery in 1909. Recent high-resolution observations have disclosed many details of this flow and, abreast with the results of realistic sunspot simulations, are converging towards a possible scenario to explain its nature.

More recently, the existence of counter-Evershed flows (photospheric inflows) in welldeveloped penumbrae have been detected. The small number of observations of this type of penumbral flows is perhaps a consequence of their rare occurrence. Additionally, the continuum intensity maps of these counter-Evershed flows (CEFs) are almost indistinguishable from the normal-Evershed flows (NEFs). Therefore, little is known about their nature, characteristics and their relation with the filamentary structure of penumbrae.

In this thesis, we have studied the magnetic field and flow characteristics in observed and simulated sunspot penumbrae that host CEFs in some of their penumbral filaments at photospheric heights. The origins of both, NEF and CEF were investigated, and their physical characteristics as well as their fine structures were compared. Therefore, our results provide new information on the fairly rare and poorly understood CEFs, but also strengthen our understanding of the NEF, as well as of the penumbral morphology and dynamics.

- In Chapter 3 (whose content has been published in the paper by Siu-Tapia et al. 2017), we presented SPINOR 2D inversions of spectropolarimetric observations in active region NOAA 10930 recorded with the SOT/SP instrument onboard the Hinode space observatory. By analyzing the general characteristics as well as the polarization signals in the observed Stokes profiles, we found that the penumbra of the main sunspot in this active region displayed a large sector harboring the most prominent CEF ever reported. According to the results of our inversions, those CEFs reach supersonic LOS speeds, in excess of $9 \mathrm{~km} \mathrm{~s}^{-1}$, towards the inner penumbral boundary. Furthermore, we found that the CEF was confined along an array of reversed penumbral filaments, whose heads (filament endpoints hosting the uplowing hot material) were located near the outer penumbral boundary, while their tails (filament endpoints hosting downflowing cooler gas) were localized close to 
the umbral-penumbral edge in regions of enhanced magnetic field strength (with respect to the surrounding penumbral and umbral magnetic environment). After solving the azimuthal ambiguity of the magnetic field, we could construct a picture of the magnetic field configuration in both, the CEF-carrying and the NEF-carrying penumbral filaments:

- This picture is consistent with the penumbral models that describe penumbral filaments as bundles of shallow $\cap$-shaped magnetic field lines carrying fieldaligned flows.

- Both, the NEF-carrying filaments and the CEF-carrying filaments are qualitatively similar in the sense that, in both cases, the gas flows from the heads to the tails while displaying a gradual decrease in temperature towards the tails, but presenting a secondary temperature peak in the tails.

- Such a secondary temperature increase in the tails was first found by Tiwari et al. (2013) in the NEF-carrying filaments and might be a consequence of the depressed optical depth levels in the tails due to the presence of concentrated downflows of cooler and less dense gas, which allows for the observation of the hotter lower layers in the atmosphere; but it can also be due to the formation of shocks due to the supersonic speeds of the downflows in the case of the tails of the CEF-carrying filaments.

- Both, NEF- and CEF-carrying filaments present downflow lanes along their sides.

- We found enhanced vertical fields in the tails of both types of filaments: $2-2.5 \mathrm{kG}$, on average, with opposite polarity to the umbral field in the tails of the NEF-carrying filaments; and $~ 4.5 \mathrm{kG}$, on average, with the same polarity as the umbral field in the tails of the CEF-carrying filaments.

Therefore, the magnetic, thermal, and flow structures in both types of filaments display characteristics that are compatible with the magneto-convective driver scenario (the thermal gradient between heads and tails, and the signatures of lateral overturning convection support this picture) as well as with the siphon flow scenario (the enhanced fields found in the tails suggest a gas pressure difference driving the gas outward/inward in NEF-/CEF-carrying filaments and support this picture). Nonetheless, a conclusion about the main driving forces of the flows could not be drawn from information obtained on constant optical depth surfaces only.

According to the SP scans from Hinode, the CEF lasted during $\sim 2$ days in the anomalous part of the penumbra, although none of those scans could capture the initiation of the CEF. The temporal evolution of the active region as observed in the Hinode G-band images suggests that the CEF-carrying penumbral sector could have developed from a penumbral-like connection between the main sunspot and a pre-existing adjacent smaller spot of opposite polarity. During such an initial connection, the penumbral filaments in the region of interest appeared to emerge from the small spot and to gradually grow with time at the expenses of the magnetic flux present inside the small spot, which finally seemed to lead to the spot's extinction. Therefore, it is possible that such a 'partial penumbra' was carrying a NEF (from 
the small umbra outwards, i.e. towards the umbra of the main sunspot) which was interpreted as a CEF with respect to the main sunspot once the opposite polarity spot disappeared.

- In Chapter 4 (which largely follows a manuscript accepted by ApJ, Siu-Tapia et al. 2018), we have presented an in-depth analysis of a high-resolution magnetohydrodynamical simulation of a sunspot by Rempel (2015), whose well-developed penumbra harbors localized CEFs. In order to compare the physical differences between NEF and CEF, we have analyzed the fine structure of individual flow channels. Also, in order to unmask the responsible driving forces of the large-scale flows in the simulated penumbra (outflows and inflows) we have analyzed the different terms in the kinetic energy equation as well as the magnetic connectivity of the field lines in the flow channels. We also analyzed the different terms of the induction equation in order to obtain insight into how the different components of the magnetic field vector are affected by the presence of strong flows in the penumbra. The main conclusions from these analysis are as follows:

- Both, NEF and CEF are driven in a thin boundary layer located near the solar surface and display a filamentary structure.

- The location within the penumbra as well as the radial extension of the upflow cells are different in NEF- and CEF-carrying filaments. These two aspects influence the driving mechanisms of both flows.

- At the sources of both, NEF and CEF, the loss of buoyancy due to radiative cooling contributes only partly to the deceleration of upflows. This partial contribution is larger for the CEF than for the NEF.

- The NEF displays characteristics that are compatible with overturning convection occurring in a strongly inclined magnetic field, i.e. in a field almost parallel to the solar surface. The analysis of its driving forces showed that, at the source locations, the flow is deflected radially by the highly inclined magnetic field in the penumbra through the Lorentz force. However, almost no work is done by the Lorentz force. The radial outward acceleration of the fluid is a result of the mass flux conservation and the strong upward decrease of the gas density in the near surface layers. The presence of weak downflows at the sides of filaments also support this scenario.

- CEFs are driven mainly by radial pressure gradients in the near-surface layers of the penumbra and display a consistent field-aligned inward motion with a systematic pressure perturbation along the field lines. Therefore, CEFs can be well described as siphon flows occurring along magnetic flux tubes. The vertical increase of the radial field component in the outer and middle penumbra cause the radial Lorentz force to also contribute (although to a lesser degree) to the inward radial driving of the flow.

- CEFs have short lifetimes in this simulation ( 10 solar hours), and show a large variability in their integrated inward mass flux due to the intermittent appearance of CEFs with much shorter lifetimes. In contrast, the NEF shows up as a robust feature in the penumbra, which is consistent with the observations. 
- Both, NEF- and CEF-carrying filaments display characteristics that are similar to the observations analyzed in Chapter 3, i.e. in this simulation: a) Supersonic downflows are observed in the sink locations, i.e. the tails of both, NEF- and CEF-carrying filaments. b) Secondary peaks in the radial temperature profiles are found at the sink locations of both, NEF and CEF filaments, which are related to the formation of shocks by the supersonic downflows. c) Enhanced field strengths were found at the tails of both, NEF- and CEF-carrying filaments. According with the induction equation, this can be a result of vertical advection processes caused by the supersonic downflows at the outer penumbra which can locally enhance the vertical field component at the tails of the NEF-carrying filaments. In the CEF-carrying filaments, the occurrence of converging downflows at the inner penumbra can lead to the intensification of the vertical fields, but there the close vicinity of the umbral field also plays an important role.

- The constant optical depth levels are strongly depressed in the sink locations due to the strong reduction of the density of the cooler downflowing gas. This causes that the enhanced fields at the filament tails look stronger along the $\tau=1$ level.

- Finally, in Chapter 5 we have tested the results of the SPINOR 2D inversions presented in Chapter 3 (applied to the Hinode SP scan from active region NOAA 10930) in a group of pixels located close to the inner boundary of the penumbral sector hosting the CEF. The peculiarity of these pixels lies in the unusually large penumbral magnetic field strengths (in excess of $7 \mathrm{kG}$ ) and supersonic LOS velocities returned by the inversions, as well as in the very complex spectral profile shapes of all four observed Stokes parameters from those pixels. In the following, I summarize our main findings:

- The observed Stokes profiles in the large field pixels (LFPs) display large asymmetries as well as seemingly very large wavelength shifts and Zeeman splittings, and their Stokes $V$ profiles contain more than two lobes.

- The best fits to the observed Stokes profiles obtained with the SPINOR 2D inversions are relatively poor in the LFPs, compared to the SPINOR 2D results in the bulk of the penumbra. Hence, the inverted parameters likely involve large errors in the estimated field values for the LFPs.

- The Zeeman direct splitting and center-of-gravity (COG) methods applied to the profiles in the LFPs provide very different results. Furthermore, none of them is entirely consistent in the two Fe I lines. The difference between COG and direct splitting methods are partly due to the non-longitudinal direction of the field. Their inconsistency for the two lines is possibly a result of large gradients in $B$ and $v_{L O S}$, which are not taken into account in these methods, but which might be responsible for the large asymmetries of the Stokes profiles.

- Method 1 (Zeeman direct splitting applied to the Stokes $V$ profiles of the LFPs) finds two different solutions ( $B \sim 3.5 \mathrm{kG}$ and $B \sim 9 \mathrm{kG}$ in line 1, and $B \sim 3.5 \mathrm{kG}$ and $B \sim 7 \mathrm{kG}$ in line 2). The solution chosen by the method 
depends on the shapes of the multi-lobed Stokes $V$ profiles, which in some of the LFPs display inner lobes whose signal surpasses that of the external lobes and hence, method 1 computes fields of the order of $3.5 \mathrm{kG}$. The second and largest solution computed with this method corresponds to LFPs whose Stokes $V$ profiles display the largest signals at their external lobes.

- SPINOR inversions considering two unresolved atmospheric components were tested in two of the LFPs and applied to their observed and deconvolved spectra. These inversions produced better fits but involved a larger number of free parameters than the single-component inversions, which significantly affects the reliability of their results. The resultant best-fits assign very large velocities to one atmosphere and nearly zero velocity to the other, and trade the extremely large field strengths for very large Doppler shifts. Nonetheless, even the two-component models suggest that the strongly redshifted atmospheric components in the LFPs contain fairly vertical fields, with the same polarity as the umbral field, which are unusually strong (5-6 kG) compared with typical penumbral field strenghts, as well as supersonic LOS flow speeds that can be interpreted as supersonic downflows.

- The quality of the best-fits resulting from all the inversions presented in Chapter 5 was tested by using the Bayesian Information Criterion (BIC), which equally penalizes the merit function of the fit, $\chi^{2}$, the number of free parameters in the model, $n$, and the number of observed points, $N$. The heightdependent model preferred by the BIC is SPINOR 2D.

The Stokes profiles observed in the LFPs are definitively unusual, and they probably contain the largest fields ever measured in penumbral environments. To estimate, with an appropriate accuracy, the actual magnetic field strengths in the LFPs will require more sophisticated analysis techniques (see, e.g., next Section).

Nonetheless, the picture emerging from the SPINOR 2D inversions is qualitatively similar to the flow-field configuration observed in the simulations as presented in Chapter 4, where a field intensification can occur at the sink locations of the CEFcarrying filaments at the inner penumbra (where the filament tails are located) due to the occurrence of converging supersonic downflows. In addition, the proximity of the umbral fields coupled with a lowered $\tau=1$ level due to a low density of the downflowing gas, leads to the exposure of stronger fields from deeper geometrical heights in the simulations. The similar characteristics observed in the spectra of the LFPs and in the synthetic spectra computed from the simulations, support the presence of intense penumbral fields in the LFPs. They also support the qualitative scenario provided by the SPINOR 2D inversions.

In the following section, we provide an outlook by highlighting some important aspects in our present study that need to be further investigated or continued in order to better understand the gas dynamics as well as the magnetic and thermal structures in sunspot penumbrae. 


\subsection{Outlook}

Sunspot penumbrae comprise a rich variety of phenomena which have prompted the continuous improvement of solar instrumental capabilities, as well as the implementation of new analysis techniques. Nonetheless, there are still many aspects of sunspot penumbrae that are not yet well understood, namely the formation processes, the establishment of an 'uncombed' magnetic configuration, the start of the NEF, its thermal structure, the chromospheric IEF, the occurrence of photospheric CEFs, and a long list of other aspects involving the interaction of the magnetic field with small- and large-scale flows.

In particular, the discovery of a new type of penumbral large-scale photospheric flows adds further difficulties to our conception of penumbral dynamics. Some open questions regarding the transitory CEFs, but also regarding the general properties of sunspots, can be listed as follows:

- Connection with chromospheric IEF. Although the IEF has not been analyzed in this thesis, the question of an IEF-CEF connection is compelling. The IEF is present in all sunspots observed in the chromosphere. It is also a robust feature in MHD simulations and its nature in terms of driving forces will eventually become accessible numerically. However, in the present simulations the IEF is possibly affected by the proximity of the closed top boundary of the simulation domain and by the artificially reduced Alfvén speed, as well as by numerical diffusion. There are also other shortcomings of the simulations: They are too small to cover also the outer footpoints of the IEF. Where do these lie? In addition, the restriction of the radiative energy transfer to LTE is also a limitation that could strongly influence the energetics and properties of the modelled IEF. Hence, an energetic analysis of the IEF and its connection with photospheric CEF can only be made when improved simulations become available. In addition, improvements in the simultaneous high-resolution observations of the two layers (photosphere and chromosphere) using state-of-theart two-dimensional spectropolarimeters will allow answering the question about the possible connection between IEF and the photospheric penumbral flows.

- Influence on the field topology in the higher layers of the atmosphere. The availability of Ca II H filtergrams from AR 10930 from the time of the AR's first appearance over the solar west limb on 2006-12-06 until the disappearance of the CEF on 2006-12-09 when the center-side penumbra shows the NEF only, provides us with the opportunity to investigate some physical properties in the low chromosphere overlying the penumbral sector hosting CEFs. A quick look into the temporal sequence of $\mathrm{Ca}$ II $\mathrm{H}$ filtergrams shows that the evolution of the anomalous part of the penumbra in the AR is associated with high chromospheric activity, which might be either associated to the formation of the anomalous part of the penumbra or a direct response to the photospheric CEFs. Therefore, to investigate the higher layers of the atmosphere in this AR will be part of a future work, and the result will hopefully help us understand the formation process of the anomalous penumbra, as well as the persistence of the CEF for $\sim 2$ days and the conditions leading to its reversal into a NEF.

- Occurrence frequency of CEFs. The appearance of intermittent CEFs in the sim- 
ulated penumbra analyzed in Chapter 4, with very short life-times, suggests that we should not discard the possibility of CEFs being a more common aspect of the penumbral dynamics. To thoroughly investigate the true occurrence frequency of CEFs, fast-cadence spectropolarimeters as well as realistic MHD numerical simulations of sunspot penumbrae are necessary.

- Reality of superstrong penumbral magnetic fields. Observations in a different spectral region, sampling the photosphere in deeper layers, bear a big potential to answer the question about the reality of the high magnetic field strengths. The infrared lines around $1.56 \mu \mathrm{m}$ are formed about 50-100 km deeper than the Fe I lines observed with Hinode SOT/SP. Only very recently have such measurements become available at the required spatial resolution, thanks to new instrument developments at MPS. The extremely high magnetic sensitivity $\left(g_{\text {eff }}=3\right)$ of the Fe $15648 \AA$ line will potentially allow for the unambiguous detection of such high magnetic field values, if they actually exist.

- Implications of the existence of superstrong magnetic fields in sunspot penumbrae. An important aspect to consider in the presence of field strengths in excess of $7 \mathrm{kG}$ is if the Paschen-Back effect plays a role on the splitting of the photospheric spectral lines. This is of particular importance for the pair of Fe I lines at $\lambda=6301.5$ and $6302.5 \AA$ whose small wavelength separation makes them susceptible to the incomplete Paschen-Back effect in the presence of field strengths of the order of 10 $\mathrm{kG}$. As an allusion to stellar contexts, many difficulties have been encountered when trying to model the highly asymmetric and wavelength shifted observed spectra from strongly magnetized Ap stars with synthetic spectra within the Zeeman regime (Mathys 1990). Therefore, it is of great importance to investigate how the best-fits to the observed spectra would be affected if treated in the Paschen-Back regime. Although the so-called ghost components (appearance of extra components which are forbidden in the absence of a magnetic field and have negligible strengths in the Zeeman effect regime since they violate the selection rules on the total angular momentum quantum number $J$, but can lead to an increase in the number of lines as well as in the number of magnetic components for certain multiplets within the Paschen-Back regime (e.g., Stift et al. 2008)) are likely unobservable, there could be other problems when inversions of lines in the Paschen-Back regime are treated in the Zeeman approximation. This investigation will be carried out in a future work.

- Exploring new techniques to determine the physical properties of sunspots in a geometrical height scale. The inversion codes typically provide the physical parameters as functions of the optical depth. This represents a major shortcoming for the computation of horizontal variations of the atmospheric parameter and thus, it limits our understanding of the nature of various observable phenomena (e.g. NEF and CEF as studied in Chapter 3). Several methods aimed to extract the true geometrical height scale from the observations have been developed (e.g. Mathew et al. 2004, Carroll and Kopf 2008, Puschmann et al. 2010, cf. Maltby (1997), Solanki et al. (1993), Martínez Pillet and Vazquez (1993)). However, all those methods are based on assumptions that can lead to large uncertainties in the computed geomet- 
rical heights. Therefore, an important step in solar physics will be the development of new inversion techniques aimed at inferring the atmospheric physical conditions on geometrical height scales with sufficient accuracy. The recently implemented MHD-Assisted Stokes Inversion (MASI; Riethmüller et al. 2017) is a still developing, but highly promising technique inspired by the idea of a full spatially and temporally coupled inversion that is able to integrate MHD simulations into the Stokes inversions of a time series. MASI aims at providing the stratifications of the atmospheric parameters on a geometrical height scale, as well as at giving insight into all physical quantities allowed by the MHD data (e.g. horizontal velocities, density, pressure, horizontal force balance, etc.) which are otherwise not available in traditional inversions since they are not included in the spectral lines. Hence, it would be worthwhile to address the optimization of this technique as well as to consider its further improvements in the near future.

- The formation of sunspot penumbrae. To understand the initiation of the Evershed flow, it is essential to first understand how penumbrae are formed. However, the formation of sunspot penumbrae has been observed only on a few occasions, and its simulation involves challenging difficulties. Consequently, the nature of the penumbra is still poorly known and it remains an open question. Simultaneous observations of the magnetic field vector in the photosphere and chromosphere are necessary in order to investigate where the main driving mechanisms for penumbrae formation occur in the atmosphere. Likewise, high-temporal-cadence spectropolarimetric observations can substantially help to capture the essential fingerprints of a forming penumbra during its early stages.

- Evolution of sunspot penumbrae. Another interesting question about sunspot penumbrae concerns their temporal evolution. Little is known about the changes on their fine structure during a sunspot's life, for instance, if the penumbral filaments display physical properties that depend on the sunspot's age or evolutionary stage, and the implications of those possible changes for sunspot decay. A statistical analysis of young, medium-age, and long-lived sunspots might help identify typical trends on the evolution of the magnetic, thermal and flow structures in sunspots.

- Dependence of the sunspot fine structure on the sunspot area. Also, the question about how the fine structure of umbrae and penumbrae relates to sunspot size is compelling. In particular, Is the Evershed flow different in larger or smaller sunspots? Does the umbral brightness or the number of umbral dots depend on the sunspot size? Answering these questions is relevant for constructing a comprehensive sunspot model or to distinguish between the current models, as well as for testing the validity of helioseismic inversions. Furthermore, learning how the sunspot spatial scales affect the variety of physical effects observed on the solar surface (e.g. those due to magneto-convection), will help to extrapolate our knowledge about sunspots to the stellar cases (i.e. starspots; see below).

- Connection between sunspots and starspots. Starspots can, naturally, have very distinct properties to sunspots, e.g. different typical sizes, lifetimes, magnetic field strengths, brightness, latitudinal appearance, evolution, etc, depending on the properties of the star. Nonetheless, for stars with convective envelopes the fundamental 
physical processes regarding the surface magneto-convective effects must be similar to those occurring in the Sun. Therefore, an important question to address regarding the stellar surface magnetism is if the starspots' global structure is similar to that of sunspots, for instance if starspots comprise well defined umbral and penumbral magnetic field regions as well as the associated characteristic flows observed in sunspots (e.g. the Evershed flow). Unfortunately, the stellar observational capabilities are still far from reaching the necessary spatial resolutions to address this question. Alternatively, the properties of starspots can be explored by means of MHD simulations, extending those of sunspots to other stars with outer convection zones. An important step within the numerical approach, towards a more detailed understanding of the sunspots-starspots connection, would be the investigation of starspots' fine and global structures as well as of their characteristic flow dynamics in differently active stars by means of realistic MHD simulations. The results of such an investigation might also provide a basis to determine how the starspot's surface structure influences the disk-integrated polarization signals in the stellar observations, as well as its implications on methods such as Zeeman Doppler imaging (ZDI). 



\section{Bibliography}

Anders, E., Grevesse, N., 1989, Abundances of the elements - Meteoritic and solar, Geochimica et Cosmochimica Acta, 53, 197

Asensio Ramos, A., Manso Sainz, R., Martínez González, M. J., Viticchié, B., Orozco Suárez, D., Socas-Navarro, H., 2012, Model Selection for Spectropolarimetric Inversions, ApJ, 748, 83

Baym, G., 1969, Lectures on Quantum Mechanics, Inc, New York

Beckers, J. M., 1969a, The profiles of Frauenhofer lines in the presence of Zeeman splitting - I: The Zeeman Triplet, Sol. Phys., 9, 372

Beckers, J. M., 1969b, The profiles of Frauenhofer lines in the presence of Zeeman splitting - II: Zeeman splitting for Dipole and Quadrupol Radiation, Sol. Phys., 10, 262

Beckers, J. M., Schröter, E. H., 1969, The Intensity, Velocity and Magnetic Structure of a Sunspot Region. IV: Properties of a Unipolar Sunspot, Sol. Phys., 10, 384-403

Bello González, N., Okunev, O. V., Domínguez Cerdeña, I., Kneer, S., Puschmann, K. G., 2005, Polarimetry of sunspot penumbrae with high spatial resolution, A\&A, 434, 317

Bellot Rubio, L. R., 1998, Structure of solar magnetic elements from the inversion of Stokes spectra, Ph.D. thesis, Instituto de Astrofísica de Canarias, Spain, Spain

Bellot Rubio, L. R., 2010, The Evershed Flow and the Brightness of the Penumbra, pp. 193-209, Springer, Springer-Verlag Berlin Heidelberg

Bellot Rubio, L. R., Ruiz Cobo, B., Collados, M., 1998, An Hermitian Method for the Solution of Polarized Radiative Transfer Problems, ApJ, 506, 805

Bellot Rubio, L. R., Balthasar, H., Collados, M., Schlichenmaier, R., 2003, Field-aligned Evershed flows in the photosphere of a sunspot penumbra, A\&A, 403, L47

Bellot Rubio, L. R., Balthasar, H., Collados, M., 2004, Two magnetic components in sunspot penumbrae, A\&A, 427, 319-344

Bellot Rubio, L. R., Langhans, K., Schlichenmaier, R., 2005, Multi-line spectroscopy of dark-cored penumbral filaments, A\&A, 443, L7 
Bellot Rubio, L. R., Tsuneta, S., Ichimoto, K., Katsukawa, Y., Lites, B. W., Nagata, S., Shimizu, T., Shine, R. A., Suematsu, Y., Tarbell, T. D., Title, A. M., del Toro Iniesta, J. C., 2007, Vector Spectropolarimetry of Dark-cored Penumbral Filaments with Hinode, ApJ, 668, L91

Bellot Rubio, L. R., Trischler, A., Martínez Pillet, V., 2008, Spectropolarimetry of a decaying sunspot penumbra, ApJ, 676, 689-703

Benevolenskaya, E. E., Hoeksema, J. T., Kosovichev, A. G., Scherrer, P. H., 1999, The Interaction of New and Old Magnetic Fluxes at the Beginning of Solar Cycle 23, ApJ, 517, L163

Bharti, L., Solanki, S. K., Hirzberger, J., 2010, Evidence for Convection in Sunspot Penumbrae, ApJ, 722, L194

Biermann, L., 1941, Der gegenwärtige Stand der Theorie konvektiver Sonnenmodelle, Vierteljahrsschrift der Astronomischen Gesellschaft, 76, 194-200

Boris, J. P., 1970, NRL Memorandum Report, 2167

Börner, P., Kneer, F., 1992, High resolution observations of the Evershed flow, A\&A, 259, 307-312

Borrero, J. M., 2007, The structure of sunspot penumbrae. IV. MHS equilibrium for penumbral flux tubes and the origin of dark core penumbral filaments and penumbral grains, A\&A, 471, 967

Borrero, J. M., 2009, Models and observations of sunspot penumbrae, ScChG: Physics, Mechanics and Astronomy, 52, 1670-1677

Borrero, J. M., Bellot Rubio, L. R., 2002, A two-component model of the solar photosphere from the inversion of spectral lines, A\&A, 385, 1056-1072

Borrero, J. M., Ichimoto, K., 2011, Magnetic Structure of Sunspots, Living Rev. Solar Phys., 8, 98

Borrero, J. M., Solanki, S. K., 2008, Are There Field-Free Gaps near $\tau=1$ in Sunspot Penumbrae?, ApJ, 687, 668-677

Borrero, J. M., Solanki, S. K., Bellot Rubio, L. R., Lagg, A., Mathew, S. K., 2004, Thermal-magnetic relation in a sunspot and a map of its Wilson depression, A\&A, 422,1093

Borrero, J. M., Lagg, A., Solanki, S. K., Collados, M., 2005, On the fine structure of sunspot penumbrae. II. The nature of the Evershed flow, A\&A, 436, 333-345

Borrero, J. M., Solanki, S. K., Lagg, A., Socas-Navarro, H., Lites, B., 2006, On the fine structure of sunspot penumbrae. III. The vertical extension of penumbral filaments, A\&A, 450, 383 
Borrero, J. M., Tomczyk, S., Kubo, M., Socas-Navarro, H., Schou, J., Couvidat, S., Bogart, R., 2011, VFISV: Very Fast Inversion of the Stokes Vector for the Helioseismic and Magnetic Imager, Sol. Phys., 273, 267-293

Botha, G. J. J., Rucklidge, A. M., Hurlnurt, N. E., 2011, Nonlinear Three-dimensional Magnetoconvection around Magnetic Flux Tubes, ApJ, 731, 108

Brandenburg, A., 2005, The case for a distributed solar dynamo shaped by the nearsurface shear, ApJ, 625

Brandenburg, A., Rogachevskii, I., Kleeorin, N., 2016, Magnetic concentrations in stratified turbulence: the negative effective magnetic pressure instability, New Journal of Physics, 18, 12

Brault, J. W., 1978, Future Solar Optical Observations. Needs and Constraints, Proc. JOSO Workshop, 106, 33

Bray, R. J., 1964, Sunspots, Chapman and Hall

Brown, T. M., Christensen-Dalsgaard, J., Dziembowsky, W. A., Goode, P., Gough, D. O., Morrow, C. A., 1989, Inferring the sun's internal angular velocity from observed pmode frequency splittings, ApJ, 343, 526

Bruls, J. H. M. J., Solanki, S. K., Carlsson, M., Rutten, R. J., 1995, Infrared lines as probes of solar magnetic features. VIII. MgI $12 \mu \mathrm{m}$ diagnostics of sunspots, A\&A, 293, 225

Bruls, J. H. M. J., Vollmöller, P., Schüssler, M., 1999, Computing radiative heating on unstructured spatial grids, A\&A, 348, 233-248

Brummell, N. H., Tobias, S. M., Thomas, J. H., Weiss, N. O., 2008, Flux Pumping and Magnetic Fields in the Outer Penumbra of a Sunspot, ApJ, 686, 1454

Brun, A. S., Browning, M. K., Dikpati, M., Hotta, H., Strugarek, A., 2015, Recent Advances on Solar Global Magnetism and Variability, Space Sci. Rev., 196, 101

Cabrera Solana, D., Bellot Rubio, L. R., del Toro Iniesta, J. C., 2005, Sensitivity of spectral lines to temperature, velocity, and magnetic field, A\&A, 439, 687-699

Cap, F., 1994, Lehrbuch der Plasmaphysik und Magnetohydrodynamik (Textbook of plasma physics and magneto-hydrodynamics), Springer-Verlag, Wien

Carroll, T. A., Kopf, M., 2008, Zeeman-tomography of the solar photosphere. Threedimensional surface structures retrieved from Hinode observations, A\&A, 481, L37-L40

Cauzzi, G., Smaldone, L. A., Balasubramaniam, K. S., Keil, S. L., 1993, On the calibration of line-of-sight magnetograms, Sol. Phys., 146, 207-227

Chandrasekhar, S., 1961, Clarendon Press, Oxford

Charbonneau, P., 2005, Dynamo Models of the Solar Cycle, Living Rev. Solar Phys., 2, 2 
Charbonneau, P., 2010, Dynamo Models of the Solar Cycle, Living Rev. Solar Phys., 7, 3

Charbonneau, P., 2014, Solar Dynamo Theory, ARA\&A, 52, 251

Charbonneau, P., Christensen-Dalsgaard, J., Henning, R., Larsen, R. M., Schou, J., Thompson, M. J., Thomczyk, S., 1999, Helioseismic Constraints on the Structure of the Solar Tachocline, ApJ, 527, 445

Chen, F., Rempel, M., Fan, Y., 2017, Emergence of Magnetic Flux Generated in a Solar Convective Dynamo. I. The Formation of Sunspots and Active Regions, and The Origin of Their Asymmetries, ApJ, 846, 149

Cheung, M. C., Rempel, M., Title, A. M., Schüssler, M., 2010, Simulation of the Formation of a Solar Active Region, ApJ, 720, 233

Choudhuri, A. R., 1986, The dynamics of magnetically trapped fluids. I. Implications for umbral dots and penumbral grains, ApJ, 302, 809-825

Choudhuri, A. R., 1990, On the possibility of an alpha-sq omega-type dynamo in a thin layer inside the sun, ApJ, 355, 733

Choudhuri, A. R., 1998, The physics of fluids and plasmas, Cambridge University Press, New York

Clyne, J., Rast, M., 2005, Proc. SPIE, 5669, 284

Clyne, J., Mininni, P., Norton, A., Rast, M., 2007, New J. Phys., 9, 301

Collados, M., Martínez Pillet, V., Ruiz Cobo, B., del Toro Iniesta, J. C., Vazquez, M., 1994, Observed differences between large and small sunspots, A\&A, 291, 622

Cowling, C. T., 1957, Magnetohydrodynamics, Interscience, New York

Cowling, T. G., 1953, The Sun, chap. Solar Electrodynamics, p. 532, The University of Chicago Press, Chicago

Culhane, J. L., Doschek, G. A., Watanabe, T., Smith, A., Brown, C., Hara, H., Harra, L. K., James, A. M., al Janabi, K., Kent, B., Korendyke, C., Lang, J., Mariska, J., Myers, A., Seely, J., Simnett, G., Tandy, J., Thomas, R., Windt, D. L., 2006, The extreme UV imaging spectrometer for the JAXA Solar-B mission, Proceedings of the SPIE, 6266, 62 660T

Danielson, R. E., 1961, The Structure of Sunspot Penumbras. II. Theoretical, ApJ, 134, 289

Danilovic, S., Gandorfer, A., Lagg, A., Schüssler, M., Solanki, S. K., Vögler, A., Katsukawa, Y., Tsuneta, S., 2008, The intensity contrast of solar granulation: comparing Hinode SP results with MHD simulations, A\&A, 484, L17-L20

Danilovic, S., Shüssler, M., Solanki, S. K., 2010, Magnetic field intensification: comparison of 3D MHD simulations with Hinode/SP results, A\&A, 509, A76 
Degenhardt, D., 1989, Stationary siphon flows in thin magnetic flux tubes, A\&A, 222, 297

Degenhardt, D., Wiehr, E., 1991, Spatial variation of the magnetic field inclination in a sunspot penumbra, A\&A, 252, 821

del Toro Iniesta, J. C., 2003, Introduction to Spectropolarimetry, Cambridge University Press, UK

del Toro Iniesta, J. C., Bellot Rubio, L. R., Collados, M., 2001, Cold, Supersonic Evershed Downflows in a Sunspot, ApJ, 549, L139

Denker, C., 1998, Speckle Masking Imaging of Sunspots and Pores, Sol. Phys., 180, 81

Dialetis, D., Mein, P., Alissandrakis, C. E., 1985, The Evershed flow as a steady-state homogeneous phenomenon, A\&A, 147, 93

Dikpati, M., Gilman, P. A., 2001, Flux-transport dynamos with $\alpha$-effect from global instability of tachocline differential rotation: A solution for magnetic parity selection in the sun, ApJ, 559, 428-442

Dikpati, M., Corbard, T., Thompson, M. J., Gilman, P. A., 2002, Flux Transport Solar Dynamos with Near-Surface Radial Shear, ApJ, 575, L41

Dunn, R. B., Zirker, J. B., 1973, The Solar Filigree, Sol. Phys., 33, 281

Esteban Pozuelo, S., Bellot Rubio, L. R., de la Cruz Rodríguez, J., 2015, Lateral downflows in sunspot penumbral filaments and their temporal evolution, ApJ, 803, 93

Evershed, J., 1909, Radial movement in sun-spots, MNRAS, 69, 454

Frisch, I. E., 1963, Optical Spectra of Atoms, Fizmatgiz, Moscow, Leningrad

Frutiger, C., 2000, Inversions of Zeeman Split Stokes Profiles: Application to solar and stellar surface structures, Ph.D. thesis, Institute of Astronomy, ETH Zürich, Switzerland, no. 13896

Frutiger, C., Solanki, S. K., 2001, Empirical models of solar magnetic flux-tubes and their non-magnetic surroundings, A\&A, 369, 646

Frutiger, C., Solanki, S. K., Fligge, M., Bruls, J. H. M. J., 2000, Properties of the solar granulation obtained from the inversion of low spatial resolution spectra, A\&A, 358, $1109-1121$

Frutiger, C., Solanki, S. K., Mathys, G., 2005, Fundamental parameters and granulation properties of Alpha Centauri A and B obtained from inversions of their spectra, A\&A, 444,549

Gandorfer, A. M., Povel, H. P., 1997, First observations with a new imaging polarimeter, A\&A, 328, 381 
Georgoulis, M. K., 2005, A New Technique for a Routine Azimuth Disambiguation of Solar Vector Magnetograms, ApJ, 629, L69

Giovanelli, R. G., Jones, H. P., 1982, The three-dimensional structure of atmospheric magnetic fields in two active regions, Sol. Phys., 79, 267-278

Gizon, L., Birch, A. C., 2005, Local Helioseismology, Living Rev. Solar Phys., 2, 5-41

Gizon, L., Birch, A. C., Spruit, H. C., 2010, Local Helioseismology: Three-Dimensional Imaging of the Solar Interior, ARA\&A, 48, 289-338

Gokhale, M. H., Zwaan, C., 1972, The Structure of Sunspots. I: Observational Constraints: Current Sheet Models, Sol. Phys., 26, 52

Golub, L., Rosner, R., Vaiana, G. S., Weiss, N. O., 1981, Solar magnetic fields - The generation of emerging flux, ApJ, 243, 309

Golub, L., DeLuca, E., Austin, G., Bookbinder, J., Caldwell, D., Cheimets, P., Cirtain, J., Cosmo, M., Reid, P., Sette, A., Weber, M., Sakao, T., Kano, R., Shibasaki, K., Hara, H., Tsuneta, S., Kumagai, K., Tamura, T., Shimojo, M., McCracken, J., Carpenter, J., Haight, H., Siler, R., Wright, E., Tucker, J., Rutledge, H., Barbera, M., Peres, G., Varisco, S., 2007, The X-Ray Telescope (XRT) for the Hinode Mission, Sol. Phys., 243,63

Grossmann-Doerth, U., Keller, C. U., Schüssler, M., 1996, Observations of the quiet Sun's magnetic field, A\&A, 315, 610

Gustafsson, B., 1973, Uppsala Astron. Obs. Ann., 5, 6

Hale, G. E., 1908, On the probable existence of a magnetic field in Sunspots, ApJ, 28, 315

Harvey, J. W., 1969, Magnetic Fields Associated with Solar Active-Region Prominences, Ph.D. thesis, University of Colorado, USA, NCAR cooperative thesis No. 17

Harvey, K. L., 1993, Magnetic Bipoles on the Sun, Ph.D. thesis, University of Utrecht, Netherlands

Harvey, K. L., Harvey, J. W., 1973, Observations of Moving Magnetic Features near Sunspots, Sol. Phys., 28, 61

Hathaway, D. H., 2015, The Solar Cycle, Living Rev. Solar Phys., 12, 87

Heinemann, T., Nordlund, A., Scharmer, G. B., Spruit, H. C., 2007, MHD Simulations of Penumbra Fine Structure, ApJ, 669, 1390-1394

Herzberg, G., 1945, Atomic spectra and atomic structure, Dover, New York

Hirzberger, J., Bonet, J. A., Sobotka, M., Vázquez, M., Hanslmeier, A., 2002, Fine structure and dynamics in a light bridge inside a solar pore, A\&A, 383, 275 
Holmes, J., 1963, Micrometer and photometric observations of a sunspot velocity field, MNRAS, 126, 155

Howe, R., Christensen-Dalsgaard, J., Hill, F., Komm, R. W., Larsen, R. M., Schou, J., Thompson, M. J., Toomre, J., 2000, Dynamic Variations at the Base of the Solar Convection Zone, Science., 287, 2456

Ichimoto, K., Shine, R. A., Lites, B., Kubo, M., Shimizu, T., Suematsu, Y., Tsuneta, S., Katsukawa, Y., Tarbell, T. D., Title, A. M., Nagata, S., Yokoyama, T., Shimojo, M., 2007, Fine-Scale Structures of the Evershed Effect Observed by the Solar Optical Telescope aboard Hinode, PASJ, 59, 593

Ichimoto, K., Lites, B., Elmore, D., Suematsu, Y., Tsuneta, S., Katsukawa, Y., Shimizu, T., Shine, R., Tarbell, T., Title, A., Kiyohara, J., Shinoda, K., Card, G., Lecinski, A., Streander, K., Nakagiri, M., Miyashita, M., Noguchi, M., Hoffmann, C., Cruz, T., 2008, Polarization Calibration of the Solar Optical Telescope onboard Hinode, Sol. Phys., 249, 233

Jahn, K., Schmidt, H. U., 1994, Thick penumbra in a magnetostatic sunspot model, A\&A, 290, 295-317

Joshi, J., Lagg, A., Hirzberger, J., Solanki, S. K., 2017a, Three-dimensional magnetic structure of a sunspot: Comparison of the photosphere and upper chromosphere, A\&A, 604, A98

Joshi, J., Lagg, A., Hirzberger, J., Solanki, S. K., Tiwari, S. K., 2017b, Vertical magnetic field gradient in the photospheric layers of sunspots, A\&A, 599, A35

Jurčak, J., Bello González, N., Schlichenmaier, R., Rezaei, R., 2015, A distinct magnetic property of the inner penumbral boundary. Formation of a stable umbra-penumbra boundary in a sunspot, A\&A, 580, L1

Jurčak, J., Bello González, N., Schlichenmaier, R., Rezaei, R., 2017, A distinct magnetic property of the inner penumbral boundary. ii. formation of a penumbra at the expense of a pore, $A \& A, 597, A 60$

Jǔrcák, J., 2011, Azimuthal variations of magnetic field strength and inclination on penumbral boundaries, A\&A, 531, A118

Jǔrcák, J., Martínez Pillet, V., Sobotka, M., 2006, The magnetic canopy above light bridges, A\&A, 453, 1079-1088

Jǔrcák, J., Bellot Rubio, L. R., Sobotka, M., 2014, Orphan penumbrae: Submerging horizontal fields, A\&A, 564, A91

Keppens, R., Martínez Pillet, V., 1996, The magnetic structure of pores and sunspots derived from Advanced Stokes Polarimeter data, A\&A, 316, 229

Khomenko, E., Collados, M., 2015, Oscillations and Waves in Sunspots, Living Rev. Solar Phys., 12, 6 
Kiess, C., Rezaei, R., Schmidt, W., 2014, Properties of sunspot umbrae observed in cycle 24, A\&A, 565, A52

Kitiashvili, I. N., Kosovichev, A. G., Wray, A. A., Mansour, N. N., 2009, Traveling Waves of Magnetoconvection and the Origin of the Evershed Effect in Sunspots, ApJ, 700, L178-L181

Kleint, L., Sainz Dalda, A., 2013, Unusual Filaments inside the Umbra, ApJ, 770, 74

Klimchuck, J. A., 2006, On Solving the Coronal Heating Problem, Sol. Phys., 234, 41-77

Kopp, G., Rabin, D., 1992, Sol. Phys., 141, 253

Kosugi, T., Matsuzaki, K., Sakao, T., Shimizu, T., Sone, Y., Tachikawa, S., Hashimoto, T., Minesugi, K., Ohnishi, A., Yamada, T., Tsuneta, S., Hara, H., Ichimoto, K., Suematsu, Y., Shimojo, M., Watanabe, T., Shimada, S., Davis, J. M., Hill, L. D., Owens, J. K., Title, A. M., Culhane, J. L., Harra, L. K., Doschek, G. A., Golub, L., 2007, The Hinode (Solar-B) Mission: An Overview, Sol. Phys., 243, 3

Kuckein, C., Martínez Pillet, V., Centeno, R., 2012, An active region filament studied simultaneously in the chromosphere and photosphere. I. Magnetic structure, A\&A, 539, A131

Lagg, A., Woch, J., Kripp, N., Solanki, S. K., 2004, Retrieval of the full magnetic vector with the He I multiplet at $1083 \mathrm{~nm}$. Maps of an emerging flux region, A\&A, 414, $1109-1120$

Lagg, A., Ishikawa, R., Merenda, L., Wiegelmann, T., Tsuneta, S., Solanki, S. K., 2009, Internetwork Horizontal Magnetic Fields in the Quiet Sun Chromosphere: Results from a Joint Hinode/VTT Study, Astronomical Society of the Pacific Conference Series, 415, 327

Lagg, A., Solanki, S. K., van Noort, M., Danilovic, S., 2014, Vigorous convection in a sunspot granular light bridge, A\&A, 568, A60

Lagg, A., Lites, B., Harvey, J., Gosain, S., Centeno, R., 2017, Measurements of Photospheric and Chromospheric Magnetic Fields, Space Sci. Rev., 210, 37

Landi Degl'innocenti, E., 1983, Polarization in spectral lines. I - A unifying theoretical approach. II - A classification scheme for solar observations, Sol. Phys., 85, 3

Landi Degl'innocenti, E., Landolfi, M., 2004, Polarization in spectral lines, Kluwer Academic Publishers, United States of America

Langhans, K., Scharmer, G. B., Kiselman, D., Löfdahl, M. G., 2007, Observations of dark-cored filaments in sunspot penumbrae, A\&A, 464, 763

Leka, K. D., Skumanich, A., 1998, The evolution of pores and the development of penumbrae, ApJ, 507, 454 
Lites, B., Casini, R., Garcia, J., Socas-Navarro, H., 2007, Mem. Soc. Astron. Italiana, 78, 148

Lites, B. W., 2007, Summary Review: Science with the SOT, in New Solar Physics with Solar-B Mission ASP Conf. Ser., (Eds.) K. Shibata, S. Nagata, T. Sakurai, vol. 369, p. 579

Lites, B. W., Ichimoto, K., 2013, The SP_PREP Data Preparation Package for the Hinode Spectro-Polarimeter, Sol. Phys., 283, 601

Lites, B. W., Skumanich, A., Scharmer, G. B., 1990, High-resolution spectra of solar magnetic features. I - Analysis of penumbral fine structure, ApJ, 355, 329

Lites, B. W., Dunn, R. B., Elmore, D. F., Tomczyk, S., Skumanich, A., Streander, K. V., 1992, First Results from the Advanced Stokes Polarimeter, American Astronomical Society Meeting, 180, 1201

Lites, B. W., Elmore, D. F., Seagraves, P., Skumanich, A. P., 1993, Stokes Profile Analysis and Vector Magnetic Fields. VI. Fine Scale Structure of a Sunspot, ApJ, 418, 928

Lites, B. W., Elmore, D. F., Streander, K. V., 2001, The Solar-B Spectro-Polarimeter, in Advanced Solar Polarimetry - Theory, Observation, and Instrumentation - ASP Conference Proceedings, (Ed.) M. Sigwarth, vol. 236, p. 33

Lites, B. W., Socas Navarro, H., Skimanich, A., Shimizu, T., 2002, Converging flows in the penumbra of a $\delta$ sunspot, ApJ, 575, 1131-1143

Lites, B. W., Akin, D. L., Card, G., Card, G., Cruz, T., Duncan, D. W., Edwards, C. G., Elmore, D. F., Hoffmann, C., Katsukawa, Y., Katz, N., Kubo, M., Ichimoto, K., Shimizu, T., Shine, R. A., Streander, K. V., Suematsu, A., Tarbell, T. D., Title, A. M., Tsuneta, S., 2013, The Hinode Spectro-Polarimeter, Sol. Phys., 283, 579

Livingston, W., 2002, Sunspots Observed to Physically Weaken in 2000-2001, Sol. Phys., 207,41

Livingston, W., Harvey, J., 1971, The Kitt Peak Magnetograph. Iv: 40-CHANNEL Probe and the Detection of Weak Photospheric Fields, Solar Magnetic Fields, 43, 51

Livingston, W., Harvey, J. W., 2006, Sunspots with the Strongest Magnetic Fields, Sol. Phys., 239, 41

Loughhead, R. E., 1968, High-Resolution Photography of the Solar Chromosphere, Sol. Phys., 5, 489

Louis, R. E., Beck, C., Mathew, S. K., Venkatakrishnan, P., 2014, Anomalous flows in a sunspot penumbra, A\&A, 570, A92

Lozitsky, V. G., 1993, The problem of superstrong magnetic fields in the solar atmosphere, Kinematika i Fizika Nebesnykh Tel., 9, 23 
Lucy, L. B., 1974, An iterative technique for the rectification of observed distributions, AJ, 79, 745

Maltby, P., 1964, On the velocity field in sunspots, ApNr, 8, 205

Maltby, P., 1997, Advances in Physics of Sunspot, in 1st Advances in Solar Physics Euroconference, vol. 118, p. 91, ASP Conf. Ser.

Mariani, F., Villante, U., Bruno, R., Bavassano, B., Ness, N. F., 1979, An extended investigation of Helios 1 and 2 observations: the interplanetary magnetic field between 0.3 and 1 AU, Sol .Phys., 63, 411

Martínez Pillet, V., 2000, Spectral signature of uncombed penumbral magnetic fields, A\&A, 361, 734-742

Martínez Pillet, V., 2002, Decay of sunspots, Astronom. Nach., 323, 342

Martínez Pillet, V., Vazquez, M., 1993, The continuum intensity-magnetic field relation in sunspot umbrae, A\&A, 270, 494-508

Mason, J., Hughes, D., Thobias, S. M., 2002, The Competition in the Solar Dynamo between Surface and Deep-seated $\alpha$-Effects, ApJ, 580, L89

Mathew, S. K., Lagg, A., Solanki, S. K., Collados, M., Borrero, J. M., Berdyugina, S., Krupp, N., Woch, J., Frutiger, C., 2003, Three dimensional structure of a regular sunspot from the inversion of IR Stokes profiles, A\&A, 410, 695

Mathew, S. K., Solanki, S. K., Lagg, A., Collados, M., Borrero, J. M., Berdyugina, S., 2004, Thermal-magnetic relation in a sunspot and amap of its Wilson depression, A\&A, 422,693

Mathys, G., 1990, AP stars with resolved Zeeman split lines, A\&A, 232, 151

McIntosh, P. S., 1981, The Physics of Sunspots, Sunspots, NSO, p. 7

Metcalf, T. R., Leka, K. D., Lites, B. W., Georgoulis, M. K., Pevtsov, A. A., Balasubramaniam, K. S., Gary, G. A., Jing, J., Li, J., Liu, Y., Wang, H. N., Abramenko, V., Yurchyshyn, V., Moon, Y. J., 2006, An overview of existing algorithms for resolving the $180^{\circ}$ ambiguity in vector magnetic fields: Quantitative tests with synthetic data, Sol. Phys., 237, 267-296

Meyer, F., Schmidt, H. U., 1968a, A Model for the Evershed Flow in Sunspots, MitAG, 25,194

Meyer, F., Schmidt, H. U., 1968b, Magnetisch ausgerichtete Strömungen zwischen Sonnenflecken, Zeitschrift Angewandte Mathematik und Mechanik, 48, 218

Meyer, F., Schmidt, H. U., Weiss, N. O., Wilson, P. R., 1974, The growth and decay of sunspots, MNRAS, 169, 35-37

Meyer, F., Schmidt, H. U., Weiss, N. O., 1977, The stability of sunspots, MNRAS, 179, 741-761 
Miesch, M. S., 2005, Large-scale dynamics of the convection zone and tachocline, Living Rev. Solar Phys., 2, 139

Montesinos, B., Thomas, J. H., 1997, The Evershed effect in sunspots as a siphon flow along a magnetic flux tube, Nature, 390, 485-487

Moore, C. E., 1945, A Multiplet Table of Astrophysical Interest, Princeton University Observatory, Princeton, New Jersey

Moore, R. L., 1981, Dynamic phenomena in the visible layers of sunspots, Space Sci. Rev., 28, 387

Moradi, H., Baldner, C., Birch, A. C., Braun, D. C., Cameron, R. H., Duvall, T. L., Gizon, L., Haber, D., Hanasoge, S. M., Hindman, B. W., Jackiewicz, J., Khomenko, E., Komm, R., Rajaguru, P., Rempel, M., Roth, M., Schlichenmaier, R., Schunker, H., Spruit, H. C., Strassmeier, K. G., Thompson, M. J., Zharkov, S., 2010, Modeling the subsurface structure of sunspots, Sol. Phys., 267, 1-62

Muller, R., 1976, Characteristics of the Displacement of the Penumbral Bright Grains of Sunspots, Sol. Phys., 48, 101

Murabito, M., Romano, P., Guglielmino, S. L., Zuccarello, F., Solanki, S. K., 2016, Formation of the penumbra and start of the Evershed flow, ApJ, 825, 75

Parker, E. N., 1958, Dynamics of the interplanetary gas and magnetic fields, ApJ, 128, 664

Parker, E. N., 1963, Kinematical Hydromagnetic Theory and its Application to the Low Solar Photosphere, ApJ, 138, 552

Parker, E. N., 1975, The Nature of the Sunspot Phenomenon. IV: The Intrinsic Instability of the Magnetic Configuration, Sol. Phys., 40, 291

Parker, E. N., 1978, Hydraulic concentration of magnetic fields in the solar photosphere. VI - Adiabatic cooling and concentration in downdrafts, ApJ, 221, 368-377

Parker, E. N., 1979, Sunspots and the physics of magnetic flux tubes. IX. Umbral dots and longitudinal overstability, ApJ, 234, 333-347

Parker, E. N., 1994, Theoretical properties of Omega-loops in the convective zone of the Sun. 1: Emerging bipolar magnetic regions, ApJ, 433, 867-874

Petrovay, K., Moreno-Insertis, F., 1997, Turbulent Erosion of Magnetic Flux Tubes, ApJ, 485,398

Pizzo, V. J., 1986, Numerical solution of the magnetostatic equations for thick flux tubes, with application to sunspots, pores, and related structures, ApJ, 302, 785-808

Pizzo, V. J., 1990, Numerical modeling of solar magnetostatic structures bounded by current sheets, ApJ, 365, 764-777 
Povel, H., 1995, Imaging Stokes polarimetry with piezoelastic modulators and chargecoupled-device image sensors, Optical Engineering, 34, 1870

Press, W. H., Teukolsky, S. A., Vetterling, W. T., Flannery, B. P., 1992, Cambridge University Press, New York

Press, W. H., Teukolsky, S. A., Vetterling, W. T., Flannery, B. P., 2007, Numerical Recipes: The Art of Scientific Computing, Cambridge University Press, New York

Priest, E. R., 1982, Solar magneto-hydrodynamics, Kluwer Academic Publishers, Dordrecht

Priest, E. R., Forbes, T., 2000, Magnetic Reconnection, Cambridge University Press

Puschmann, K. G., Ruiz Cobo, B., Martínez Pillet, V., 2010, A Geometrical Height Scale for Sunspot Penumbrae, ApJ, 720, 1417-1431

Rachkovsky, D. N., 1962, Magnetic rotation effects in spectral lines, Izv. Krymsk. Astrofiz Obs., 28, 259

Rees, D. E., Semel, M. D., 1979, Line formation in an unresolved magnetic element - A test of the centre of gravity method, A\&A, 74, 1-5

Rees, D. E., Murphy, G. A., Durrant, C. J., 1989, Stokes Profile Analysis and Vector Magnetic Fields. II Formal Numerical Solutions of the Stokes Transfer equations, ApJ, 339,1093

Rempel, M., 2005, Solar differential rotation and meridional flow: the role of a subadiabatic tachocline for the Taylor-Proudman balance, ApJ, 622, 1320-1332

Rempel, M., 2011, Penumbral Fine Structure and Driving Mechanisms of Large-scale Flows in Simulated Sunspots, ApJ, 729, 5

Rempel, M., 2012a, High-latitude Solar Torsional Oscillations during Phases of Changing Magnetic Cycle Amplitude, ApJ, 750, 62

Rempel, M., 2012b, Numerical Sunspot Models: Robustness of Photospheric Velocity and Magnetic Field Structure, ApJ, 62, 21

Rempel, M., 2014, Numerical Simulations of Quiet Sun Magnetism: On the Contribution from a Small-scale Dynamo, ApJ, 789, 132

Rempel, M., 2015, Numerical simulations of sunspot decay: On the penumbra-Evershed flow-Moat flow connection, ApJ, 814, 125

Rempel, M., 2017, Extension of the MURaM radiative MHD code for coronal simulations, ApJ, 834, 10

Rempel, M., Schlichenmaier, R., 2011, Sunspot Modeling: From Simplified Models to Radiative MHD Simulations, Living Rev. Solar Phys., 8, 3 
Rempel, M., Schüssler, M., Cameron, R. H., Knölker, M., 2009a, Penumbral Structure and Outflows in Simulated Sunspots, Science, 325, 171

Rempel, M., Schüssler, M., Knölker, M., 2009b, Radiative Magnetohydrodynamic Simulation of Sunspot Structure, ApJ, 691, 640-649

Rezaei, R., Bello González, N., Schlichenmaier, R., 2012, The formation of sunspot penumbra. Magnetic field properties, A\&A, 537, A19

Richardson, W. H., 1972, Bayesian-Based Iterative Method of Image Restoration, JOSA, 62,55

Riethmüller, T. L., 2013, Investigations of small-scale magnetic features on the solar surface, Ph.D. thesis, Technische Universität Braunschweig, Germany, Germany

Riethmüller, T. L., Solanki, S. K., Lagg, A., 2008, Stratification of Sunspot Umbral Dots from Inversion of Stokes Profiles Recorded by Hinode, ApJ, 678, L157

Riethmüller, T. L., Solanki, S. K., Barthol, P., Gandorfer, A., Gizon, L., Hirzberger, J., van Noort, M., Rodríguez, J. B., Iniesta, J. C. D. T., Suárez, D. O., Schmidt, W., Pillet, V. M., Knölker, M., 2017, A new MHD-assisted Stokes Inversion Technique, ApJS, $229,16 \mathrm{R}$

Rimmele, T., 2008, ApJ, 674, 684

Rimmele, T. R., 1995, Sun center observations of the Evershed effect, ApJ, 445, 511

Rogers, F. J., 1994, IAU Colloq. 147: The Equation of State in Astrophysics, 147, 16

Rogers, F. J., Swenson, F. J., Iglesias, C. A., 1996, OPAL Equation-of-State Tables for Astrophysical Applications, ApJ, 456, 902

Romano, P., Frasca, D., Guglielmino, S. L., Ermolli, I., Tritschler, A., Reardon, K. P., Zuccarello, F., 2013, Velocity and Magnetic Field Distribution in a Forming Penumbra, ApJL, 771, L3

Romano, P., Guglielmino, S. L., Cristaldi, A., Ermolli, I., Falco, M., Zuccarello, F., 2014, Evolution of the Magnetic Field Inclination in a Forming Penumbra, ApJ, 784, 10

Rouppe van der Voort, L. H., Löfdahl, M. G., Kiselman, D., Scharmer, G. B., 2004, Penumbral structure at 0.1 arcsec resolution. I. General appearance and power spectra, A\&A, 414, 717

Rucklidge, A. M., Schmidt, H. U., Weiss, N. O., 1995, The abrupt development of penumbrae in sunspots, MNRAS, 273, 941

Rüedi, I., Solanki, S. K., Livingston, W., 1995, Infrared lines as probes of solar magnetic features. XI. Structure of a sunspot umbra with a light bridge, A\&A, 302, 550

Ruiz Cobo, B., 2007, Inversion techniques: From observations to atmospheres, in Modern Solar Facilities-Advanced Solar Science, (Eds.) F. Kneer, K. G. Puschmann, A. D. Wittmann, pp. 287-296 
Ruiz Cobo, B., Bellot Rubio, L. R., 2008, Heat transfer in sunspot penumbrae. Origin of dark-cored penumbral filaments, A\&A, 488, 749

Ruiz Cobo, B., del Toro Iniesta, J. C., 1992, Inversion of Stokes profiles, ApJ, 298, 375385

Sabatier, P. C., 2000, Journal of Mathem. Phys., 41, 4082

Sánchez Almeida, J., Lites, B. W., 1992, Observation and interpretation of the asymmetric Stokes Q, U, and V line profiles in sunspots, ApJ, 398, 359

Schad, T. A., Penn, M. J., 2010, Structural Invariance of Sunspot Umbrae over the Solar Cycle: 1993 - 2004, Sol. Phys., 262, 19-33

Scharmer, G. B., 2009, Recent Evidence for Convection in Sunspot Penumbrae, Space Sci. Rev., 144, 229

Scharmer, G. B., Spruit, H. C., 2006, Magnetostatic penumbra models with field-free gaps, A\&A, 460, 605-615

Scharmer, G. B., Gudiksen, B. V., Kiselman, D., Löfdahl, M. G., Rouppe van der Voort, L. H. M., 2002, Dark cores in sunspot penumbral filaments, Nature, 420, 151

Scharmer, G. B., Nordlund, A., Heinemann, T., 2008, Convection and the Origin of Evershed Flows in Sunspot Penumbrae, ApJL, 677, L149

Schlichenmaier, R., 2002, Penumbral fine structure: Theoretical understanding, AN, 323, 303

Schlichenmaier, R., 2009, Sunspots: From Small-Scale Inhomogeneities Towards a Global Theory, Space Sci. Rev., 144, 213

Schlichenmaier, R., Collados, M., 2002, Spectropolarimetry in a sunspot penumbra. Spatial dependence of Stokes asymmetries in Fe I 1564.8 nm, A\&A, 381, 668-682

Schlichenmaier, R., Schmidt, W., 2000, Flow geometry in a sunspot penumbra, A\&A, 358,1122

Schlichenmaier, R., Solanki, S. K., 2003, On the heat transport in a sunspot penumbra, A\&A, 411, 257-262

Schlichenmaier, R., Jahn, K., Schmidt, H. U., 1998a, A Dynamical Model for the Penumbral Fine Structure and the Evershed Effect in Sunspots, ApJ, 493, L121

Schlichenmaier, R., Jahn, K., Schmidt, H. U., 1998b, Magnetic flux tubes evolving in sunspots. A model for the penumbral fine structure and the Evershed flow, A\&A, 337, 897

Schlichenmaier, R., Bruls, J. H. M. J., Schüssler, M., 1999, Radiative cooling of a hot flux tube in the solar photosphere, A\&A, 349, 961-973 
Schlichenmaier, R., Bello González, N., Rezaei, R., Waldmann, T. A., 2010a, AN, 331, 563

Schlichenmaier, R., Bello González, N., Rezaei, R., Waldmann, T. A., 2010b, The formation of a sunspot penumbra, A\&A, 512, L1

Schlichenmaier, R., Rezaei, R., Bello González, N., 2012, On the Formation of Penumbrae as Observed with the German VTT SOHO/MDI, and SDO/HMI, in 4th Hinode Science Meeting: Unsolved Problems and Recent Insights, ASP Conference series, vol. 455 , p. 61

Schmidt, W., Hofmann, A., Balthasar, H., Tarbell, T. D., Frank, Z. A., 1992, Polarimetry and spectroscopy of a simple sunspot. I - On the magnetic field of a sunspot penumbra, A\&A, 264, L27

Schrijver, C. J., Zwaan, C., 2000, Solar and Stellar Magnetic Activity, Cambridge University Press. Cambridge astrophysics series, New York

Schröter, E. H., 1965, Der Evershed-Effekt in einem inaktiven symmetrischen Einzelfleck, ZAp, 62, 228

Schüssler, M., Vögler, A., 2006, Magnetoconvection in a sunspot umbra, ApJ, 641, L73

Schwarz, G. E., 1978, Ann. Stat., 6, 461

Semel, M., 1967, Contribution a létude des champs magnétiques dans les régions actives solaires, AnAp, 30, 513-513

Semel, M., 1970, A Proposal for a Solar Magnetograph, A\&A, 5, 330-332

Shenstone, A. G., Blair, H. A., 1929, Phil. Mag., 8, 765

Shimizu, T., Ichimoto, K., Suematsy, Y., 2012, Precursor of Sunspot Penumbral Formation Discovered with Hinode Solar Optical Telescope Observations, ApJL, 747, L18

Shurcliff, W. A., 1962, Polarized Light: Production and Use, Harvard University Press, Cambridge

Simon, G. W., Weiss, N. O., 1970, On the Magnetic Field in Pores, Sol. Phys., 13, 85-103

Siu-Tapia, A. L., Lagg, A., Solanki, S. K., van Noort, M., Jǔrcák, J., 2017, Normal and counter Evershed flows in the photospheric penumbra of a sunspot. SPINOR 2D inversions of Hinode-SOT/SP observations, A\&A, 607, A36

Siu-Tapia, A. L., Rempel, M., Lagg, A., Solanki, S. K., 2018, Evershed and CounterEvershed Flows in Sunspot MHD Simulations, ApJ, 852, 66

Skumanich, A., Lites, B. W., 1987, Stokes profile analysis and vector magnetic fields. I Inversion of photospheric lines, ApJ, 322, 473 
Skumanich, A., Lites, B. W., Martínez Pillet, V., 1994, Vector spectropolarimetry with the Advanced Stokes Polarimeter (ASP) for quantitative solar magnetometry, Solar Surface Magnetism, p. 99

Sobotka, M., Bonet, J. A., Vázquez, M., Hanslmeier, A., 1995, On the Dynamics of Bright Features in Sunspot Umbrae, ApJ, 447, L133

Socas-Navarro, H., 2001, Stokes Inversion Techniques: Recent Achievements and Future Horizons, ASP Conference Series, 236, 487-501

Socas-Navarro, H., 2005, Are electric currents heating the magnetic chromosphere?, ApJL, 633, L57-L60

Socas-Navarro, H., Martínez Pillet, V., Sobotka, M., Vázquez, M., 2004, The Thermal and Magnetic Structure of Umbral Dots from the Inversion of High-Resolution Full Stokes Observations, ApJ, 614, 448-456

Solanki, S. K., 1987, The Photospheric Layers of Solar Magnetic Fluxtubes, Ph.D. thesis, Institute of Astronomy, ETH Zürich, Switzerland, no. 8309

Solanki, S. K., 1993, Small scale Solar Magnetic Fields - an Overview, Space Sci. Rev., $63,1-188$

Solanki, S. K., 1997, Empirical Modelling and Thermal Structure of Sunspots, 1st Advances in Solar Physics Euroconference. Advances in Physics of Sunspots, 118, 178

Solanki, S. K., 2003, Sunspots: An overview, A\&A Rv, 11, 153

Solanki, S. K., Montavon, C. A. P., 1993, Uncombed fields as the source of the broad-band circular polarization of sunspots, A\&A, 275, 283-292

Solanki, S. K., Stenflo, J. O., 1984, Properties of solar magnetic fluxtubes as revealed by Fe I lines, A\&A, 140, 185

Solanki, S. K., Keller, C., Stenflo, J. O., 1987, Properties of solar magnetic fluxtubes from only two spectral lines, A\&A, 188, 183

Solanki, S. K., Rüedi, I., Livingston, W., 1992a, Infrared lines as probes of solar magnetic features. II - Diagnostic capabilities of Fe I 15648.5 A and 15652.9 A, A\&A, 263, 312

Solanki, S. K., Rüedi, I., Livingston, W., 1992b, Infrared lines as probes of solar magnetic features. V - The magnetic structure of a simple sunspot and its canopy, A\&A, 263, 339

Solanki, S. K., Walther, U., Livingston, W., 1993, Infrared lines as probes of solar magnetic features. VI. The thermal-magnetic relation and Wilson depression of a simple sunspot, A\&A, 277, 639-647

Solanki, S. K., Montavon, C. A. P., Livingston, W., 1994, Infrared lines as probes of solar magnetic features. 7: On the nature of the Evershed effect in sunspots, A\&A, 283, 221-231 
Solanki, S. K., Lagg, A., Woch, J., Krupp, N., Collados, M., 2003, Three-dimensional magnetic field topology in a region of solar coronal heating, Nature, 425, 692-695

Spiegel, E. A., Weiss, N. O., 1980, Magnetic activity and variations in solar luminosity, Nature, 287, 616

Spiegel, E. A., Zahn, J. P., 1992, The solar tachocline, A\&A, 265, 106

Spruit, H. C., 1979, Convective collapse of flux tubes, Sol. Phys., 61, 363-378

Spruit, H. C., Scharmer, G. B., 2006, Fine structure, magnetic field and heating of sunspot penumbrae, A\&A, 447, 343

St. John, C. E., 1913, Radial Motion in Sun-Spots, ApJ, 37, 322

Stenflo, J. O., 1973, Magnetic-Field Structure of the Photospheric Network, Sol. Phys., 32,41

Stenflo, J. O., 1993, Solar magnetic fields, in Proc. Int. Conf., p. 301, Cambridge University Press, Feidburg, Germany

Stenflo, J. O., Harvey, J. W., 1985, Dependence of the properties of magnetic fluxtubes on area factor or amount of flux, Sol. Phys., 95, 99

Stift, M. J., Leone, F., Landi Degl'Innocenti, E., 2008, MNRAS, 385, 1813-1819

Stix, M., 2002, The Sun: An Introduction, Springer, 79104 Freiburg, Germany

Stokes, G. G., 1852, Phil. Trans. R. Soc. Lond., 142, 463-562

Strous, L. H., 1994, Dynamics of Solar Active Regions: Patterns in Magnetic-Flux Emergence, Ph.D. thesis, University of Utrecht, Netherlands

Suematsu, Y., Tsuneta, S., Ichimoto, K., Shimizu, T., Otsubo, M., Katsukawa, Y., Nakagiri, M., Noguchi, M., Tamura, T., Kato, Y., Hara, H., Kubo, M., Mikami, I., Saito, H., Matsushita, T., Kawaguchi, N., Nakaoji, T., Nagae, K., Shimada, S., Takeyama, N., Yamamuro, T., 2008, The Solar Optical Telescope of Solar-B ( Hinode): The Optical Telescope Assembly, Sol. Phys., 249, 197-220

Thomas, J. H., 1994, Solar Surface Magnetism

Thomas, J. H., Montesinos, B., 1993, A siphon-flow model of the photospheric Evershed flow in a sunspot, ApJ, 407, 398

Thomas, J. H., Weiss, N. O., 1992, Sunspots: Theory and observations, in Proceedings of the NATO Advanced Research Workshop on The Theory of Sunspots, vol. 375, NATO Science Series C, Cambridge, U. K.

Thomas, J. H., Weiss, N. O., 2004, Fine structure of sunspots, ARA\&A, 42, 517-548

Thomas, J. H., Weiss, N. O., 2008, Sunspots and Starspots, Cambridge University Press, Cambridge 
Thomas, J. H., Weiss, N. O., Tobias, S. M., Brummell, N. H., 2002, Downward pumping of magnetic flux as the cause of filamentary structures in sunspot penumbrae, Nature, 420,390

Thomas, J. H., Weiss, N. O., Tobias, S. M., Brummell, N. H., 2006, On the fine structure of magnetic fields in sunspot penumbrae, A\&A, 452, 1089-1090

Thompson, M. J., Christensen-Dalsgaard, J., Miesch, M. S., Toomre, J., 2003, The Internal Rotation of the Sun, ARA\&A, 41, 599

Title, A. M., Frank, Z. A., Shine, R. A., Tabell, T. D., Topka, K. P., Scharmer, G., Schmidt, W., 1993, On the magnetic and velocity field geometry of simple sunspots, ApJ, 403, 780

Tiwari, S. K., van Noort, M., Lagg, A., Solanki, S. K., 2013, Structure of sunspot penumbral filaments: a remarkable uniformity of properties, A\&A, 557, A25

Tiwari, S. K., van Noort, M., Solanki, S. K., Lagg, A., 2015, Depth-dependent global properties of a sunspot observed by Hinode using the Solar Optical Telescope/Spectropolarimeter, A\&A, 583, A119

Tkaczuk, R., Rieutord, M., Meunier, N., Roudier, T., 2007, Tracking granules on the Sun's surface and reconstructing velocity fields., A\&A, 471, 695-703

Tomczyk, S., Elmore, D. F., Lites, B. W., Dunn, R. B., Skumanich, A., Schuenke, J. A., Streander, K. V., Leach, T. W., Chambellan, C. W., Lacey, L. B., 1992, The Advanced Stokes Polarimeter: A New Instrument for Solar Magnetic Field Research, American Astronomical Society Meeting, 180, 5108

Tritschler, A., 2009, Structure and Dynamics of Sunspots (Keynote), in The Second Hinode Science Meeting: Beyond Discovery-Toward Understanding, ASP Conference Series, (Eds.) B. Lites, M. Cheung, T. Magara, J. Mariska, K. Reeves, vol. 415, p. 339

Tsiropoula, G., 2000, Physical parameters and flows along chromospheric penumbral fibrils, A\&A, 357, 735-742

Tsuneta, S., Suematsu, Y., Ichimoto, K., Shimizu, T., Otsubo, M., Shimizu, T., Suematsu, Y., Nakagiri, M., Noguchi, M., Tarbell, T., Title, A., Rosenberg, R. S. W., Hoffmann, C., Jurcevich, B., Kushner, G., Levay, M., Lites, B., Elmore, D., Matsushita, T., Kawaguchi, N., Saito, H., Mikami, I., Hill, L. D., Owens, J. K., 2008, The Solar Optical Telescope for the Hinode Mission: An Overview, Sol. Phys., 249, 167

Tziotzio, K., Heinzel, P., Mein, P., Mein, N., 2001, Non-LTE inversion of chromospheric Ca cloud-like features, A\&A, 366, 686-689

Unno, W., 1956, Line Formation of a Normal Zeeman Triplet, Publ. Astron. Soc. Japan, 8,108

van Noort, M., 2012, Spatially coupled inversion of spectro-polarimetric image data I. Method and first results, A\&A, 548, A5 
van Noort, M., Lagg, A., Tiwari, S. K., Solanki, S. K., 2013, Peripheral downflows in sunspot penumbrae, A\&A, 557, A24

Venkatakrishnan, P., 1986, Inhibition of convective collapse of solar magnetic flux tubes by radiative diffusion, Nature, 322, 156

Vernazza, J. E., Avrett, E. H., Loeser, R., 1976, Structure of the solar chromosphere. II. The underlying photosphere and temperature-minimum region, ApJ, 30, 1-60

Vögler, A., 2003, Ph.D. thesis, University of Göttingen, Germany, Germany, http://www.solar-system-school.de/alumni/voegler.pdf

Vögler, A., 2004, Effects of non-grey radiative transfer on 3D simulations of solar magneto-convection, A\&A, 421, 755

Vögler, A., Schüssler, M., 2007, A solar surface dynamo, A\&A, 465, L43

Vögler, A., Bruls, J. H. M. J., Schüssler, M., 2004, Approximations for non-grey radiative transfer in numerical simulations of the solar photosphere, A\&A, 421, 741

Vögler, A., Shelyag, S., Schüssler, M., Cattaneo, F., Emonet, T., Linde, T., 2005, Simulations of magneto-convection in the solar photosphere. Equations, methods, and results of the MURaM code, A\&A, 429, 335

Vrabec, D., 1971, Magnetic Fields Spectroheliograms from the San Fernando Observatory, Solar Magnetic Fields, IAU Symp., 43, 329

Vrabec, D., 1974, Chromospheric Fine Structure, IAU Symp., 56, 201

Walker, L. M., 2006, The random walk of radiation from the sun

Wedemeyer-Böhm, S., Lagg, A., Nordlund, A., 2009, Coupling from the Photosphere to the Chromosphere and the Corona, Space Sci. Rev., 144, 317-350

Weiss, N. O., 1966, The expulsion of magnetic flux by eddies, Proc. Roy Soc. London A, 293,310

Weiss, N. O., Thomas, J. H., Brummell, N. H., Tobias, S. M., 2004, The Origin of Penumbral Structure in Sunspots: Downward Pumping of Magnetic Flux, ApJ, 600, 1073

Westendorp Plaza, C., del Toro Iniesta, J. C., Ruiz Cobo, B., Martínez Pillet, V., Lites, B. W., Skumanich, A., 1997, Evidence for a downward mass flux in the penumbral region of a sunspot, Nature, 389, 47-49

Westendorp Plaza, C., del Toro Iniesta, J. C., Ruiz Cobo, B., Martínez Pillet, V., 2001a, Optical Tomography of a Sunspot. III. Velocity Stratification and the Evershed Effect, ApJ, 547, 1148-1158

Westendorp Plaza, C., del Toro Iniesta, J. C., Ruiz Cobo, B., Martínez Pillet, V., Lites, B. W., Skumanich, A., 2001b, Optical Tomography of a Sunspot. II. Vector Magnetic Field and Temperature Stratification, ApJ, 547, 1130 
Wiehr, E., 1978, A Unique Magnetic Field Range for Nonspot Solar Magnetic Regions, A\&A, 69, 279

Wiehr, E., 1995, The origin of the Evershed asymmetry, A\&A, 289, L17

Wittman, A., 1974, Computation and observation of Zeeman multiplet polarization in Frauenhofer lines, Sol. Phys., 35, 11

Yang, G., Xu, Y., Wang, H., Denker, C., 2003, High Spatial Resolution Observations of Pores and the Formation of a Rudimentary Penumbra, ApJ, 597, 1190

Zakharov, V., Hirzberger, J., Riethmüller, T. L., Solanki, S. K., Kobel, P., 2008, Evidence of convective rolls in a sunspot penumbra, A\&A, 488, L17

Zeeman, P., 1897, On the Influence of Magnetism on the Nature of the Light Emitted by a Substance, ApJ, 5, 332

Zhou, G., Wang, J., Jin, C., 2013, Solar Intranetwork Magnetic Elements: Evolution and Lifetime, Sol. Phys., 267, 63-73

Zuccarello, F., Guglielmino, S. L., Romano, P., 2014, Evolution and Dynamics of Orphan Penumbrae in the Solar Photosphere: Analysis from Multi-instrument Observations, ApJ, 787, 57

Zurbuchen, T. H., Richardson, I. G., 2006, In-Situ Solar Wind and Magnetic Field Signatures of Interplanetary Coronal Mass Ejections, Space Sci. Rev., 123, 31-34

Zwaan, C., 1985, The emergence of magnetic flux, Sol. Phys., 100, 397

Zwaan, C., 1987, Elements and patterns in the solar magnetic field, ARA\&A, 25, 83

Zwaan, C., 1992, The evolution of sunspots, In: Sunspots: Theory and observations; Proceedings of the NATO Advanced Research Workshop on the Theory of Sunspots, Cambridge, United Kingdom 




\section{Publications}

\section{Refereed publications}

- A. L. Siu-Tapia, M. Rempel, A. Lagg and S. K. Solanki, (2018). Evershed and Counter-Evershed Flows in Sunspot MHD Simulations, ApJ, 852, 66.

- A. Siu-Tapia, A. Lagg, S. K. Solanki, M. van Noort and J. Jurčák, (2017). Normal and counter Evershed flows in the photospheric penumbra of a sunspot. SPINOR $2 D$ inversions of Hinode-SOT/ SP observations, A\&A, 607, A36.

\section{Conference contributions}

- SOLARNET IV MEETING: The Physics of the Sun from the Interior to the Outer Atmosphere. Lanzarote, Spain. 16-20 January, 2017. Talk: Photospheric counter Evershed flows in the penumbra of sunspots.

- IAU Symposium 327: Fine Structure and Dynamics of the Solar Atmosphere. Cartagena de Indias, Colombia. 09-14 October, 2016. Talk: Anomalous counterEvershed flows: HINODE observations and MHD simulations.

- Hinode 9 - International Science Meeting. Belfast, Northern Ireland. 14-18 September, 2015. ePoster: Anomalous plasma inflow in the penumbra of a sunspot.

- Sunspot formation: theory, simulations and observations. Stockholm, Sweden. 0913 March, 2015. Poster: Anomalous reversed Evershed flow in a sunspot penumbra. 



\section{Acknowledgements}

First of all, I am deeply grateful to my supervisor, Dr. Andreas Lagg, for his full support, excellent guidance, constant encouragement, and enjoyable discussions throughout my $\mathrm{PhD}$ research. Also, I thank him for being available at all times to address the unforeseen problems and for giving me the freedom to develop my work according to my preferences. His deep understanding in solar physics combined with his cheerful and easy-going personality represent to me the essential aspects that make him an excellent supervisor.

I also thank my supervisor Prof. Dr. Sami K. Solanki for constantly taking the time in revising the progress of my work and for giving me the opportunity to participate at different conferences and meetings to present my work. I thank him for reading and improving this thesis and the manuscripts that resulted from this research, which were all largely benefited from his excellent scientific writing as well as from his broad knowledge in the field.

I would like to thank the members of my thesis advisory committee: Prof. Dr. Ansgar Reiners, Prof. Dr. Sami Solanki, and Dr. Andreas Lagg, as well as the additional members of my jury: Prof. Dr. Wolfrang Kollatschny, Dr. Jorrit Leenaarts, Dr. Maarit Käpylä, Prof. Dr. Hardi Peter, and Prof. Dr. Stefan Dreizler for taking the time in revising this work.

My sincere gratitude to Dr. Matthias Rempel, who largely contributed to this work and was always open to collaborate. He gladly provided me with his simulation data when requested, and invited me to visit him at HAO Boulder to work together during a month. I want to acknowledge my debt to him, given that his deep understanding in solar physics as well as his expertise in magnetohydrodynamics were both indispensable help for carrying out this work.

During the three years of my $\mathrm{PhD}$, I could benefit from the expertise of several people within the solar physics community. Special thanks to Dr. Michiel van Noort, Dr. Nazaret Bello González, Dr. Rolf Schlichenmaier and Dr. Jan Jurčák for all the fruitful discussions and suggestions regarding the interpretation of the inversion results, and for shedding new light on the possible origin of CEF. Thank you all for your insightful comments and directions that substantially contributed to gather new information on a poorly understood aspect of sunspot penumbrae.

I would also like to thank Dr. Sonja Schuh for all her help, and to Ines, Claudia and Sibylla for their support in the administrative matters.

This work was carried out in the frame of the International Max Planck Research School (IMPRS) for Solar System Science at the Max Planck Institute for Solar System Research (MPS). It was supported by the Max Planck Society and by BECAS CONACyT AL EXTRANJERO 2014.

In the following I would like to thank other people: 
Thanks to Kinga, Iker, Mariangela, Francisco, Ivan, Michel, Ricardo and Iulia for your friendship and good company.

Muchas gracias a mis padres, hermanas, abuelita Mede, y tía Nery por su constante e incondicional apoyo. A pesar de los miles de kilómetros de distancia que nos separaron durante estos tres años, sus cálidas porras siempre me animaron y consolaron en éste lugar que se vuelve tan frío y gris durante invierno.

A osito, ositito, pingüinito, monita, perrito, poussin, Benji, mapachito, renito, y dragoncito. Gracias por endulzar mi vida.

Enfin et surtout, merci beaucoup Benjamin, pour ta compagnie et ton soutien sincère dès le premier jour où j'ai marché sur ces terres. Sans cela, sans ton affection inégalée, cette expérience aurait été très difficile. Tu es la meilleure chose que je retire de cette période. 


\title{
Curriculum vitae
}

\author{
Name: Azaymi Litzi \\ Last name: Siu Tapia \\ Date of birth: 08 September, 1988 \\ Nationality: Mexican \\ Education:
}

- Bachelor of Sciences (Physics)

Faculty of Sciences, Universidad Nacional Autónoma de México

Mexico City, Mexico.

- Master of Sciences (Space Physics)

Instituto de Geofísica, Universidad Nacional Autónoma de México

Mexico City, Mexico.

- PhD (Solar Physics)

International Max Planck Research School at University of Göttingen

Göttingen, Germany.

\section{Publications:}

- A. L. Siu-Tapia, M. Rempel, A. Lagg and S. K. Solanki, (2018). Evershed and Counter-Evershed Flows in Sunspot MHD Simulations, ApJ, 852, 66.

- A. Siu-Tapia, A. Lagg, S. K. Solanki, M. van Noort and J. Jurčák, (2017). Normal and counter Evershed flows in the photospheric penumbra of a sunspot. SPINOR $2 D$ inversions of Hinode-SOT/SP observations, A\&A, 607, A36.

- A. Siu-Tapia, X. Blanco-Cano, P. Kajdic, E. Aguilar-Rodríguez, C. T. Russell, L. K. Jian, L. K. and J. G. Luhmann, (2015). Low-frequency waves within isolated magnetic clouds and complex structures: STEREO observations, JGR, 120, 23632381. 